\title{
Coupling of Trifluoroacetaldehyde $N$-Triftosylhydrazone with Organoboronic Acids for the Synthesis of gem-Difluoroalkenes
}

Yu Ma, ${ }^{\dagger, \dagger}$ Bhoomireddy Rajendra Prasad Reddy ${ }^{\dagger}$ and Xihe $\mathrm{Bi}^{*}, \dagger, \dot{\dagger}$

$\dagger$ Jilin Province Key Laboratory of Organic Functional Molecular Design \& Synthesis, Department of Chemistry, Northeast Normal University, Changchun 130024, China.

\$ State Key Laboratory of Elemento-Organic Chemistry, Nankai University, Tianjin 300071, China.

Corresponding Authors:

*bixh507@nenu.edu.cn

\section{Table of contents}

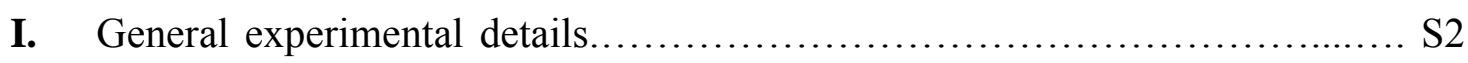

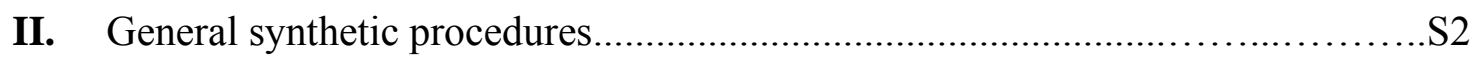

III. Characterization data for the products................................. 7

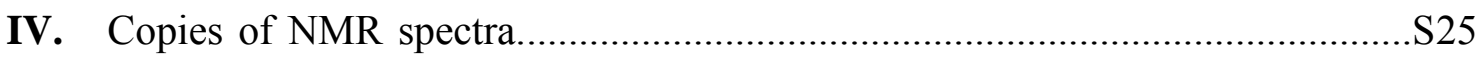




\section{General experimental details}

All reagents and solvents were purchased from commercial sources and used without purification unless otherwise stated. The products were purified by column chromatography over silica gel (200400 size). The ${ }^{1} \mathrm{H},{ }^{13} \mathrm{C}$ and ${ }^{19} \mathrm{~F}-\mathrm{NMR}$ were recorded in $\mathrm{CDCl}_{3}$ using Bruker AV-600 (600 MHz for $1 \mathrm{H}, 150 \mathrm{MHz}$ for ${ }^{13} \mathrm{C}$ and $564 \mathrm{MHz}$ for ${ }^{19} \mathrm{~F}$ ) and Agilent (Varian)-500 (500 MHz for ${ }^{1} \mathrm{H}, 125 \mathrm{MHz}$ for ${ }^{13} \mathrm{C}$ and $470 \mathrm{MHz}$ for ${ }^{19} \mathrm{~F}$ ) spectrometers using TMS as internal standard. Coupling constants are given in hertz. The following abbreviations are used: s, singlet; d, doublet; t, triplet; q, quadruplet, quint., quintet, m, multiplet, dd, doublet of doublet, tt, triplet of triplet, td, triplet of doublet. Mass spectra were recorded on TSQ 8000 Evo by using EI method. High resolution mass spectra (HRMS) were recorded on Bruck microTof by using ESI method.

\section{General synthetic procedures}

General procedure A: Synthesis of alkyl gem-difluoroalkenes

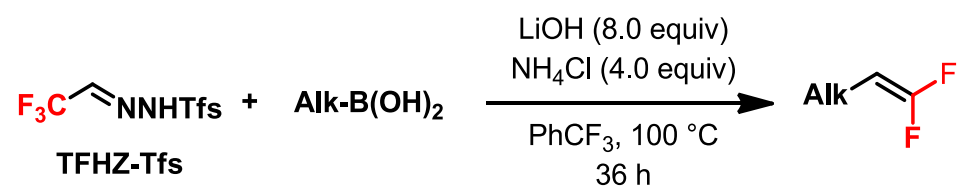

A screw capped reaction vial was charged with trifluoroacetaldehyde $N$-tfsylhydrazone (TFHZ-Tfs) (200 mg, $0.625 \mathrm{mmol}$ ), alkylboronic acid (0.25 mmol), $\mathrm{LiOH}$ (48.0 mg, $2.0 \mathrm{mmol})$, and $\mathrm{NH}_{4} \mathrm{Cl}(53.5$ $\mathrm{mg}, 1.0 \mathrm{mmol}$ ). Then the reaction vial was repeatedly purged with argon, and finally added the 5.0 $\mathrm{mL}$ of $\mathrm{PhCF}_{3}$ by syringe. The mixture was stirred in an oil bath at $100{ }^{\circ} \mathrm{C}$ for $36 \mathrm{~h}$. After the reaction mixture had cooled down to room temperature, filtered through a short silica bed eluting with DCM. The solvent was evaporated under reduced pressure to leave a crude product, which was purified by flash column chromatography on silica gel eluting with petroleum ether (PE) to afford the alkyl gem-difluoroalkene.

General procedure B: Synthesis of aryl gem-difluoroalkenes

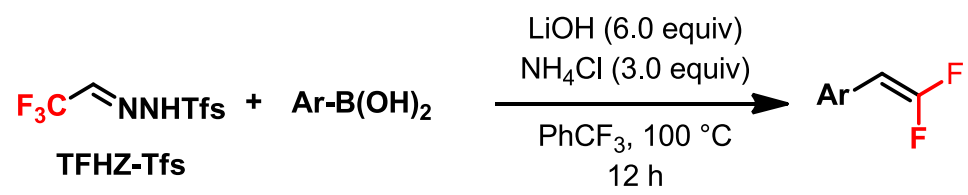

A screw capped reaction vial was charged with TFHZ-Tfs ( $240 \mathrm{mg}, 0.75 \mathrm{mmol})$, arylboronic acid (0.3 mmol), $\mathrm{LiOH}(43.2 \mathrm{mg}, 1.8 \mathrm{mmol})$ and $\mathrm{NH}_{4} \mathrm{Cl}(48.1 \mathrm{mg}, 0.9 \mathrm{mmol})$. Then the reaction vial was 
repeatedly purged with argon, and finally added the $4.5 \mathrm{~mL}$ of $\mathrm{PhCF}_{3}$ by syringe. The mixture was stirred in an oil bath at $100{ }^{\circ} \mathrm{C}$ for $12 \mathrm{~h}$. After the reaction mixture had cooled down to room temperature, filtered through a short silica bed eluting with DCM. The solvent was evaporated under reduced pressure to leave a crude product, which was purified by flash column chromatography on silica gel eluting with PE to afford the aryl gem-difluoroalkene.

\section{General procedure C: Gram-scale synthesis of 20}

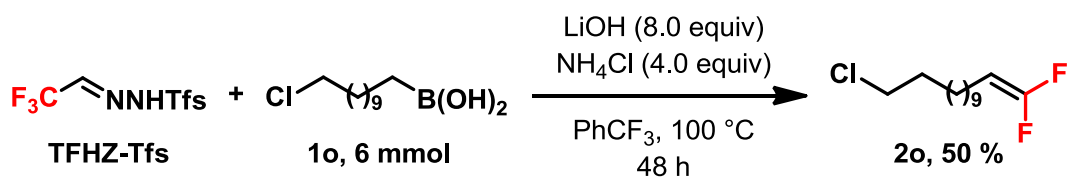

A screw capped reaction vial was charged with trifluoroacetaldehyde $N$-tfsylhydrazone (TFHZ-Tfs) (4.8 g, $15.0 \mathrm{mmol})$, alkylboronic acid (6.0 mmol), $\mathrm{LiOH}(1.15 \mathrm{mg}, 48.0 \mathrm{mmol})$, and $\mathrm{NH}_{4} \mathrm{Cl}(1.28$ $\mathrm{mg}, 24.0 \mathrm{mmol})$. Then the reaction vial was repeatedly purged with argon, and finally added the 100 $\mathrm{mL}$ of $\mathrm{PhCF}_{3}$ by syringe. The mixture was stirred in an oil bath at $100{ }^{\circ} \mathrm{C}$ for $48 \mathrm{~h}$. After the reaction mixture had cooled down to room temperature, filtered through a short silica bed eluting with DCM. The solvent was evaporated under reduced pressure to leave a crude product, which was purified by flash column chromatography on silica gel eluting with PE to afford the alkyl gem-difluoroalkene.

General procedure D: Synthesis of TFHZ-Tfs

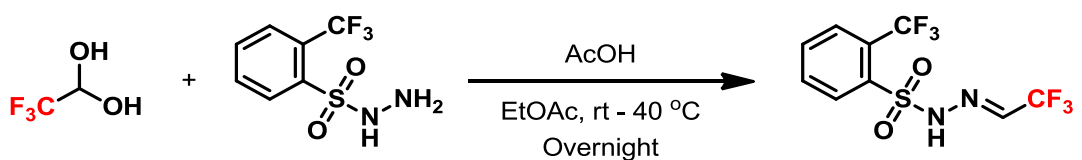

To a solution of trifluoroacetaldehyde monohydrate $(3.8 \mathrm{~g}, \quad 30.0 \mathrm{mmol}), \quad o-$ trifluoromethylbenzenesulfonyl hydrazide $(4.8 \mathrm{~g}, 20.0 \mathrm{mmol})$ in EtOAc $(50.0 \mathrm{~mL})$ was added $\mathrm{CH}_{3} \mathrm{COOH}(1.0 \mathrm{~mL}, 1.8 \mathrm{mmol})$ at $0{ }^{\circ} \mathrm{C}$ under nitrogen. Then the temperature was raised to room temperature and stirred for 80 mins. After that, the reaction was placed in a $40{ }^{\circ} \mathrm{C}$ oil bath and stirred overnight at this temperature, and monitored by TLC (PE:EtOAc $=2: 1)$. After completion of the reaction, solvent was evaporated under reduced pressure to leave a crude product, which was gently washed with PE/EtOAc (10:1) to afford the pure product as a white solid.

\section{General procedure E: Synthesis of alkylboronic acids 1e,1g-1s}




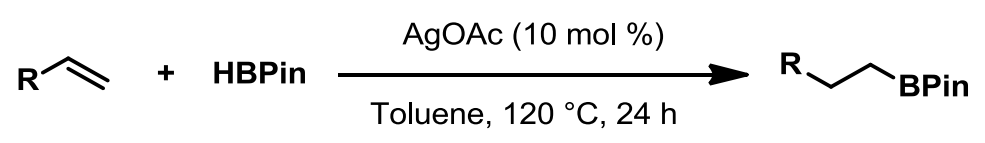

Prepared according to the slightly modified procedure of $\mathrm{Bi}$ et al. ${ }^{1}$ To a schlenk tube placed with AgOAc (170.0 mg, $0.1 \mathrm{mmol})$, alkenes $(10.0 \mathrm{mmol})$, and toluene $(20.0 \mathrm{~mL})$ was added HBpin (2.0 g, $15.0 \mathrm{mmol}$ ) drop wise by syringe over one minute under argon. The resulting mixture was stirred in an oil bath at $120^{\circ} \mathrm{C}$ for $24 \mathrm{~h}$. After cooling down to room temperature, the reaction mixture was diluted with $30.0 \mathrm{~mL}$ of EtOAc and filtered through a celite plug. The solvent was evaporated under reduced pressure to leave a crude product, which was purified by column chromatography on silica gel eluting with PE/EtOAc.

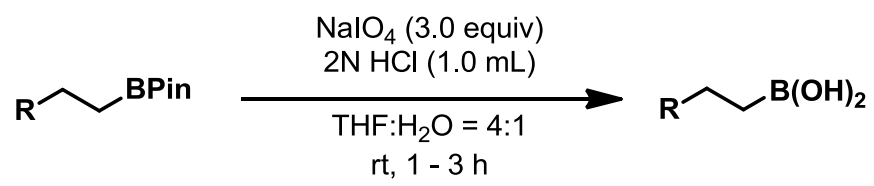

Prepared according to the slightly modified procedure of Li et al. ${ }^{2} n$-Alkylboronic esters ( $\left.3.0 \mathrm{mmol}\right)$ was dissolved in $25 \mathrm{~mL}$ mixed solvent of THF:water (4:1). To this solution, $\mathrm{NaIO}_{4}(1.9 \mathrm{~g}, 3.0$ equiv) was added and stirred for $5 \mathrm{~min}$. Then an aqueous solution of $\mathrm{HCl}(2.0 \mathrm{~N}, 1.0 \mathrm{~mL})$ was added and stirred until the $n$-alkylboronic esters were completely consumed as monitored by TLC. The reaction mixture was extracted with EtOAc. The combined organic layers were washed with brine, dried over anhydrous $\mathrm{Na}_{2} \mathrm{SO}_{4}$, filtered, and solvent was evaporated to leave the crude $n$-alkylboronic acids, which was recrystallized from PE/EtOAc to give the corresponding $n$-alkylboronic acid.

\section{General procedure F: Synthesis of alkenylboronic acids 1t-1v}

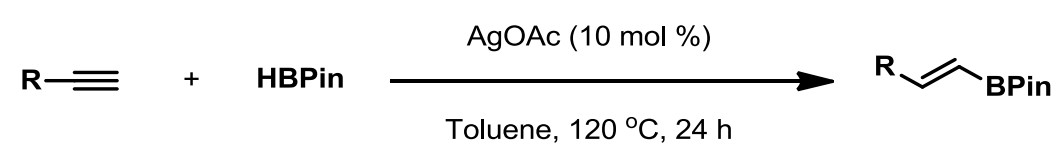

Prepared according to the slightly modified procedure of Bi et al. ${ }^{1}$ To a schlenk tube placed with AgOAc (170.0 mg, $0.1 \mathrm{mmol})$, alkynes (10.0 mmol), and toluene (20.0 mL) was added HBpin (2.0 g, $15.0 \mathrm{mmol}$ ) drop wise by syringe over one minute under argon. The resulting mixture was stirred in an oil bath at $120^{\circ} \mathrm{C}$ for $24 \mathrm{~h}$. After cooling down to room temperature, the reaction mixture was diluted with $30.0 \mathrm{~mL}$ of EtOAc and filtered through a celite plug. The solvent was evaporated under reduced pressure to leave a crude product, which was purified by column chromatography on silica 


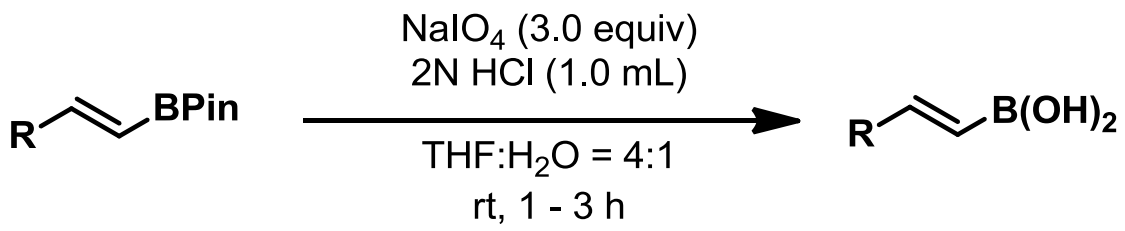

Prepared according to the slightly modified procedure of Li et al. ${ }^{2}$ Alkenylboronic esters ( $\left.3.0 \mathrm{mmol}\right)$ was dissolved in $25 \mathrm{~mL}$ mixed solvent of THF:water (4:1). To this solution, $\mathrm{NaIO}_{4}(1.9 \mathrm{~g}, 3.0$ equiv) was added and stirred for $5 \mathrm{~min}$. Then an aqueous solution of $\mathrm{HCl}(2.0 \mathrm{~N}, 1.0 \mathrm{~mL})$ was added and stirred until the $n$-alkenylboronic esters were completely consumed as monitored by TLC. The reaction mixture was extracted with EtOAc. The combined organic layers were washed with brine, dried over anhydrous $\mathrm{Na}_{2} \mathrm{SO}_{4}$, filtered, and solvent was evaporated to leave the crude $n$ alkenylboronic acids, which was recrystallized from PE/EtOAc to give the corresponding $n$ alkenylboronic acid.

\section{General procedure G: Synthesis of 5}

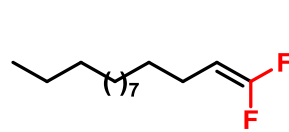

2c

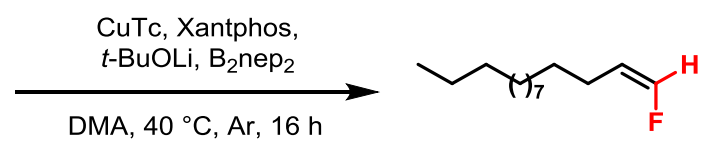

5, $72 \%$

A screw capped reaction vial was charged with CuTc (3.8 mg, 10 mol \%), Xantphos (11.6 mg, 10 mol \%), $\mathrm{B}_{2} \mathrm{nep}_{2}(135.5 \mathrm{mg}, 0.6 \mathrm{mmol}), 2 \mathrm{c}(46.4 \mathrm{mg}, 0.2 \mathrm{mmol})$, and $t$-BuOLi (48.0 mg, $\left.0.6 \mathrm{mmol}\right)$. Then the reaction vial was repeatedly purged with argon, and finally added the $1.0 \mathrm{~mL}$ of DMA by syringe. The mixture was stirred in an oil bath at $40{ }^{\circ} \mathrm{C}$ for $16 \mathrm{~h}$. After the reaction mixture had cooled down to room temperature, filtered through a short silica bed eluting with EtOAc. The solvent was evaporated under reduced pressure to leave a crude product, which was purified by flash column chromatography on silica gel eluting with PE to afford the product $\mathbf{5}$.

\section{General procedure H: Synthesis of 6}

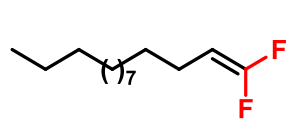

2c

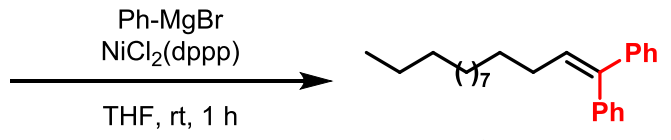

6, $65 \%$ 
A screw capped reaction vial was charged with $\mathrm{NiCl}_{2}(\mathrm{dppp})(10.8 \mathrm{mg}, 5 \mathrm{~mol} \%)$. Then the reaction vial was repeatedly purged with argon, and added $2 \mathbf{c}(69.6 \mathrm{mg}, 0.3 \mathrm{mmol}), 2.5 \mathrm{~mL}$ of THF and phenyl Grignard reagent in THF (1.5mmol, 1.0 M) (drop-wise) with syringe. The mixture was stirred at room temperature for $1 \mathrm{~h}$. After completion of the reaction quenched with saturated $\mathrm{NH}_{4} \mathrm{Cl}$ $(5.0 \mathrm{~mL})$ and extracted with EtOAc. The combined organic extracts were washed with water and brine, dried over anhydrous $\mathrm{Na}_{2} \mathrm{SO}_{4}$, filtered and solvent was evaporated under reduced pressure to leave a crude product, which was purified by flash column chromatography on silica gel eluting with PE/EtOAc (20:1) to afford the product 6.

\section{General procedure I: Synthesis of 7}

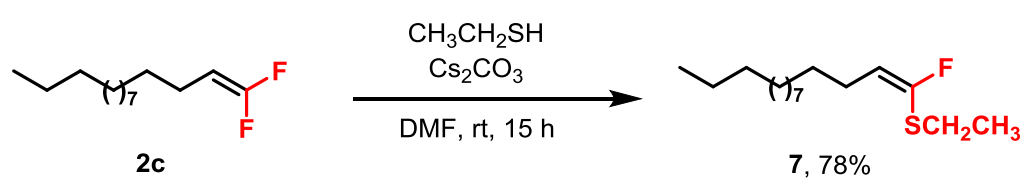

A screw capped reaction vial was charged with $\mathrm{Cs}_{2} \mathrm{CO}_{3}(35.0 \mathrm{mg}, 0.15 \mathrm{mmol})$. Then the reaction vial was repeatedly purged with argon, and added $2.0 \mathrm{~mL}$ of DMF, $2 \mathbf{c}(69.6 \mathrm{mg}, 0.3 \mathrm{mmol})$ and thioethanol (18.7 mg, $0.3 \mathrm{mmol})$ with syringe. The mixture was stirred at room temperature for 15 h. After completion of the reaction the reaction mixture was washed with EtOAc $(3 \times 10 \mathrm{~mL})$ and solvent was evaporated under reduced pressure to leave a crude product, which was purified by flash column chromatography on silica gel eluting with PE/EtOAc (20:1) to afford the product 7.

\section{General procedure J: Synthesis of 8}

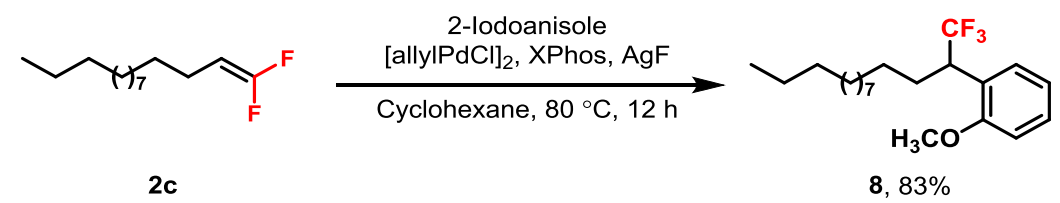

A screw capped reaction vial was charged with 2-iodoanisole $(35.0 \mathrm{mg}, 0.15 \mathrm{mmol})$, [allylPdCl$]_{2}$ (11.1 mg, $2.5 \mathrm{~mol} \%$ ), XPhos (6.7 mg, $10 \mathrm{~mol} \%$ ), and AgF (22.9 mg, $0.18 \mathrm{mmol})$. Then the reaction vial was repeatedly purged with argon, and added $1.0 \mathrm{~mL}$ of cyclohexane and $\mathbf{2 c}(69.6 \mathrm{mg}, 0.3 \mathrm{mmol})$ with syringe. The mixture was stirred in an oil bath at $80^{\circ} \mathrm{C}$ for $12 \mathrm{~h}$. After the reaction mixture had cooled down to room temperature, filtered through a short silica bed eluting with EtOAc. The solvent was evaporated under reduced pressure to leave a crude product, which was purified by 
flash column chromatography on silica gel eluting with PE/EtOAc (10:1) to afford the product 8.

\section{Characterization data for the products}

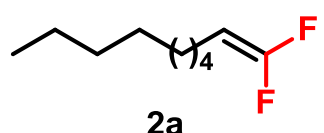

This compound was prepared according to the general procedure A. The crude mixture was purified by column chromatography with petroleum ether as the eluent to give the product $\mathbf{2 a}$ as a colorless liquid (34.8 mg, 79\% yield). ${ }^{1} \mathbf{H}$ NMR $\left(600 \mathrm{MHz}, \mathrm{CDCl}_{3}\right) \delta 4.12(\mathrm{dtd}, J=25.8,7.8,3.0 \mathrm{~Hz}, 1 \mathrm{H})$, 1.98-1.94 (m, 2H), 1.37-1.27 (m, 12H), $0.88(\mathrm{t}, J=6.6 \mathrm{~Hz}, 3 \mathrm{H}) .{ }^{13} \mathbf{C}$ NMR $\left(151 \mathrm{MHz}, \mathrm{CDCl}_{3}\right) \delta$ $156.3(\mathrm{dd}, J=285.4,283.9 \mathrm{~Hz}), 78.0(\mathrm{t}, J=21.1 \mathrm{~Hz}), 31.9,29.5(\mathrm{t}, J=6.0 \mathrm{~Hz}), 29.31,29.45,28.9$, 22.7, $22.1(\mathrm{~d}, J=3.0 \mathrm{~Hz}), 14.1 .{ }^{19} \mathbf{F}$ NMR $\left(470 \mathrm{MHz}, \mathrm{CDCl}_{3}\right) \delta-89.90(\mathrm{~d}, J=49.8 \mathrm{~Hz}),-92.29(\mathrm{dd}$, $J=49.8,25.8 \mathrm{~Hz}) . \mathbf{I R}\left(\right.$ film): 2921, 2851, 1748, 1457, 1225, 803, 720, $669 \mathrm{~cm}^{-1} . \mathbf{M S}(\mathrm{EI}, \mathrm{m} / \mathrm{z}): 176$ $\left(\mathrm{M}^{+}, 100\right)$.

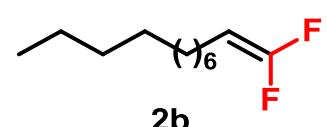

This compound was prepared according to the general procedure A. The crude mixture was purified by column chromatography with petroleum ether as the eluent to give the product $\mathbf{2 b}$ as a colorless liquid (41.8 mg, 82\% yield). ${ }^{1} \mathbf{H}$ NMR $\left(600 \mathrm{MHz}, \mathrm{CDCl}_{3}\right) \delta 4.12(\mathrm{dtd}, J=25.8,8.4,3.0 \mathrm{~Hz}, 1 \mathrm{H})$, 1.98-1.94 (m, 2H), 1.37-1.26 (m, 16H), $0.88(\mathrm{t}, J=7.2 \mathrm{~Hz}, 3 \mathrm{H}) .{ }^{13} \mathbf{C}$ NMR $\left(151 \mathrm{MHz}, \mathrm{CDCl}_{3}\right) \delta$ $156.3(\mathrm{dd}, J=285.4,283.9 \mathrm{~Hz}), 78.1(\mathrm{t}, J=21.1 \mathrm{~Hz}), 31.9,29.6,29.4(\mathrm{t}, J=1.5 \mathrm{~Hz}), 29.34,29.33$, 28.9, 22.7, $22.1(\mathrm{~d}, J=4.2 \mathrm{~Hz}), 14.1 .{ }^{19} \mathbf{F}$ NMR $\left(470 \mathrm{MHz}, \mathrm{CDCl}_{3}\right) \delta-89.88(\mathrm{~d}, J=49.8 \mathrm{~Hz}),-92.26$ (dd, $J=49.8,25.8 \mathrm{~Hz}$ ). IR (film): 2922, 2852, 1747, 1458, 1261, 1018, 750, $669 \mathrm{~cm}^{-1}$. MS (EI, $\mathrm{m} / \mathrm{z}): 204\left(\mathrm{M}^{+}, 100\right)$.

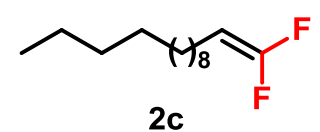

This compound was prepared according to the general procedure A. The crude mixture was purified by column chromatography with petroleum ether as the eluent to give the product $\mathbf{2 c}$ as a colorless liquid (49.3 mg, 85\% yield). ${ }^{1} \mathbf{H}$ NMR $\left(600 \mathrm{MHz}, \mathrm{CDCl}_{3}\right) \delta 4.11(\mathrm{dtd}, J=25.8,8.4,3.0 \mathrm{~Hz}, 1 \mathrm{H})$, $1.98-1.94(\mathrm{~m}, 2 \mathrm{H}), 1.37-1.26(\mathrm{~m}, 20 \mathrm{H}), 0.88(\mathrm{t}, J=6.6 \mathrm{~Hz}, 3 \mathrm{H}) .{ }^{13} \mathbf{C} \mathbf{~ N M R}\left(151 \mathrm{MHz}, \mathrm{CDCl}_{3}\right) \delta$ $156.3(\mathrm{dd}, J=286.9,283.9 \mathrm{~Hz}), 78.0(\mathrm{t}, J=21.1 \mathrm{~Hz}), 31.9,29.72,29.68,29.65,29.65,29.60,29.5$ 
$(\mathrm{t}, J=1.5 \mathrm{~Hz}), 29.4(\mathrm{~d}, J=3.0 \mathrm{~Hz}), 28.9,22.7,22.1(\mathrm{~d}, J=4.5 \mathrm{~Hz}), 14.1 .{ }^{19} \mathbf{F}$ NMR $(470 \mathrm{MHz}$, $\left.\mathrm{CDCl}_{3}\right) \delta-89.89(\mathrm{~d}, J=49.4 \mathrm{~Hz}$ ), -92.27 (dd, $J=49.4,25.8 \mathrm{~Hz}$ ). IR (film): 2926, 2855, 1747, 1457, 1224, 802, 720, $669 \mathrm{~cm}^{-1}$. MS (EI, m/z): $232\left(\mathrm{M}^{+}, 100\right)$.

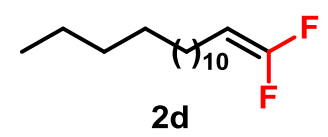

This compound was prepared according to the general procedure A. The crude mixture was purified by column chromatography with petroleum ether as the eluent to give the product $\mathbf{2 d}$ as a colorless liquid (58.5 mg, 90\% yield). ${ }^{1} \mathbf{H}$ NMR $\left(600 \mathrm{MHz}, \mathrm{CDCl}_{3}\right) \delta 4.11$ (dtd, $\left.J=25.8,8.4,2.4 \mathrm{~Hz}, 1 \mathrm{H}\right)$, $1.98-1.93(\mathrm{~m}, 2 \mathrm{H}), 1.37-1.26(\mathrm{~m}, 24 \mathrm{H}), 0.88(\mathrm{t}, J=7.2 \mathrm{~Hz}, 3 \mathrm{H}) .{ }^{13} \mathbf{C} \mathbf{N M R}\left(151 \mathrm{MHz}, \mathrm{CDCl}_{3}\right) \delta$ $156.3(\mathrm{dd}, J=286.4,284.5 \mathrm{~Hz}), 78.0(\mathrm{t}, J=21.1 \mathrm{~Hz}), 32.0,29.76,29.75,29.73,29.71,29.68,29.63$, $29.5(\mathrm{t}, J=1.5 \mathrm{~Hz}), 29.41,29.38,29.0,22.7,22.2(\mathrm{~d}, J=4.1 \mathrm{~Hz}), 14.1 .{ }^{19} \mathbf{F} \mathbf{N M R}\left(470 \mathrm{MHz}, \mathrm{CDCl}_{3}\right)$ $\delta$-91.83 (d, $J=49.4 \mathrm{~Hz}$ ), -94.22 (dd, $J=49.4,25.8 \mathrm{~Hz}$ ). IR (film): 2925, 2854, 1746, 1457, 1307, 1217, 801, 720, $669 \mathrm{~cm}^{-1}$. MS (EI, m/z): $226.34\left(\mathrm{M}^{+}, 100.00\right)$.

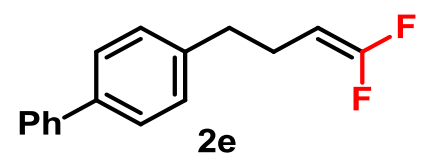

This compound was prepared according to the general procedure A. The crude mixture was purified by column chromatography with petroleum ether as the eluent to give the product $\mathbf{2 e}$ as a colorless liquid (54.9 mg, 90\% yield). ${ }^{1} \mathbf{H}$ NMR $\left(600 \mathrm{MHz}, \mathrm{CDCl}_{3}\right) \delta 7.58(\mathrm{~d}, J=7.2 \mathrm{~Hz}, 2 \mathrm{H}), 7.52(\mathrm{~d}, J=$ $7.8 \mathrm{~Hz}, 2 \mathrm{H}), 7.42(\mathrm{t}, J=7.8 \mathrm{~Hz}, 2 \mathrm{H}), 7.32(\mathrm{t}, J=7.8 \mathrm{~Hz}, 1 \mathrm{H}), 7.24(\mathrm{~d}, J=7.8 \mathrm{~Hz}, 2 \mathrm{H}), 4.18(\mathrm{dtd}, J$ $=25.2,7.8,1.8 \mathrm{~Hz}, 1 \mathrm{H}), 2.72(\mathrm{t}, J=7.8 \mathrm{~Hz}, 2 \mathrm{H}), 2.33(\mathrm{q}, J=7.8 \mathrm{~Hz}, 2 \mathrm{H}) .{ }^{13} \mathbf{C} \mathbf{N M R}(151 \mathrm{MHz}$, $\left.\mathrm{CDCl}_{3}\right) \delta 156.3(\mathrm{dd}, J=287.0,285.4 \mathrm{~Hz}), 141.0,140.0,139.1,128.8,128.7,127.1,127.1,127.0$, $77.3(\mathrm{dd}, J=21.9,22.7 \mathrm{~Hz}), 35.3(\mathrm{t}, J=2.2 \mathrm{~Hz}), 24.0(\mathrm{~d}, J=4.5 \mathrm{~Hz}) .{ }^{19} \mathbf{F} \mathbf{N M R}\left(565 \mathrm{MHz}, \mathrm{CDCl}_{3}\right)$ $\delta-88.77(\mathrm{~d}, J=47.5 \mathrm{~Hz}),-90.79(\mathrm{dd}, J=47.5,25.4 \mathrm{~Hz})$. The spectral data is consistent with the reported literature. ${ }^{3}$

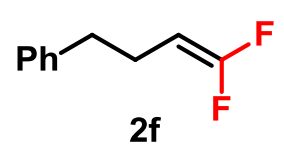

This compound was prepared according to the general procedure A. The crude mixture was purified by column chromatography with petroleum ether as the eluent to give the product $\mathbf{2} \mathbf{f}$ as a colorless liquid (39.5 mg, 94\% yield). ${ }^{1} \mathbf{H}$ NMR $\left(600 \mathrm{MHz}, \mathrm{CDCl}_{3}\right) \delta 7.27(\mathrm{t}, J=7.8 \mathrm{~Hz}, 2 \mathrm{H}), 7.18(\mathrm{t}, J=7.2$ 
$\mathrm{Hz}, 1 \mathrm{H}), 7.15(\mathrm{~d}, J=7.2 \mathrm{~Hz}, 2 \mathrm{H}), 4.12(\mathrm{dtd}, J=25.2,7.8,2.4 \mathrm{~Hz}, 1 \mathrm{H}), 2.65(\mathrm{t}, J=7.2 \mathrm{~Hz}, 2 \mathrm{H})$, 2.30-2.25 (m, 2H). ${ }^{13}$ C NMR (151 MHz, $\left.\mathrm{CDCl}_{3}\right) \delta 156.4(\mathrm{dd}, J=288.4,285.4 \mathrm{~Hz}), 140.9,128.41$, 128.38, 126.1, $77.3(\mathrm{dd}, J=22.7,21.4 \mathrm{~Hz}), 35.7(\mathrm{t}, J=1.5 \mathrm{~Hz}), 24.0(\mathrm{~d}, J=4.5 \mathrm{~Hz}) .{ }^{19} \mathbf{F}$ NMR $(470$ $\left.\mathrm{MHz}, \mathrm{CDCl}_{3}\right) \delta-88.99(\mathrm{~d}, J=47.5 \mathrm{~Hz}),-91.06(\mathrm{dd}, J=47.5,25.2 \mathrm{~Hz})$. The spectral data is consistent with the reported literature. ${ }^{4}$

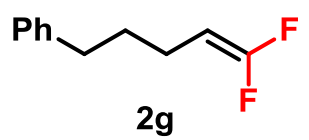

This compound was prepared according to the general procedure A. The crude mixture was purified by column chromatography with petroleum ether as the eluent to give the product $\mathbf{2 g}$ as a colorless liquid (41.8 mg, 92\% yield). ${ }^{1} \mathbf{H}$ NMR $\left(600 \mathrm{MHz}, \mathrm{CDCl}_{3}\right) \delta 7.28(\mathrm{t}, J=7.8 \mathrm{~Hz}, 2 \mathrm{H}), 7.21-7.14(\mathrm{~m}$, 3H), $4.15(\mathrm{dtd}, J=25.2,7.8,2.4 \mathrm{~Hz}, 1 \mathrm{H}), 2.62(\mathrm{t}, J=7.8 \mathrm{~Hz}, 2 \mathrm{H}), 2.01(\mathrm{q}, J=7.2 \mathrm{~Hz}, 2 \mathrm{H}), 1.72-$ $1.67(\mathrm{~m}, 2 \mathrm{H}) .{ }^{13} \mathrm{C} \mathrm{NMR}\left(151 \mathrm{MHz}, \mathrm{CDCl}_{3}\right) \delta 156.4(\mathrm{dd}, J=286.9,284.8 \mathrm{~Hz}), 141.9,128.41,128.37$, 125.9, $77.7(\mathrm{t}, J=21.1 \mathrm{~Hz}), 35.2,31.1(\mathrm{t}, J=3.0 \mathrm{~Hz}), 21.8(\mathrm{~d}, J=4.5 \mathrm{~Hz}) .{ }^{19} \mathbf{F} \mathbf{N M R}(470 \mathrm{MHz}$, $\left.\mathrm{CDCl}_{3}\right) \delta-89.19(\mathrm{~d}, J=48.0 \mathrm{~Hz}),-91.50(\mathrm{dd}, J=48.0,25.4 \mathrm{~Hz})$. The spectral data is consistent with the reported literature. ${ }^{5}$

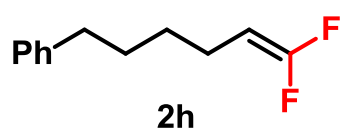

This compound was prepared according to the general procedure A. The crude mixture was purified by column chromatography with petroleum ether as the eluent to give the product $\mathbf{2 h}$ as a colorless liquid (44.1 mg, 90\% yield). ${ }^{1} \mathbf{H}$ NMR (600 MHz, $\left.\mathrm{CDCl}_{3}\right) \delta 7.28(\mathrm{t}, J=7.2 \mathrm{~Hz}, 2 \mathrm{H}), 7.20-7.16(\mathrm{~m}$, 3H), $4.11(\mathrm{dtd}, J=25.8,7.8,3.0 \mathrm{~Hz}, 1 \mathrm{H}), 2.61(\mathrm{t}, J=7.8 \mathrm{~Hz}, 2 \mathrm{H}), 2.02-1.96(\mathrm{~m}, 2 \mathrm{H}), 1.66-1.60(\mathrm{~m}$, 2H), 1.44-1.38 (m, 2H). ${ }^{13} \mathbf{C}$ NMR (151 MHz, $\left.\mathrm{CDCl}_{3}\right) \delta 156.3$ (dd, $\left.J=286.7,285.0 \mathrm{~Hz}\right), 142.4$, 128.4, 128.3, 125.8, $77.9(\mathrm{t}, J=21.2 \mathrm{~Hz}), 35.6,30.7,29.0(\mathrm{t}, J=2.4 \mathrm{~Hz}), 22.0(\mathrm{~d}, J=4.1 \mathrm{~Hz}) .{ }^{19} \mathbf{F}$ NMR $\left(470 \mathrm{MHz}, \mathrm{CDCl}_{3}\right) \delta-89.52(\mathrm{~d}, J=48.8 \mathrm{~Hz}),-91.93(\mathrm{dd}, J=48.8,25.8 \mathrm{~Hz})$. The spectral data is consistent with the reported literature. ${ }^{5}$<smiles>CC(CC=C(F)F)c1ccccc1</smiles>

This compound was prepared according to the general procedure A. The crude mixture was purified by column chromatography with petroleum ether as the eluent to give the product $\mathbf{2 i}$ as a colorless 
liquid (37.3 mg, 82\% yield). ${ }^{1} \mathbf{H}$ NMR $\left(600 \mathrm{MHz}, \mathrm{CDCl}_{3}\right) \delta 7.30(\mathrm{t}, J=7.2 \mathrm{~Hz}, 2 \mathrm{H}), 7.22-7.16(\mathrm{~m}$, $3 \mathrm{H}), 4.04(\mathrm{dtd}, J=25.8,7.8,3.0 \mathrm{~Hz}, 1 \mathrm{H}), 2.78-2.73(\mathrm{~m}, 1 \mathrm{H}), 2.27-2.21(\mathrm{~m}, 2 \mathrm{H}), 1.27(\mathrm{~d}, J=7.8 \mathrm{~Hz}$, 3H). ${ }^{13} \mathrm{C}$ NMR $\left(151 \mathrm{MHz}, \mathrm{CDCl}_{3}\right) \delta 156.5(\mathrm{dd}, J=286.9,285.4 \mathrm{~Hz}), 146.0,128.4,126.9,126.3$, $76.4(\mathrm{dd}, J=21.1,20.8 \mathrm{~Hz}), 39.8(\mathrm{t}, J=1.5 \mathrm{~Hz}), 30.9(\mathrm{~d}, J=4.5 \mathrm{~Hz}), 21.2 .{ }^{19} \mathbf{F}$ NMR $(470 \mathrm{MHz}$, $\left.\mathrm{CDCl}_{3}\right) \delta-88.68(\mathrm{~d}, J=47.0 \mathrm{~Hz}),-91.11(\mathrm{dd}, J=47.0,25.8 \mathrm{~Hz})$. The spectral data is consistent with the reported literature. ${ }^{6}$<smiles>FC(F)=CCC(c1ccccc1)c1ccccc1</smiles>

This compound was prepared according to the general procedure A. The crude mixture was purified by column chromatography with petroleum ether as the eluent to give the product $\mathbf{2} \mathbf{j}$ as a colorless liquid (39.7 mg, 65\% yield). ${ }^{1} \mathrm{H}$ NMR $\left(600 \mathrm{MHz}, \mathrm{CDCl}_{3}\right) \delta 7.28(\mathrm{t}, J=7.8 \mathrm{~Hz}, 4 \mathrm{H}), 7.24-7.17(\mathrm{~m}, 6 \mathrm{H})$, $4.07(\mathrm{dtd}, J=25.4,7.7,2.4 \mathrm{~Hz}, 1 \mathrm{H}), 3.95(\mathrm{t}, J=8.4 \mathrm{~Hz}, 1 \mathrm{H}), 2.72(\mathrm{t}, J=7.8 \mathrm{~Hz}, 2 \mathrm{H}) .{ }^{13} \mathbf{C} \mathbf{N M R}(150$ $\left.\mathrm{MHz}, \mathrm{CDCl}_{3}\right) \delta 156.3(\mathrm{dd}, J=286.5,285.0 \mathrm{~Hz}), 143.7,128.5,127.8,126.5,76.6(\mathrm{dd}, J=19.6,22.7 \mathrm{~Hz})$, 51.1, $28.5(\mathrm{~d}, J=4.5 \mathrm{~Hz}) .{ }^{19} \mathbf{F}$ NMR $\left(470 \mathrm{MHz}, \mathrm{CDCl}_{3}\right) \delta-88.21(\mathrm{~d}, J=45.6 \mathrm{~Hz}),-90.13(\mathrm{dd}, J=45.6$, $25.4 \mathrm{~Hz})$. The spectral data is consistent with the reported literature. ${ }^{4}$

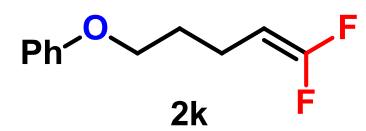

This compound was prepared according to the general procedure A. The crude mixture was purified by column chromatography with petroleum ether as the eluent to give the product $\mathbf{2 k}$ as a colorless liquid (41.3 mg, 85\% yield). ${ }^{1} \mathrm{H}$ NMR (600 MHz, $\left.\mathrm{CDCl}_{3}\right) \delta 7.28(\mathrm{t}, J=7.2 \mathrm{~Hz}, 2 \mathrm{H}), 6.94(\mathrm{t}, J=7.8 \mathrm{~Hz}$, 1H), $6.89(\mathrm{~d}, J=7.8 \mathrm{~Hz}, 2 \mathrm{H}), 4.19(\mathrm{dtd}, J=25.2,7.8,2.4 \mathrm{~Hz}, 1 \mathrm{H}), 3.96(\mathrm{t}, J=6.0 \mathrm{~Hz}, 2 \mathrm{H}), 2.21-2.15$ (m, 2H), 1.89-1.83 (m, 2H). ${ }^{13} \mathbf{C}$ NMR $\left(151 \mathrm{MHz}, \mathrm{CDCl}_{3}\right) \delta 158.9,156.4(\mathrm{dd}, J=288.4,285.4 \mathrm{~Hz})$, 129.4, 120.7, 114.5, $77.3(\mathrm{t}, J=21.1 \mathrm{~Hz}), 66.7,29.0(\mathrm{t}, J=2.4 \mathrm{~Hz}), 18.9$ (d, $J=4.5 \mathrm{~Hz}) .{ }^{19} \mathbf{F}$ NMR $(470$ $\left.\mathrm{MHz}, \mathrm{CDCl}_{3}\right) \delta-88.73(\mathrm{~d}, J=47.5 \mathrm{~Hz}),-91.16(\mathrm{dd}, J=47.5,25.2 \mathrm{~Hz})$. The spectral data is consistent with the reported literature. ${ }^{5}$<smiles>FC(F)=CCCCSc1ccccc1</smiles>

This compound was prepared according to the general procedure A. The crude mixture was purified by column chromatography with petroleum ether as the eluent to give the product $\mathbf{2 l}$ as a colorless 
liquid (38.5 mg, $72 \%$ yield). ${ }^{1} \mathbf{H} \mathbf{~ N M R}\left(600 \mathrm{MHz}, \mathrm{CDCl}_{3}\right) \delta 7.33(\mathrm{~d}, J=7.2 \mathrm{~Hz}, 2 \mathrm{H}), 7.28(\mathrm{t}, J=7.8$ $\mathrm{Hz}, 2 \mathrm{H}), 7.18(\mathrm{t}, J=7.8 \mathrm{~Hz}, 1 \mathrm{H}), 4.12(\mathrm{dtd}, J=25.2,7.8,2.4 \mathrm{~Hz}, 1 \mathrm{H}), 2.91(\mathrm{t}, J=7.2 \mathrm{~Hz}, 2 \mathrm{H}), 2.14-2.09$ (m, 2H), 1.73-1.68 (m, 2H). ${ }^{13} \mathbf{C}$ NMR $\left(151 \mathrm{MHz}, \mathrm{CDCl}_{3}\right) \delta 156.5(\mathrm{dd}, J=287.0,285.4 \mathrm{~Hz}), 136.3$, 129.3, 128.9, 126.0, $77.0(\mathrm{t}, J=22.7 \mathrm{~Hz}), 32.9,28.8(\mathrm{t}, J=3.0 \mathrm{~Hz}), 21.26(\mathrm{~d}, J=4.5 \mathrm{~Hz}) .{ }^{19} \mathbf{F}$ NMR $(470$ MHz, $\left.\mathrm{CDCl}_{3}\right) \delta-88.49$ (d, $\left.J=47.0 \mathrm{~Hz}\right),-90.89$ (dd, $J=47.0,25.2 \mathrm{~Hz}$ ). IR (film): 3075, 2930, 2862, 1747, 1541, 1306, 1149, 1086, $689 \mathrm{~cm}^{-1} . \mathbf{M S}(\mathrm{EI}, \mathrm{m} / \mathrm{z}): 214.17\left(\mathrm{M}^{+}, 55.11\right), 123.11$ (73.05), 110.13 (100.00), 77.07 (30.87).

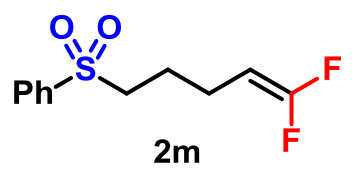

This compound was prepared according to the general procedure A. The crude mixture was purified by column chromatography with ethyl acetate/petroleum ether $=1: 10$ as the eluent to give the product $\mathbf{2 m}$ as a yellow liquid (38.1 $\mathrm{mg}, 62 \%$ yield). ${ }^{1} \mathbf{H} \mathbf{~ N M R ~}\left(600 \mathrm{MHz}, \mathrm{CDCl}_{3}\right) \delta 7.91$ (d, $J=7.8$ $\mathrm{Hz}, 2 \mathrm{H}), 7.68(\mathrm{t}, J=7.2 \mathrm{~Hz}, 1 \mathrm{H}), 7.59(\mathrm{t}, J=7.8 \mathrm{~Hz}, 2 \mathrm{H}), 4.08(\mathrm{dtd}, J=25.2,7.8,1.8 \mathrm{~Hz}, 1 \mathrm{H}), 3.11-$ $3.06(\mathrm{~m}, 2 \mathrm{H}), 2.09(\mathrm{q}, J=7.2 \mathrm{~Hz}, 2 \mathrm{H}), 1.85-1.78(\mathrm{~m}, 2 \mathrm{H}) .{ }^{13} \mathbf{C} \mathbf{N M R}\left(151 \mathrm{MHz}, \mathrm{CDCl}_{3}\right) \delta 156.6$ (dd, $J=288.4,286.9 \mathrm{~Hz}), 139.0,133.8,129.3,128.0,76.3(\mathrm{dd}, J=22.7,21.1 \mathrm{~Hz}), 55.3,22.5(\mathrm{t}, J=$ $1.5 \mathrm{~Hz}), 20.9(\mathrm{~d}, J=4.5 \mathrm{~Hz}) .{ }^{19} \mathbf{F} \mathbf{N M R}\left(470 \mathrm{MHz}, \mathrm{CDCl}_{3}\right) \delta-87.31(\mathrm{~d}, J=44.2 \mathrm{~Hz}),-89.89(\mathrm{dd}, J$ $=44.2,25.2 \mathrm{~Hz}) . \mathbf{I R}$ (film): 3065, 2927, 1747, 1457, 1224, 802, 720, $669 \mathrm{~cm}^{-1} . \mathbf{M S}(\mathrm{EI}, \mathrm{m} / \mathrm{z}): 246.17$ $\left(\mathrm{M}^{+}, 17.54\right), 181.20(100.00)$.

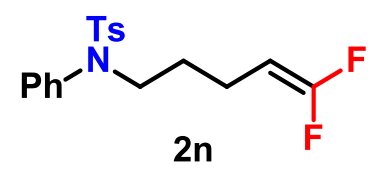

This compound was prepared according to the general procedure A. The crude mixture was purified by column chromatography with ethyl acetate/petroleum ether $=6: 1$ as the eluent to give the product 2n as a yellow liquid $\left(59.7 \mathrm{mg}, 68 \%\right.$ yield). ${ }^{1} \mathbf{H}$ NMR $\left(600 \mathrm{MHz}, \mathrm{CDCl}_{3}\right) \delta 7.45(\mathrm{~d}, J=8.4 \mathrm{~Hz}, 2 \mathrm{H})$, 7.33-7.29 (m, 3H), 7.24 (d, $J=8.4 \mathrm{~Hz}, 2 \mathrm{H}), 7.06-7.00(\mathrm{~m}, 2 \mathrm{H}), 4.11(\mathrm{dtd}, J=25.2,7.8,2.4 \mathrm{~Hz}, 1 \mathrm{H})$, $3.54(\mathrm{t}, J=7.2 \mathrm{~Hz}, 2 \mathrm{H}), 2.42(\mathrm{~s}, 3 \mathrm{H}), 2.06-2.00(\mathrm{~m}, 2 \mathrm{H}), 1.51-1.46(\mathrm{~m}, 2 \mathrm{H}) .{ }^{13} \mathbf{C}$ NMR $(151 \mathrm{MHz}$, $\left.\mathrm{CDCl}_{3}\right) \delta 156.3(\mathrm{dd}, J=287.0,285.4 \mathrm{~Hz}), 143.4,138.9,135.0,129.3,129.0,128.7,127.9,127.6$, $76.9(\mathrm{dd}, J=22.4,20.7 \mathrm{~Hz}), 49.5,27.9(\mathrm{t}, J=2.0 \mathrm{~Hz}), 21.5,19.1(\mathrm{~d}, J=4.5 \mathrm{~Hz}) .{ }^{19} \mathbf{F} \mathbf{N M R}(470$ $\left.\mathrm{MHz}, \mathrm{CDCl}_{3}\right) \delta-88.55$ (d, $J=46.5 \mathrm{~Hz}$ ), -90.87 (dd, $\left.J=46.5,25.2 \mathrm{~Hz}\right)$. IR (film): 3065, 2927, 2868, 
1747, 1490, 1349, 1162, $573 \mathrm{~cm}^{-1}$. MS (EI, m/z): $351.25\left(\mathrm{M}^{+}, 30.75\right), 260.18(31.97), 196.17$ (44.38), $155.09(100.00)$.

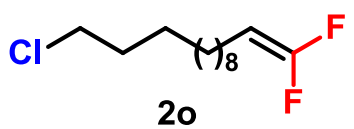

This compound was prepared according to the general procedure A. The crude mixture was purified by column chromatography with petroleum ether as the eluent to give the product $\mathbf{2 0}$ as a colorless liquid (44.7 mg, 71\% yield).; ${ }^{1} \mathbf{H}$ NMR (600 MHz, $\left.\mathrm{CDCl}_{3}\right) \delta 4.11(\mathrm{dtd}, J=25.8,7.8,2.4 \mathrm{~Hz}, 1 \mathrm{H})$, $3.53(\mathrm{t}, J=6.8 \mathrm{~Hz}, 2 \mathrm{H}), 1.97(\mathrm{q}, J=6.6 \mathrm{~Hz}, 2 \mathrm{H}), 1.79-1.74(\mathrm{~m}, 2 \mathrm{H}), 1.45-1.26(\mathrm{~m}, 16 \mathrm{H}) .{ }^{13} \mathbf{C}$ NMR $\left(151 \mathrm{MHz}, \mathrm{CDCl}_{3}\right) \delta 156.26(\mathrm{dd}, J=286.9,285.4 \mathrm{~Hz}), 78.00(\mathrm{t}, J=21.1 \mathrm{~Hz}), 45.1,32.7,29.51$, 29.49, 29.44, 29.42, 29.41, 29.30, $28.9(\mathrm{~d}, J=1.1 \mathrm{~Hz}), 26.9,22.1(\mathrm{~d}, J=3.0 \mathrm{~Hz}) .{ }^{19} \mathbf{F}$ NMR $(470$ MHz, $\mathrm{CDCl}_{3}$ ) $\delta$-90.04 (d, $J=49.8 \mathrm{~Hz}$ ), -92.44 (dd, $J=49.8,25.8 \mathrm{~Hz}$ ). IR (film): 2928, 2856, 1748, 1747, 1308, 1214, $742 \mathrm{~cm}^{-1}$. MS (EI, m/z): $252.22\left(\mathrm{M}^{+}, 6.92\right), 232.22$ (14.26), 224.20 (23.33), 188.22 (32.74), $175.22(100.00)$.

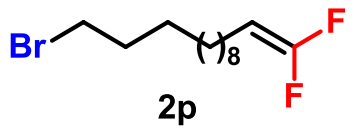

This compound was prepared according to the general procedure A. The crude mixture was purified by column chromatography with petroleum ether as the eluent to give the product $\mathbf{2 p}$ as a colorless liquid (61.4 mg, 83\% yield). ${ }^{1} \mathbf{H}$ NMR $\left(600 \mathrm{MHz}, \mathrm{CDCl}_{3}\right) \delta 4.12(\mathrm{dtd}, J=25.8,7.8,3.0 \mathrm{~Hz}, 1 \mathrm{H})$, $3.40(\mathrm{t}, J=7.2 \mathrm{~Hz}, 2 \mathrm{H}), 1.98-1.94(\mathrm{~m}, 2 \mathrm{H}), 1.88-1.83(\mathrm{~m}, 2 \mathrm{H}), 1.43-1.27(\mathrm{~m}, 16 \mathrm{H}) .{ }^{13} \mathrm{C}$ NMR $(151$ $\left.\mathrm{MHz}, \mathrm{CDCl}_{3}\right) \delta 156.25(\mathrm{dd}, J=285.4,284.9 \mathrm{~Hz}), 78.0(\mathrm{t}, J=21.1 \mathrm{~Hz}), 34.0,32.9,29.52,29.50$, 29.45, 29.43, 29.3, 28.9, 28.8, 28.2, $22.1(\mathrm{~d}, J=4.5 \mathrm{~Hz}) .{ }^{19} \mathbf{F}$ NMR $\left(470 \mathrm{MHz}, \mathrm{CDCl}_{3}\right) \delta-89.83(\mathrm{~d}$, $J=49.8 \mathrm{~Hz}$ ), -92.24 (dd, $J=49.8,25.8 \mathrm{~Hz}$ ). IR (film): 2928, 2856, 1746, 1464, 1304, 1212, 913 , 803, 722, $647 \mathrm{~cm}^{-1}$. MS (EI, m/z): $296.19\left(\mathrm{M}^{+}, 23.20\right), 276.18$ (100.00).

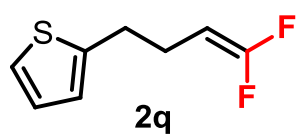

This compound was prepared according to the general procedure A. The crude mixture was purified by column chromatography with petroleum ether as the eluent to give the product $\mathbf{2 q}$ as a colorless liquid (35.2 mg, 81\% yield). ${ }^{1} \mathbf{H}$ NMR $\left(600 \mathrm{MHz}, \mathrm{CDCl}_{3}\right) \delta 7.13(\mathrm{dd}, J=5.1,1.2 \mathrm{~Hz}, 1 \mathrm{H}), 6.92(\mathrm{dd}$, $J=5.1,4.8 \mathrm{~Hz}, 1 \mathrm{H}), 6.80-6.79(\mathrm{~m}, 1 \mathrm{H}), 4.19(\mathrm{dtd}, J=25.2,7.8,2.4 \mathrm{~Hz}, 1 \mathrm{H}), 2.90(\mathrm{t}, J=7.8 \mathrm{~Hz}$, 2H), 2.38-2.32 (m, 2H). ${ }^{13} \mathbf{C}$ NMR $\left(151 \mathrm{MHz}, \mathrm{CDCl}_{3}\right) \delta 156.5(\mathrm{dd}, J=288.1,285.6 \mathrm{~Hz}), 143.5$, 
126.8, 124.5, 123.4, $77.0(\mathrm{t}, J=21.1 \mathrm{~Hz}), 29.7(\mathrm{t}, J=3.0 \mathrm{~Hz}), 24.4(\mathrm{~d}, J=4.5 \mathrm{~Hz}) .{ }^{19} \mathbf{F} \mathbf{N M R}(470$ $\left.\mathrm{MHz}, \mathrm{CDCl}_{3}\right) \delta-88.44(\mathrm{~d}, J=46.1 \mathrm{~Hz}),-90.39$ (dd, $\left.J=46.1,25.2 \mathrm{~Hz}\right)$. IR (film): 2921, 28501541 , $1151,669 \mathrm{~cm}^{-1} . \mathbf{M S}(\mathrm{EI}, \mathrm{m} / \mathrm{z}): 174.14\left(\mathrm{M}^{+}, 13.52\right), 97.10$ (100.00).

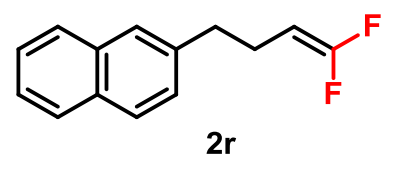

This compound was prepared according to the general procedure A. The crude mixture was purified by column chromatography with petroleum ether as the eluent to give the product $\mathbf{2 r}$ as a colorless liquid (45.8 mg, 84\% yield). ${ }^{1} \mathbf{H}$ NMR (600 MHz, $\left.\mathrm{CDCl}_{3}\right) \delta 7.82-7.75(\mathrm{~m}, 3 \mathrm{H}), 7.60$ (s, 1H), 7.46$7.41(\mathrm{~m}, 2 \mathrm{H}), 7.31(\mathrm{~d}, J=8.4 \mathrm{~Hz}, 1 \mathrm{H}), 4.17(\mathrm{dtd}, J=25.8,7.8,2.4 \mathrm{~Hz}, 1 \mathrm{H}), 2.84(\mathrm{t}, J=7.2 \mathrm{~Hz}, 2 \mathrm{H})$, 2.41-2.35 (m, 2H). ${ }^{13} \mathrm{C}$ NMR (151 MHz, $\left.\mathrm{CDCl}_{3}\right) \delta 156.3(\mathrm{dd}, J=288.4,285.4 \mathrm{~Hz}), 138.4,133.6$, $132.1,128.0,127.6,127.5,127.1,126.6,126.0,125.3,77.3(\mathrm{dd}, \mathrm{J}=22.1,20.9 \mathrm{~Hz}), 35.8(\mathrm{t}, J=3.0$ $\mathrm{Hz}), 23.9(\mathrm{~d}, J=4.5 \mathrm{~Hz}) .{ }^{19} \mathbf{F}$ NMR $\left(470 \mathrm{MHz}, \mathrm{CDCl}_{3}\right) \delta-88.80(\mathrm{~d}, J=47.0 \mathrm{~Hz}),-90.84(\mathrm{dd}, J=$ 47.0, $25.8 \mathrm{~Hz}$ ). The spectral data is consistent with the reported literature. ${ }^{6}$

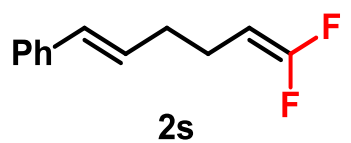

This compound was prepared according to the general procedure A. The crude mixture was purified by column chromatography with petroleum ether as the eluent to give the product $2 \mathbf{s}$ as a colorless liquid (33.9 mg, 70\% yield). ${ }^{\mathbf{1}} \mathbf{H}$ NMR $\left(600 \mathrm{MHz}, \mathrm{CDCl}_{3}\right) \delta 7.33(\mathrm{~d}, J=7.2 \mathrm{~Hz}, 2 \mathrm{H}), 7.29(\mathrm{t}, J=$ $7.2 \mathrm{~Hz}, 2 \mathrm{H}), 7.20(\mathrm{t}, J=7.2 \mathrm{~Hz}, 1 \mathrm{H}), 6.40(\mathrm{~d}, J=16.2 \mathrm{~Hz}, 1 \mathrm{H}), 6.19-6.14(\mathrm{~m}, 1 \mathrm{H}), 4.18(\mathrm{dtd}, J=$ 25.8, 7.8, 2.4 Hz, 1H), $2.27(\mathrm{q}, J=7.2 \mathrm{~Hz}, 2 \mathrm{H}), 2.18-2.12(\mathrm{~m}, 2 \mathrm{H}) .{ }^{13} \mathbf{C} \mathbf{N M R}\left(151 \mathrm{MHz}, \mathrm{CDCl}_{3}\right) \delta$ $156.3(\mathrm{dd}, J=287.0,285.4 \mathrm{~Hz}), 137.5,131.0,129.0,128.5,127.1,126.0,77.3(\mathrm{t}, J=21.1 \mathrm{~Hz}), 32.8$ $(\mathrm{t}, J=1.5 \mathrm{~Hz}), 22.1(\mathrm{~d}, J=4.5 \mathrm{~Hz}) .{ }^{19} \mathbf{F}$ NMR $\left(470 \mathrm{MHz}, \mathrm{CDCl}_{3}\right) \delta-88.97(\mathrm{~d}, J=47.5 \mathrm{~Hz}),-91.04$ (dd, $J=47.5,25.8 \mathrm{~Hz})$. IR (film): 3027, 2928, 1747, 1313, 1221, 1165, 964, 741, $692 \mathrm{~cm}^{-1}$. MS (EI, m/z): $194.17\left(\mathrm{M}^{+}, 13.13\right), 117.18$ (100.00), 115.13 (56.70), 91.10 (12.80).

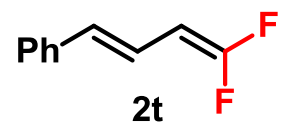

This compound was prepared according to the general procedure A. The crude mixture was purified by column chromatography with petroleum ether as the eluent to give the product $\mathbf{2 t}$ as a colorless liquid (59.7 mg, 60\% yield). ${ }^{\mathbf{1}} \mathbf{H}$ NMR $\left(600 \mathrm{MHz}, \mathrm{CDCl}_{3}\right) \delta 7.38(\mathrm{~d}, J=7.8 \mathrm{~Hz}, 2 \mathrm{H}), 7.31(\mathrm{t}, J=$ 
$7.8 \mathrm{~Hz}, 2 \mathrm{H}), 7.24(\mathrm{t}, J=7.2 \mathrm{~Hz}, 1 \mathrm{H}), 6.65(\mathrm{dd}, J=15.6,10.8 \mathrm{~Hz}, 1 \mathrm{H}), 6.48(\mathrm{~d}, J=15.6 \mathrm{~Hz}, 1 \mathrm{H})$, $5.13(\mathrm{dd}, J=24.0,10.8 \mathrm{~Hz}, 1 \mathrm{H}) .{ }^{13} \mathbf{C}$ NMR $\left(151 \mathrm{MHz}, \mathrm{CDCl}_{3}\right) \delta 156.9(\mathrm{dd}, J=297.5,291.4 \mathrm{~Hz})$, 136.9, $131.1(\mathrm{dd}, J=10.6,3.0 \mathrm{~Hz}), 128.7,127.7,126.2,117.9(\mathrm{dd}, J=4.5,3.0 \mathrm{~Hz}), 82.9(\mathrm{dd}, J=$ 27.2, 16.6 Hz). ${ }^{19} \mathbf{F}$ NMR $\left(470 \mathrm{MHz}, \mathrm{CDCl}_{3}\right) \delta-85.29(\mathrm{t}, J=25.9 \mathrm{~Hz}),-87.00(\mathrm{~d}, J=26.3 \mathrm{~Hz})$. The spectral data is consistent with the reported literature. ${ }^{4}$

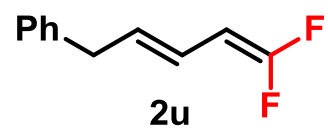

This compound was prepared according to the general procedure A. The crude mixture was purified by column chromatography with petroleum ether as the eluent to give the product $\mathbf{2 u}$ as a colorless liquid (66.9 mg, 61\% yield). ${ }^{1} \mathbf{H}$ NMR (600 MHz, $\left.\mathrm{CDCl}_{3}\right) \delta 7.29(\mathrm{t}, J=7.8 \mathrm{~Hz}, 2 \mathrm{H}), 7.21$ (t, $J=7.8$ $\mathrm{Hz}, 1 \mathrm{H}), 7.17(\mathrm{~d}, J=7.8 \mathrm{~Hz}, 2 \mathrm{H}), 6.01-5.97(\mathrm{~m}, 1 \mathrm{H}), 5.77-5.72(\mathrm{~m}, 1 \mathrm{H}), 4.93(\mathrm{dd}, J=24.6,10.8$ $\mathrm{Hz}, 1 \mathrm{H}), 3.41(\mathrm{~d}, J=7.2 \mathrm{~Hz}, 2 \mathrm{H}) .{ }^{13} \mathrm{C} \mathbf{N M R}\left(151 \mathrm{MHz}, \mathrm{CDCl}_{3}\right) \delta 156.1(\mathrm{dd}, J=296.0,289.9 \mathrm{~Hz})$, 139.8, $131.7(\mathrm{dd}, J=10.6,3.0 \mathrm{~Hz}), 128.5,128.3,126.2,119.9(\mathrm{dd}, J=4.5,1.5 \mathrm{~Hz}), 81.8(\mathrm{dd}, J=$ 27.2, $16.6 \mathrm{~Hz}), 39.2 .{ }^{19} \mathbf{F}$ NMR $\left(470 \mathrm{MHz}, \mathrm{CDCl}_{3}\right) \delta-86.99(\mathrm{dd}, J=32.4,24.4 \mathrm{~Hz}),-89.48(\mathrm{~d}, J=$ 32.4 Hz). IR (film): 2923, 2852, 1740, 1653, 1461, 1024 $\mathrm{cm}^{-1}$. MS (EI, m/z): 180 (M+, 79), 129 (100), 115(62).

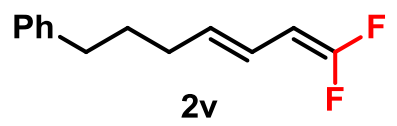

This compound was prepared according to the general procedure A. The crude mixture was purified by column chromatography with petroleum ether as the eluent to give the product $2 \mathbf{v}$ as a colorless liquid (93.3 mg, 67\% yield). ${ }^{1} \mathbf{H}$ NMR $\left(600 \mathrm{MHz}, \mathrm{CDCl}_{3}\right) \delta 7.27(\mathrm{t}, J=7.8 \mathrm{~Hz}, 2 \mathrm{H}), 7.20-7.15(\mathrm{~m}$, 3H), 5.94-5.90 (m, 1H), 5.63-5.58 (m, 1H), $4.90(\mathrm{dd}, J=24.6,10.8 \mathrm{~Hz}, 1 \mathrm{H}), 2.61(\mathrm{t}, J=7.2 \mathrm{~Hz}$, 2H), $2.11(\mathrm{q}, J=7.2 \mathrm{~Hz}, 2 \mathrm{H}), 1.74-1.69(\mathrm{~m}, 2 \mathrm{H}) .{ }^{13} \mathbf{C}$ NMR $\left(151 \mathrm{MHz}, \mathrm{CDCl}_{3}\right) \delta 155.9(\mathrm{dd}, J=$ 294.5, 288.4 Hz), 142.2, 133.0 (dd, $J=10.6,3.0 \mathrm{~Hz}), 128.4,128.3,125.8,119.0$ (dd, $J=3.0,1.5$ $\mathrm{Hz}), 82.0(\mathrm{dd}, J=27.2,16.6 \mathrm{~Hz}), 35.3,32.3,30.9 .{ }^{19} \mathbf{F} \mathbf{N M R}\left(470 \mathrm{MHz}, \mathrm{CDCl}_{3}\right) \delta-87.68(\mathrm{dd}, J=$ $34.8,24.6 \mathrm{~Hz}),-90.35(\mathrm{~d}, J=34.8 \mathrm{~Hz})$. The spectral data is consistent with the reported literature. ${ }^{7}$<smiles>FC(F)=Cc1ccc(-c2ccccc2)cc1</smiles> 
This compound was prepared according to the general procedure B. The crude mixture was purified by column chromatography with petroleum ether as the eluent to give the product $\mathbf{4 a}$ as a colorless liquid (56.4 mg, 87\% yield). ${ }^{1} \mathbf{H}$ NMR (600 MHz, $\left.\mathrm{CDCl}_{3}\right) \delta$ 7.59-7.55 (m, 4H), $7.43(\mathrm{t}, J=7.2 \mathrm{~Hz}$, 2H), $7.39(\mathrm{~d}, J=7.8 \mathrm{~Hz}, 2 \mathrm{H}), 7.34(\mathrm{t}, J=7.2 \mathrm{~Hz}, 1 \mathrm{H}), 5.30(\mathrm{dd}, J=26.6,3.0 \mathrm{~Hz}, 1 \mathrm{H}) .{ }^{13} \mathbf{C} \mathbf{N M R}$ $\left(151 \mathrm{MHz}, \mathrm{CDCl}_{3}\right) \delta 156.3(\mathrm{dd}, J=298.7,288.6 \mathrm{~Hz}), 140.5,139.8,129.4(\mathrm{t}, J=6.0 \mathrm{~Hz}), 128.8$, $128.0(\mathrm{dd}, J=6.0,3.0 \mathrm{~Hz}), 127.4,127.3,126.9,81.9(\mathrm{dd}, J=28.7,13.6 \mathrm{~Hz}) .{ }^{19} \mathbf{F}$ NMR $(565 \mathrm{MHz}$, $\left.\mathrm{CDCl}_{3}\right) \delta-81.90(\mathrm{dd}, J=30.5,26.6 \mathrm{~Hz}),-83.82(\mathrm{dd}, J=30.5,2.8 \mathrm{~Hz})$. The spectral data is consistent with the reported literature. ${ }^{8}$

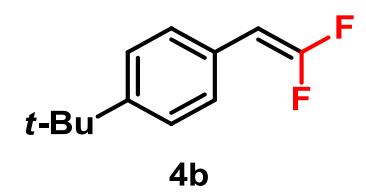

This compound was prepared according to the general procedure B. The crude mixture was purified by column chromatography with petroleum ether as the eluent to give the product $\mathbf{4 b}$ as a colorless liquid (53.5 mg, 91\% yield). ${ }^{1} \mathbf{H}$ NMR (600 MHz, $\left.\mathrm{CDCl}_{3}\right) \delta 7.36$ (d, $\left.J=8.4 \mathrm{~Hz}, 2 \mathrm{H}\right), 7.26$ (d, $J=$ $8.4 \mathrm{~Hz}, 2 \mathrm{H}), 5.24(\mathrm{dd}, J=26.4,4.2 \mathrm{~Hz}, 1 \mathrm{H}), 1.31(\mathrm{~s}, 9 \mathrm{H}) .{ }^{13} \mathbf{C} \mathbf{N M R}\left(151 \mathrm{MHz}, \mathrm{CDCl}_{3}\right) \delta 156.2$ $(\mathrm{dd}, J=297.5,288.4 \mathrm{~Hz}), 150.1(\mathrm{t}, J=3.0 \mathrm{~Hz}), 127.4(\mathrm{t}, J=6.5 \mathrm{~Hz}), 127.3(\mathrm{dd}, J=6.0,3.5 \mathrm{~Hz})$, 125.6, $81.8(\mathrm{dd}, J=28.7,13.6 \mathrm{~Hz}), 34.5,31.2 .{ }^{19} \mathbf{F}$ NMR $\left(470 \mathrm{MHz}, \mathrm{CDCl}_{3}\right) \delta-83.05(\mathrm{dd}, J=33.4$, 26.4 Hz), -85.05 (dd, $J=33.4,3.8 \mathrm{~Hz}$ ). The spectral data is consistent with the reported literature. ${ }^{9}$

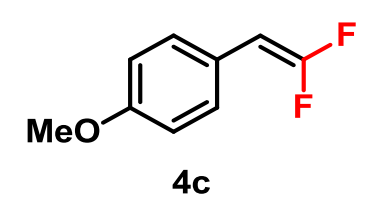

This compound was prepared according to the general procedure B. The crude mixture was purified by column chromatography with petroleum ether as the eluent to give the product $\mathbf{4 c}$ as a colorless liquid (45.9 mg, 90\% yield). ${ }^{1} \mathbf{H}$ NMR (600 MHz, $\left.\mathrm{CDCl}_{3}\right) \delta 7.25(\mathrm{~d}, J=8.4 \mathrm{~Hz}, 2 \mathrm{H}), 6.87(\mathrm{~d}, J=$ $8.4 \mathrm{~Hz}, 2 \mathrm{H}), 5.20(\mathrm{dd}, J=26.4,3.6 \mathrm{~Hz}, 1 \mathrm{H}), 3.79(\mathrm{~s}, 3 \mathrm{H}) .{ }^{13} \mathbf{C} \mathbf{N M R}\left(151 \mathrm{MHz}, \mathrm{CDCl}_{3}\right) \delta 158.5(\mathrm{t}$, $J=3.0 \mathrm{~Hz}), 155.8(\mathrm{dd}, J=296.0,286.9 \mathrm{~Hz}), 128.8(\mathrm{dd}, J=6.0,3.0 \mathrm{~Hz}), 122.7(\mathrm{t}, J=6.0 \mathrm{~Hz}), 114.1$, $81.5(\mathrm{dd}, J=28.7,13.6 \mathrm{~Hz}), 55.2 .{ }^{19}$ F NMR $\left(470 \mathrm{MHz}, \mathrm{CDCl}_{3}\right) \delta-84.65(\mathrm{dd}, J=36.9,26.4 \mathrm{~Hz}),-$ $86.46(\mathrm{dd}, J=36.9,3.0 \mathrm{~Hz})$. The spectral data is consistent with the reported literature. ${ }^{4}$ 


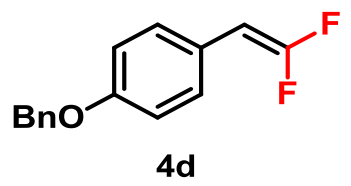

This compound was prepared according to the general procedure B. The crude mixture was purified by column chromatography with petroleum ether as the eluent to give the product $\mathbf{4 d}$ as a colorless liquid (65.4 mg, 94\% yield). ${ }^{1} \mathbf{H}$ NMR (600 MHz, $\left.\mathrm{CDCl}_{3}\right) \delta 7.42(\mathrm{~d}, J=7.2 \mathrm{~Hz}, 2 \mathrm{H}), 7.38$ (t, $J=$ $7.8 \mathrm{~Hz}, 2 \mathrm{H}), 7.32(\mathrm{t}, J=7.8 \mathrm{~Hz}, 1 \mathrm{H}), 7.24(\mathrm{~d}, J=8.4,2 \mathrm{H}), 6.93(\mathrm{~d}, J=8.4,2 \mathrm{H}), 5.19$ (dd, $J=26.4$, $1.8 \mathrm{~Hz}, 1 \mathrm{H}), 5.05(\mathrm{~s}, 2 \mathrm{H}) .{ }^{13} \mathrm{C} \mathbf{N M R}\left(151 \mathrm{MHz}, \mathrm{CDCl}_{3}\right) \delta 157.8,155.8(\mathrm{dd}, J=296.0,286.9 \mathrm{~Hz})$, 136.9, $128.8(\mathrm{dd}, J=6.0,3.0 \mathrm{~Hz}), 128.6,128.0,127.4,123.0(\mathrm{t}, J=6.4 \mathrm{~Hz}), 115.1,81.5(\mathrm{dd}, J=$ 28.7, 13.6 Hz), 70.0. ${ }^{19} \mathbf{F}$ NMR $\left(565 \mathrm{MHz}, \mathrm{CDCl}_{3}\right) \delta-84.52(\mathrm{dd}, J=36.6,26.4 \mathrm{~Hz}),-86.30(\mathrm{dd}, J=$ 36.6, $3.0 \mathrm{~Hz}$ ). The spectral data is consistent with the reported literature. ${ }^{8}$

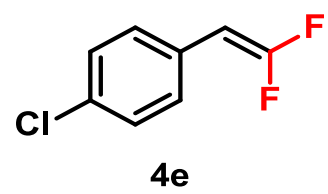

This compound was prepared according to the general procedure B. The crude mixture was purified by column chromatography with petroleum ether as the eluent to give the product $\mathbf{4 e}$ as a colorless liquid (41.8 mg, 80\% yield). ${ }^{1} \mathbf{H}$ NMR $\left(600 \mathrm{MHz}, \mathrm{CDCl}_{3}\right) \delta 7.29(\mathrm{~d}, J=8.4 \mathrm{~Hz}, 2 \mathrm{H}), 7.24(\mathrm{~d}, J=$ $8.4 \mathrm{~Hz}, 2 \mathrm{H}), 5.23(\mathrm{dd}, J=25.8,3.6 \mathrm{~Hz}, 1 \mathrm{H}) .{ }^{13} \mathbf{C ~ N M R}\left(151 \mathrm{MHz}, \mathrm{CDCl}_{3}\right) \delta 156.3(\mathrm{dd}, J=298.5$, $288.5 \mathrm{~Hz}), 132.8(\mathrm{t}, J=3.0 \mathrm{~Hz}), 128.87,128.85,128.80(\mathrm{dd}, J=6.8,3.0 \mathrm{~Hz}), 81.42(\mathrm{dd}, J=30.0$, 13.6 Hz). ${ }^{19} \mathbf{F}$ NMR $\left(565 \mathrm{MHz}, \mathrm{CDCl}_{3}\right) \delta-81.69(\mathrm{dd}, J=30.0,25.8 \mathrm{~Hz}),-83.46(\mathrm{dd}, J=30.0,4.0$ $\mathrm{Hz})$. The spectral data is consistent with the reported literature. ${ }^{4}$<smiles>FC(F)=Cc1ccc(I)cc1</smiles>

This compound was prepared according to the general procedure B. The crude mixture was purified by column chromatography with petroleum ether as the eluent to give the product $\mathbf{4 f}$ as a colorless liquid (64.6 mg, 81\% yield). ${ }^{1} \mathbf{H}$ NMR $\left(600 \mathrm{MHz}, \mathrm{CDCl}_{3}\right) \delta 7.64(\mathrm{~d}, J=8.4 \mathrm{~Hz}, 2 \mathrm{H}), 7.05(\mathrm{~d}, J=$ $8.4 \mathrm{~Hz}, 2 \mathrm{H}), 5.19(\mathrm{dd}, J=25.8,3.6 \mathrm{~Hz}, 1 \mathrm{H}) .{ }^{13} \mathrm{C}$ NMR $\left(151 \mathrm{MHz}, \mathrm{CDCl}_{3}\right) \delta 156.4(\mathrm{dd}, J=299.0$, $288.4 \mathrm{~Hz}$ ), 137.8, 129.9 (t, $J=6.0 \mathrm{~Hz}), 129.3$ (dd, $J=6.0,3.5 \mathrm{~Hz}), 92.2,81.6(\mathrm{dd}, J=30.2,13.6$ Hz). ${ }^{19}$ F NMR (470 MHz, $\left.\mathrm{CDCl}_{3}\right) \delta-80.78(\mathrm{dd}, J=28.2,25.8 \mathrm{~Hz}),-82.70(\mathrm{dd}, J=28.2,2.4 \mathrm{~Hz})$. The spectral data is consistent with the reported literature. ${ }^{9}$ 


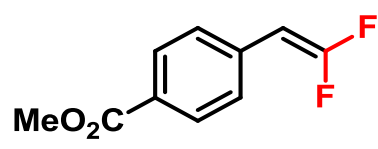

$4 \mathrm{~g}$

This compound was prepared according to the general procedure B. The crude mixture was purified by column chromatography with petroleum ether as the eluent to give the product $\mathbf{4 g}$ as a colorless liquid (47.5 mg, 80\% yield). ${ }^{1} \mathbf{H}$ NMR $\left(600 \mathrm{MHz}, \mathrm{CDCl}_{3}\right) \delta 8.00(\mathrm{~d}, J=8.4 \mathrm{~Hz}, 2 \mathrm{H}), 7.39(\mathrm{~d}, J=$ $8.4 \mathrm{~Hz}, 2 \mathrm{H}), 5.33(\mathrm{dd}, J=25.4,3.6 \mathrm{~Hz}, 1 \mathrm{H}), 3.91(\mathrm{~s}, 3 \mathrm{H}) .{ }^{13} \mathrm{C} \mathrm{NMR}\left(151 \mathrm{MHz}, \mathrm{CDCl}_{3}\right) \delta 166.7$, $156.8(\mathrm{dd}, J=300.5,291.4 \mathrm{~Hz}), 135.2(\mathrm{t}, J=6.5 \mathrm{~Hz}), 129.9,128.6,127.4(\mathrm{dd}, J=6.0,3.0 \mathrm{~Hz}), 82.0$ $(\mathrm{dd}, J=30.2,13.6 \mathrm{~Hz}), 52.1{ }^{19} \mathbf{F}$ NMR $\left(565 \mathrm{MHz}, \mathrm{CDCl}_{3}\right) \delta-79.24(\mathrm{t}, J=25.4 \mathrm{~Hz}),-81.16(\mathrm{dd}, J=$ 24.3, 4.0 Hz). The spectral data is consistent with the reported literature. ${ }^{8}$<smiles>CC(C)c1ccccc1C=C(F)F</smiles>

This compound was prepared according to the general procedure B. The crude mixture was purified by column chromatography with petroleum ether as the eluent to give the product $\mathbf{4 h}$ as a colorless liquid (44.8 mg, $82 \%$ yield). ${ }^{1} \mathbf{H}$ NMR $\left(600 \mathrm{MHz}, \mathrm{CDCl}_{3}\right) \delta 7.36(\mathrm{~d}, J=7.8 \mathrm{~Hz}, 1 \mathrm{H}), 7.29(\mathrm{~d}, J=$ $7.2 \mathrm{~Hz}, 1 \mathrm{H}), 7.27-7.23(\mathrm{~m}, 1 \mathrm{H}), 7.17(\mathrm{td}, J=7.8,1.2 \mathrm{~Hz}, 1 \mathrm{H}), 5.46(\mathrm{dd}, J=24.6,3.6 \mathrm{~Hz}, 1 \mathrm{H}), 3.11-$ $3.03(\mathrm{~m}, 1 \mathrm{H}), 1.22(\mathrm{~d}, J=6.6 \mathrm{~Hz}, 6 \mathrm{H}) .{ }^{13} \mathrm{C} \mathbf{N M R}\left(151 \mathrm{MHz}, \mathrm{CDCl}_{3}\right) \delta 156.3(\mathrm{dd}, J=294.5,285.0$ Hz), $146.6(\mathrm{~d}, J=4.5 \mathrm{~Hz}), 128.9(\mathrm{dd}, J=6.0,1.5 \mathrm{~Hz}), 127.8,127.5(\mathrm{dd}, J=7.6,4.5 \mathrm{~Hz}), 125.8$, $125.1,79.1(\mathrm{dd}, J=28.7,15.1 \mathrm{~Hz}), 29.8,23.3 .{ }^{19} \mathbf{F} \mathbf{~ N M R}\left(565 \mathrm{MHz}, \mathrm{CDCl}_{3}\right) \delta-84.56(\mathrm{dd}, J=32.8$, $4.0 \mathrm{~Hz}$ ), -86.39 (dd, $J=32.8,24.6 \mathrm{~Hz}$ ). IR (film): 2921, 1632, 1386, 1139, $676 \mathrm{~cm}^{-1}$. MS (EI, m/z): $182\left(\mathrm{M}^{+}, 100\right), 167(43)$.<smiles>FC(F)=Cc1ccccc1-c1ccccc1</smiles>

This compound was prepared according to the general procedure B. The crude mixture was purified by column chromatography with petroleum ether as the eluent to give the product $\mathbf{4 i}$ as a colorless liquid (54.4 mg, 84\% yield). ${ }^{1} \mathbf{H}$ NMR $\left(600 \mathrm{MHz}, \mathrm{CDCl}_{3}\right) \delta 7.60(\mathrm{~d}, J=7.8 \mathrm{~Hz}, 1 \mathrm{H}), 7.43-7.39(\mathrm{~m}$, 2H), 7.37-7.34 (m, 2H), 7.33-7.28 (m, 4H), 5.21 (dd, $J=26.0,4.2 \mathrm{~Hz}, 1 \mathrm{H}) .{ }^{13} \mathbf{C}$ NMR $(151 \mathrm{MHz}$, 
$\left.\mathrm{CDCl}_{3}\right) \delta 156.3(\mathrm{dd}, J=297.5,286.9 \mathrm{~Hz}), 141.3(\mathrm{dd}, J=1.0,4.5 \mathrm{~Hz}), 140.8,130.2,129.6,128.3$, $128.2(\mathrm{dd}, J=9.1,1.5 \mathrm{~Hz}), 128.0(\mathrm{t}, J=6.5 \mathrm{~Hz}), 127.6,127.3,127.2,80.6(\mathrm{dd}, J=30.2,12.1 \mathrm{~Hz})$. ${ }^{19}$ F NMR $\left(565 \mathrm{MHz}, \mathrm{CDCl}_{3}\right) \delta-83.11(\mathrm{dd}, J=32.8,4.2 \mathrm{~Hz}),-84.92(\mathrm{dd}, J=32.8,26.0 \mathrm{~Hz})$. The spectral data is consistent with the reported literature. ${ }^{6}$<smiles>FC(F)=Cc1ccccc1OCc1ccccc1</smiles>

This compound was prepared according to the general procedure B. The crude mixture was purified by column chromatography with petroleum ether as the eluent to give the product $\mathbf{4} \mathbf{j}$ as a colorless liquid (48.7 mg, 70\% yield). ${ }^{1} \mathbf{H}$ NMR $\left(600 \mathrm{MHz}, \mathrm{CDCl}_{3}\right) \delta 7.50(\mathrm{~d}, J=7.8 \mathrm{~Hz}, 1 \mathrm{H}), 7.43-7.38(\mathrm{~m}$, 4H), 7.35-7.33 (m, 1H), 7.22-7.17 (m, 1H), $6.97(\mathrm{t}, J=7.5 \mathrm{~Hz}, 1 \mathrm{H}), 6.93(\mathrm{~d}, J=8.4 \mathrm{~Hz}, 1 \mathrm{H}), 5.72$ $(\mathrm{dd}, J=27.1,4.2 \mathrm{~Hz}, 1 \mathrm{H}), 5.08(\mathrm{~s}, 2 \mathrm{H}) .{ }^{13} \mathbf{C} \mathbf{~ N M R}\left(151 \mathrm{MHz}, \mathrm{CDCl}_{3}\right) \delta 156.3(\mathrm{dd}, J=297.5,286.9$ Hz), $155.3(\mathrm{dd}, J=4.5,1.5 \mathrm{~Hz}), 136.8,128.6,128.4(\mathrm{dd}, \mathrm{J}=9.1,1.5 \mathrm{~Hz}), 128.1,128.0,127.3,121.0$, $119.6(\mathrm{t}, J=6.0 \mathrm{~Hz}), 112.1,76.3(\mathrm{dd}, J=31.7,12.1 \mathrm{~Hz}), 70.4 .{ }^{19} \mathbf{F}$ NMR $\left(470 \mathrm{MHz}, \mathrm{CDCl}_{3}\right) \delta-$ $83.22(\mathrm{dd}, J=31.5,3.8 \mathrm{~Hz}),-83.64(\mathrm{dd}, J=31.5,27.1 \mathrm{~Hz})$. The spectral data is consistent with the reported literature. ${ }^{4}$

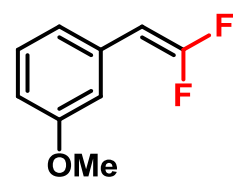

4k

This compound was prepared according to the general procedure B. The crude mixture was purified by column chromatography with petroleum ether as the eluent to give the product $\mathbf{4 k}$ as a colorless liquid (35.7 mg, 70\% yield). ${ }^{1} \mathbf{H}$ NMR $\left(600 \mathrm{MHz}, \mathrm{CDCl}_{3}\right) \delta 7.24(\mathrm{t}, J=7.8 \mathrm{~Hz}, 1 \mathrm{H}), 6.91(\mathrm{~d}, J=$ $7.8 \mathrm{~Hz}, 1 \mathrm{H}), 6.88(\mathrm{t}, J=2.0 \mathrm{~Hz}, 1 \mathrm{H}), 6.78(\mathrm{dd}, J=8.4,2.4 \mathrm{~Hz}, 1 \mathrm{H}), 5.24(\mathrm{dd}, J=25.8,3.6 \mathrm{~Hz}, 1 \mathrm{H})$, $3.79(\mathrm{~s}, 3 \mathrm{H}) .{ }^{13} \mathrm{C}$ NMR $\left(151 \mathrm{MHz}, \mathrm{CDCl}_{3}\right) \delta 159.7,156.3(\mathrm{dd}, J=290.0,288.4 \mathrm{~Hz}), 131.6(\mathrm{t}, J=$ $6.0 \mathrm{~Hz}), 129.6,120.2(\mathrm{dd}, J=6.0,3.0 \mathrm{~Hz}), 113.2(\mathrm{dd}, J=6.5,3.0 \mathrm{~Hz}), 112.6,82.2(\mathrm{dd}, J=28.7$, 13.6 Hz), 55.1. ${ }^{19} \mathbf{F}$ NMR $\left(565 \mathrm{MHz}, \mathrm{CDCl}_{3}\right) \delta-81.69(\mathrm{dd}, J=30.5,25.8 \mathrm{~Hz}),-83.97(\mathrm{dd}, J=30.5$, 4.0 Hz). The spectral data is consistent with the reported literature. ${ }^{10}$ 


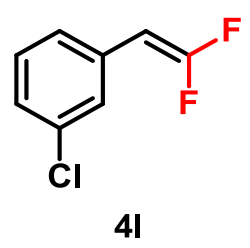

This compound was prepared according to the general procedure B. The crude mixture was purified by column chromatography with petroleum ether as the eluent to give the product $\mathbf{4 I}$ as a colorless liquid (39.7 mg, $76 \%$ yield). ${ }^{1} \mathbf{H}$ NMR (600 MHz, $\left.\mathrm{CDCl}_{3}\right) \delta 7.32(\mathrm{~s}, 1 \mathrm{H}), 7.25(\mathrm{~d}, J=7.8 \mathrm{~Hz}, 1 \mathrm{H})$, 7.22-7.17 (m, 2H), $5.23(\mathrm{dd}, J=25.8,3.6 \mathrm{~Hz}, 1 \mathrm{H}) .{ }^{13} \mathbf{C ~ N M R}\left(151 \mathrm{MHz}, \mathrm{CDCl}_{3}\right) \delta 156.6(\mathrm{dd}, J=$ 299.0, 289.6 Hz), 134.6, 132.2 (dd, $J=7.0,6.0 \mathrm{~Hz}), 129.9,127.5$ (dd, $J=6.0,3.0 \mathrm{~Hz}), 127.2$ (t, $J=$ $1.5 \mathrm{~Hz}), 125.7(\mathrm{dd}, J=6.5,3.5 \mathrm{~Hz}), 81.5(\mathrm{dd}, J=30.2,13.6 \mathrm{~Hz}) .{ }^{19} \mathbf{F} \mathbf{N M R}\left(565 \mathrm{MHz}, \mathrm{CDCl}_{3}\right) \delta-$ $80.61(\mathrm{dd}, J=27.7,25.8 \mathrm{~Hz}),-82.60(\mathrm{dd}, J=27.7,3.4 \mathrm{~Hz})$. The spectral data is consistent with the reported literature. ${ }^{11}$

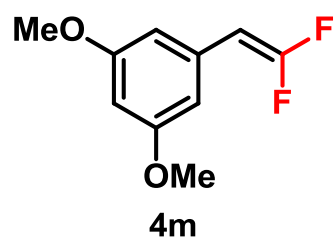

This compound was prepared according to the general procedure B. The crude mixture was purified by column chromatography with petroleum ether as the eluent to give the product $\mathbf{4 m}$ as a colorless liquid (52.3 mg, 87\% yield). ${ }^{1} \mathbf{H}$ NMR $\left(600 \mathrm{MHz}, \mathrm{CDCl}_{3}\right) \delta 6.48(\mathrm{~d}, J=2.4 \mathrm{~Hz}, 2 \mathrm{H}), 6.36(\mathrm{t}, J=$ $2.4 \mathrm{~Hz}, 1 \mathrm{H}), 5.20(\mathrm{dd}, J=26.0,3.6 \mathrm{~Hz}, 1 \mathrm{H}), 3.78(\mathrm{~s}, 6 \mathrm{H}) .{ }^{13} \mathrm{C} \mathbf{~ N M R}\left(151 \mathrm{MHz}, \mathrm{CDCl}_{3}\right) \delta 160.9$, $156.4(\mathrm{dd}, J=299.0,288.3 \mathrm{~Hz}), 132.1(\mathrm{dd}, J=7.6,6.0 \mathrm{~Hz}), 105.9(\mathrm{dd}, J=6.0,3.0 \mathrm{~Hz}), 99.3(\mathrm{~d}, J$ $=1.5 \mathrm{~Hz}), 82.4(\mathrm{dd}, J=28.7,12.1 \mathrm{~Hz}), 55.3 .{ }^{19} \mathbf{F} \mathbf{~ N M R}\left(565 \mathrm{MHz}, \mathrm{CDCl}_{3}\right) \delta-81.15(\mathrm{dd}, J=30.5$, $26.0 \mathrm{~Hz}$ ), -83.82 (dd, $J=30.5,4.0 \mathrm{~Hz}$ ). IR (film): 2937, 1731, 1595, 1460, 1427, 1208, 1157, 1065, 898, 850, $669 \mathrm{~cm}^{-1}$. MS (EI, m/z): $200\left(\mathrm{M}^{+}, 100\right), 140(37)$.<smiles>FC(F)=Cc1ccc(Cl)c(Cl)c1</smiles>

This compound was prepared according to the general procedure B. The crude mixture was purified by column chromatography with petroleum ether as the eluent to give the product $4 \mathrm{n}$ as a colorless liquid (48.4 mg, 78\% yield). ${ }^{1} \mathbf{H}$ NMR $\left(600 \mathrm{MHz}, \mathrm{CDCl}_{3}\right) \delta 7.40(\mathrm{~d}, J=2.4 \mathrm{~Hz}, 1 \mathrm{H}), 7.38(\mathrm{~d}, J=$ $8.4 \mathrm{~Hz}, 1 \mathrm{H}), 7.14(\mathrm{dd}, J=8.4,2.4 \mathrm{~Hz}, 1 \mathrm{H}), 5.21(\mathrm{dd}, J=25.8,3.6 \mathrm{~Hz}, 1 \mathrm{H}) .{ }^{13} \mathbf{C}$ NMR $(151 \mathrm{MHz}$, 
$\left.\mathrm{CDCl}_{3}\right) \delta 156.6(\mathrm{dd}, J=299.0,290.4 \mathrm{~Hz}), 132.9,131.0(\mathrm{t}, J=3.0 \mathrm{~Hz}), 130.6,130.4(\mathrm{dd}, J=7.2,6.6$ Hz), $129.2(\mathrm{dd}, J=7.6,4.5 \mathrm{~Hz}), 126.8(\mathrm{dd}, J=6.0,3.0 \mathrm{~Hz}), 80.9(\mathrm{dd}, J=30.2,13.6 \mathrm{~Hz}) .{ }^{19} \mathbf{F}$ NMR $\left(565 \mathrm{MHz}, \mathrm{CDCl}_{3}\right) \delta-80.06(\mathrm{t}, J=26.0 \mathrm{~Hz}),-81.85(\mathrm{dd}, J=26.0,4.0 \mathrm{~Hz})$. The spectral data is consistent with the reported literature. ${ }^{12}$

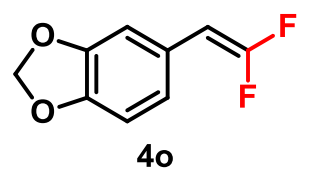

This compound was prepared according to the general procedure B. The crude mixture was purified by column chromatography with petroleum ether as the eluent to give the product $\mathbf{4 0}$ as a colorless liquid (48.0 mg, 87\% yield). ${ }^{1} \mathbf{H}$ NMR $\left(600 \mathrm{MHz}, \mathrm{CDCl}_{3}\right) \delta 6.87(\mathrm{~d}, J=1.8 \mathrm{~Hz}, 1 \mathrm{H}), 6.76(\mathrm{~d}, J=$ $7.8 \mathrm{~Hz}, 1 \mathrm{H}), 6.73(\mathrm{dd}, J=7.8,1.8 \mathrm{~Hz}, 1 \mathrm{H}) 5.94(\mathrm{~s}, 2 \mathrm{H}), 5.18(\mathrm{dd}, J=26.4,4.2 \mathrm{~Hz}, 1 \mathrm{H}) .{ }^{13} \mathbf{C}$ NMR $\left(151 \mathrm{MHz}, \mathrm{CDCl}_{3}\right) \delta 155.9(\mathrm{dd}, J=296.7,287.2 \mathrm{~Hz}), 148.0,146.6(\mathrm{t}, J=1.5 \mathrm{~Hz}), 124.1$ (t, $J=6.0$ Hz), $121.6(\mathrm{t}, J=4.5 \mathrm{~Hz}), 108.5,107.7(\mathrm{dd}, J=7.6,3.0 \mathrm{~Hz}), 101.1,82.0(\mathrm{dd}, J=28.7,13.6 \mathrm{~Hz}) .{ }^{19} \mathbf{F}$ NMR $\left(565 \mathrm{MHz}, \mathrm{CDCl}_{3}\right) \delta-83.90(\mathrm{dd}, J=36.2,26.4 \mathrm{~Hz}),-86.10(\mathrm{dd}, J=36.2,4.0 \mathrm{~Hz})$. The spectral data is consistent with the reported literature. ${ }^{8}$

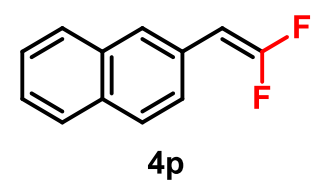

This compound was prepared according to the general procedure B. The crude mixture was purified by column chromatography with petroleum ether as the eluent to give the product $\mathbf{4 p}$ as a colorless liquid (38.2 mg, 67\% yield). ${ }^{1} \mathbf{H}$ NMR (600 MHz, $\left.\mathrm{CDCl}_{3}\right) \delta$ 7.79-7.73 (m, 3H), 7.71 (s, 1H), 7.46$7.41(\mathrm{~m}, 3 \mathrm{H}), 5.39(\mathrm{dd}, J=26.4,3.6 \mathrm{~Hz}, 1 \mathrm{H}) .{ }^{13} \mathrm{C} \mathbf{~ N M R}\left(151 \mathrm{MHz}, \mathrm{CDCl}_{3}\right) \delta 156.5(\mathrm{dd}, J=297.5$, $288.6 \mathrm{~Hz}), 133.4,132.3,128.3,127.8$ (t, $J=6.0 \mathrm{~Hz}), 127.8,127.6,126.6$ (t, $J=6.0 \mathrm{~Hz}), 126.4$, 126.0, $125.3(\mathrm{dd}, J=7.6,3.0 \mathrm{~Hz}), 82.4(\mathrm{dd}, J=28.7,12.1 \mathrm{~Hz}) .{ }^{19} \mathbf{F} \mathbf{N M R}\left(565 \mathrm{MHz}, \mathrm{CDCl}_{3}\right) \delta-$ $81.93(\mathrm{dd}, J=30.5,26.4 \mathrm{~Hz}),-83.63(\mathrm{dd}, J=30.5,4.0 \mathrm{~Hz})$. The spectral data is consistent with the reported literature. ${ }^{4}$<smiles>FC(F)=Cc1cccc2ccccc12</smiles> 
This compound was prepared according to the general procedure B. The crude mixture was purified by column chromatography with petroleum ether as the eluent to give the product $\mathbf{4 q}$ as a colorless liquid (45.1 mg, 79\% yield). ${ }^{1} \mathbf{H}$ NMR $\left(600 \mathrm{MHz}, \mathrm{CDCl}_{3}\right) \delta 7.94(\mathrm{~d}, J=8.4 \mathrm{~Hz}, 1 \mathrm{H}), 7.85$ (d, $J=$ $7.8 \mathrm{~Hz}, 1 \mathrm{H}), 7.78(\mathrm{~d}, J=8.4 \mathrm{~Hz}, 1 \mathrm{H}), 7.57(\mathrm{~d}, J=7.2 \mathrm{~Hz}, 1 \mathrm{H}), 7.58-7.45(\mathrm{~m}, 3 \mathrm{H}), 5.86(\mathrm{dd}, J=$ 24.0, $2.4 \mathrm{~Hz}, 1 \mathrm{H}) .{ }^{13} \mathbf{C}$ NMR $\left(151 \mathrm{MHz}, \mathrm{CDCl}_{3}\right) \delta 156.7$ (dd, $\left.J=296.0,288.4 \mathrm{~Hz}\right), 133.7,131.47$, 131.45, 128.7, 128.0, $126.5(\mathrm{dd}, J=7.6,3.0 \mathrm{~Hz}), 126.4,126.0,125.5,123.8,78.7(\mathrm{dd}, J=30.2,16.6$ Hz). ${ }^{19}$ F NMR $\left(565 \mathrm{MHz}, \mathrm{CDCl}_{3}\right) \delta-83.24(\mathrm{~d}, J=29.4 \mathrm{~Hz}),-85.01(\mathrm{dd}, J=29.4,24.0 \mathrm{~Hz})$. The spectral data is consistent with the reported literature. ${ }^{13}$

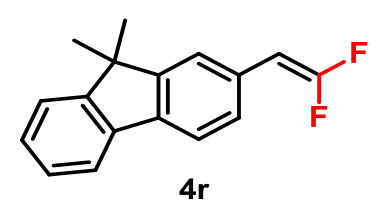

This compound was prepared according to the general procedure B. The crude mixture was purified by column chromatography with petroleum ether as the eluent to give the product $\mathbf{4 r}$ as a colorless liquid $\left(66.0 \mathrm{mg}, 86 \%\right.$ yield). ${ }^{1} \mathbf{H}$ NMR $\left(600 \mathrm{MHz}, \mathrm{CDCl}_{3}\right) \delta 7.68(\mathrm{~d}, J=7.2 \mathrm{~Hz}, 1 \mathrm{H}), 7.65(\mathrm{~d}, J=$ $7.8 \mathrm{~Hz}, 1 \mathrm{H}), 7.42-7.40(\mathrm{~m}, 1 \mathrm{H}), 7.38(\mathrm{~s}, 1 \mathrm{H}), 7.35-7.25(\mathrm{~m}, 3 \mathrm{H}), 5.33(\mathrm{dd}, J=26.4,3.6 \mathrm{~Hz}, 1 \mathrm{H})$, $1.47(\mathrm{~s}, 6 \mathrm{H}) .{ }^{13} \mathrm{C}$ NMR $\left(151 \mathrm{MHz}, \mathrm{CDCl}_{3}\right) \delta 156.3(\mathrm{dd}, J=297.5,288.4 \mathrm{~Hz}), 154.2,153.8,138.8$, $138.3(\mathrm{t}, J=1.5 \mathrm{~Hz}), 129.3(\mathrm{t}, J=6.0 \mathrm{~Hz}), 127.4,127.1,126.7(\mathrm{dd}, J=6.0,3.0 \mathrm{~Hz}), 122.7,121.8$ $(\mathrm{dd}, J=7.0,3.5 \mathrm{~Hz}), 120.3,120.1,82.7(\mathrm{dd}, J=28.7,13.6 \mathrm{~Hz}), 46.9,27.2 .{ }^{19} \mathbf{F}$ NMR $(565 \mathrm{MHz}$, $\left.\mathrm{CDCl}_{3}\right) \delta-82.19(\mathrm{dd}, J=32.2,26.4 \mathrm{~Hz}$ ), -84.55 (dd, $J=32.2,4.0 \mathrm{~Hz}$ ). IR (film): 2960, 17261223 , 1164, $736 \mathrm{~cm}^{-1}$. MS (EI, m/z): $256\left(\mathrm{M}^{+}, 73\right), 241(100)$.

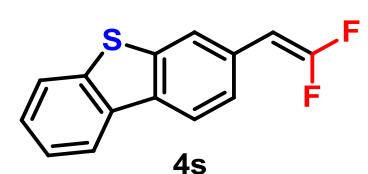

This compound was prepared according to the general procedure B. The crude mixture was purified by column chromatography with petroleum ether as the eluent to give the product $4 \mathbf{s}$ as a colorless liquid (41.4 mg, 56\% yield). ${ }^{1} \mathbf{H}$ NMR $\left(600 \mathrm{MHz}, \mathrm{CDCl}_{3}\right) \delta 8.12-8.08(\mathrm{~m}, 1 \mathrm{H}), 8.01(\mathrm{~d}, J=7.8 \mathrm{~Hz}$, 1H), 7.86-7.82 (m, 1H), 7.56 (d, $J=7.8 \mathrm{~Hz}, 1 \mathrm{H}), 7.47-7.41(\mathrm{~m}, 3 \mathrm{H}), 5.50(\mathrm{dd}, J=25.2,3.0 \mathrm{~Hz}, 1 \mathrm{H})$. ${ }^{13}$ C NMR $\left(151 \mathrm{MHz}, \mathrm{CDCl}_{3}\right) \delta 156.9(\mathrm{dd}, \mathrm{J}=300.5,291.4 \mathrm{~Hz}), 138.7,135.85,135.82,126.9,125.3$ $(\mathrm{dd}, J=9.1,1.5 \mathrm{~Hz}), 124.90,124.89(\mathrm{dd}, J=7.0,6.0 \mathrm{~Hz}), 124.6,122.7,121.8,120.3,79.9(\mathrm{dd}, J=$ $30.2,15.1 \mathrm{~Hz}) .{ }^{19} \mathbf{F}$ NMR $\left(565 \mathrm{MHz}, \mathrm{CDCl}_{3}\right) \delta-80.62(\mathrm{t}, J=25.0 \mathrm{~Hz}),-81.62(\mathrm{dd}, J=25.0,2.8 \mathrm{~Hz})$. 
IR (film): 2923, 1729, 1395, 1349, 1206, 955, 819, $751 \mathrm{~cm}^{-1} . \mathbf{M S}$ (EI, m/z): $246\left(\mathrm{M}^{+}, 100\right), 226$ (10), $195(13)$.

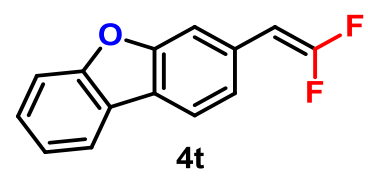

This compound was prepared according to the general procedure B. The crude mixture was purified by column chromatography with petroleum ether as the eluent to give the product $\mathbf{4 t}$ as a colorless liquid (43.5 mg, 63\% yield). ${ }^{1} \mathbf{H}$ NMR $\left(600 \mathrm{MHz}, \mathrm{CDCl}_{3}\right) \delta 7.94(\mathrm{~d}, J=7.8 \mathrm{~Hz}, 1 \mathrm{H}), 7.82(\mathrm{~d}, J=$ $7.8 \mathrm{~Hz}, 1 \mathrm{H}), 7.61-7.56(\mathrm{~m}, 2 \mathrm{H}), 7.47(\mathrm{t}, J=8.4 \mathrm{~Hz}, 1 \mathrm{H}), 7.37-7.31(\mathrm{~m}, 2 \mathrm{H}), 5.91(\mathrm{dd}, J=26.4,3.0$ $\mathrm{Hz}, 1 \mathrm{H}) .{ }^{13} \mathbf{C}$ NMR $\left(151 \mathrm{MHz}, \mathrm{CDCl}_{3}\right) \delta 158.8(\mathrm{dd}, J=299.0,289.9 \mathrm{~Hz}), 155.9,153.3(\mathrm{dd}, J=4.5$, $1.5 \mathrm{~Hz}), 127.4,125.2(\mathrm{dd}, J=10.6,3.0 \mathrm{~Hz}), 124.24,124.19,123.1,122.9,120.8,119.3,115.1(\mathrm{dd}$, $J=7.6,6.0 \mathrm{~Hz}), 111.7,75.8(\mathrm{dd}, J=33.2,15.1 \mathrm{~Hz}) .{ }^{19} \mathbf{F} \mathbf{N M R}\left(470 \mathrm{MHz}, \mathrm{CDCl}_{3}\right) \delta-80.33(\mathrm{t}, J=$ $26.4 \mathrm{~Hz}),-81.62(\mathrm{dd}, J=25.4,2.4 \mathrm{~Hz})$. The spectral data is consistent with the reported literature. ${ }^{8}$

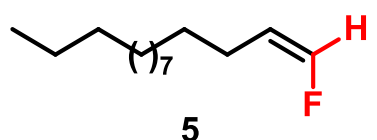

Colorless liquid (30.8 mg, 72\% yield). ${ }^{1} \mathbf{H}$ NMR $\left(600 \mathrm{MHz}, \mathrm{CDCl}_{3}\right) \delta 6.43(\mathrm{dd}, J=85.8,4.8 \mathrm{~Hz}$, 1H), 4.71 (dtd, $J=43.2,7.8,4.8 \mathrm{~Hz}, 1 \mathrm{H}), 2.12-2.08(\mathrm{~m}, 2 \mathrm{H}), 1.37-1.26(\mathrm{~m}, 20 \mathrm{H}), 0.88$ (t, $J=7.8$ $\mathrm{Hz}, 3 \mathrm{H}) .{ }^{13} \mathrm{C}$ NMR $\left(151 \mathrm{MHz}, \mathrm{CDCl}_{3}\right) \delta 147.5(\mathrm{~d}, J=255.2 \mathrm{~Hz}), 111.1(\mathrm{~d}, J=6.0 \mathrm{~Hz}), 32.0,29.73$, 29.70, 29.67, 29.6, 29.42, 29.38, $29.2(\mathrm{~d}, J=1.5 \mathrm{~Hz}), 29.1,22.71,22.66(\mathrm{~d}, J=6.0 \mathrm{~Hz}), 14.1 .{ }^{19} \mathbf{F}$ NMR (470 MHz, $\left.\mathrm{CDCl}_{3}\right) \delta-131.50$ (dd, $J=86.0,43.7 \mathrm{~Hz}$ ). IR (film): 2925, 2855, 1746, 1672, 1462, 1377, 1262, 1034, 752, $670 \mathrm{~cm}^{-1}$. MS (EI, m/z): 214 (100).

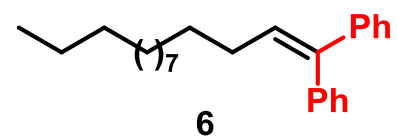

Colorless liquid (113.1 mg, 65\% yield). ${ }^{1} \mathbf{H}$ NMR $\left(600 \mathrm{MHz}, \mathrm{CDCl}_{3}\right) \delta 7.35(\mathrm{t}, J=7.2 \mathrm{~Hz}, 2 \mathrm{H})$, $7.29(\mathrm{t}, J=7.2 \mathrm{~Hz}, 1 \mathrm{H}), 7.26-7.20(\mathrm{~m}, 5 \mathrm{H}), 7.19-7.16(\mathrm{~d}, J=6.6 \mathrm{~Hz}, 2 \mathrm{H}), 6.08(\mathrm{t}, \mathrm{J}=7.2 \mathrm{~Hz}, 1 \mathrm{H})$, $2.10(\mathrm{q}, J=7.2 \mathrm{~Hz}, 2 \mathrm{H}), 1.45-1.23(\mathrm{~m}, 20 \mathrm{H}), 0.88(\mathrm{t}, J=6.6 \mathrm{~Hz}, 3 \mathrm{H}) .{ }^{13} \mathbf{C} \mathbf{N M R}\left(151 \mathrm{MHz}, \mathrm{CDCl}_{3}\right)$ $\delta 142.9,141.4,140.4,130.3,130.0,128.1(\mathrm{~d}, J=7.6 \mathrm{~Hz}), 127.2,126.7$ (d, $J=10.6 \mathrm{~Hz}), 31.9,30.0$, 29.8, 29.7 (d, $J=4.5 \mathrm{~Hz}$ ), 29.6, 29.5, 29.4, 29.3, 22.7, 14.1. IR (film): 2924, 2853, 1493, 1463, 1444, 760, $699 \mathrm{~cm}^{-1} . \mathbf{M S}(\mathrm{EI}, \mathrm{m} / \mathrm{z}): 348\left(\mathrm{M}^{+}, 12\right), 193(100)$. 


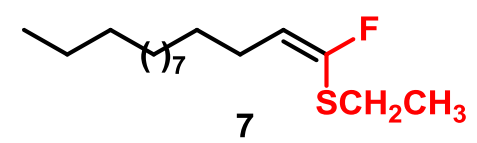

Colorless liquid (42.7 mg, 78\% yield). ${ }^{1} \mathbf{H}$ NMR $\left(600 \mathrm{MHz}, \mathrm{CDCl}_{3}\right) \delta 5.56-5.51(\mathrm{~m}, 1 \mathrm{H}), 2.73(\mathrm{q}, J$ $=7.2 \mathrm{~Hz}, 2 \mathrm{H}), 2.11(\mathrm{q}, J=7.2 \mathrm{~Hz}, 2 \mathrm{H}), 1.32(\mathrm{~m}, 23 \mathrm{H}), 0.88(\mathrm{t}, J=7.0 \mathrm{~Hz}, 3 \mathrm{H}) .{ }^{13} \mathbf{C}$ NMR $(151$ $\left.\mathrm{MHz}, \mathrm{CDCl}_{3}\right) \delta 154.1,152.2,116.3(\mathrm{~d}, J=24.2 \mathrm{~Hz}), 31.9,29.70,29.67,29.64,29.59,29.5(\mathrm{~d}, J=$ $1.5 \mathrm{~Hz}), 29.41,29.35,29.1,27.3(\mathrm{~d}, J=3.0 \mathrm{~Hz}), 26.1,22.7,15.0,14.1 .{ }^{19} \mathbf{F}$ NMR (470 MHz, $\left.\mathrm{CDCl}_{3}\right)$ $\delta-88.87$ (d, $J=11.8 \mathrm{~Hz}$ ). IR (film): 2925, 2854, 1746, 1647, 1462, 1380, 1258, $1081 \mathrm{~cm}^{-1}$. MS (EI, m/z): $274\left(\mathrm{M}^{+}, 34\right), 245$ (100), 225 (40), 212(71).

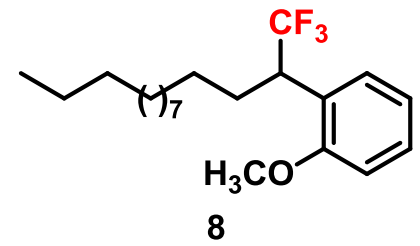

Colorless liquid $\left(76.7 \mathrm{mg}, 83 \%\right.$ yield). ${ }^{1} \mathbf{H}$ NMR $\left(600 \mathrm{MHz}, \mathrm{CDCl}_{3}\right) \delta 7.31(\mathrm{~d}, J=7.8 \mathrm{~Hz}, 1 \mathrm{H})$, 7.28-7.25 (m, 1H), $6.97(\mathrm{t}, J=7.2 \mathrm{~Hz}, 1 \mathrm{H}), 6.90(\mathrm{~d}, J=7.8 \mathrm{~Hz}, 1 \mathrm{H}), 4.07-4.00(\mathrm{~m}, 1 \mathrm{H}), 3.82(\mathrm{~s}$, 3H), $1.99-1.77(\mathrm{~m}, 2 \mathrm{H}), 1.30-1.15(\mathrm{~m}, 20 \mathrm{H}), 0.88(\mathrm{t}, J=7.2 \mathrm{~Hz}, 3 \mathrm{H}) .{ }^{13} \mathbf{C} \mathbf{N M R}\left(151 \mathrm{MHz}, \mathrm{CDCl}_{3}\right)$ $\delta 158.0,128.8,128.2,127.4(\mathrm{q}, J=279.4 \mathrm{~Hz}), 123.8,120.8,110.9,55.7,40.1(\mathrm{q}, J=27 . \mathrm{Hz}), 32.0$, $29.67,29.66,29.60,29.57,29.4,29.3,29.2,28.7(\mathrm{~d}, J=1.5 \mathrm{~Hz}), 26.5,22.7,14.1 .{ }^{19} \mathbf{F}$ NMR $(470$ $\left.\mathrm{MHz}, \mathrm{CDCl}_{3}\right) \delta-69.47$ (d, $J=9.4 \mathrm{~Hz}$ ). IR (film): 2926, 2855, 1495, 1464, 1247, 1159, 1132, 1107, $753 \mathrm{~cm}^{-1}$. MS (EI, m/z): $358\left(\mathrm{M}^{+}, 97\right), 189(33), 121$ (100). 


\section{References}

1. Wang, Y.; Guan, R.; Sivaguru, P.; Cong, X.; Bi, X. Org Lett 2019, 21, 4035.

2 Li, Z.; Wang, Z.; Zhu, L.; Tan, X.; Li, C. J Am Chem Soc. 2014, 136, 16439.

3. Fuchibe, K.; Oki, R.; Hatta, H.; Ichikawa, J. Chem Eur J. 2018, 24, 17932.

4. Hu, J.; Han, X.; Yuan, Y.; Shi, Z. Angew. Chem. Int. Ed. 2017, 56, 13342

5. Prakash, G. K. S.; Hu, J.; Wang, Y.; Olah, G. A. Angew. Chem. Int. Ed. 2004, 116, 5315.

6. Yokota, M.; Fujita, D.; Ichikawa, J. Org. Lett. 2007, 9, 4639.

7. Argintaru, O. A.; Ryu, D.; Aron, I.; Molander, G. A. Angew. Chem. Int. Ed. 2013, 52, 13656.

8. Gøgsig, T. M.; Søbjerg, L. S.; Lindhardt, A. T.; Jensen, K. L.; Skrydstrup, T. J. Org. Chem. 2008, $73,3404$.

9. Nenajdenko, V. G.; Varseev, G. N.; Korotchenko, V. N.; Shastin, A. V.; Balenkova, E. S. J. Fluorine Chem. 2003, 124, 115.

10. Nguyen, B. V.; Burton, D. J. J. Org. Chem. 1997, 62, 7758.

11. Thornbury, R. T.; Toste, F. D. Angew. Chem. Int. Ed. 2016, 55, 11629.

12. Wu, G.; Deng, Y.; Wu, C.; Wang, X.; Zhang, Y.; Wang, J. Eur. J. Org. Chem. 2014, 2014, 4477.

13. Nowak, I.; Robins, M. J. Org. Lett. 2005, 7, 721. 


\section{Copies of NMR spectra}

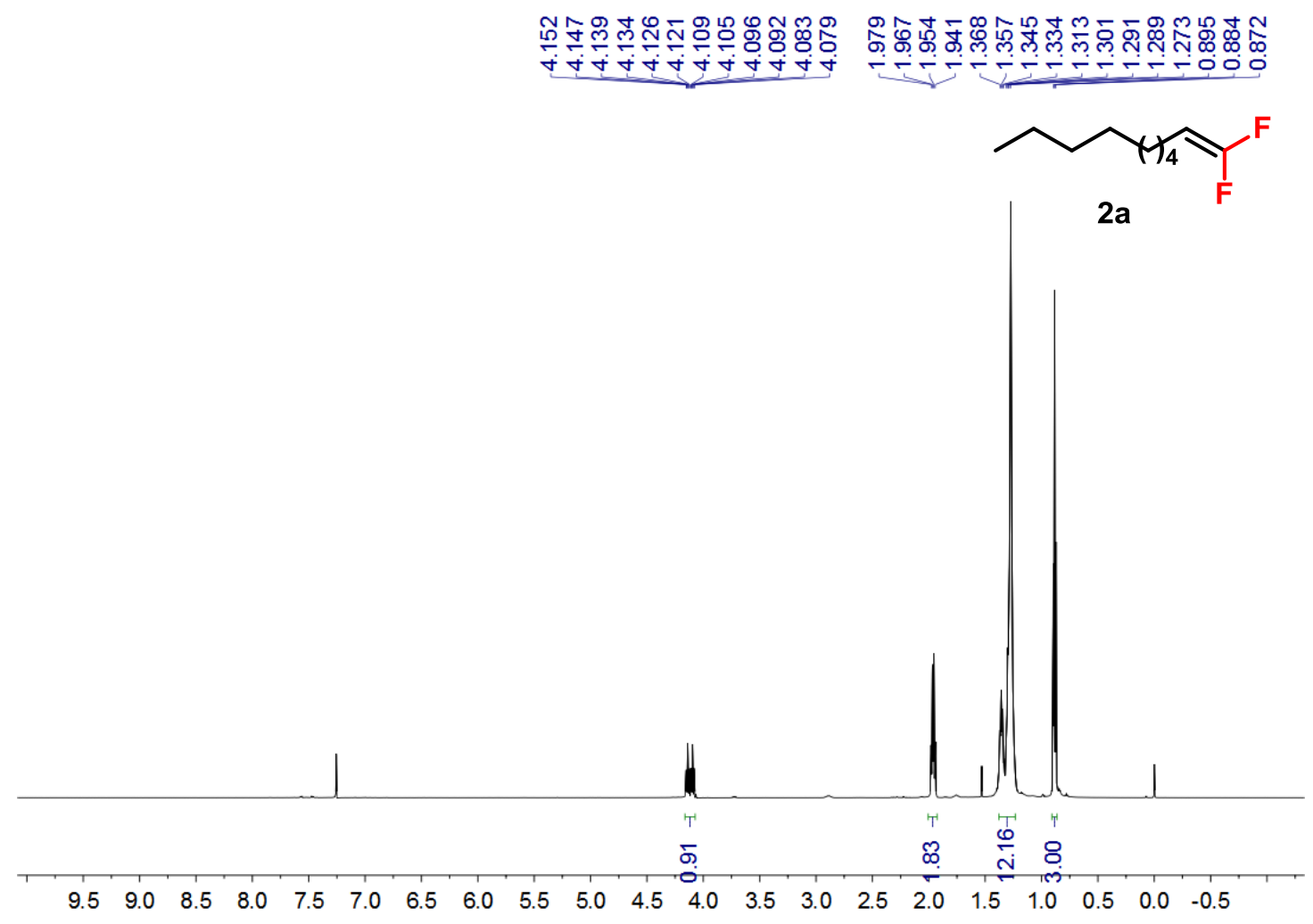

กิกับิก

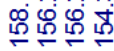

응요

$\sqrt{\infty}$

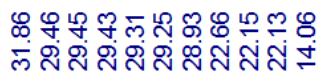

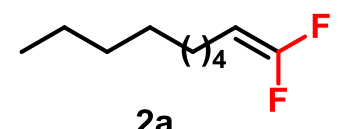

2a

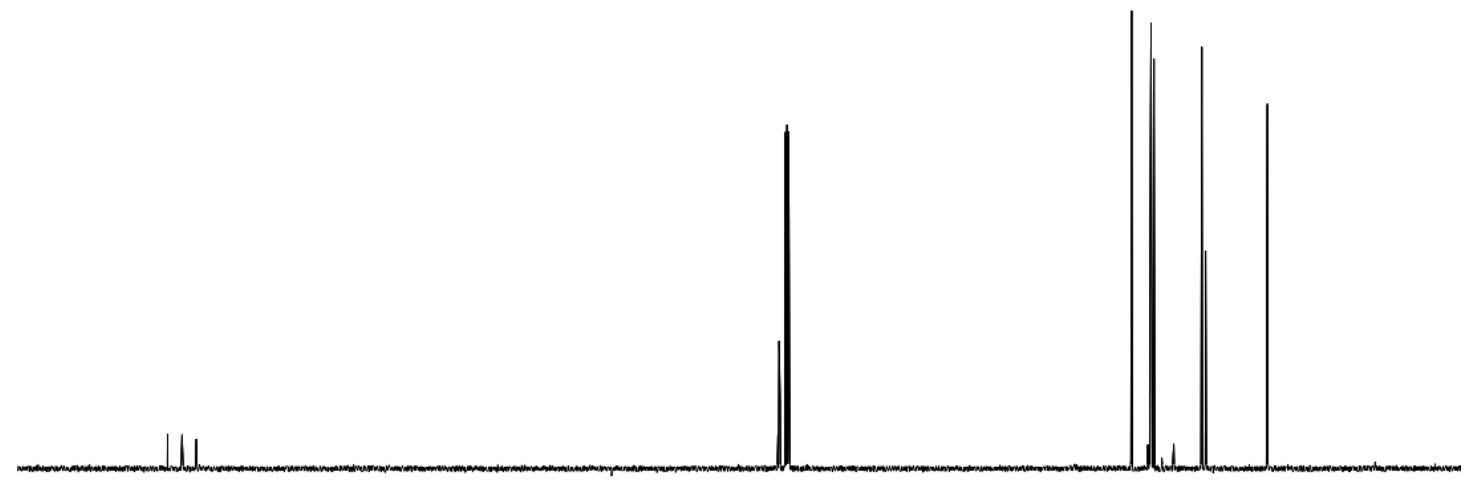

$\begin{array}{lllllllllllllllllll}170 & 160 & 150 & 140 & 130 & 120 & 110 & 100 & 90 & 80 & 70 & 60 & 50 & 40 & 30 & 20 & 10 & 0 & -11\end{array}$ 

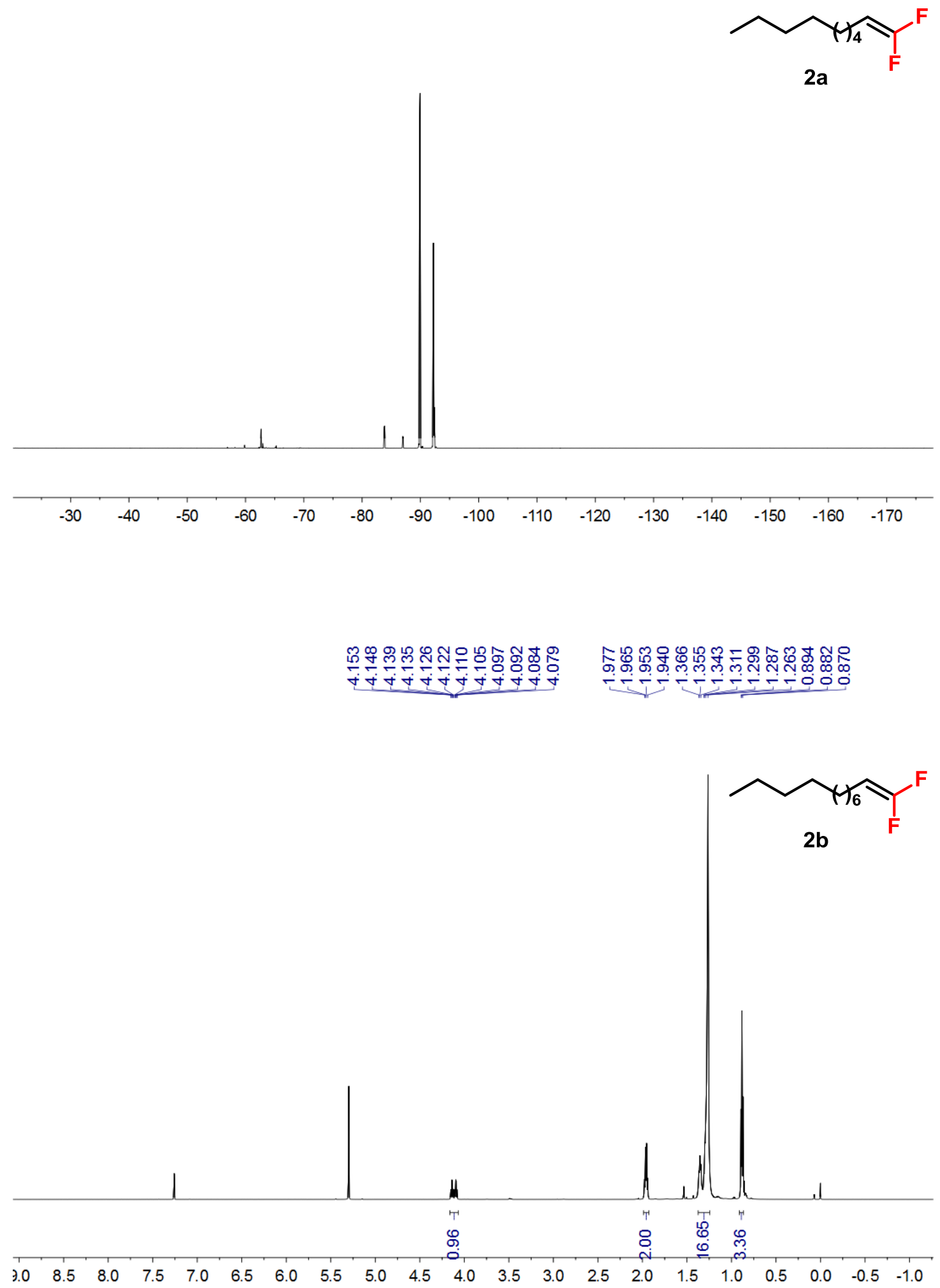


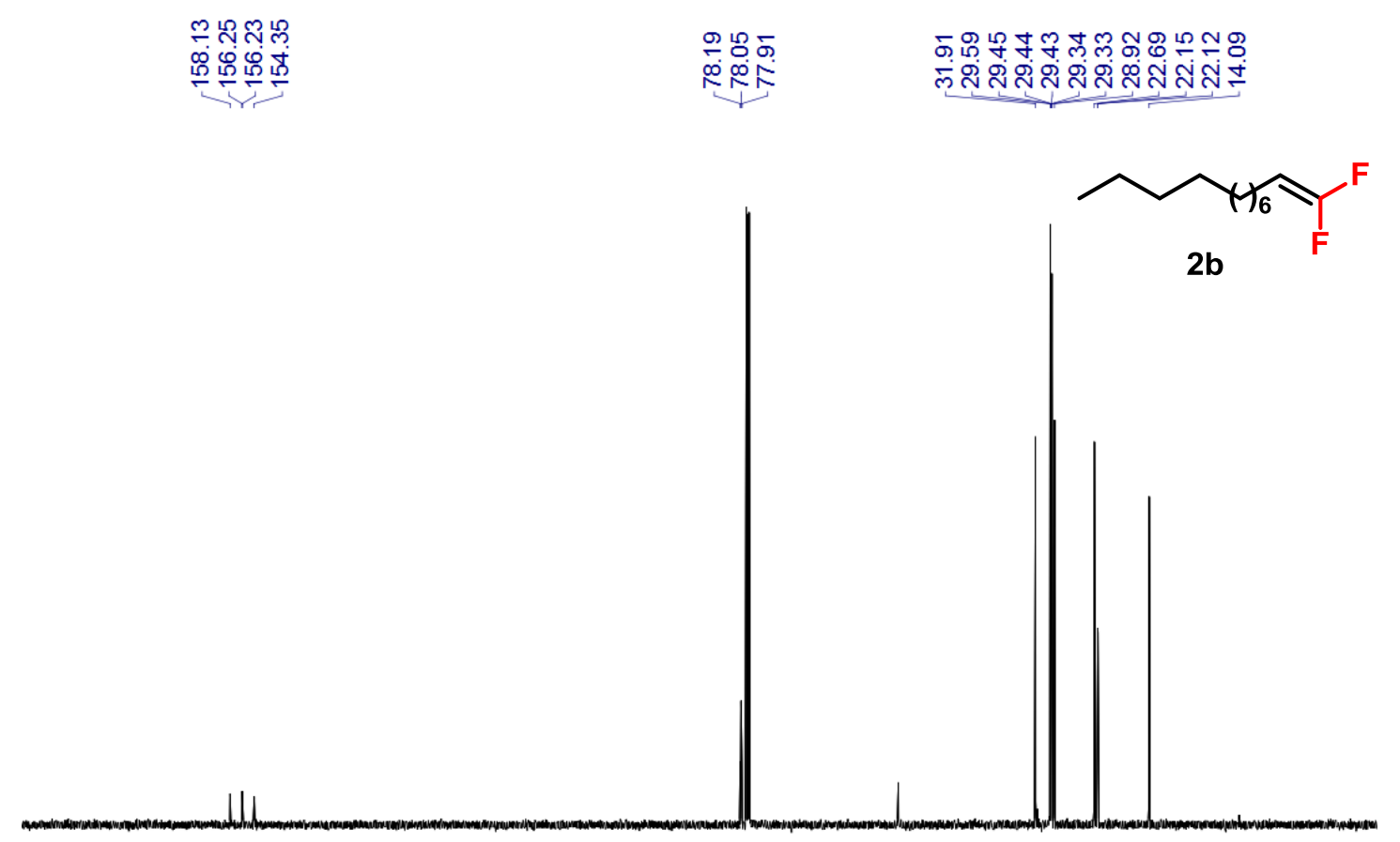

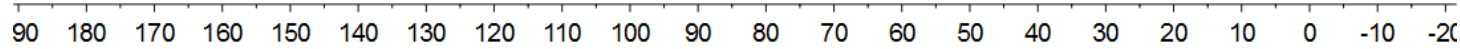

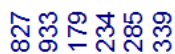

ळळ

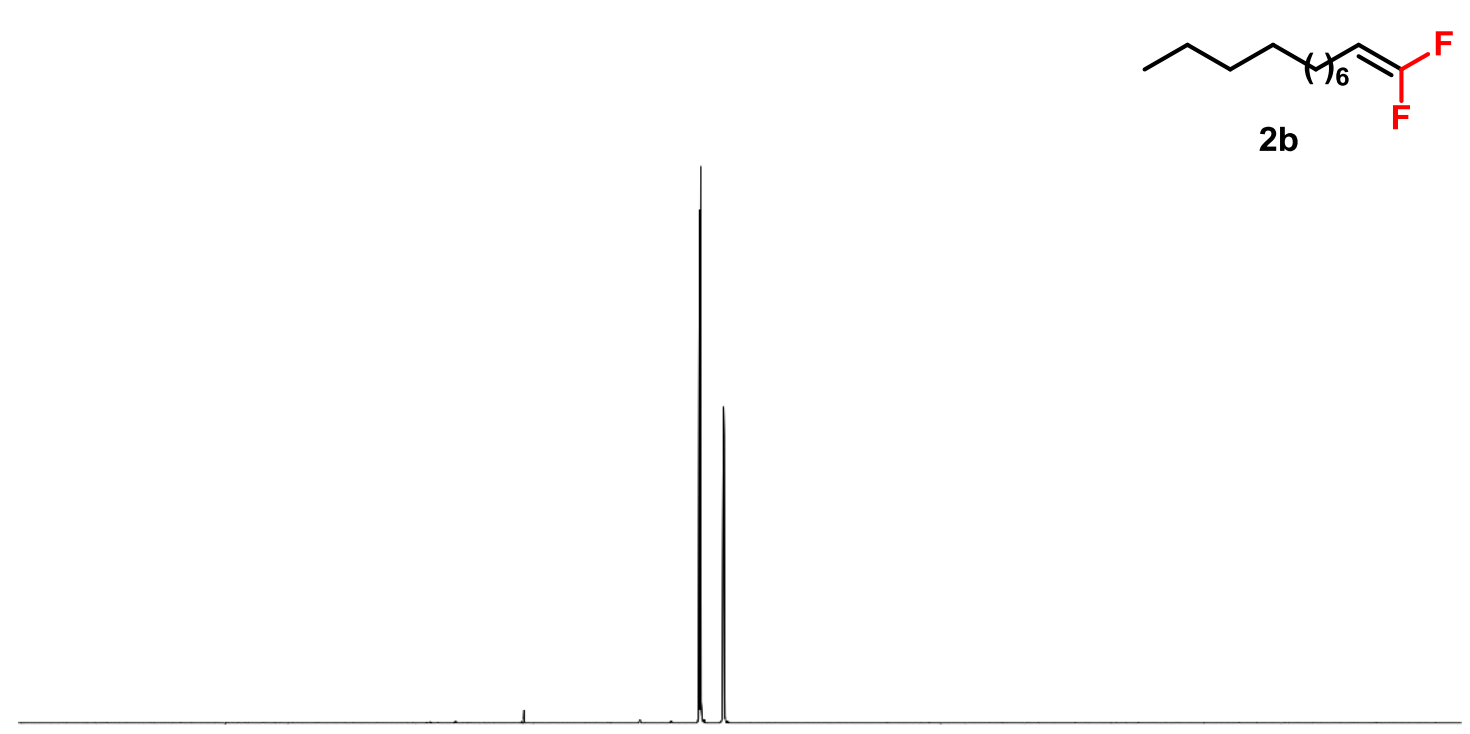

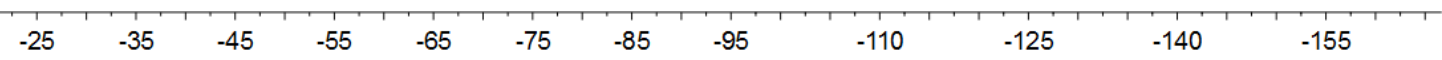



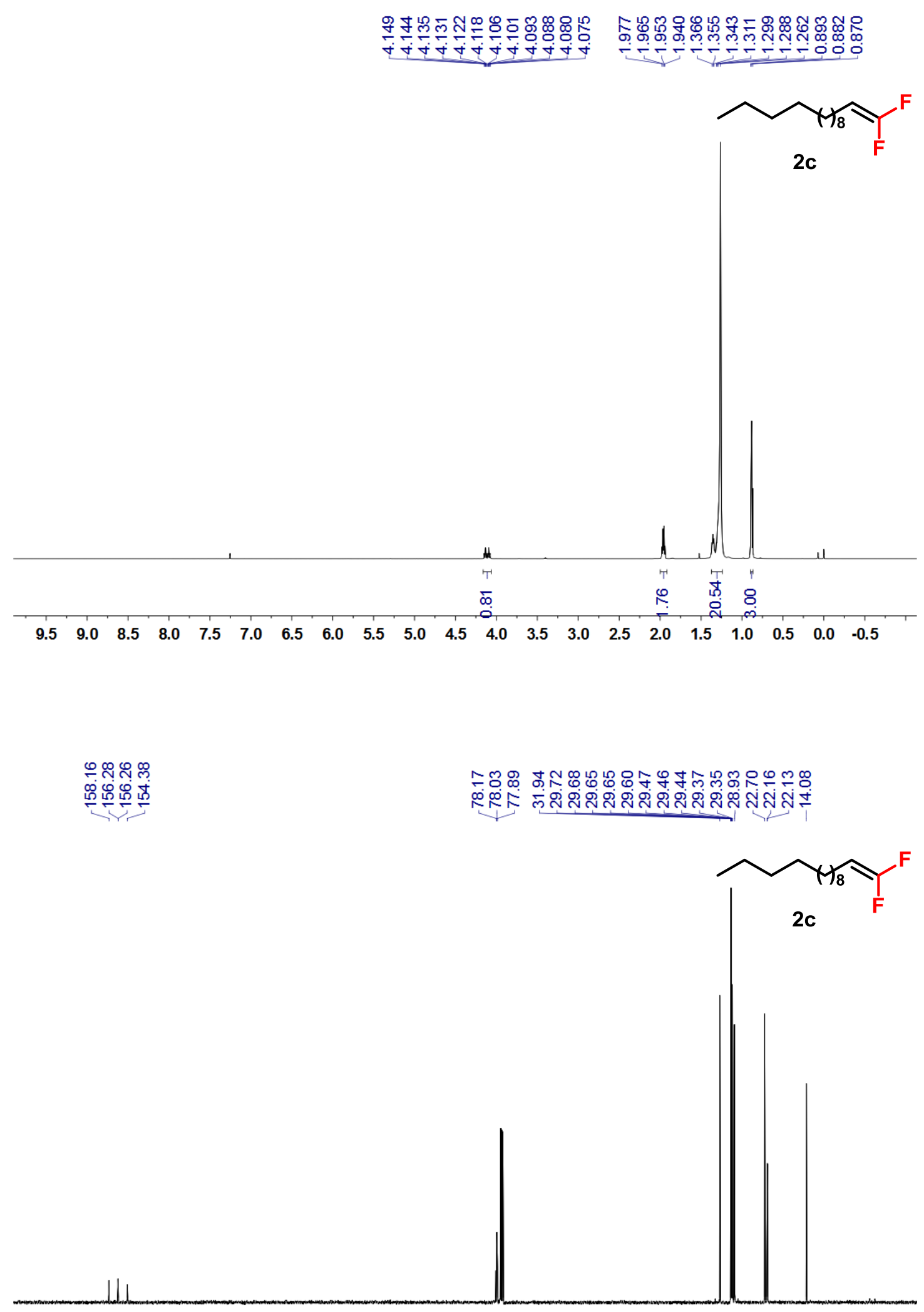

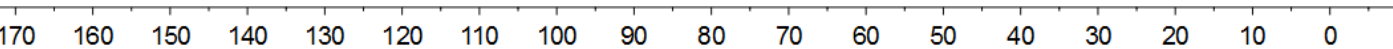




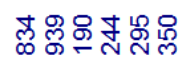

ळ
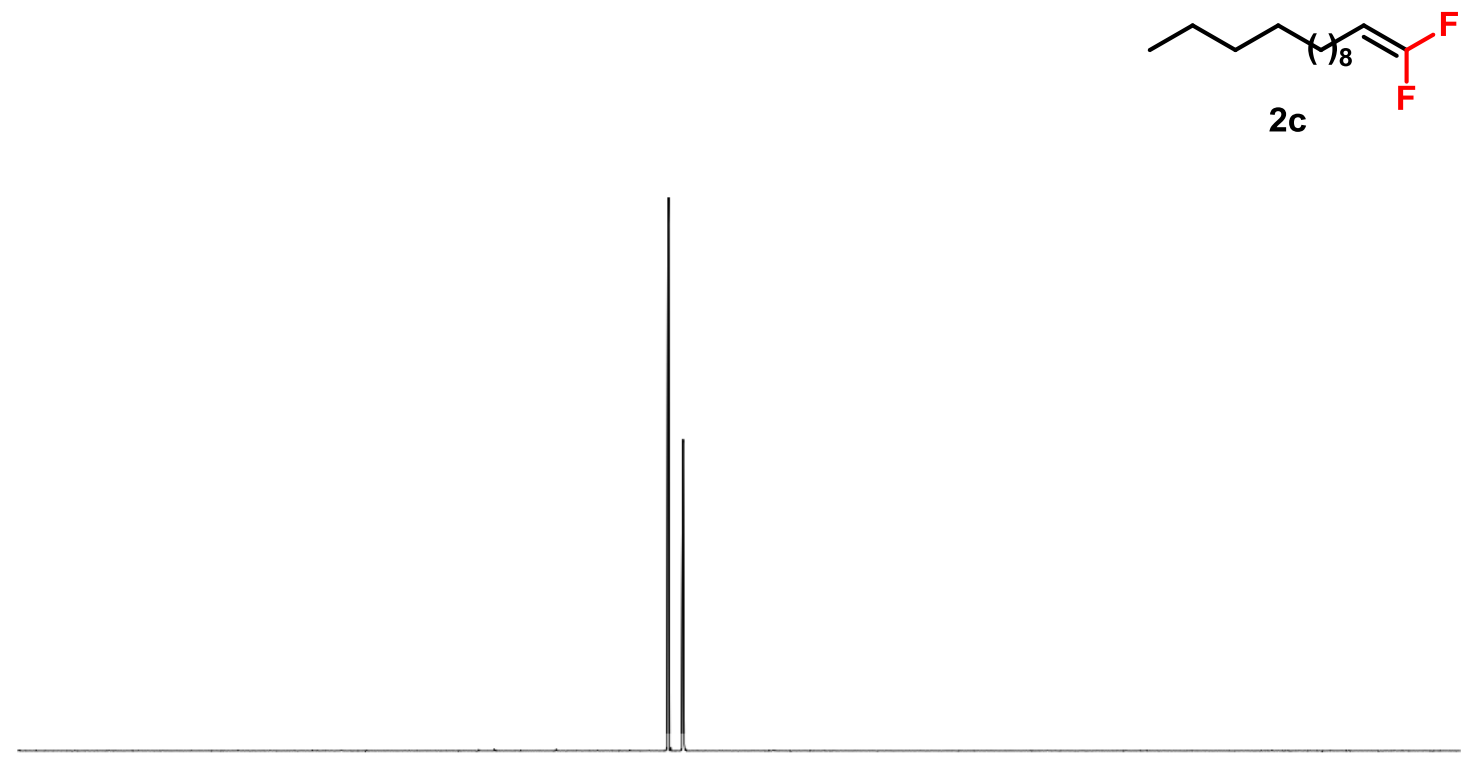

\begin{tabular}{llllllllllllllllll}
\hline 10 & 0 & -10 & -20 & -30 & -40 & -50 & -60 & -70 & -80 & -90 & -100 & -120 & -140 & -160 & -180 & -200
\end{tabular}

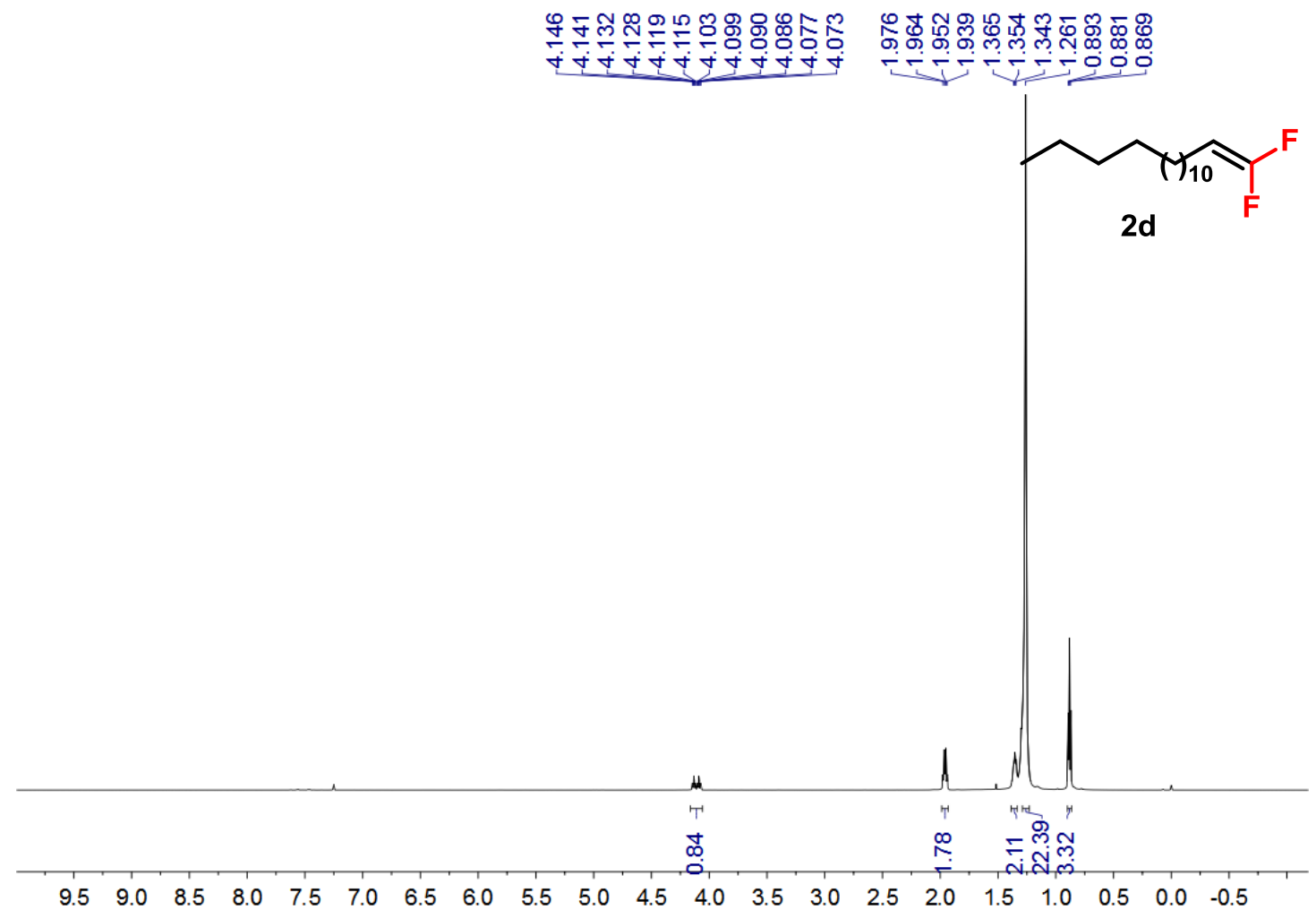




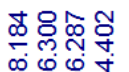

兽品品安

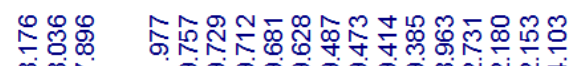

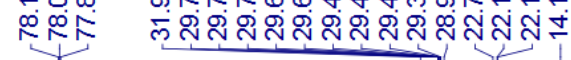

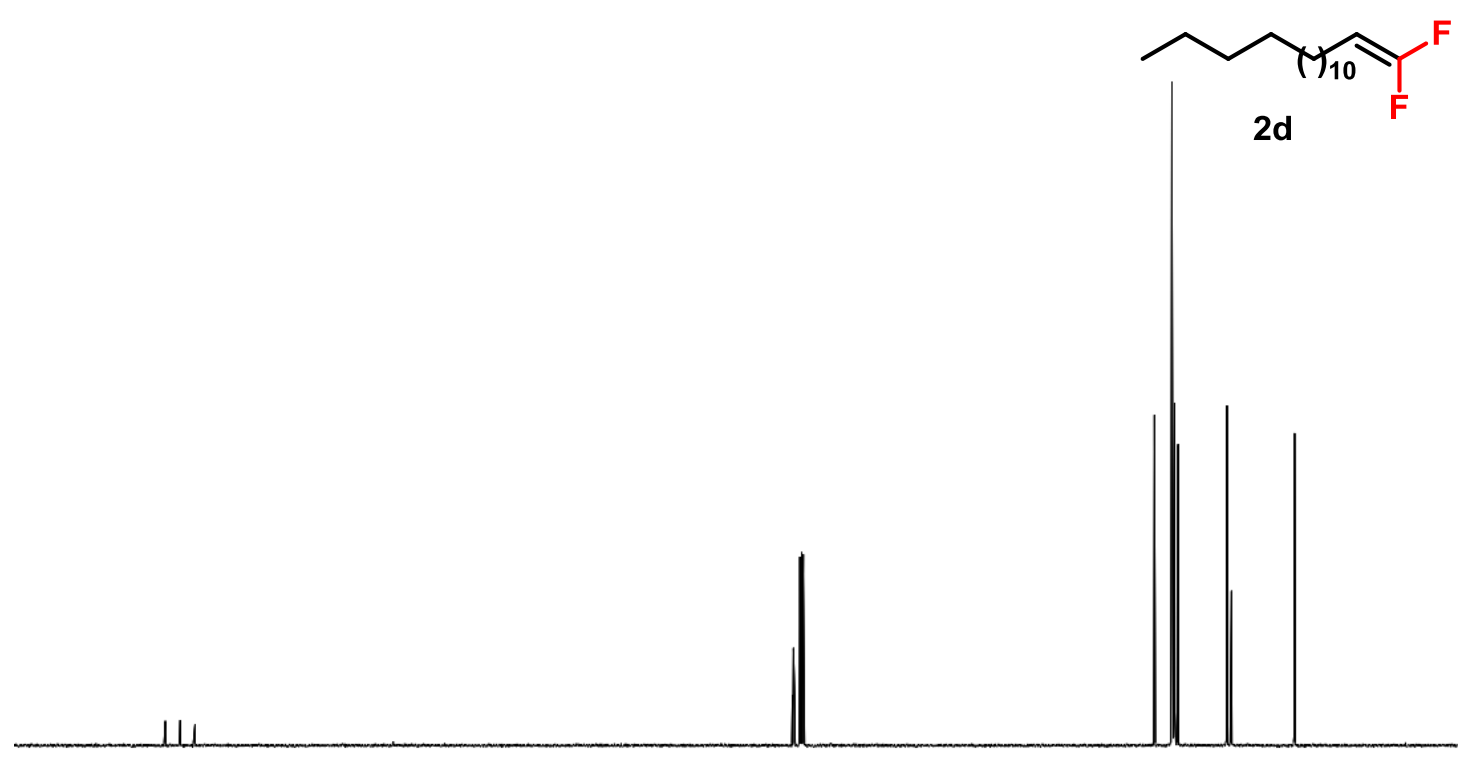

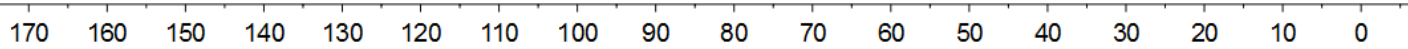

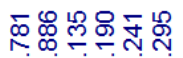

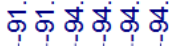

11
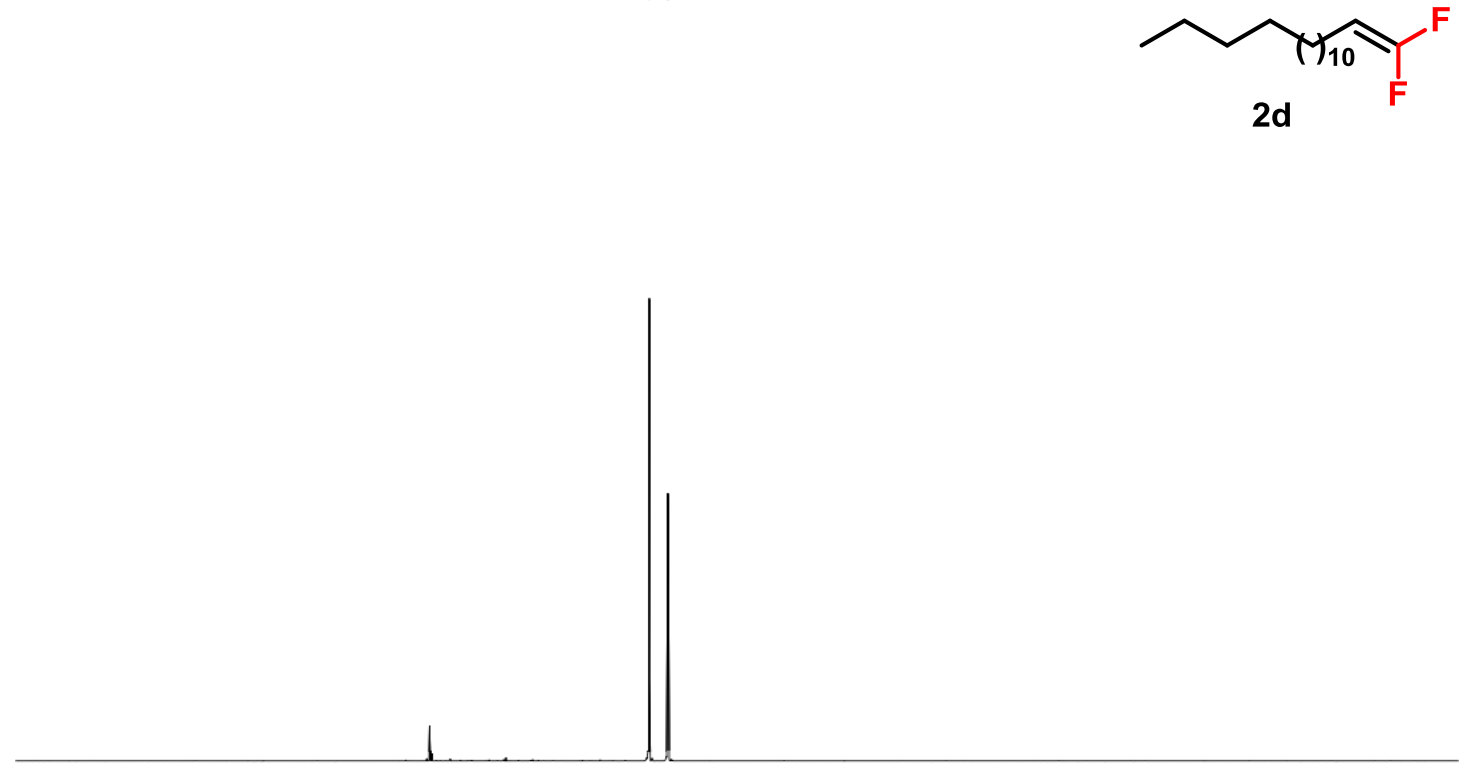

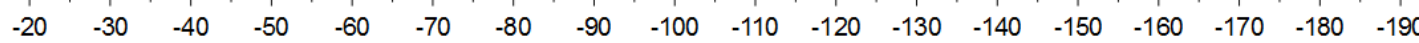



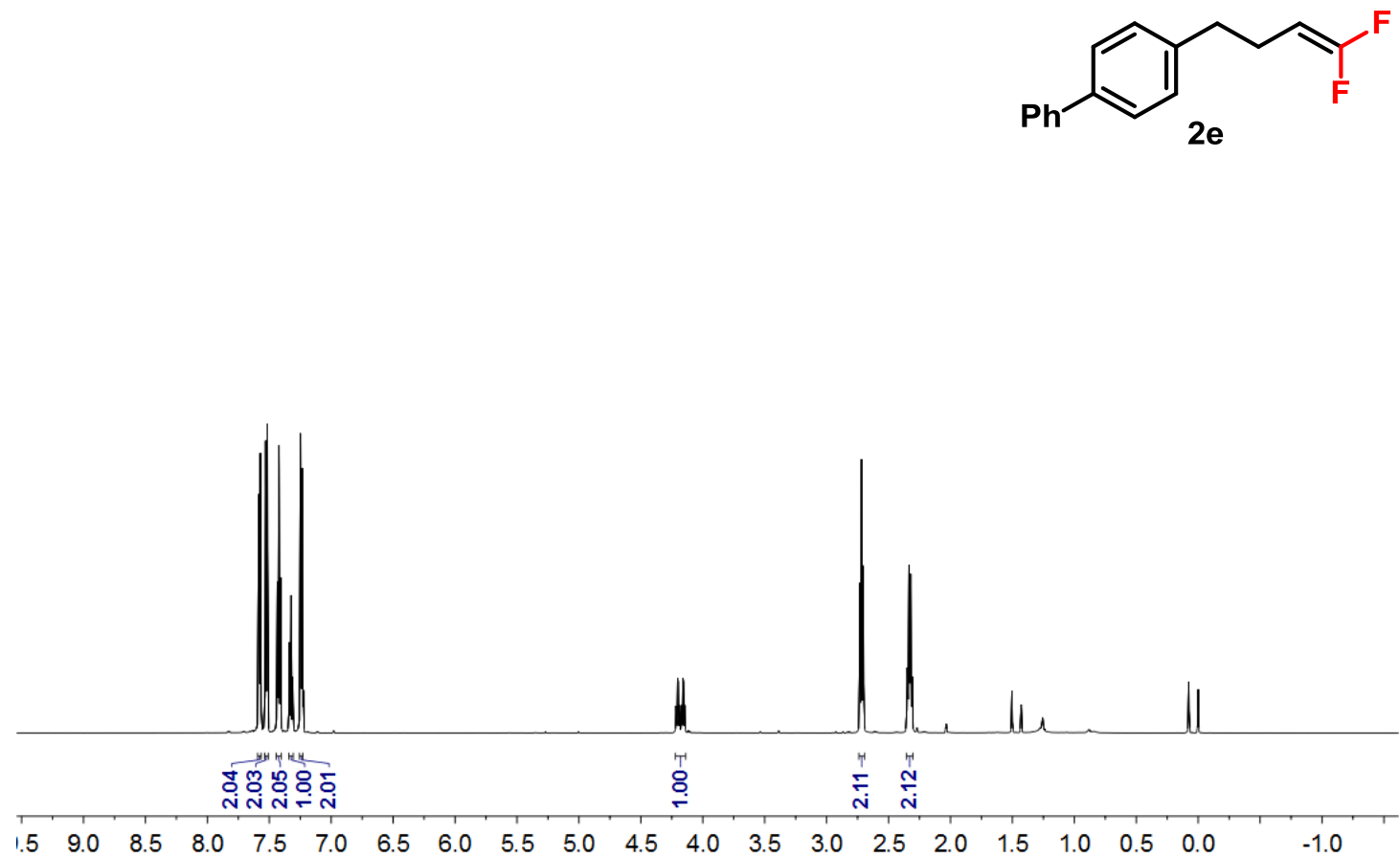

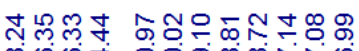

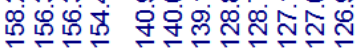

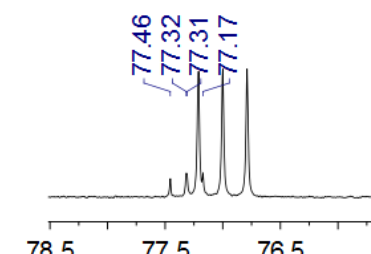

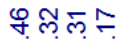

FRRE

ำกำ 8 ติ

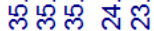

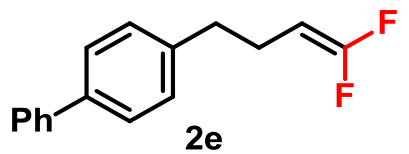

$\begin{array}{lllllllllllllllllllll}190 & 180 & 170 & 160 & 150 & 140 & 130 & 120 & 110 & 100 & 90 & 80 & 70 & 60 & 50 & 40 & 30 & 20 & 10 & 0 & -10\end{array}$ 


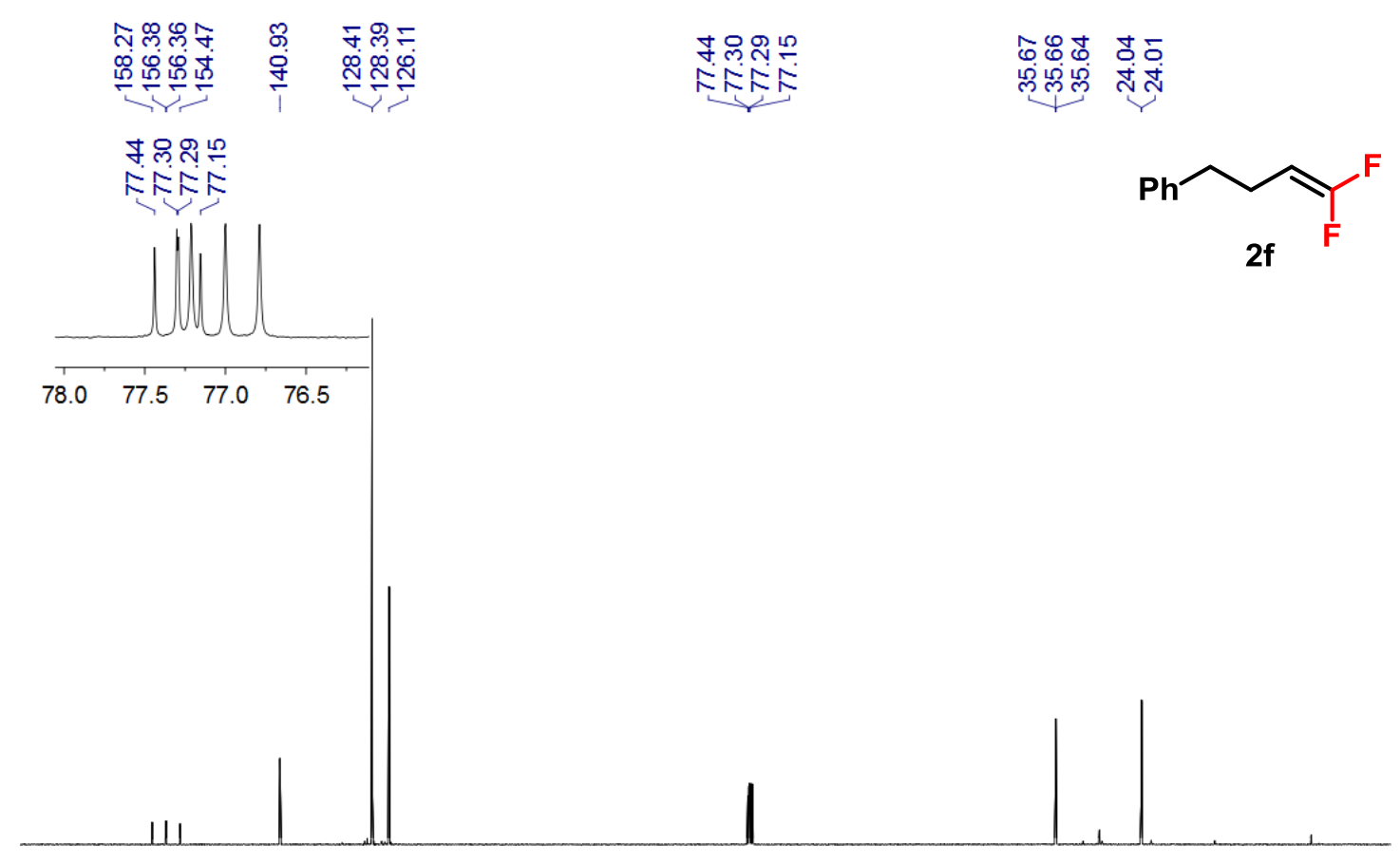

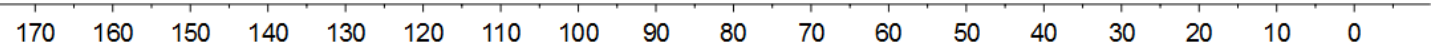

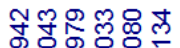

œ्ं किंक

ㄴ,

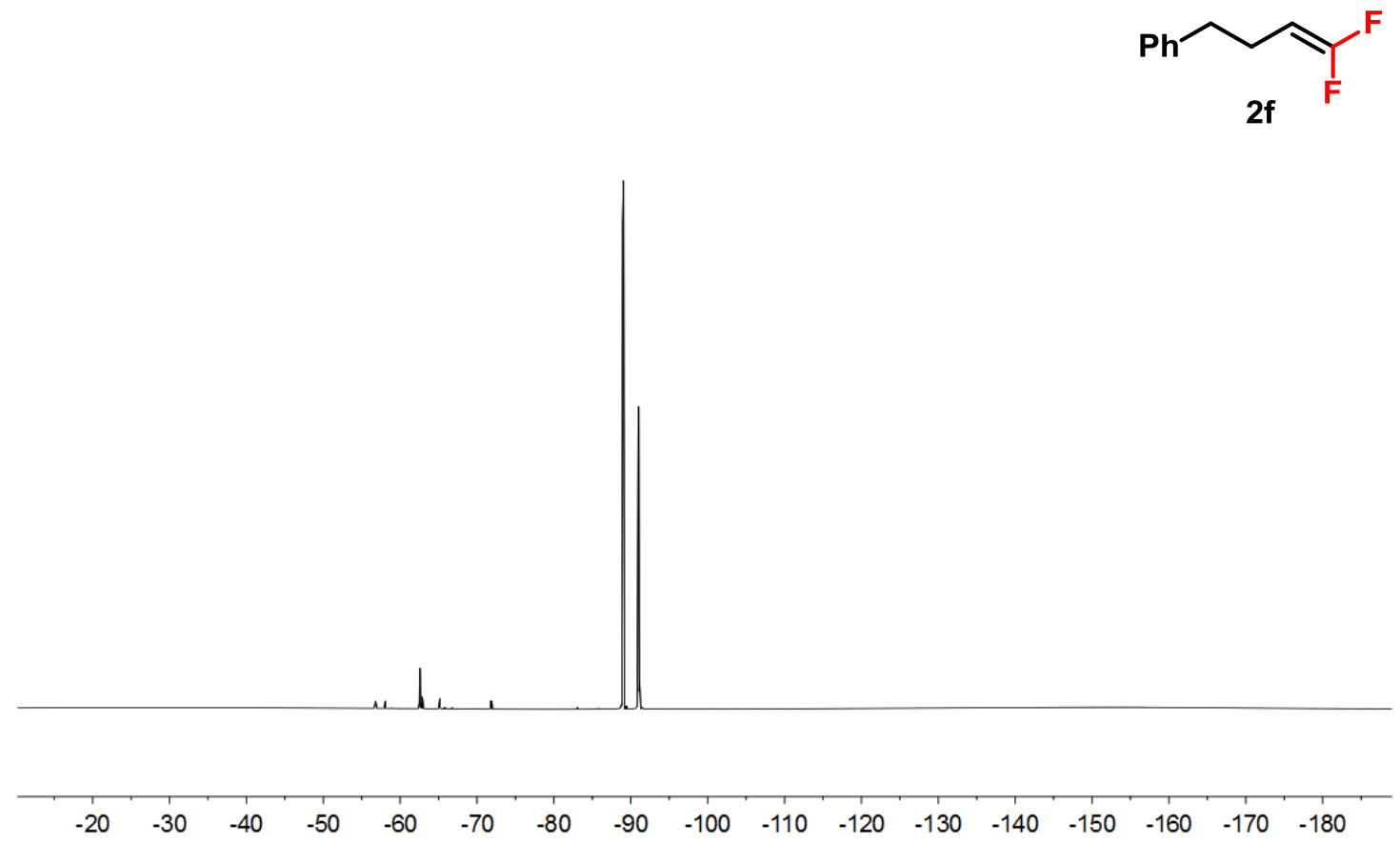


$\mathrm{Ph}$<smiles>FC(F)=CCCCc1ccccc1</smiles>

$2 \mathrm{~g}$
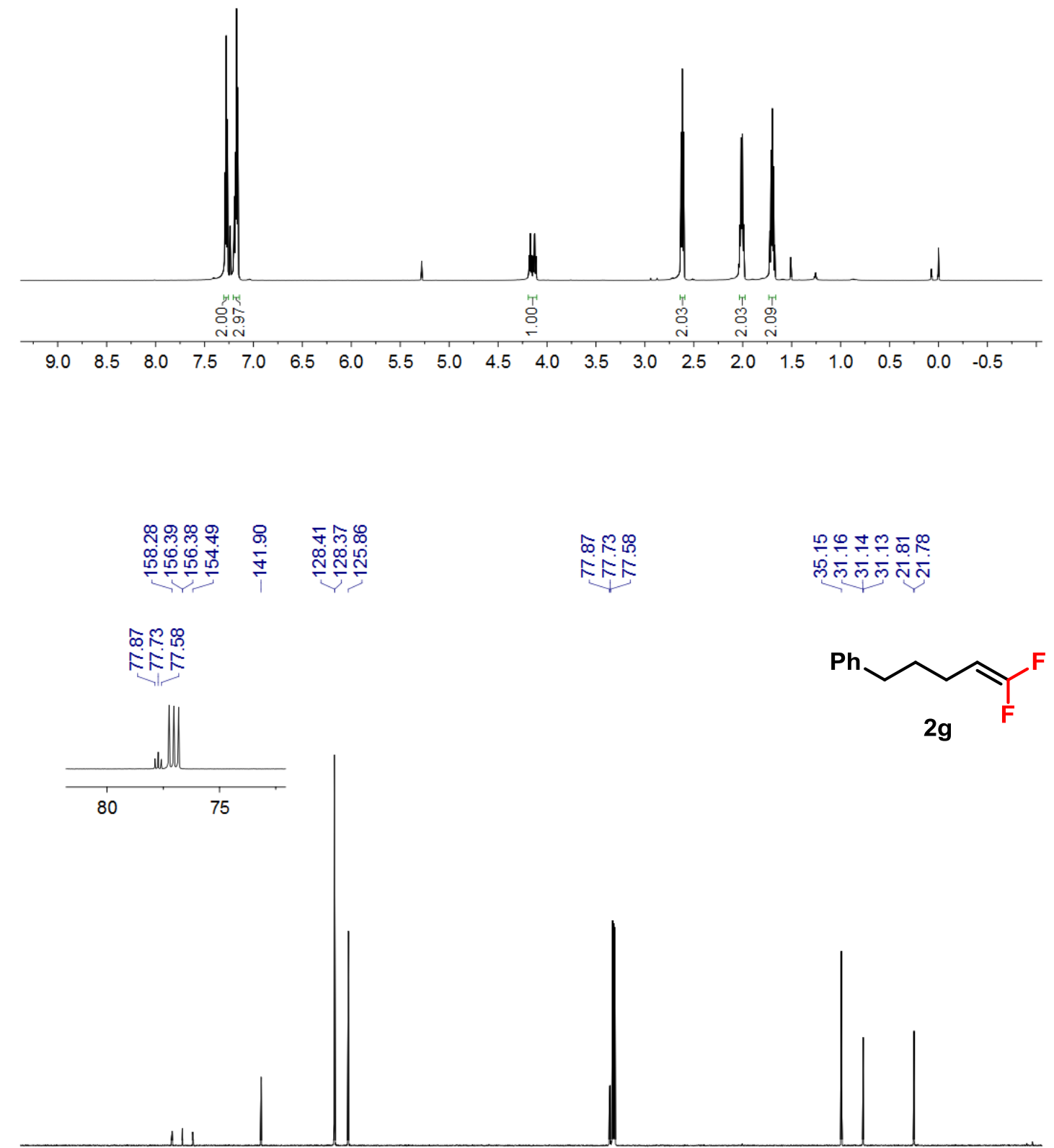

$\begin{array}{lllllllllllllllllll}180 & 170 & 160 & 150 & 140 & 130 & 120 & 110 & 100 & 90 & 80 & 70 & 60 & 50 & 40 & 30 & 20 & 10 & 0\end{array}$ 


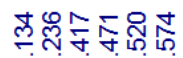

कंषिके

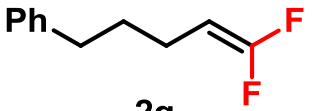

$2 g$

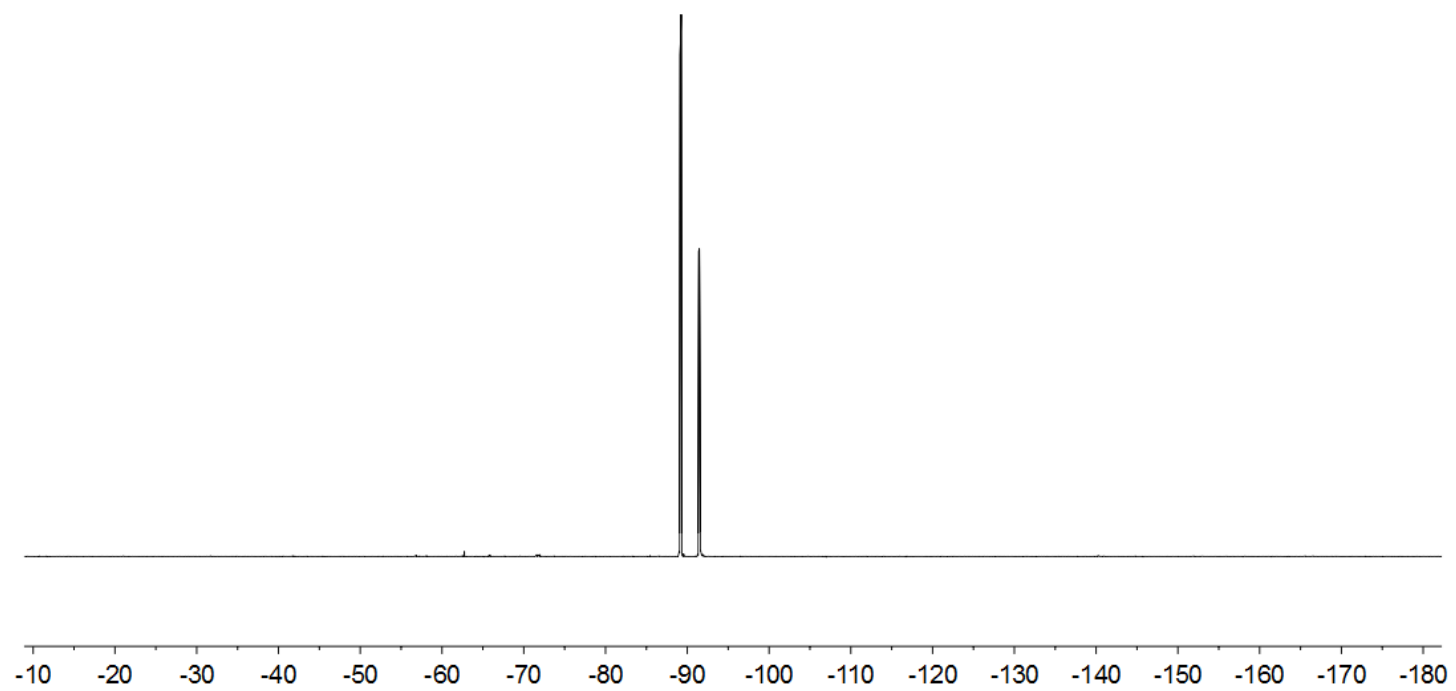

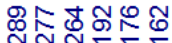

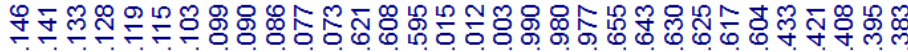

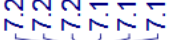

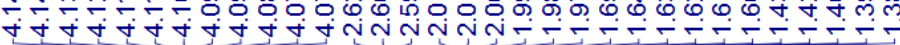

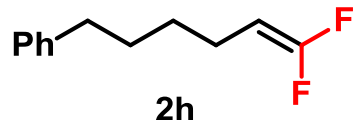

$2 h$

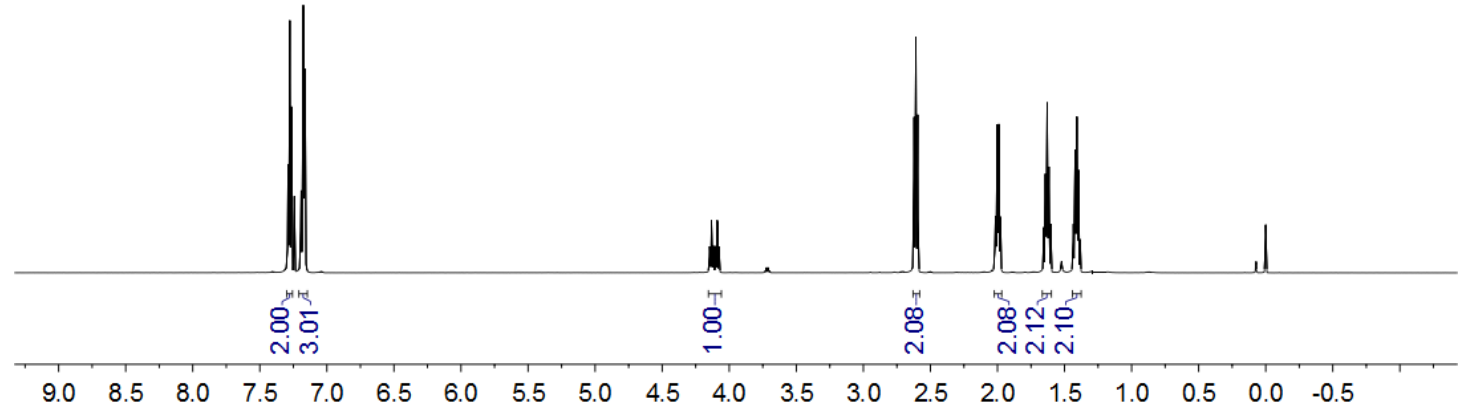



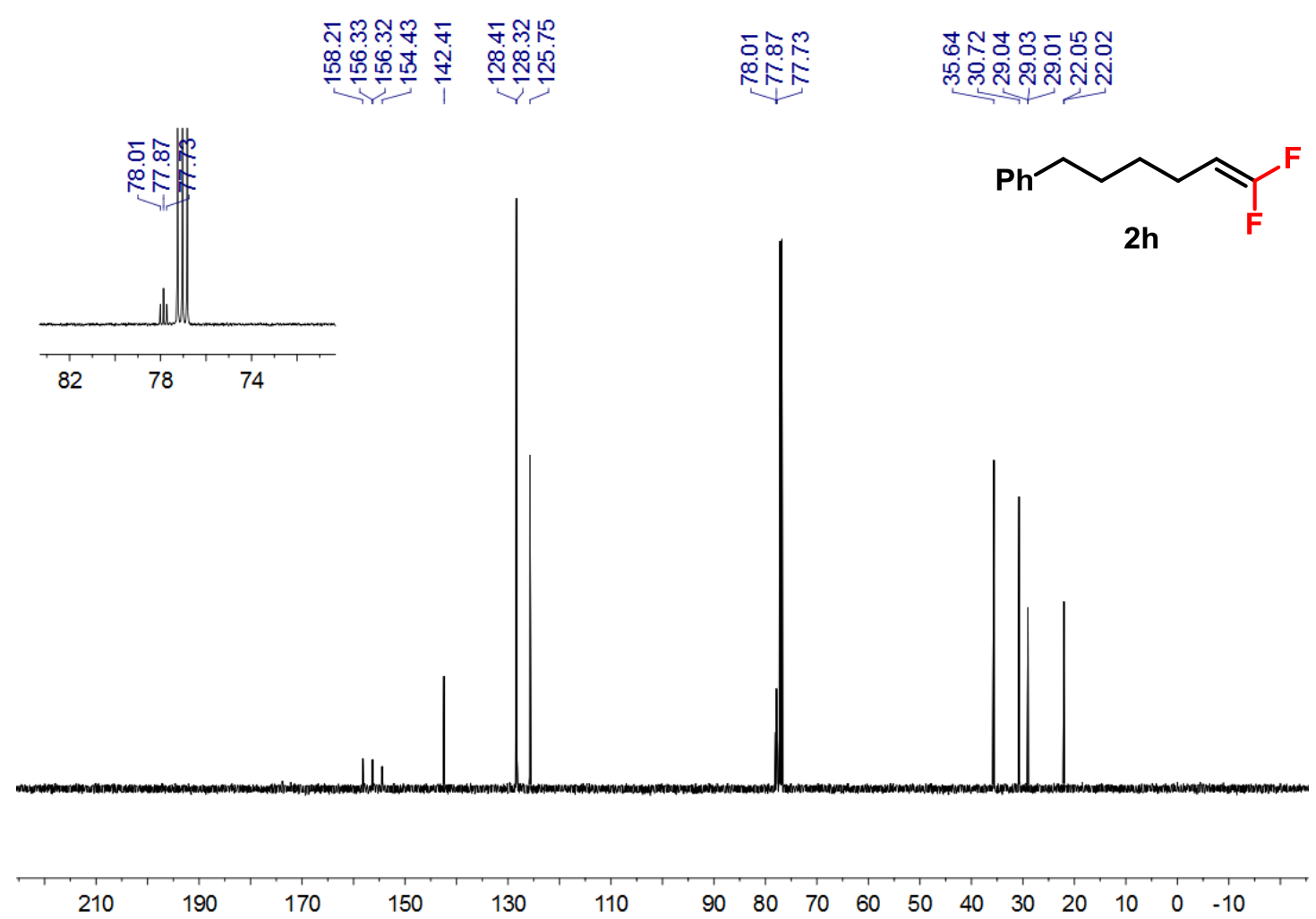

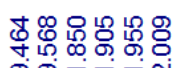

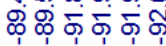

ᄂ

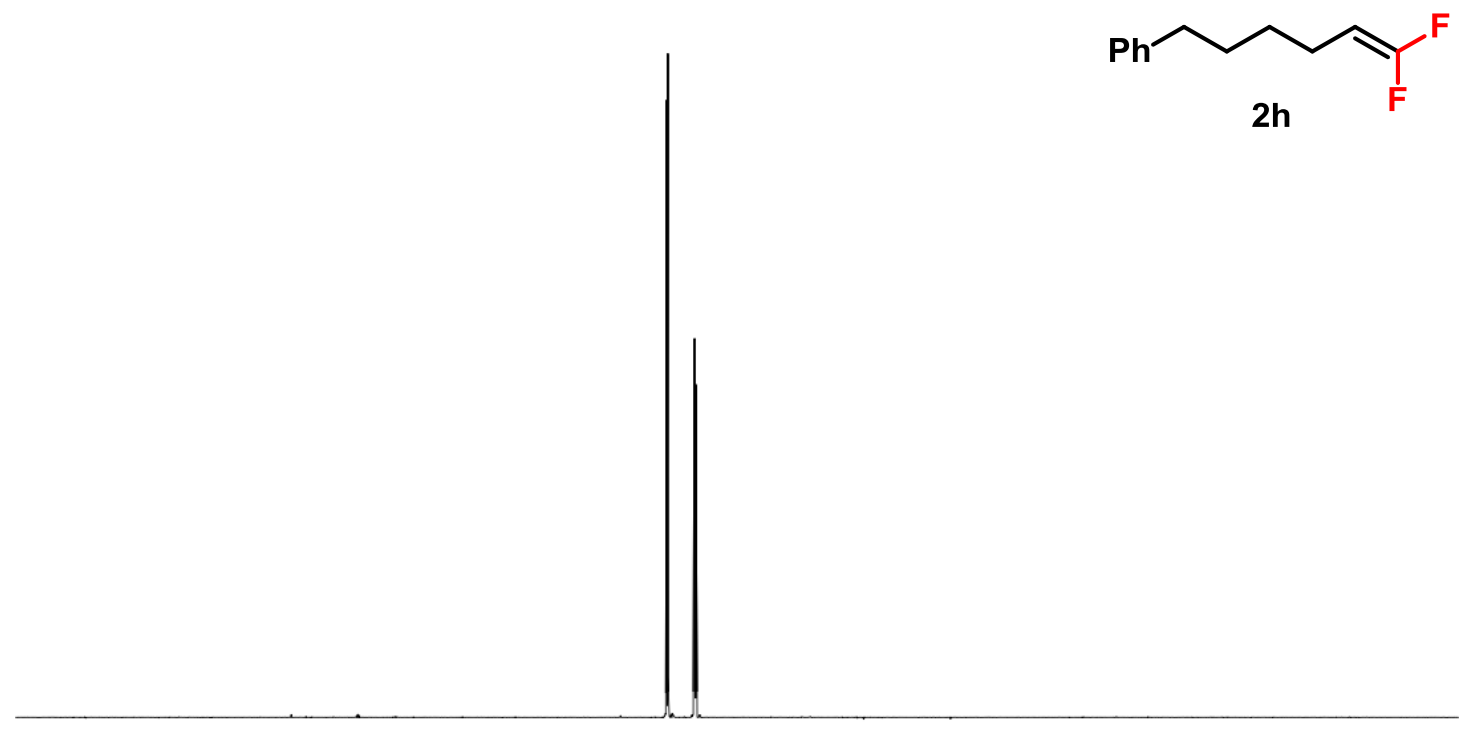

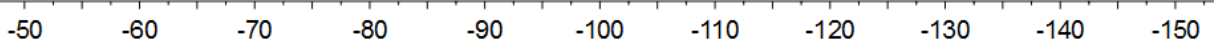



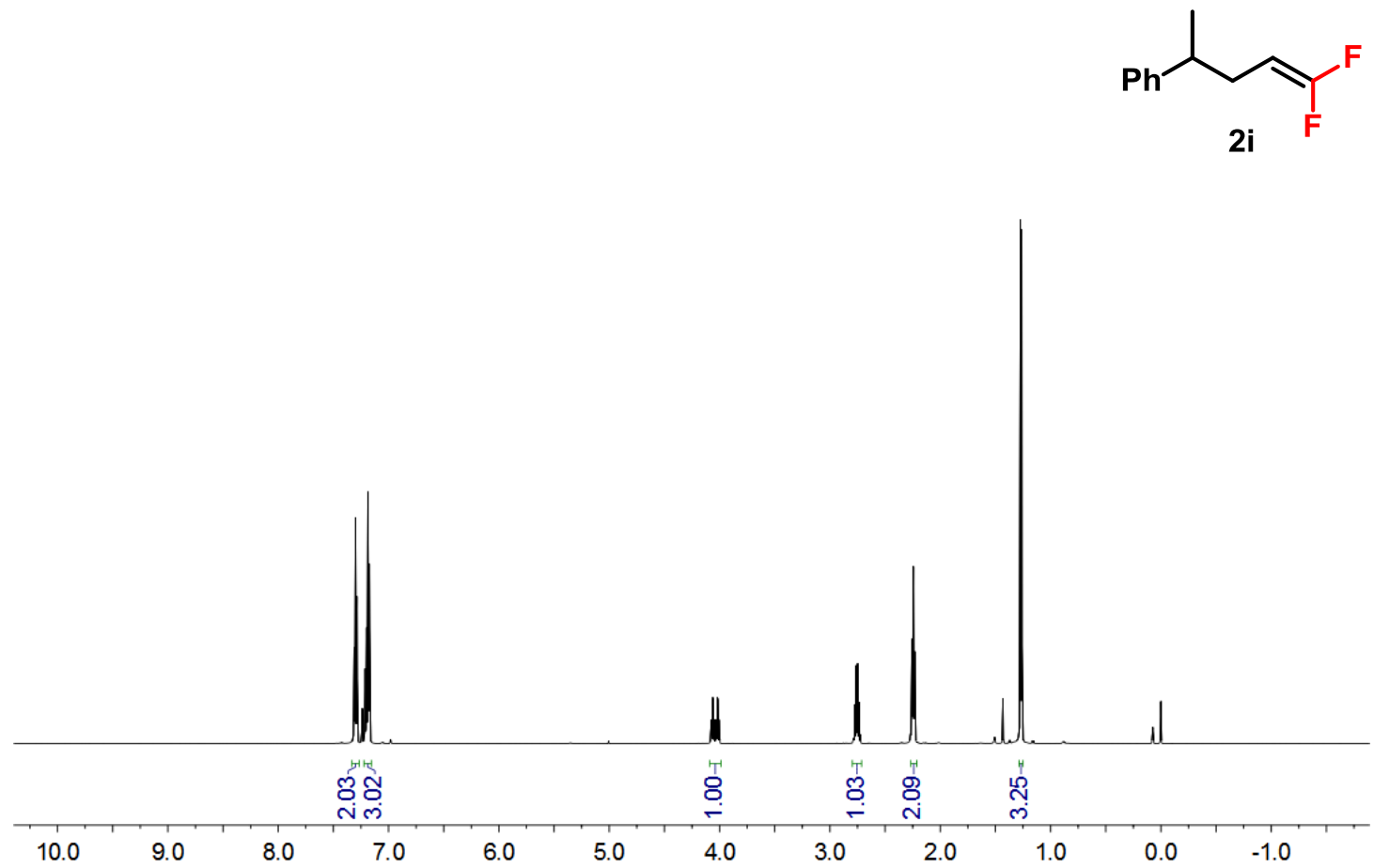

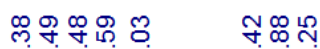

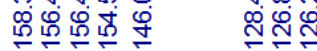

进轨品

유뉴

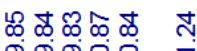

लिम्लिएल्ले స

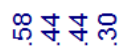

ए人iर
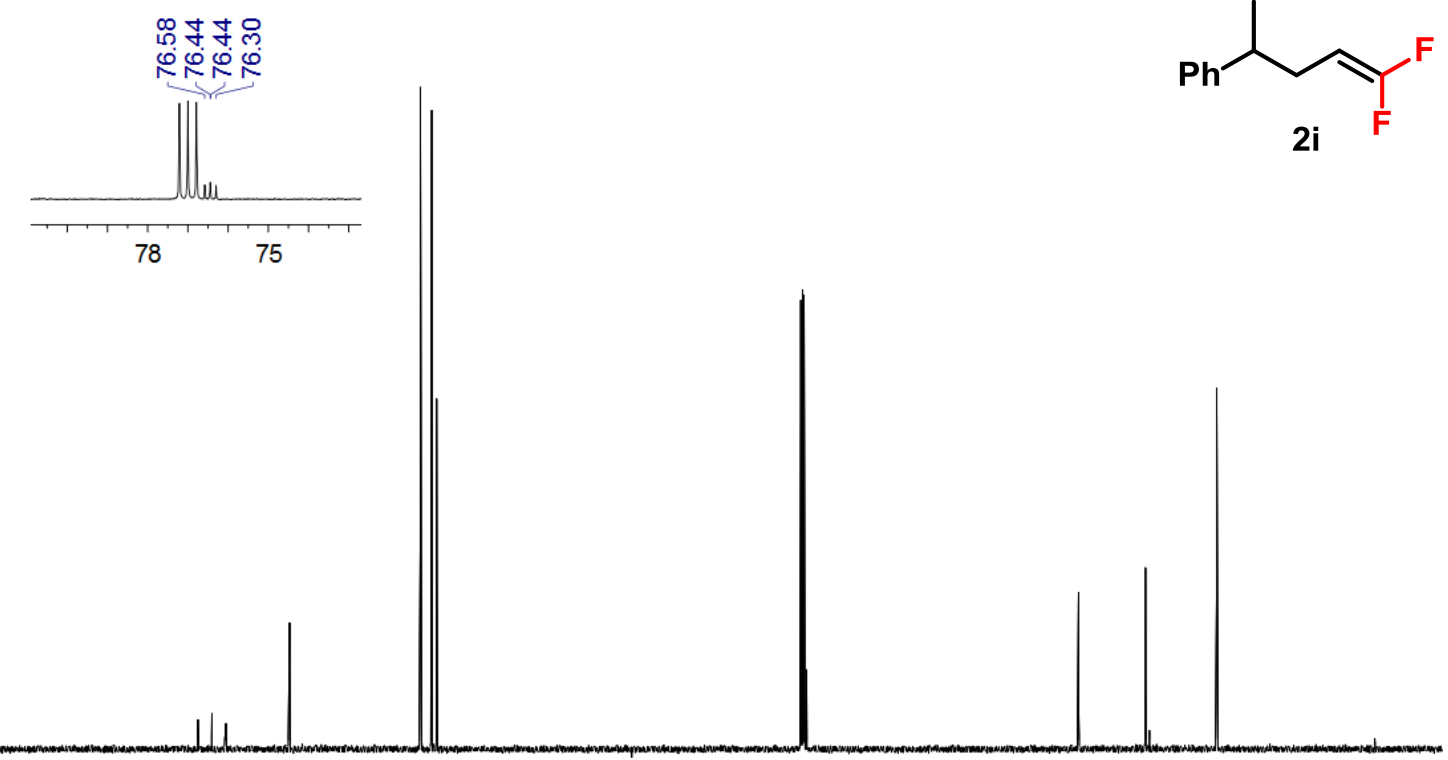

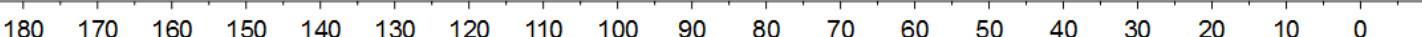




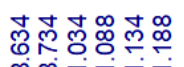

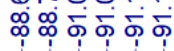

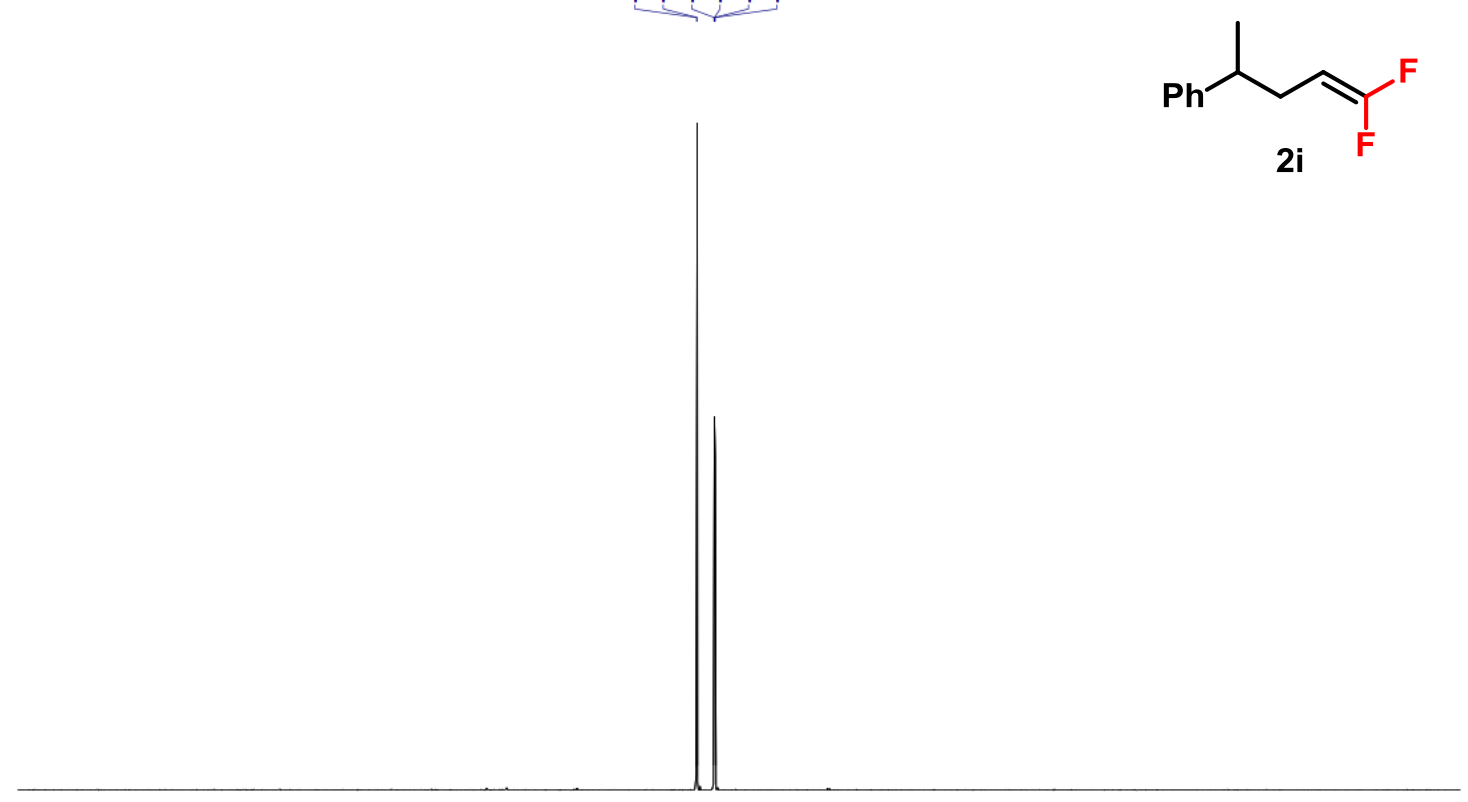

\begin{tabular}{llllllllllllllll}
\hline 0 & -10 & -20 & -30 & -40 & -50 & -60 & -70 & -80 & -90 & -100 & -120 & -140 & -160 & -180
\end{tabular}

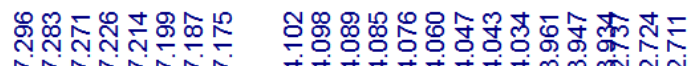

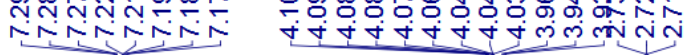
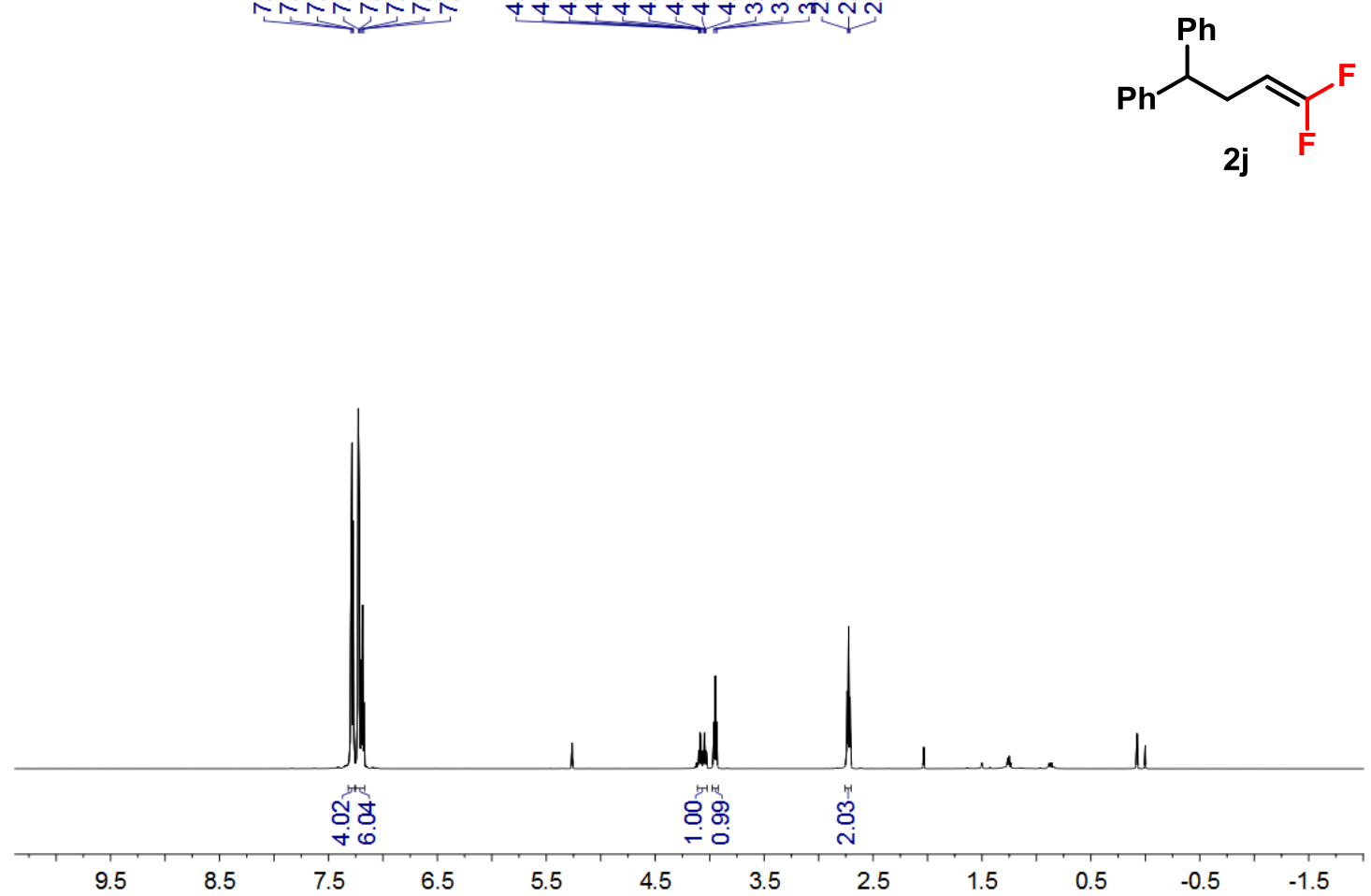


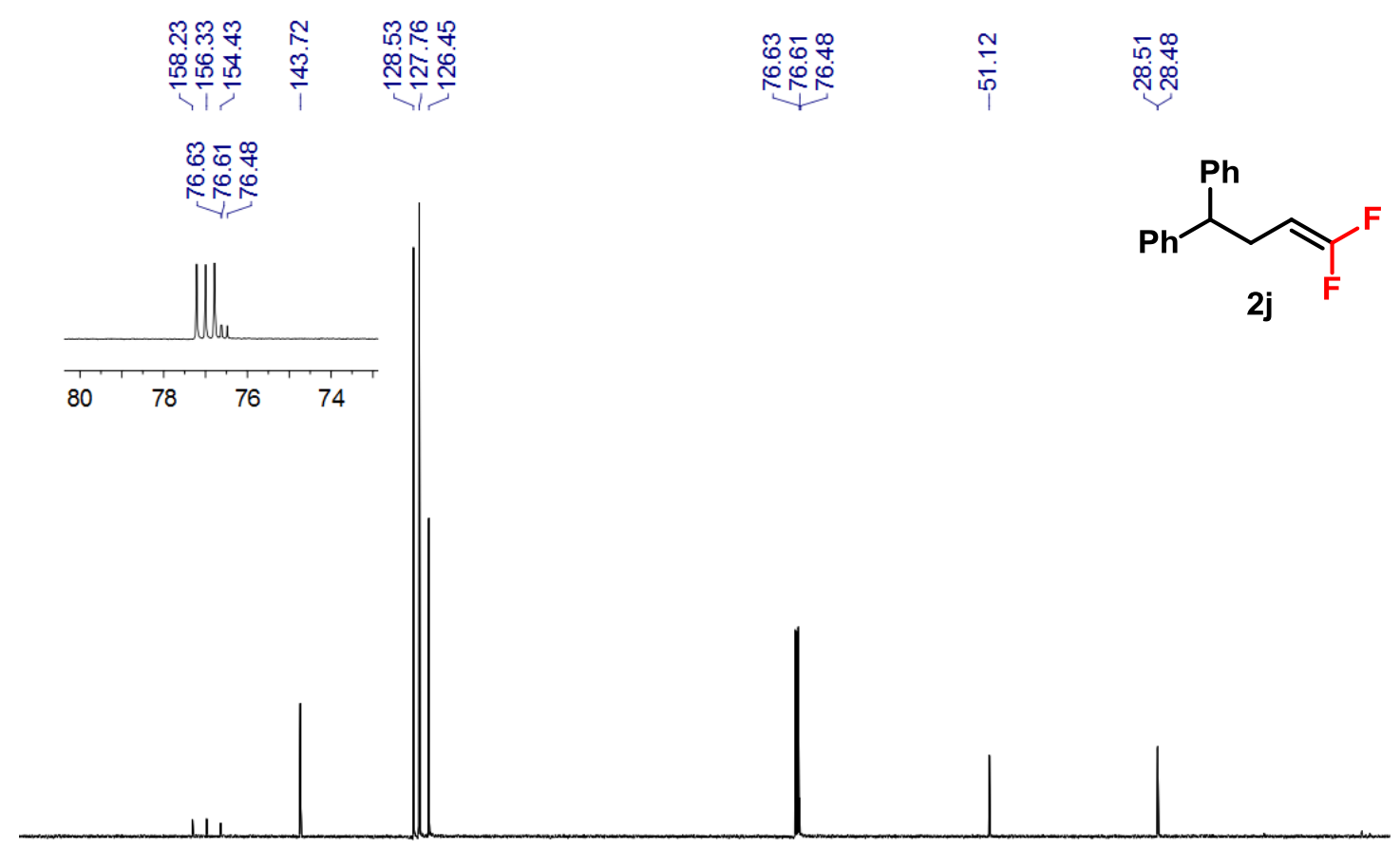

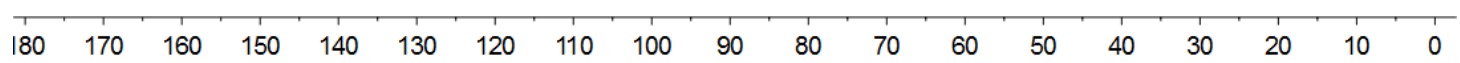

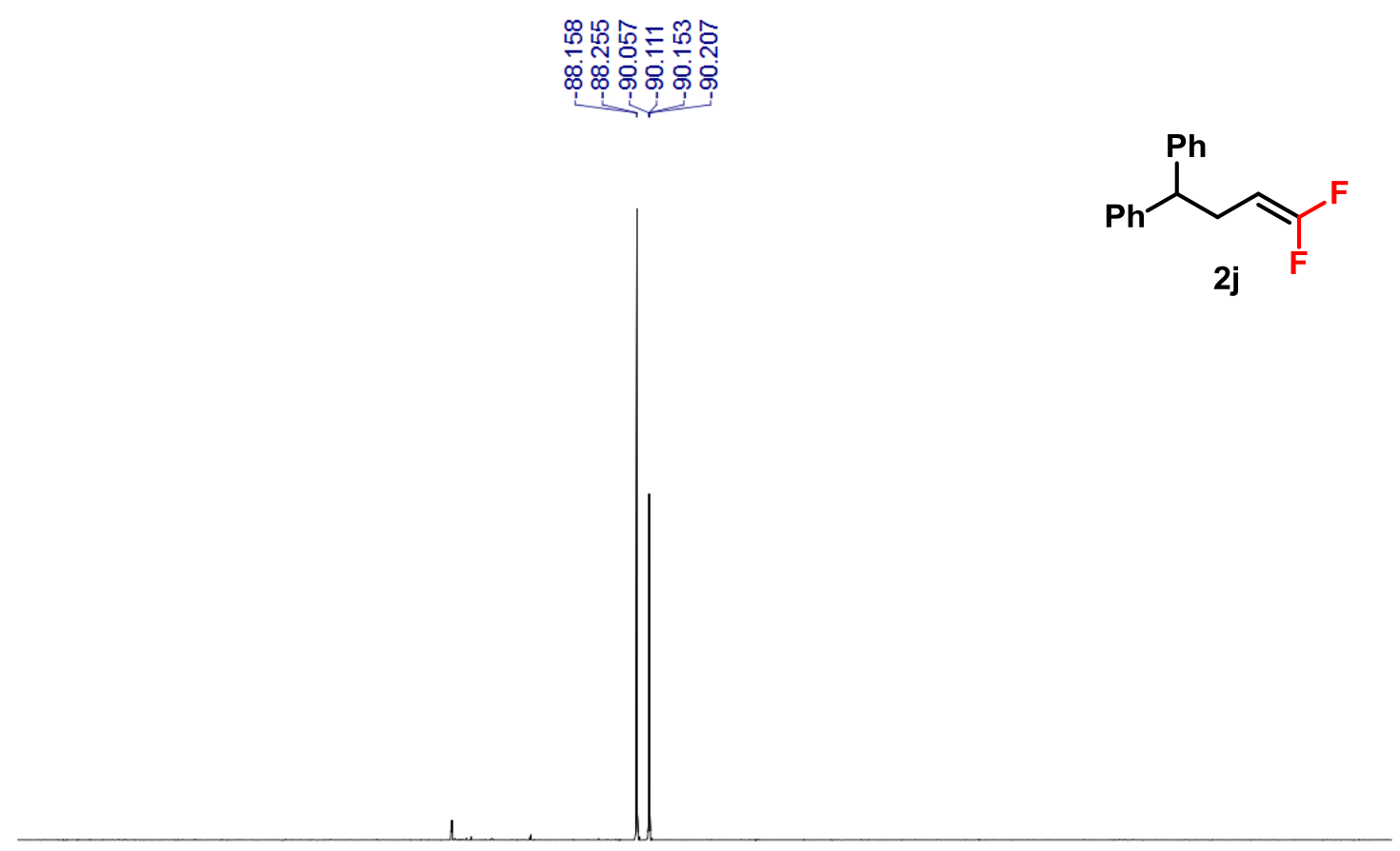

\begin{tabular}{|lllllllllllllll}
0 & -10 & -20 & -30 & -40 & -50 & -60 & -70 & -80 & -90 & -110 & -130 & -150 & -170 & -190
\end{tabular} 


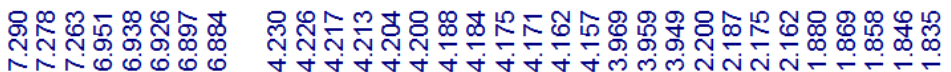

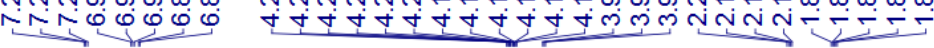
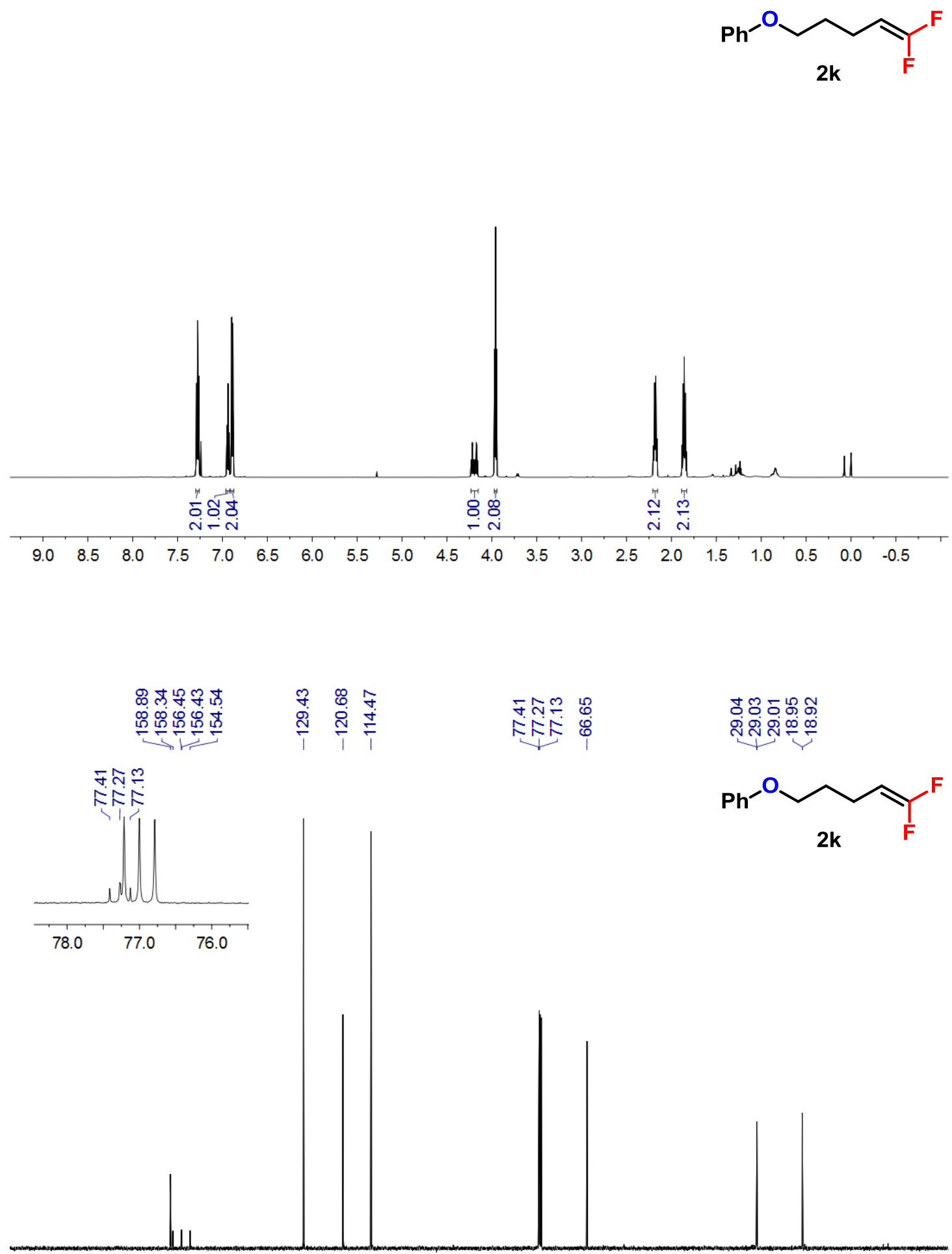

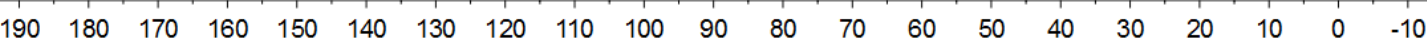




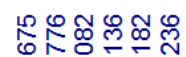

$\infty$

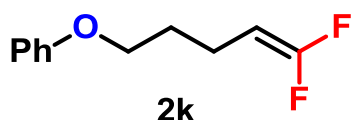

2k

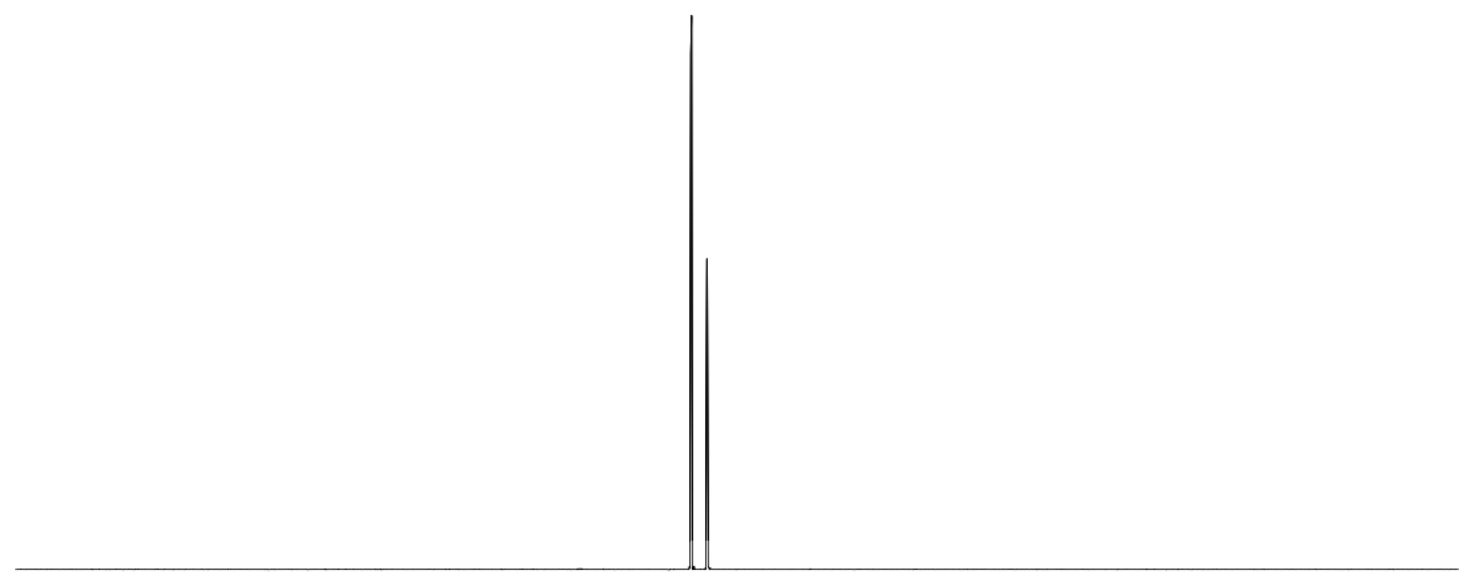

\begin{tabular}{llllllllllllllll}
\hline 10 & 0 & -10 & -20 & -30 & -40 & -50 & -60 & -70 & -80 & -90 & -110 & -130 & -150 & -170 & -190
\end{tabular}

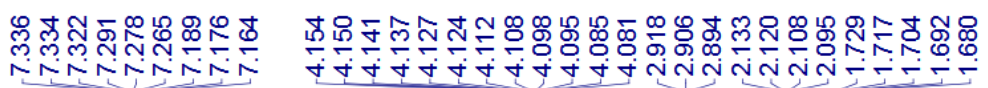
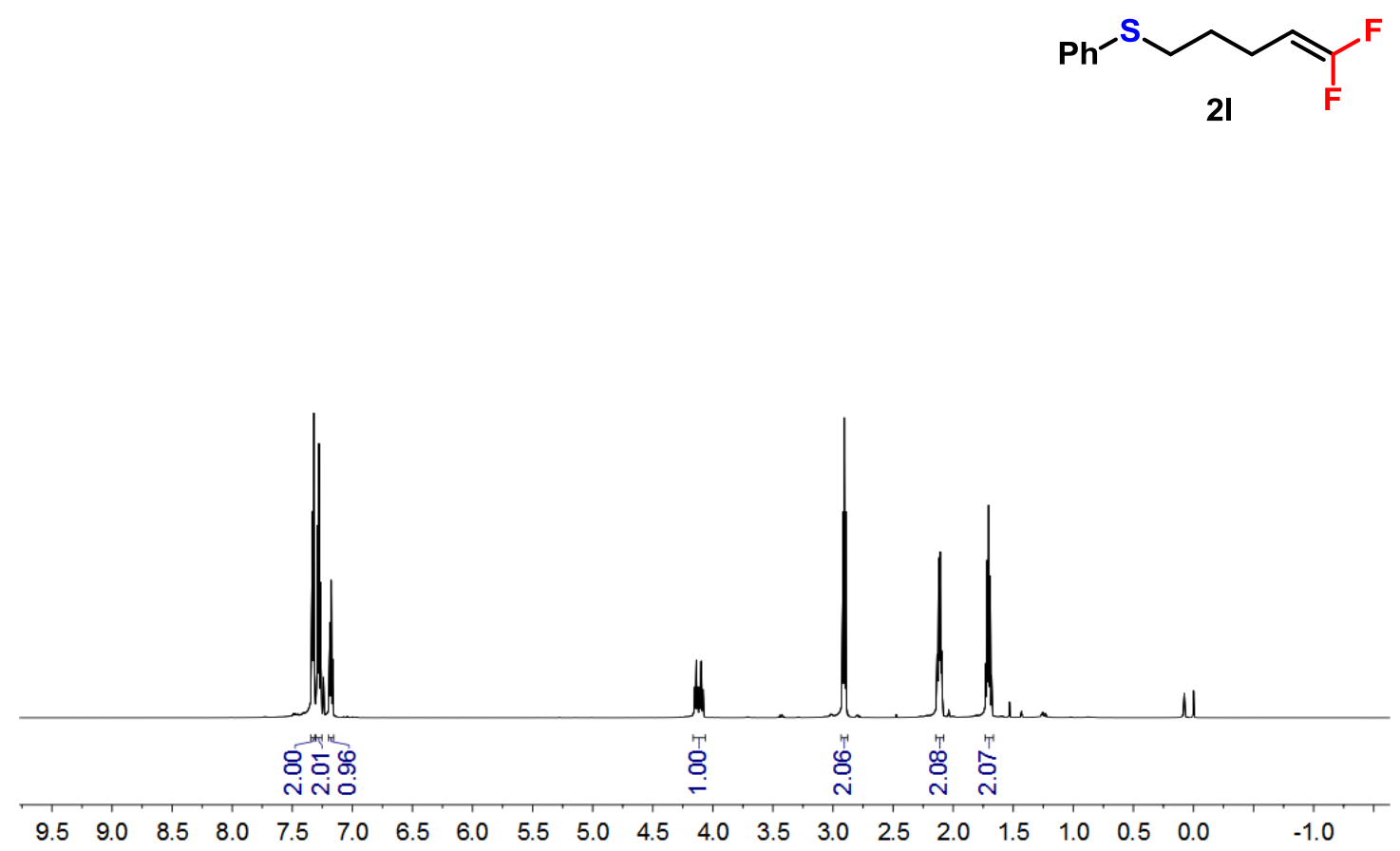


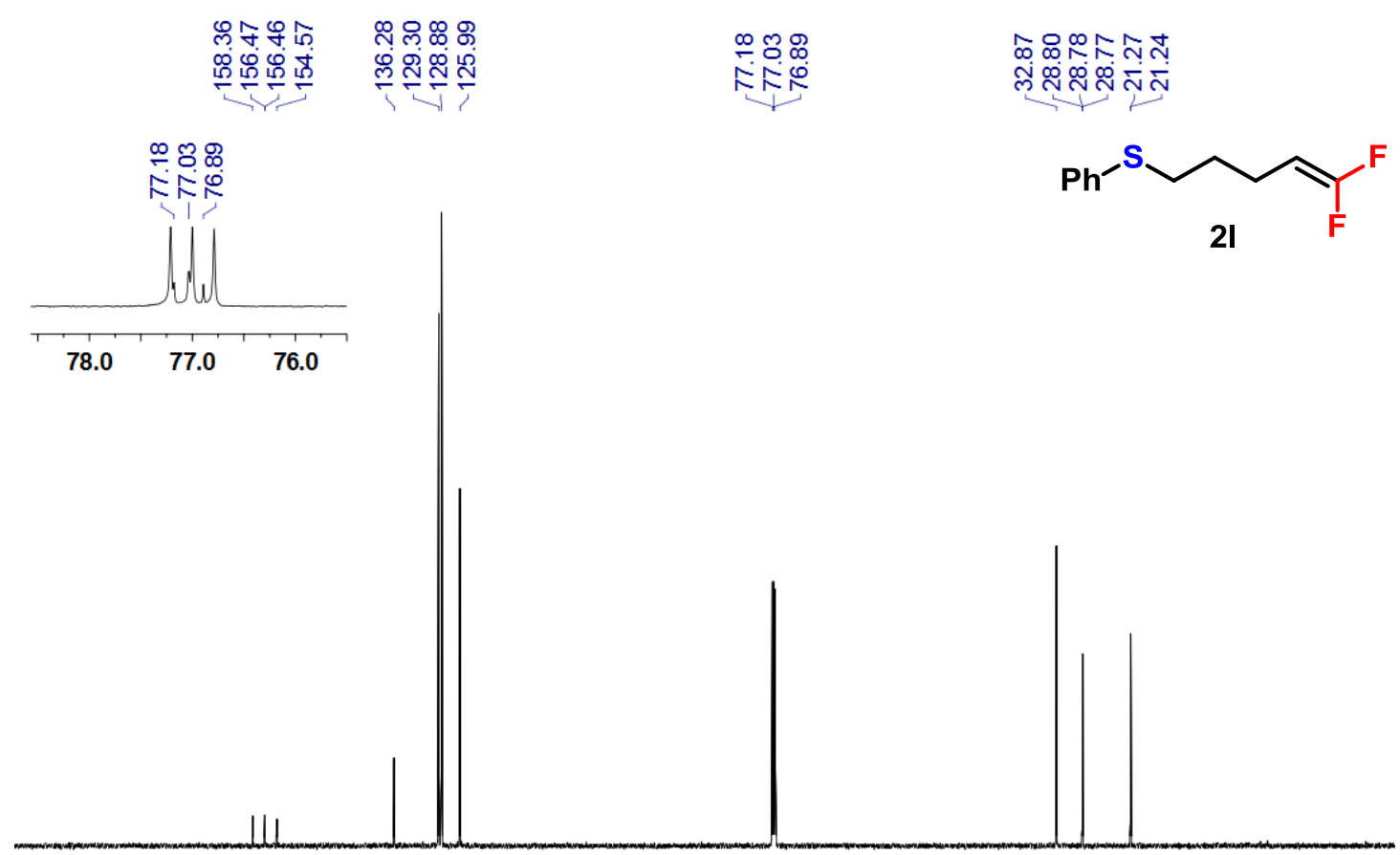

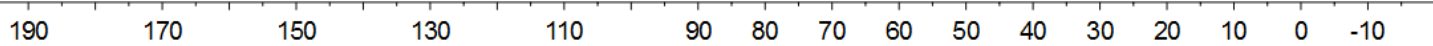

บำ

œ ळ ᄋ 요
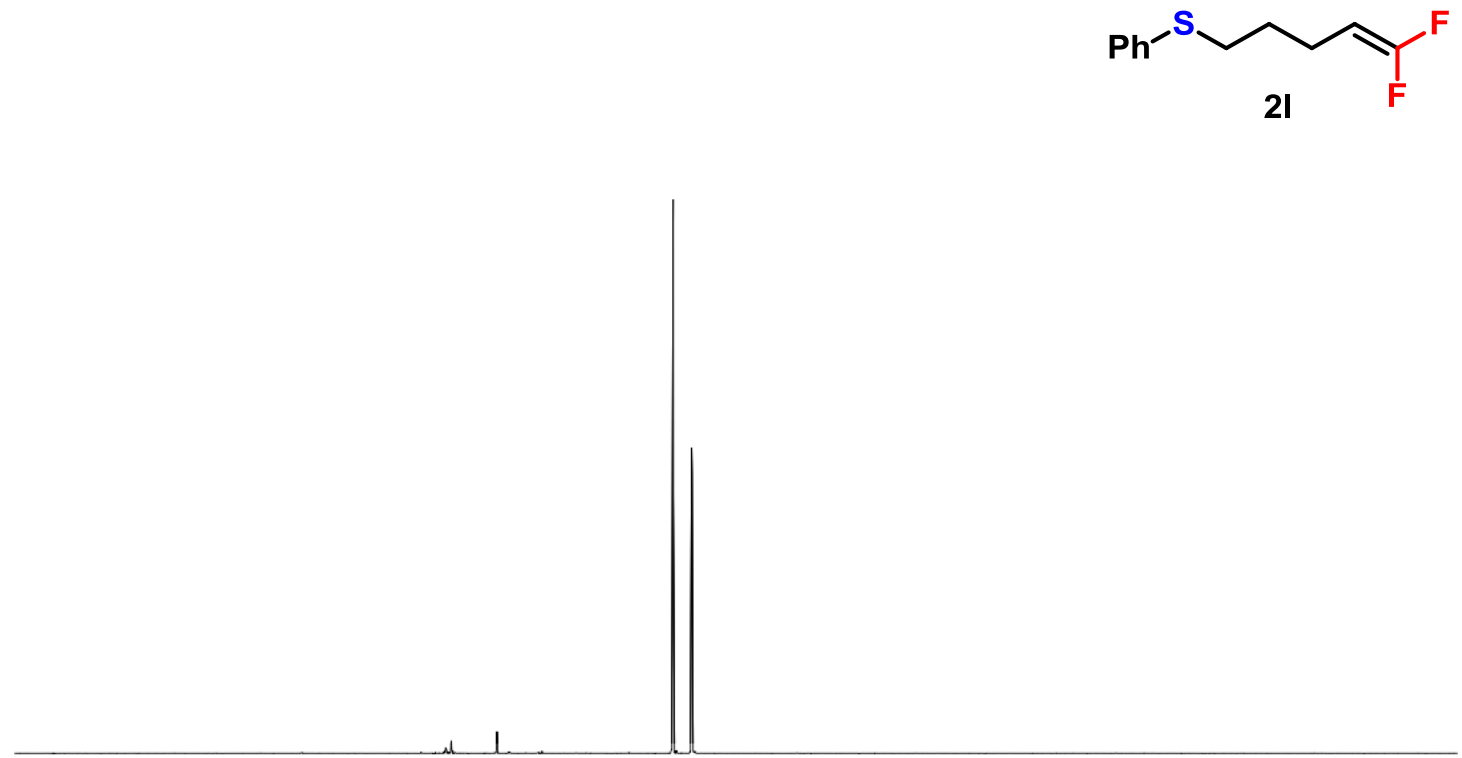

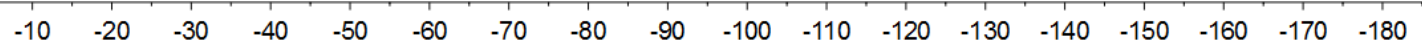




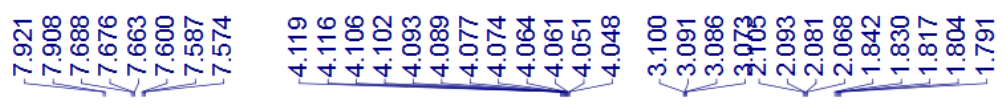
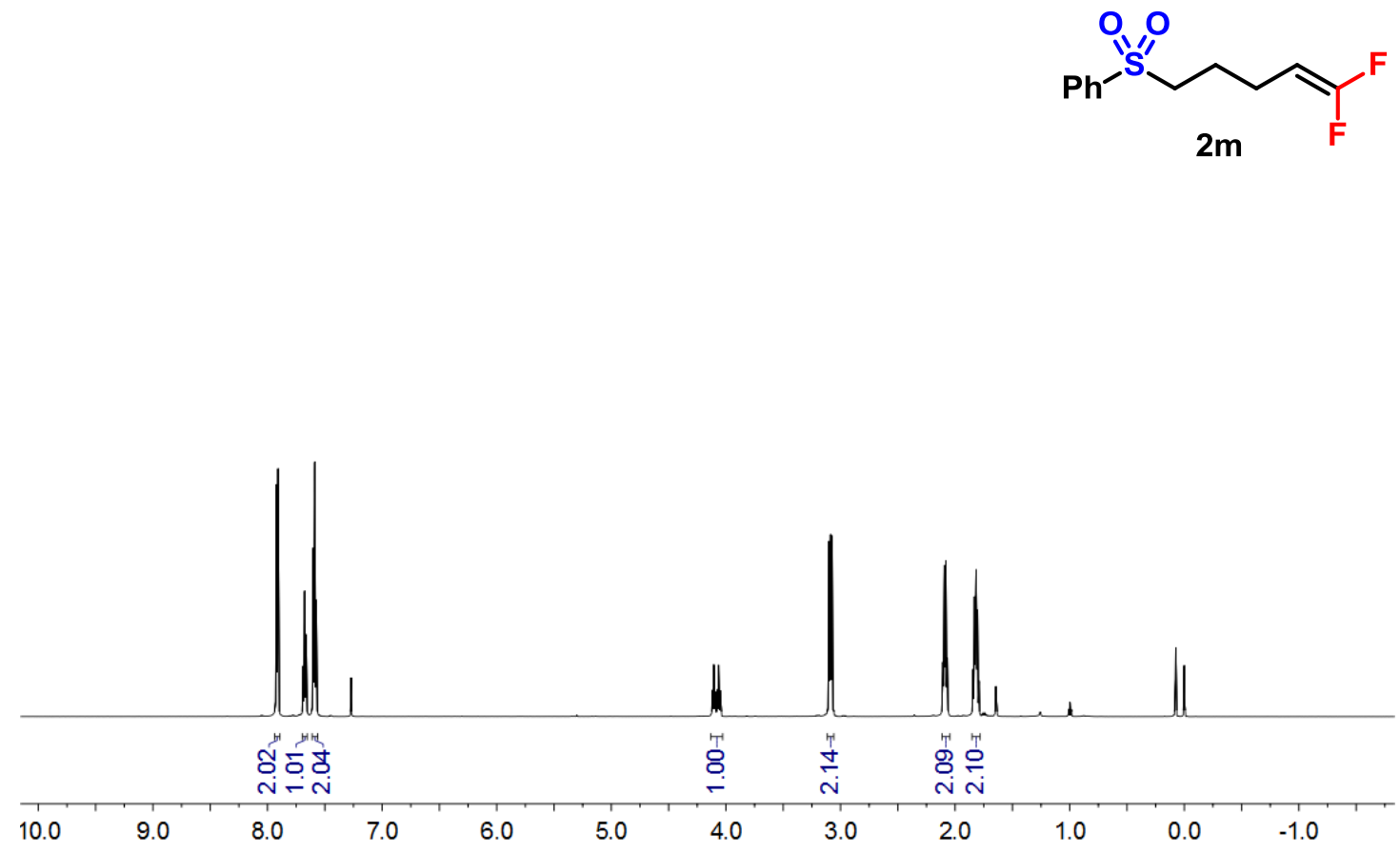

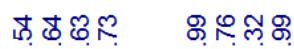

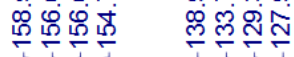

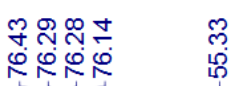

守年尔禺

สูกส่ักิ

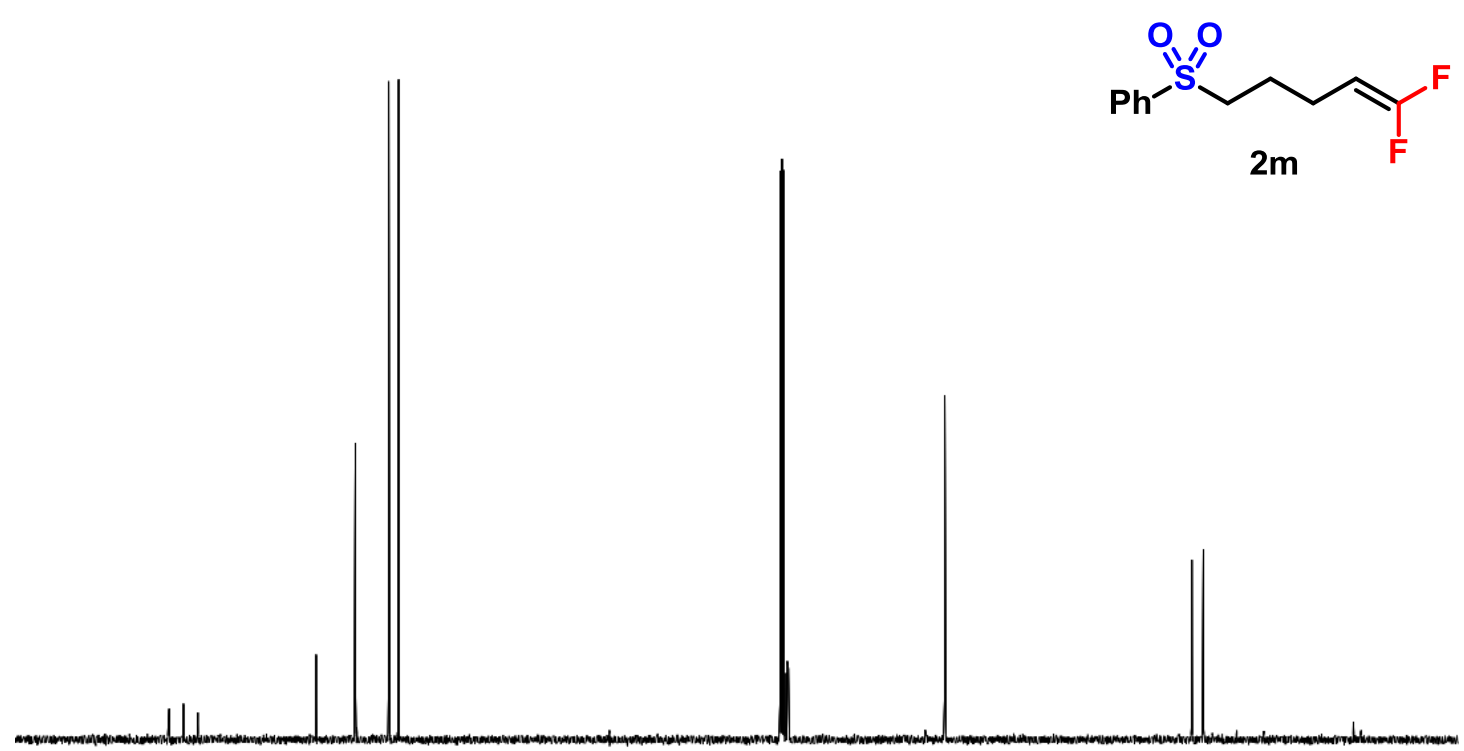

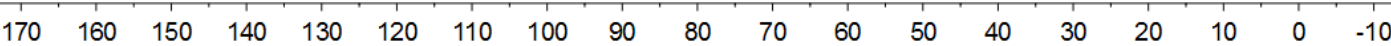




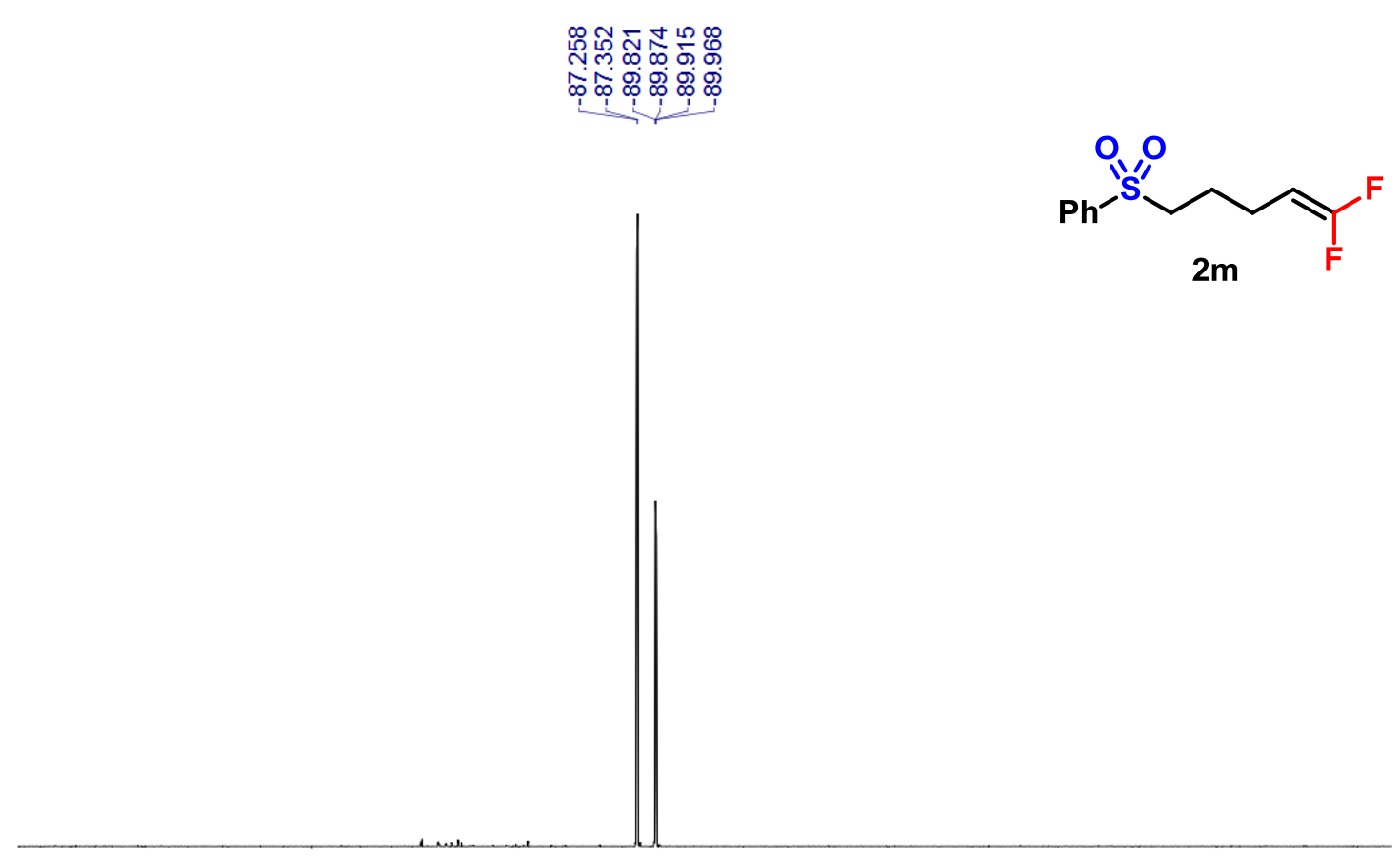

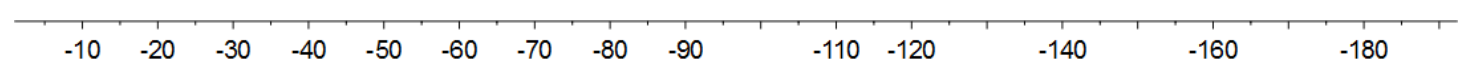

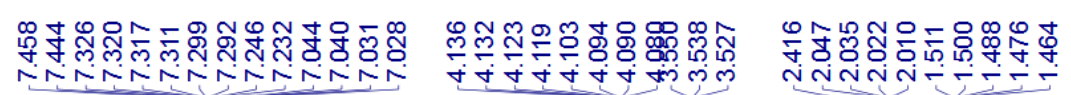

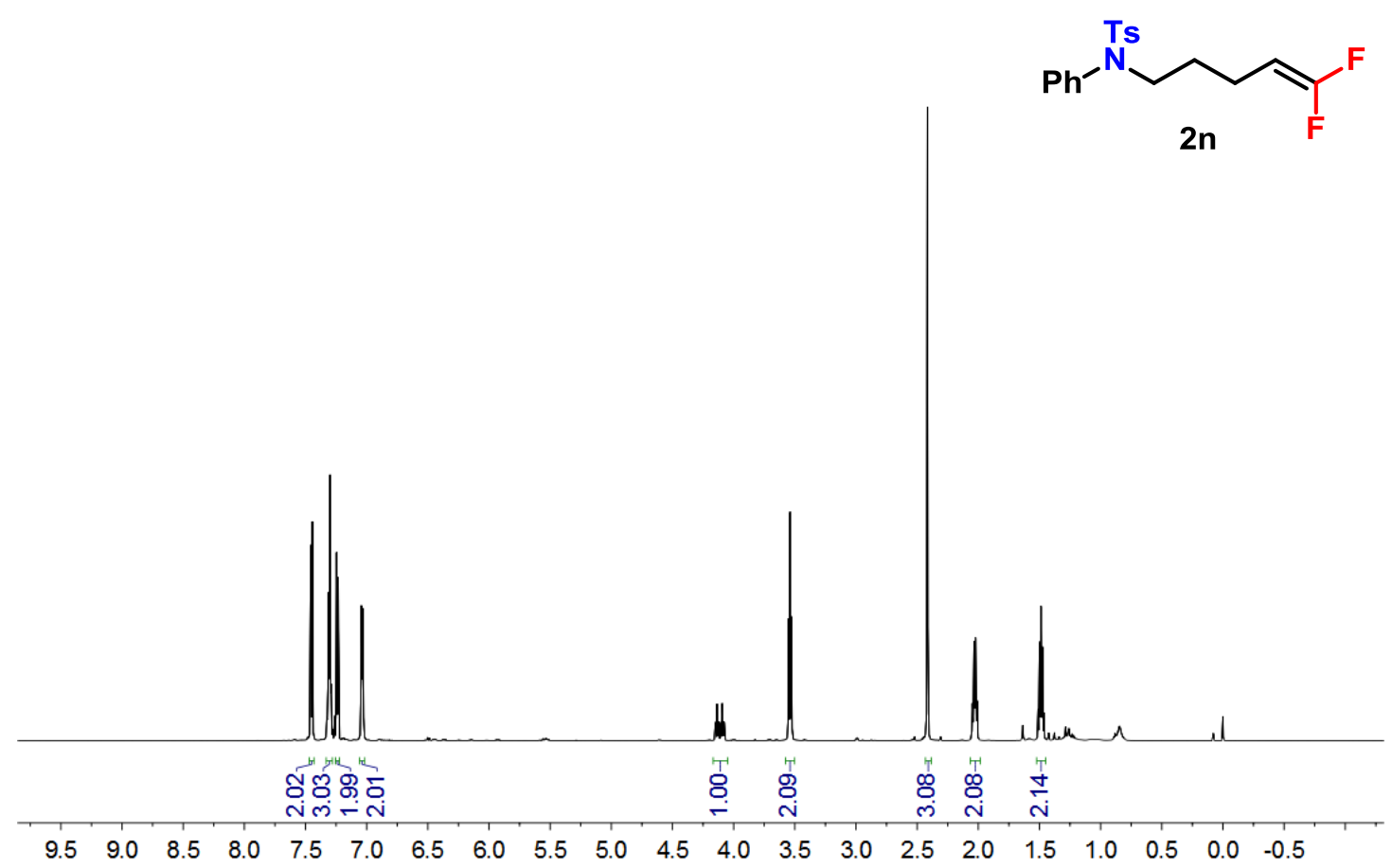




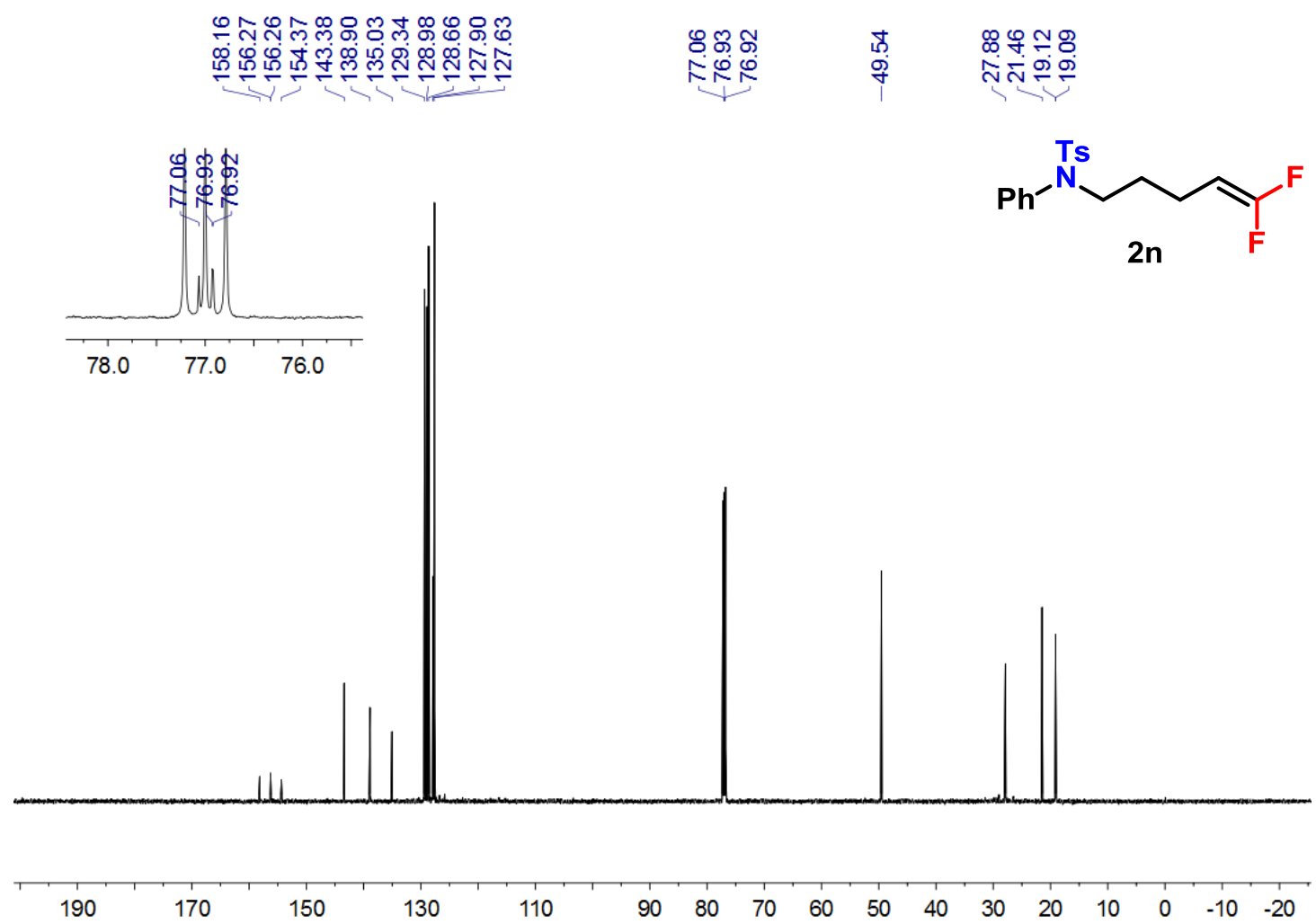

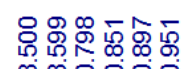

œ চ চ্চি
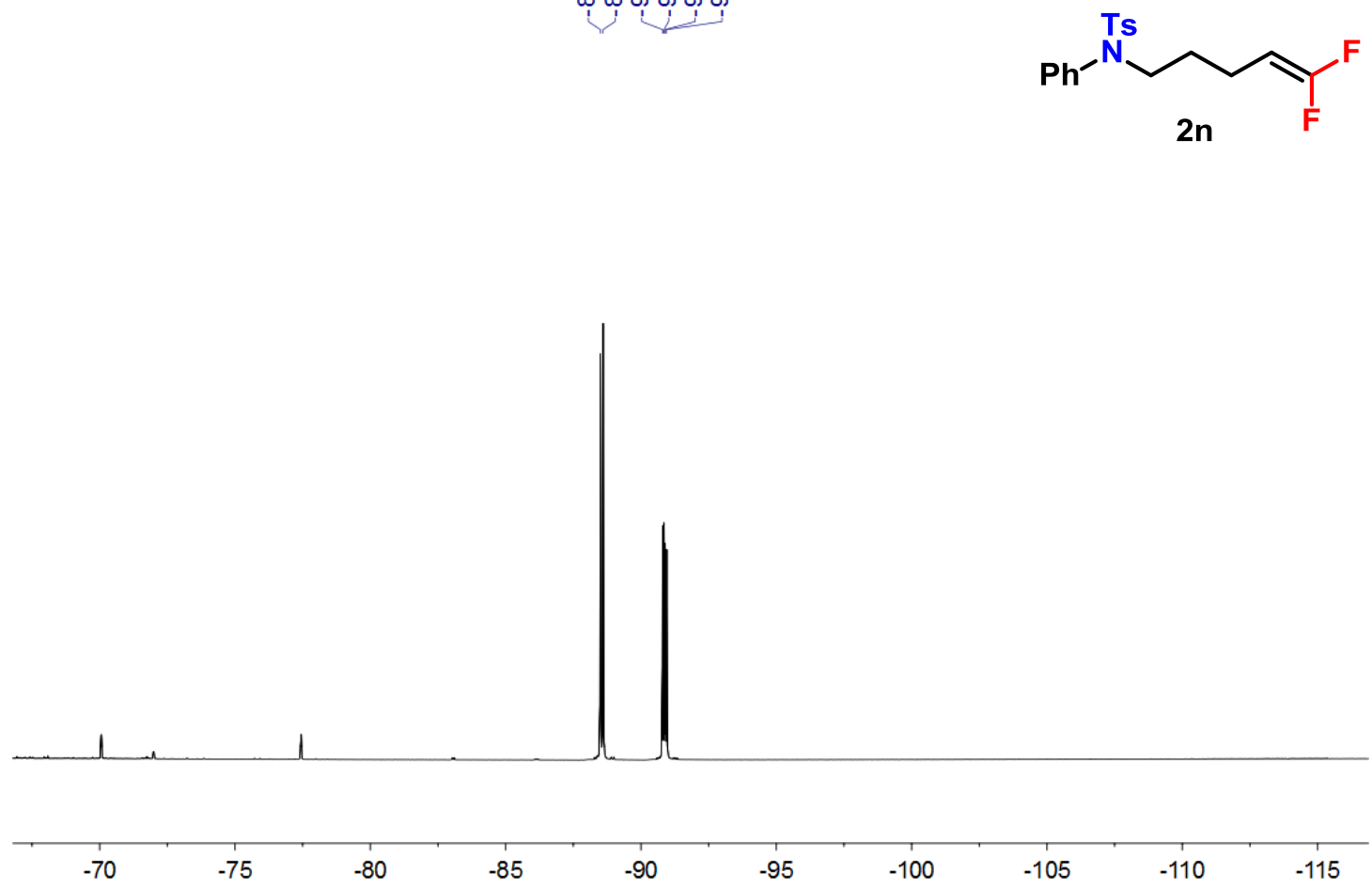


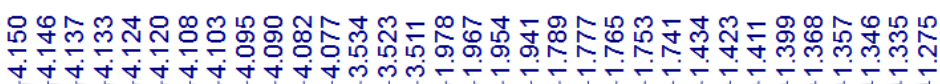
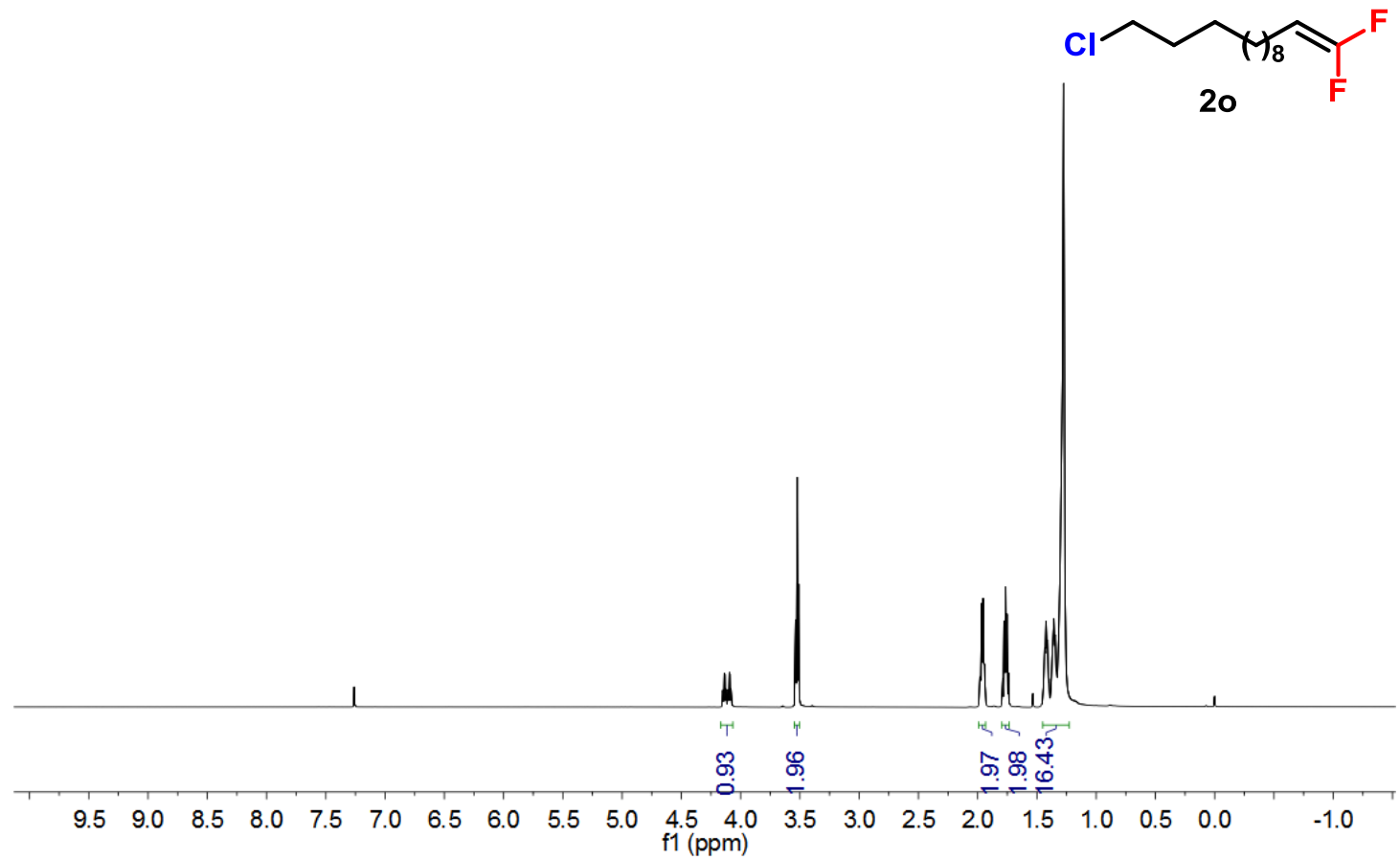

느뉴ำำ

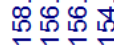

$\div 8 \%$

乐足

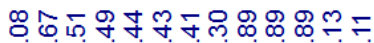

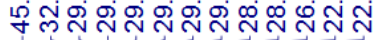

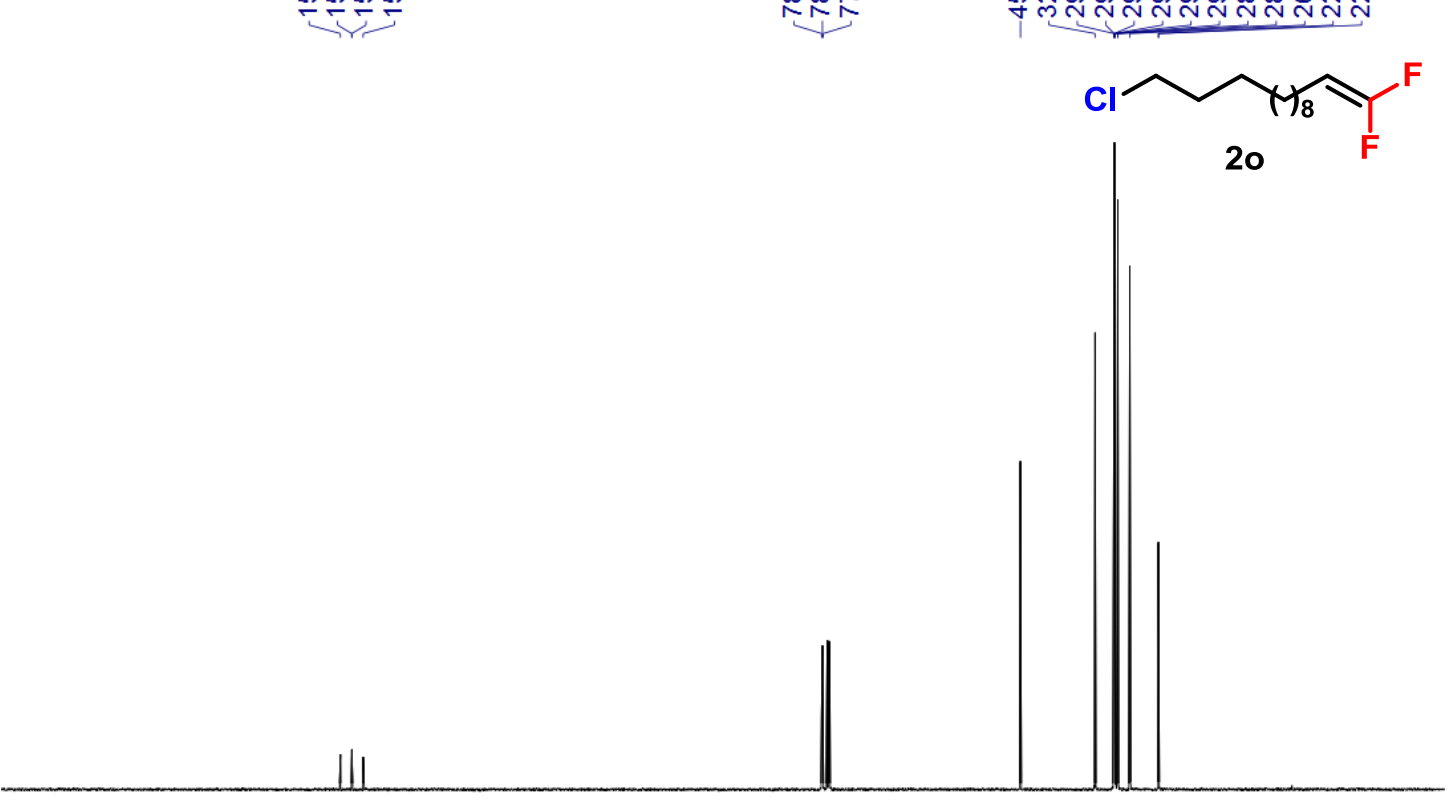

210

$190 \quad 170 \quad 150$

130

$110 \quad \begin{array}{r}90 \\ \mathrm{f} 1(\mathrm{ppm})\end{array}$

$\begin{array}{llllllllll}80 & 70 & 60 & 50 & 40 & 30 & 20 & 10 & 0 & -10\end{array}$ 


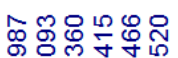

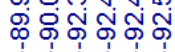
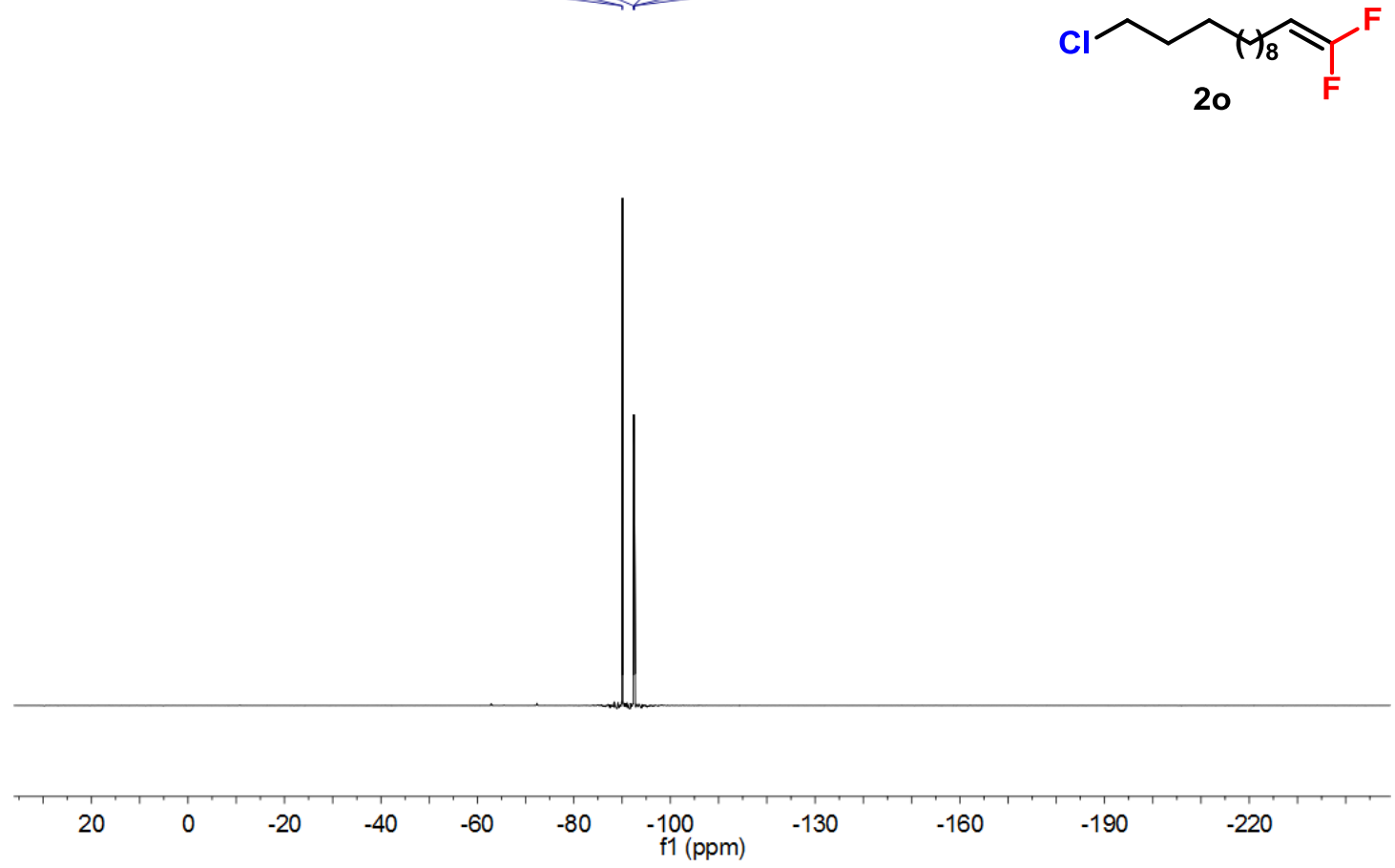

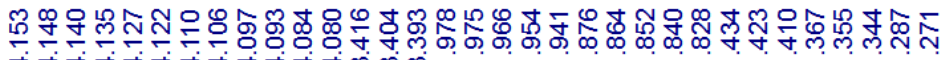

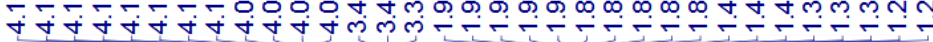

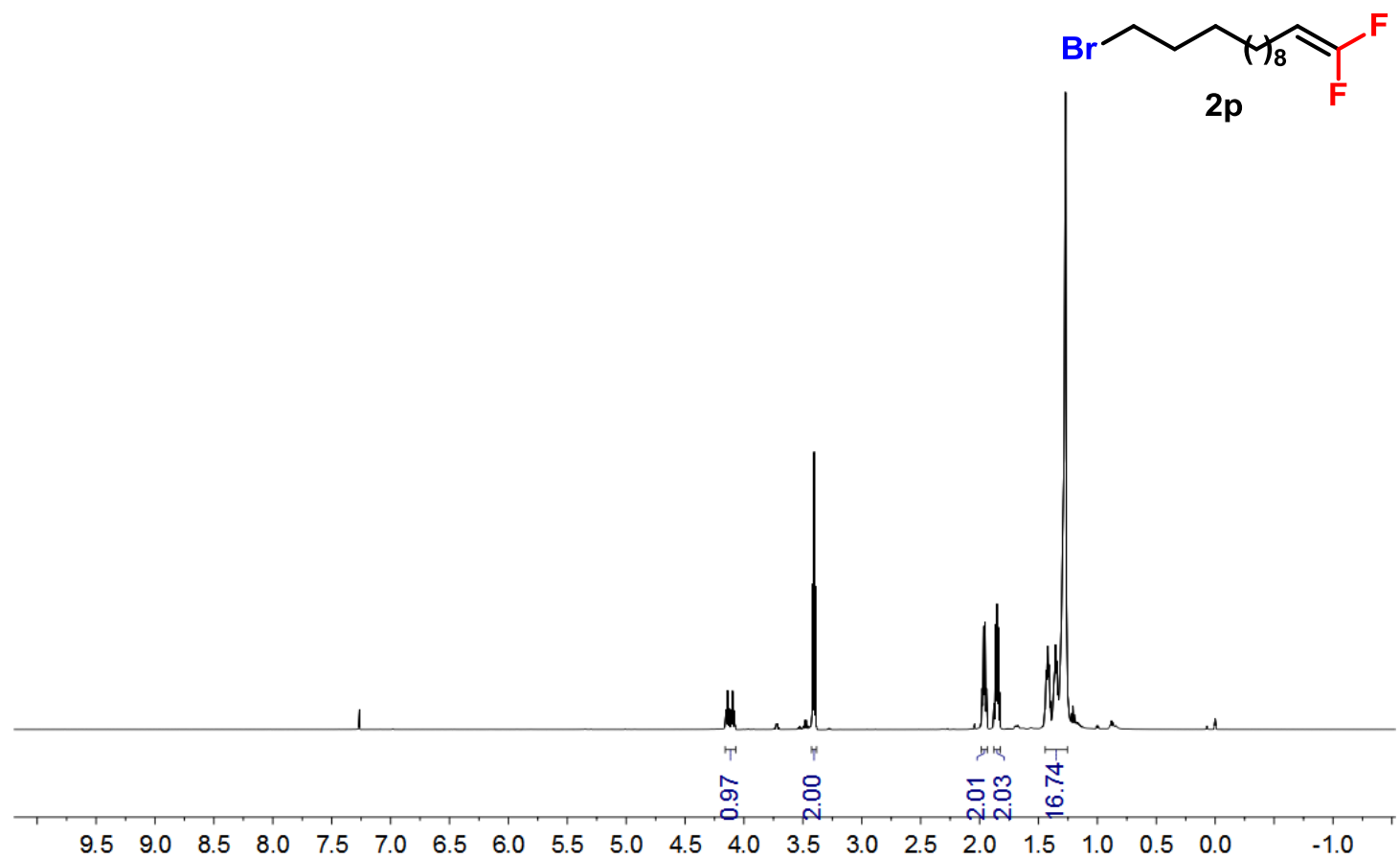




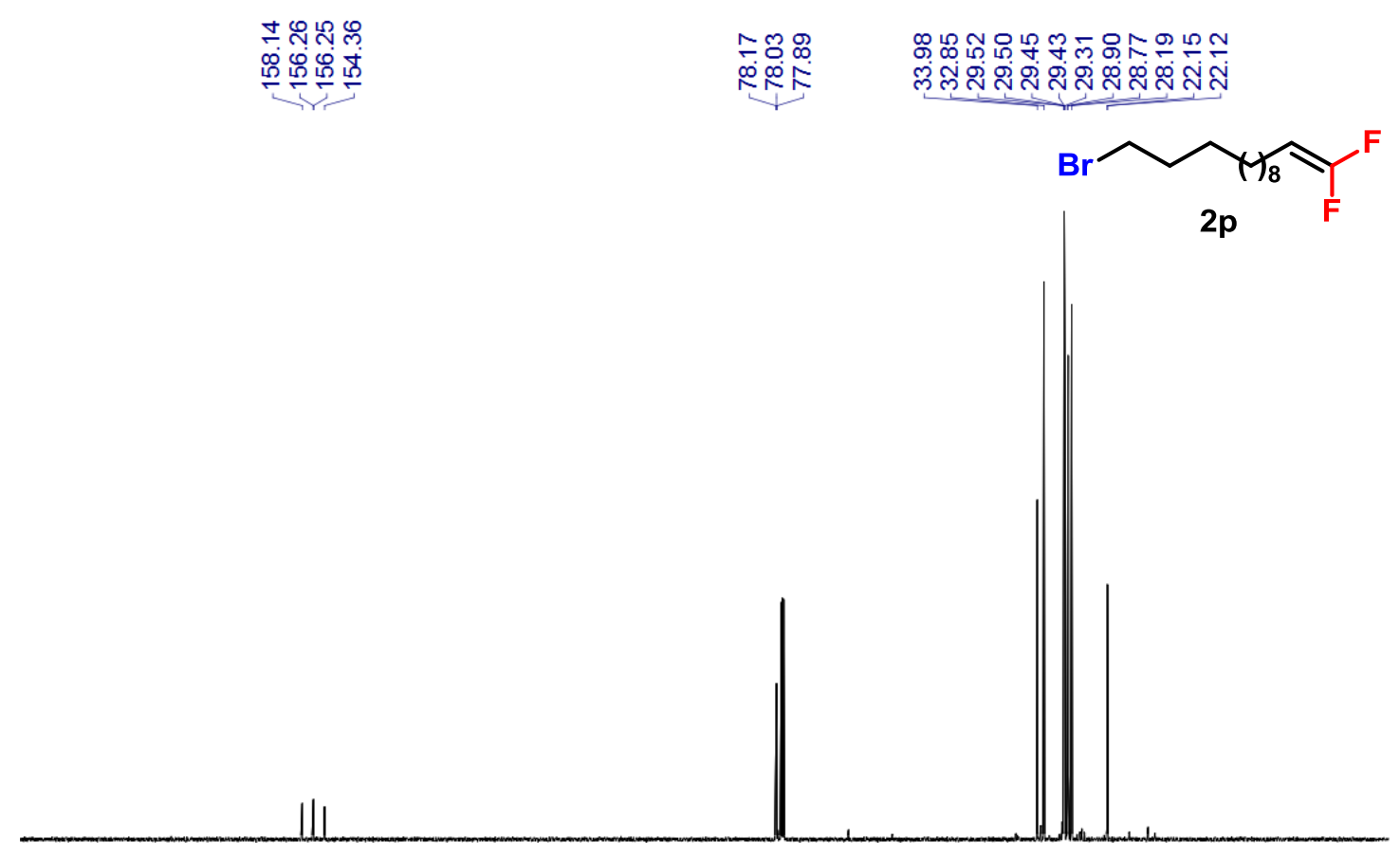

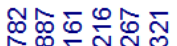

ळळ
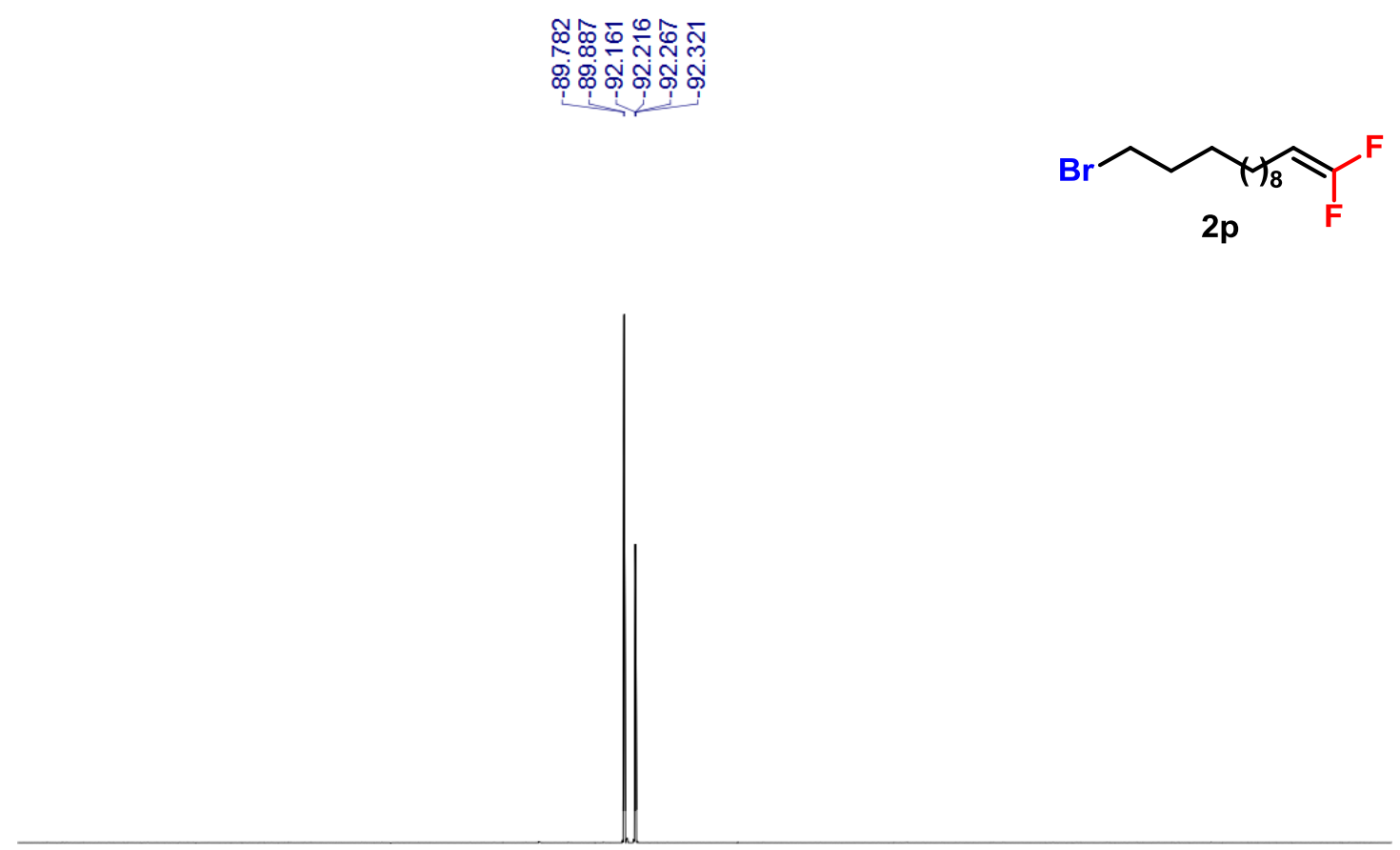

\begin{tabular}{llllllllllllllllllll}
\hline 190 & 170 & 150 & 130 & 110 & 90 & 80 & 70 & 60 & 50 & 40 & 30 & 20 & 10 & 0 & -10 & -20
\end{tabular}

\begin{tabular}{lllllllllll}
\hline & 0 & -20 & -40 & -60 & -80 & -100 & -130 & -160 & -190 & -220
\end{tabular}




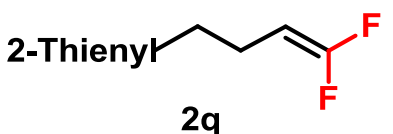

$2 q$

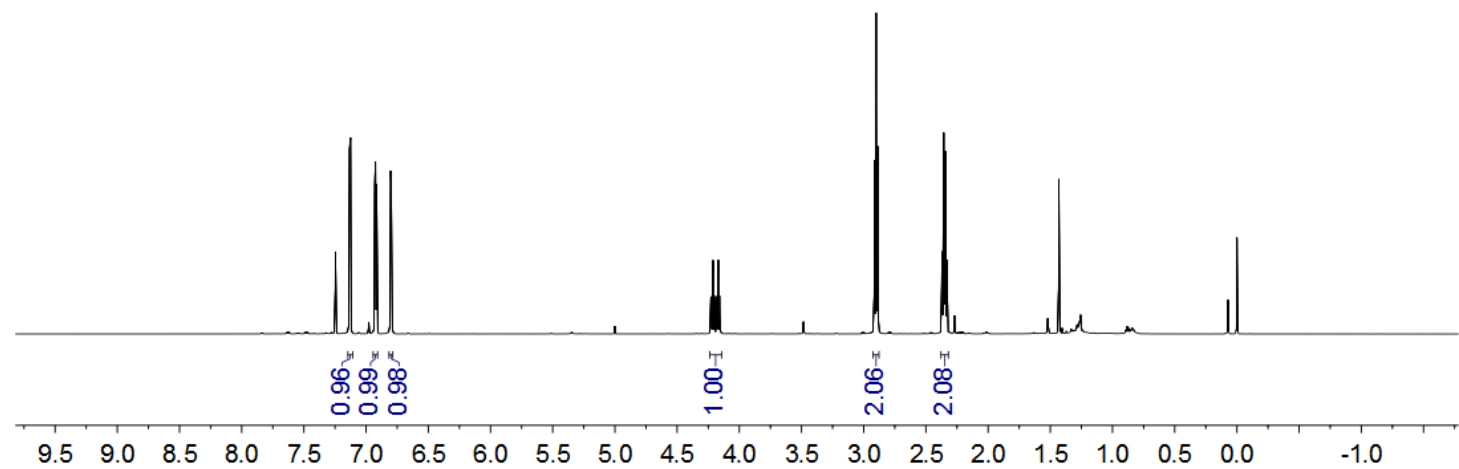

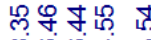

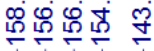

సलํำ

ถูํํำ
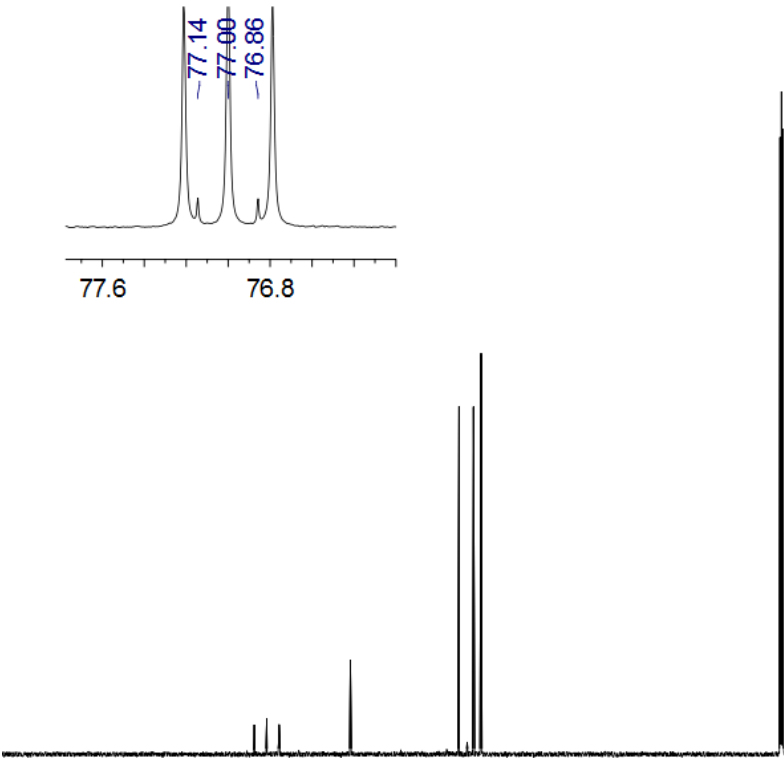

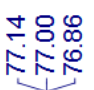

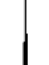

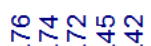

จูกิ่

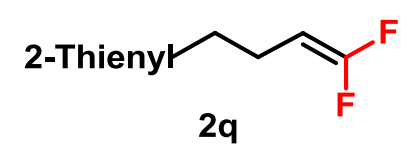

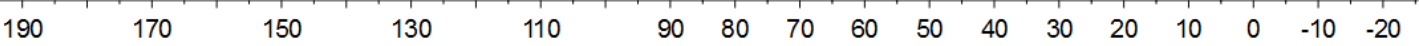




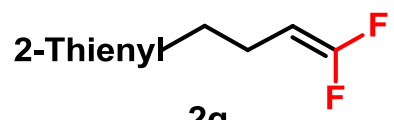

$\mathbf{2 q}$

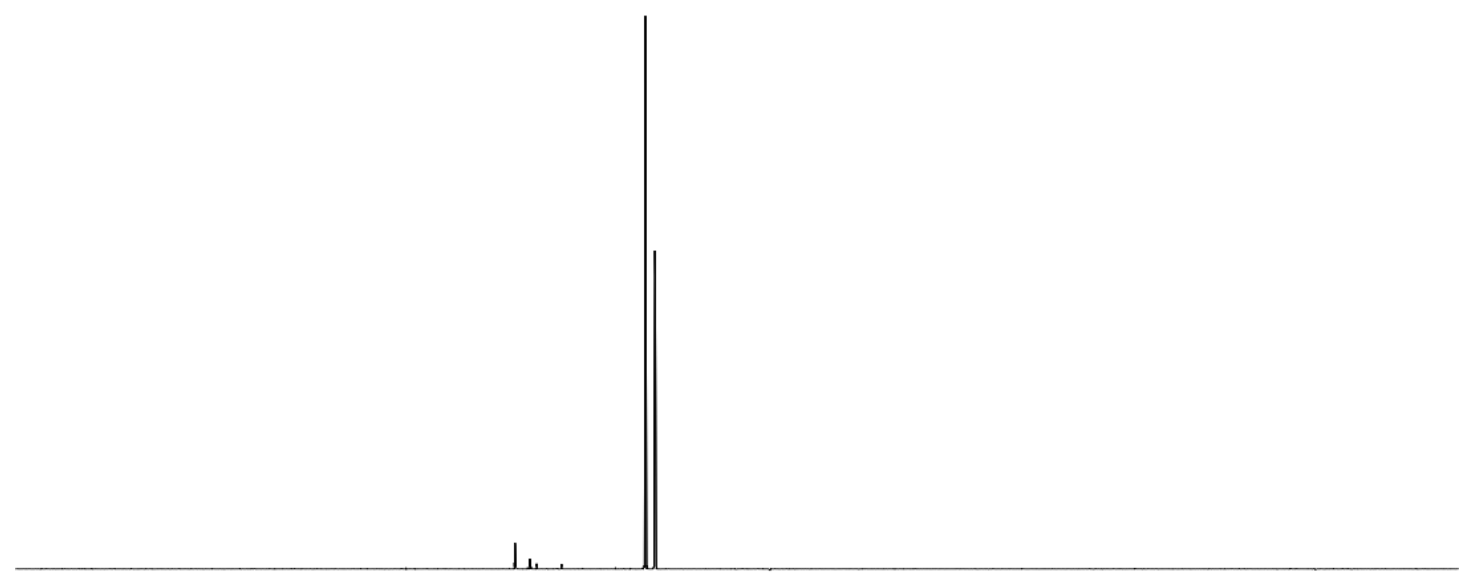

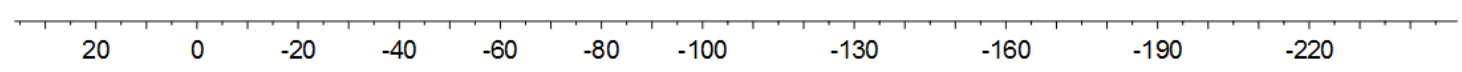

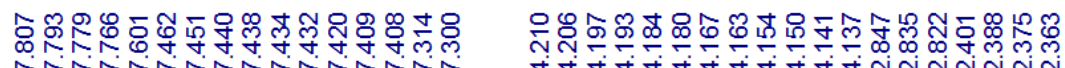
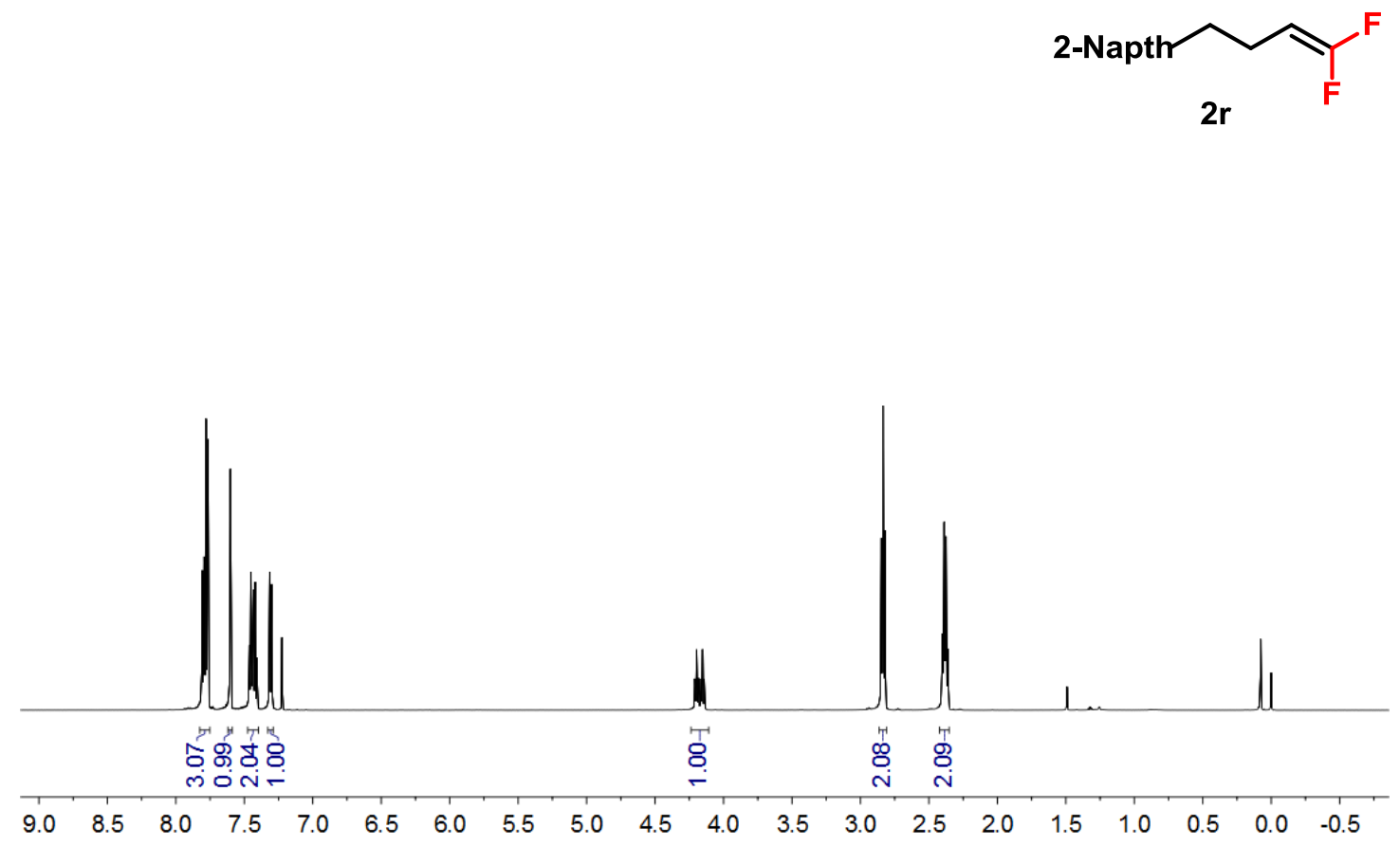


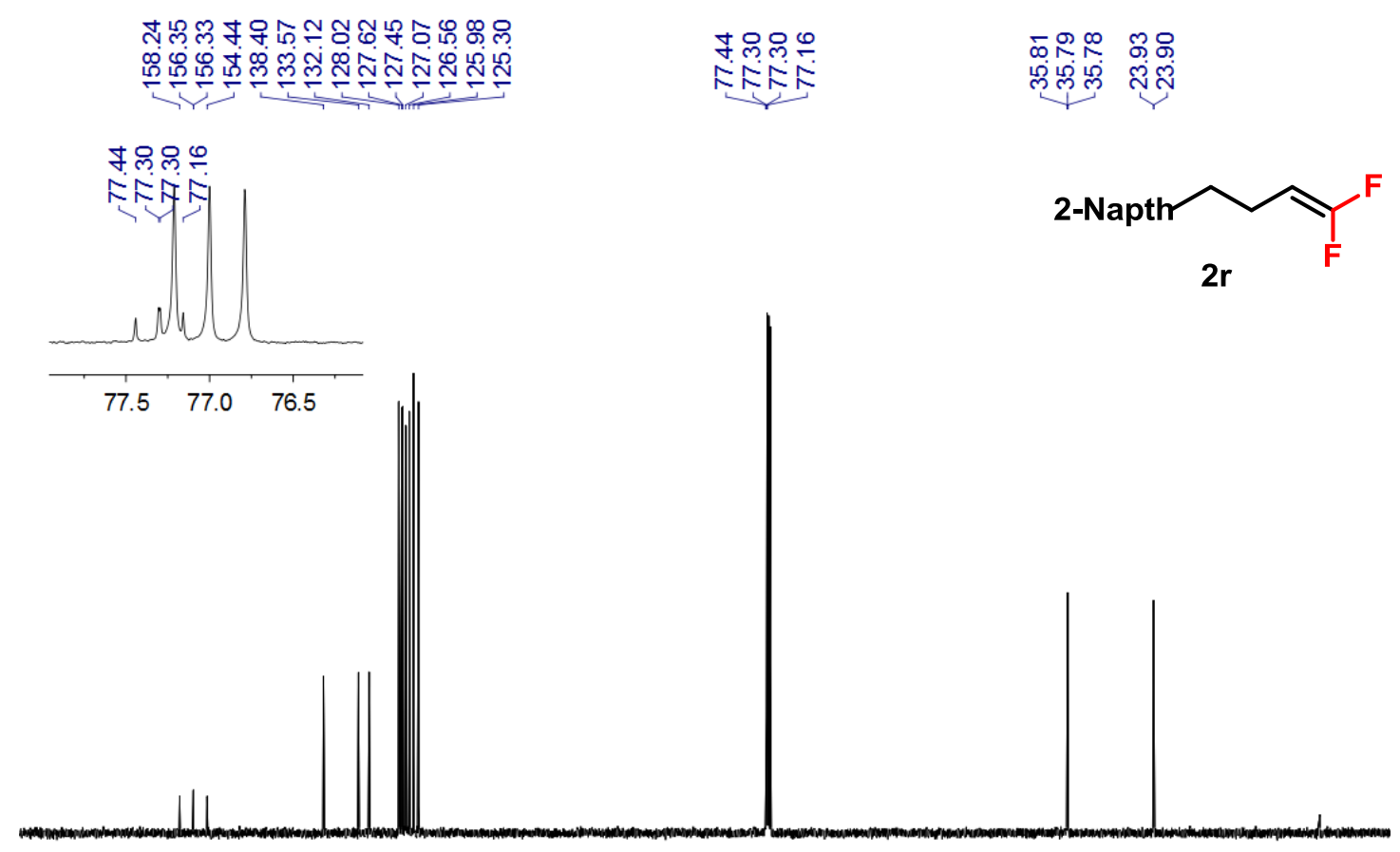

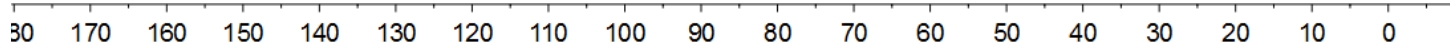

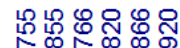

œ
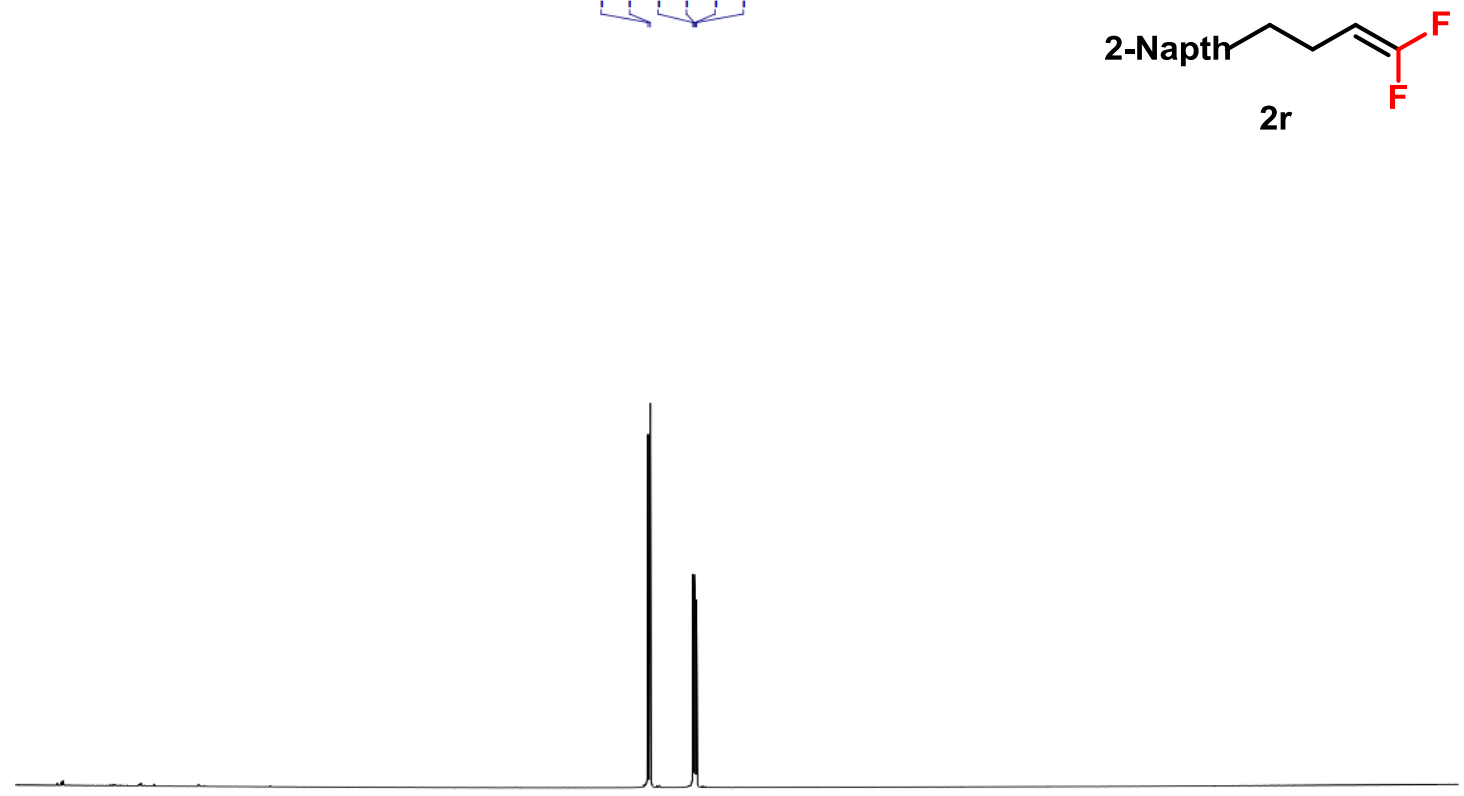

$-65 \quad-70 \quad-75$

$-80$

$-85$

$-90$

$-95$

$-100-105$

$-115$

$-120-1$ 


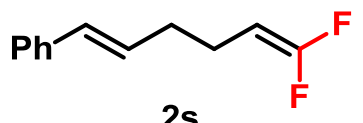

2s

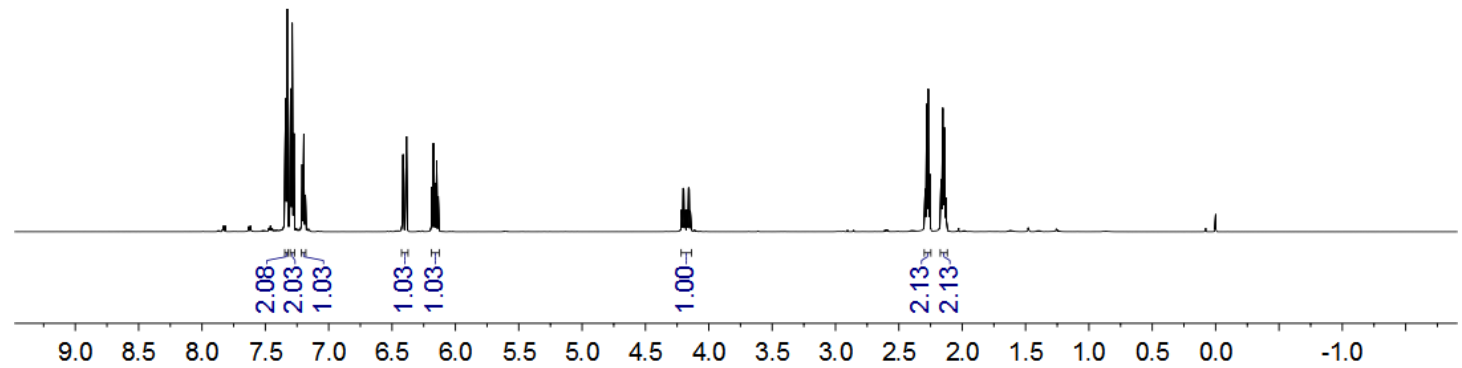

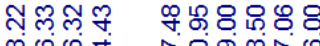

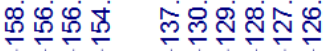

ใำำ

ลN
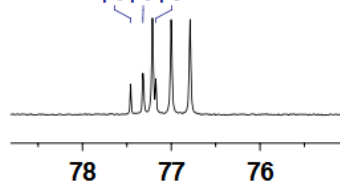

Q⿻日禸

KRE

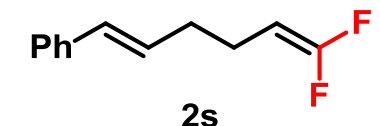

$2 s$

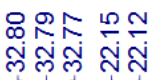

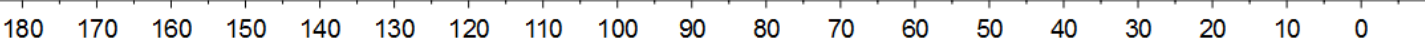




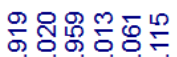

œ ळं कें के

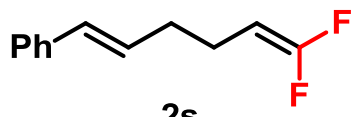

2s

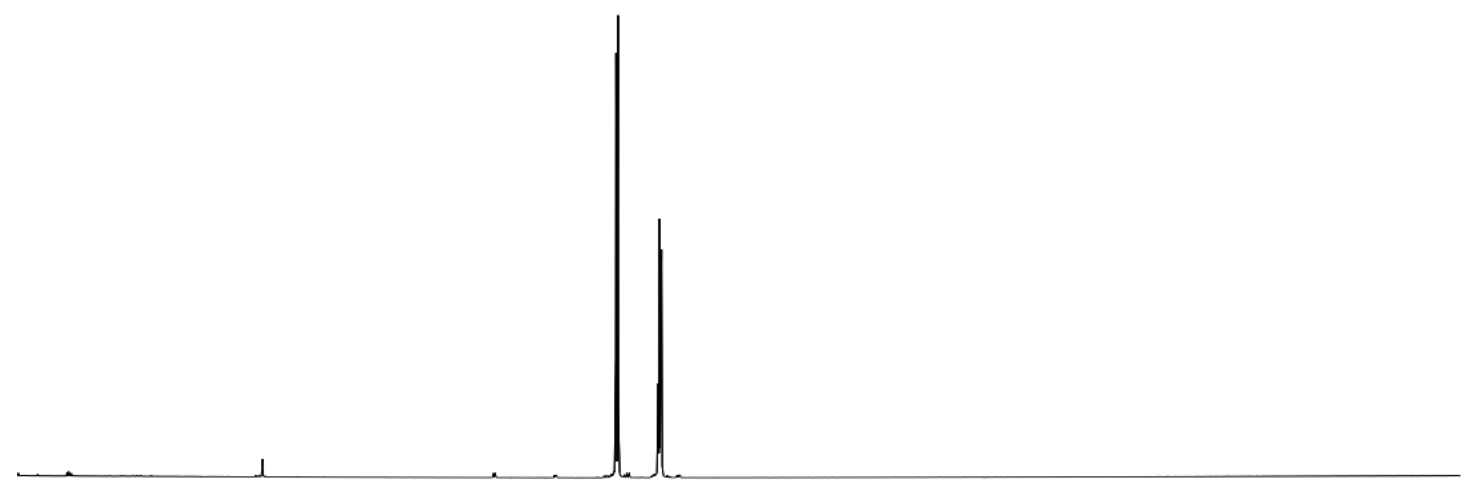

\begin{tabular}{llllllllll}
\hline 62 & -68 & -74 & -80 & -86 & -92 & -98 & -106 & -114 & -122
\end{tabular}

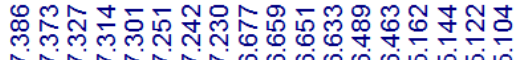

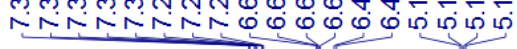
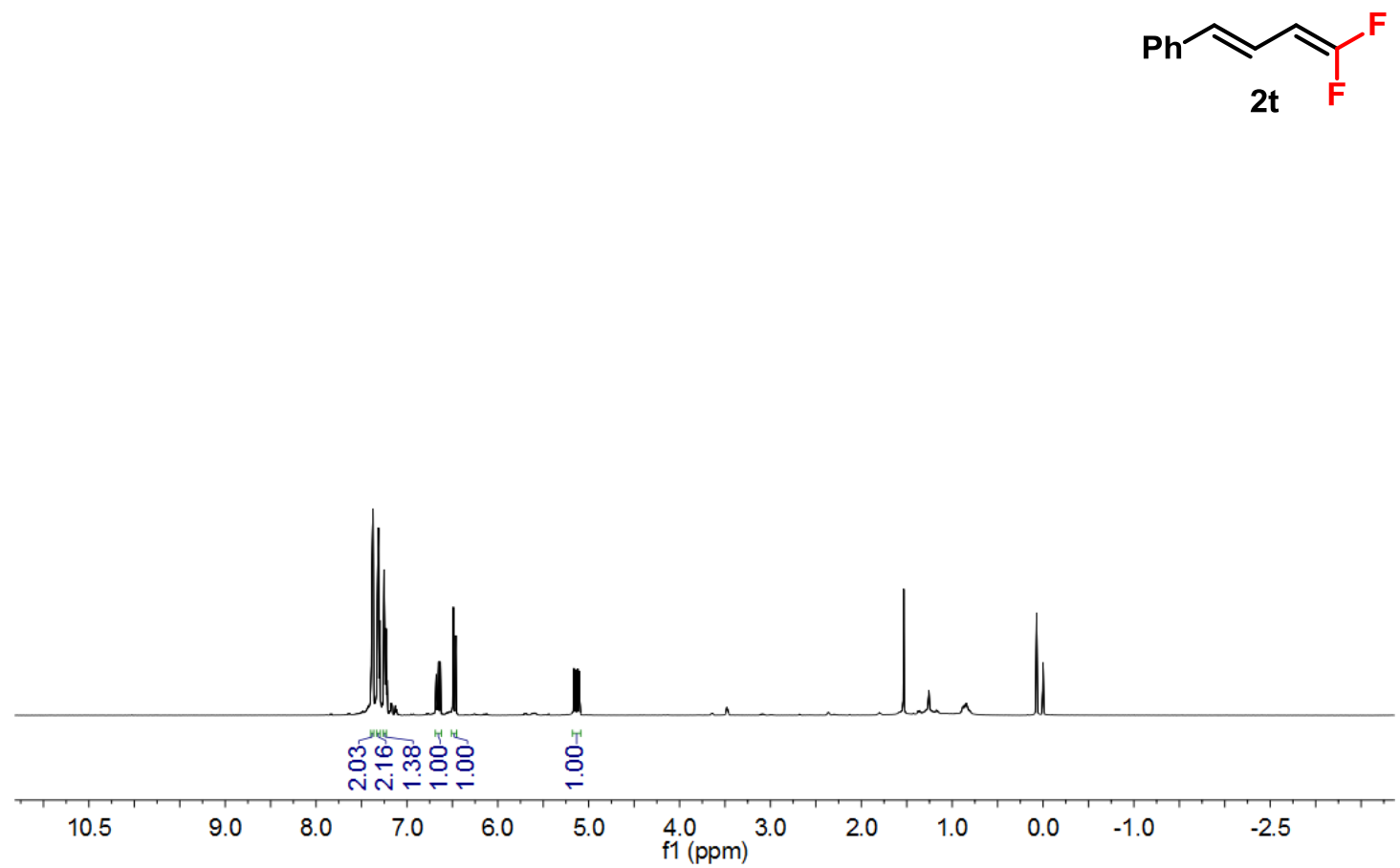

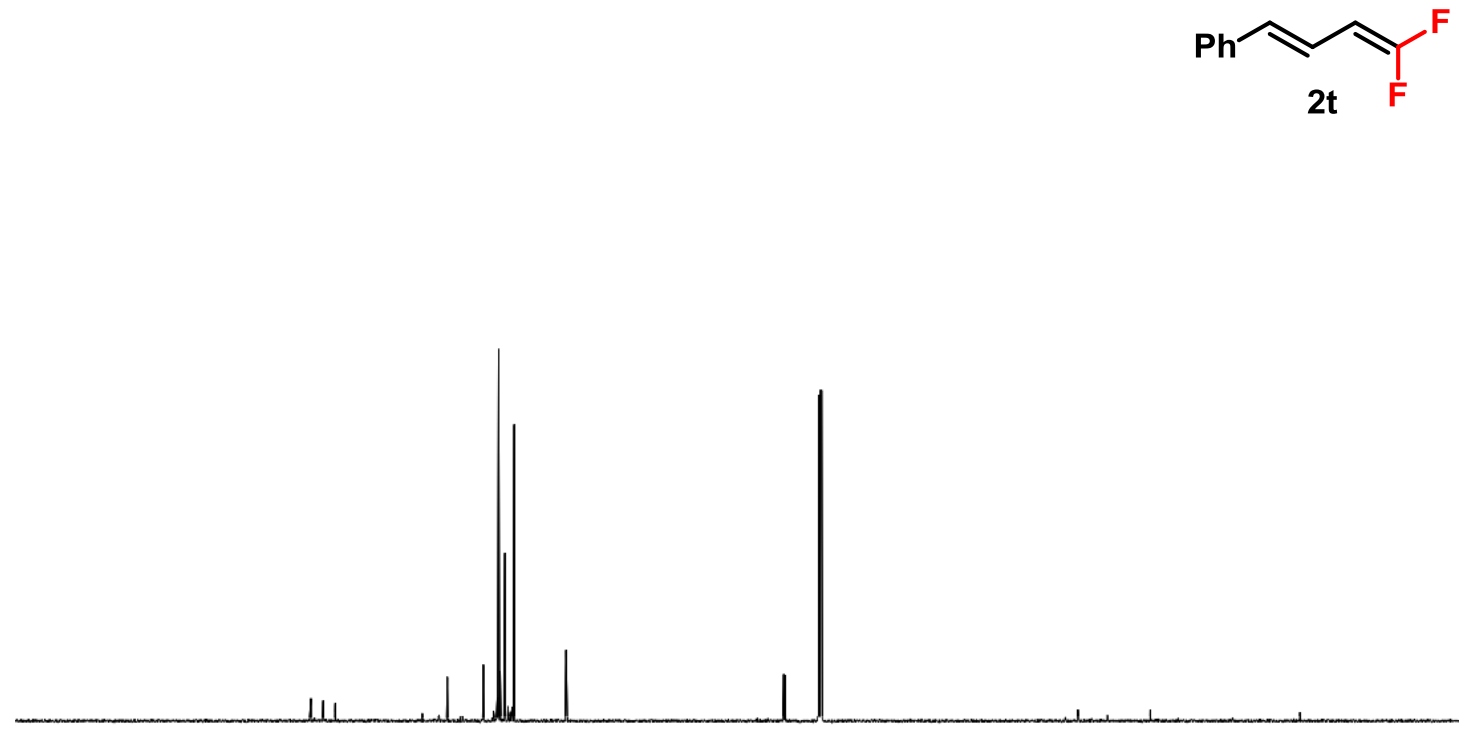

\begin{tabular}{lllllllllllllllll}
\hline 190 & 170 & 150 & 130 & 110 & 90 & 80 & 70 & 60 & 50 & 40 & 30 & 20 & 10 & 0 & -10 & $\mathrm{f} 1(\mathrm{ppm})$
\end{tabular}

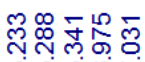

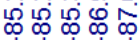
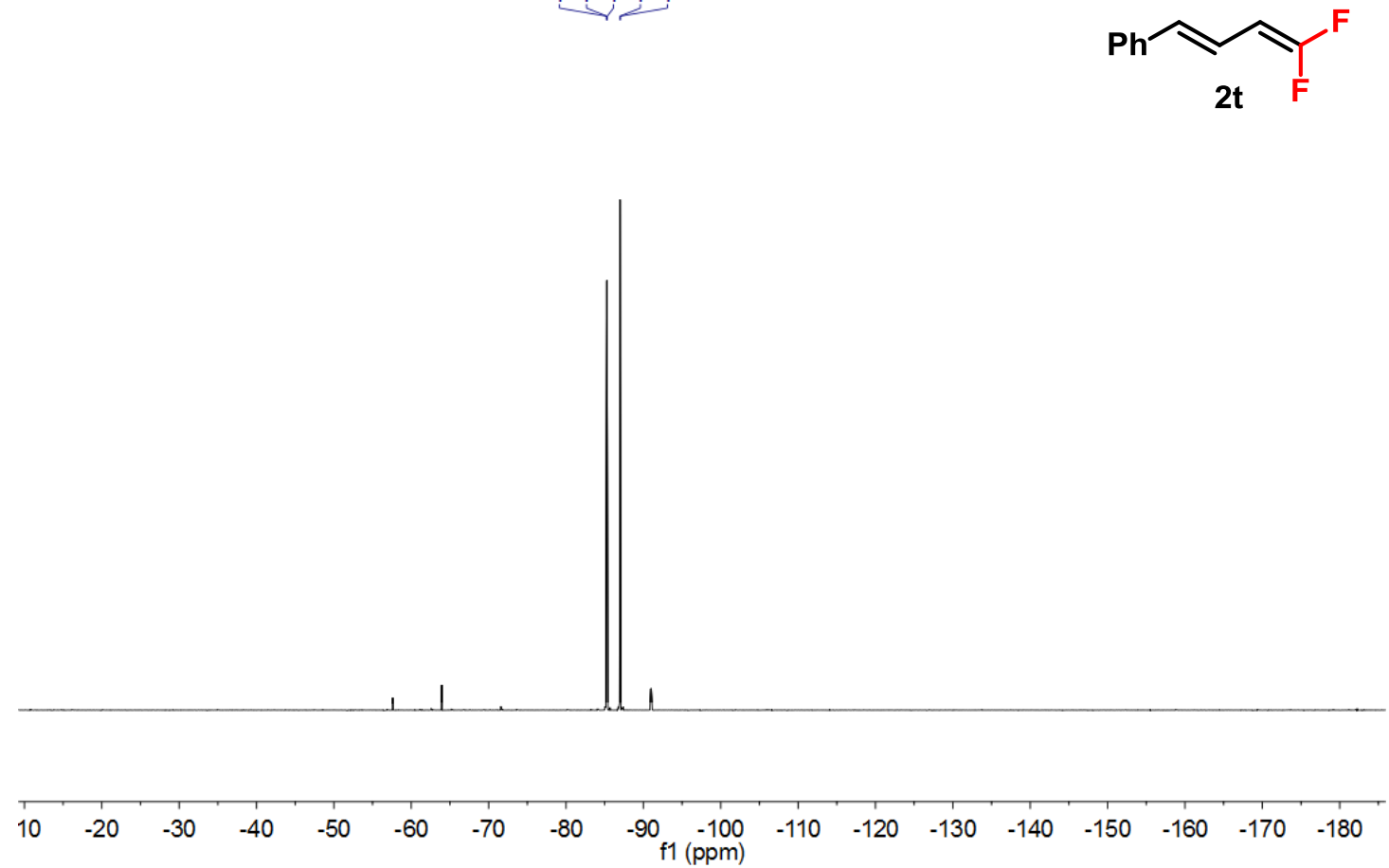

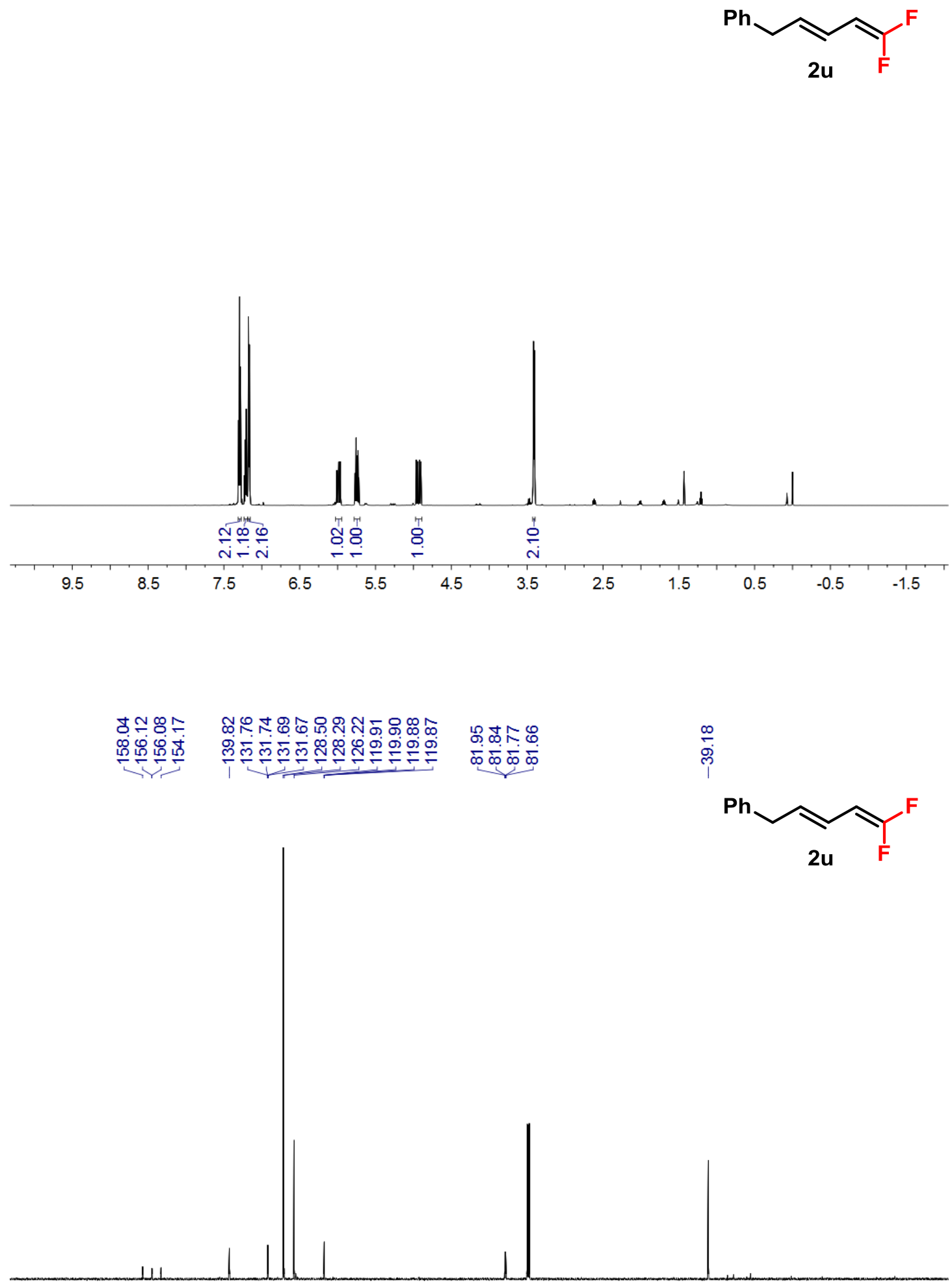

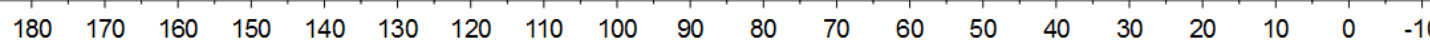



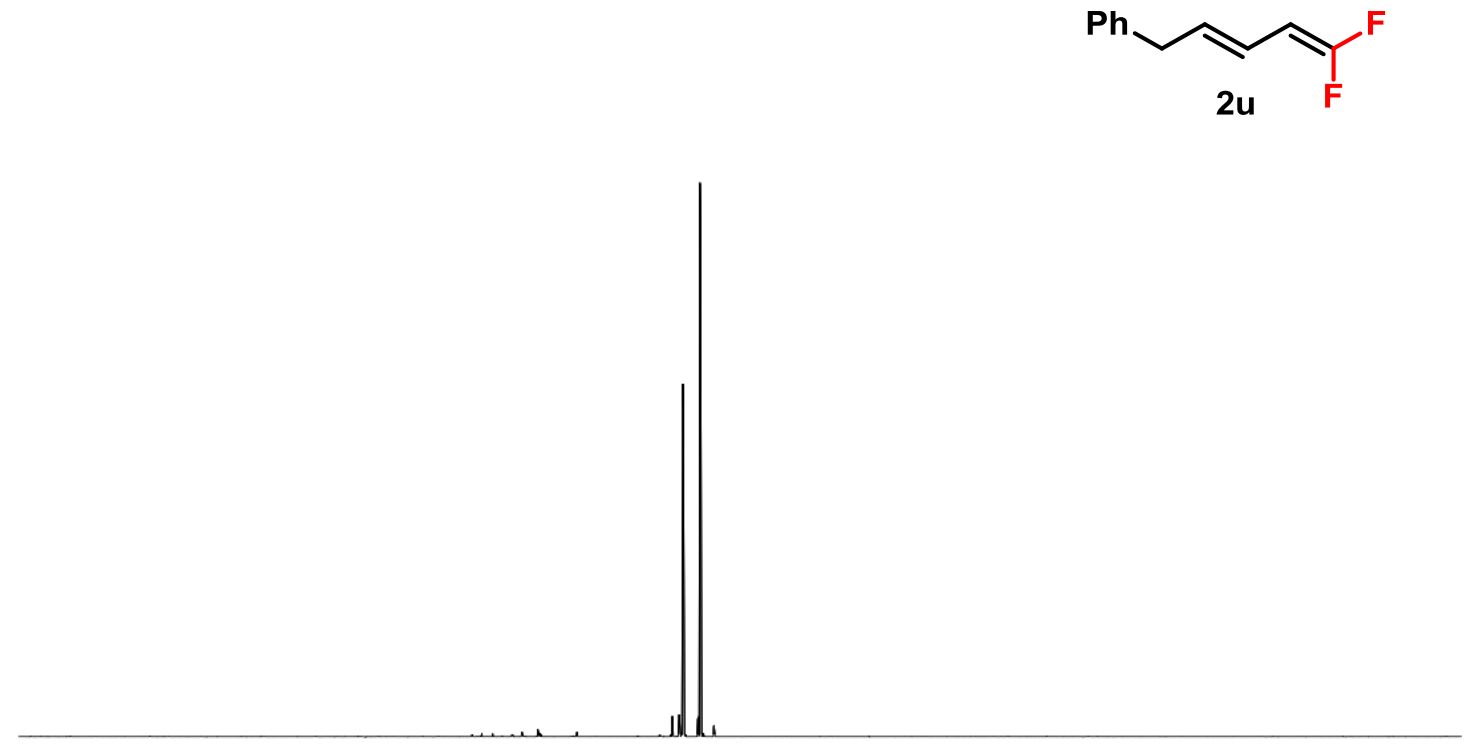

\begin{tabular}{llllllllllllllll}
\hline 0 & -10 & -20 & -30 & -40 & -50 & -60 & -70 & -80 & -90 & -100 & -120 & -140 & -160 & -180 & 1 \\
\hline
\end{tabular}

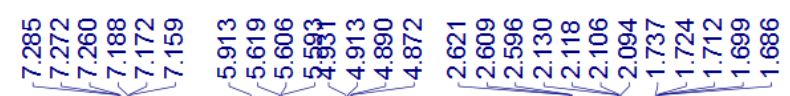
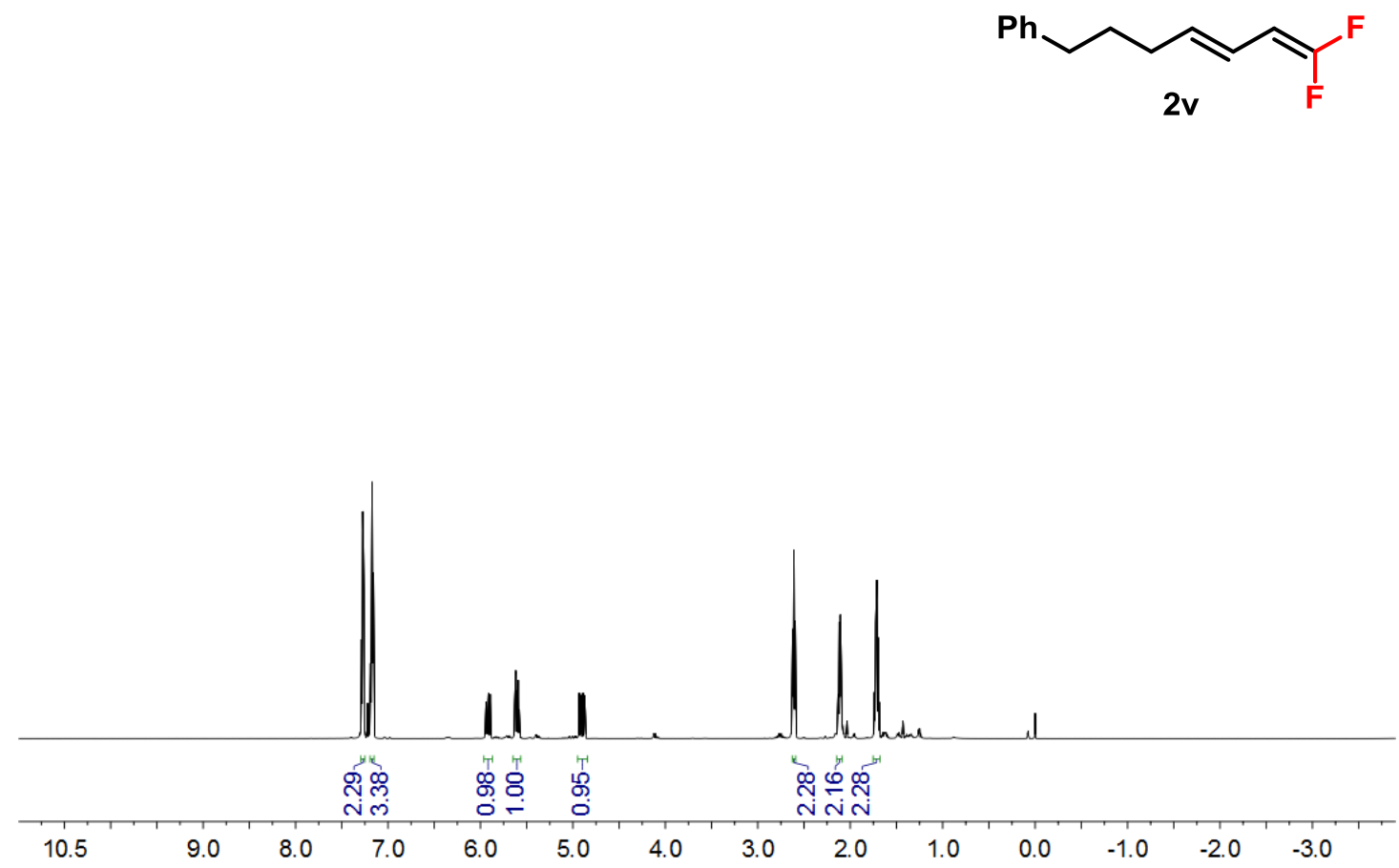


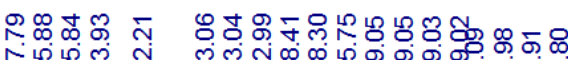

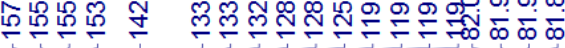

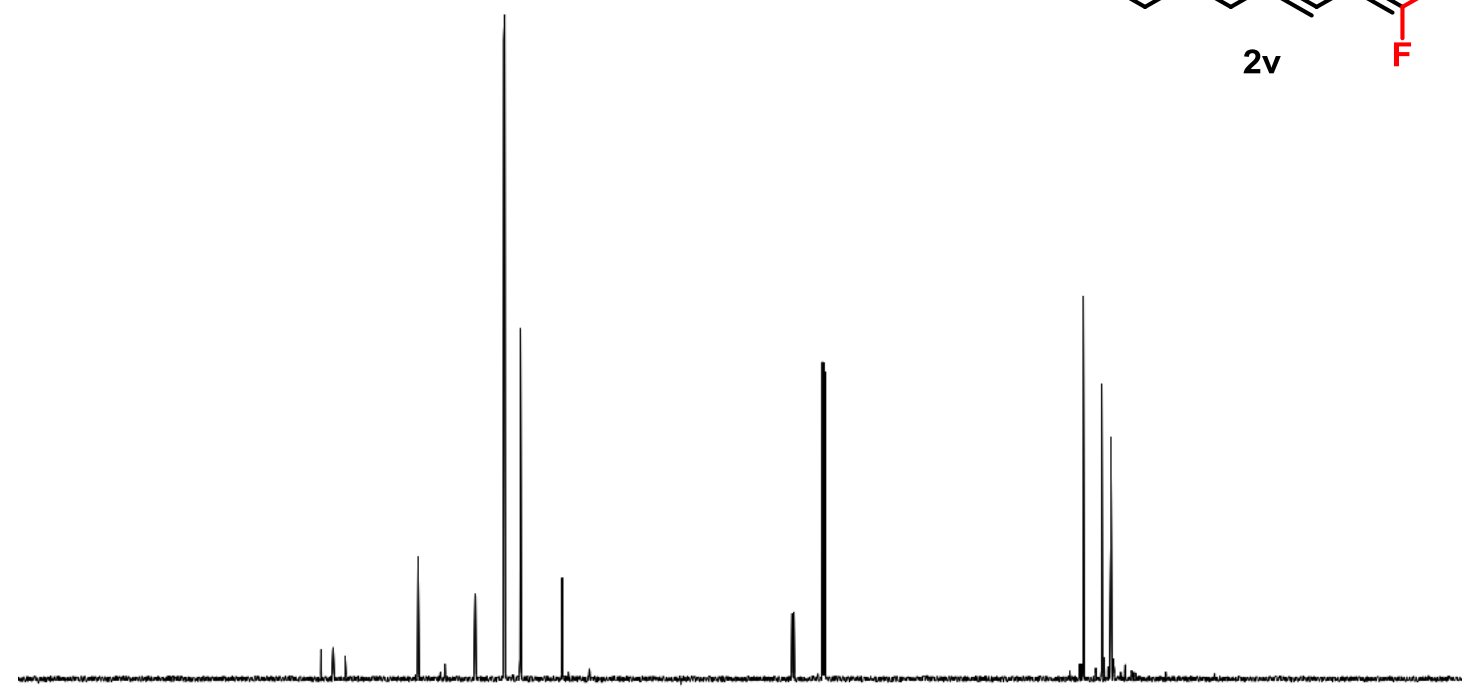

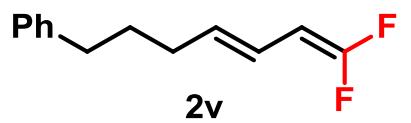

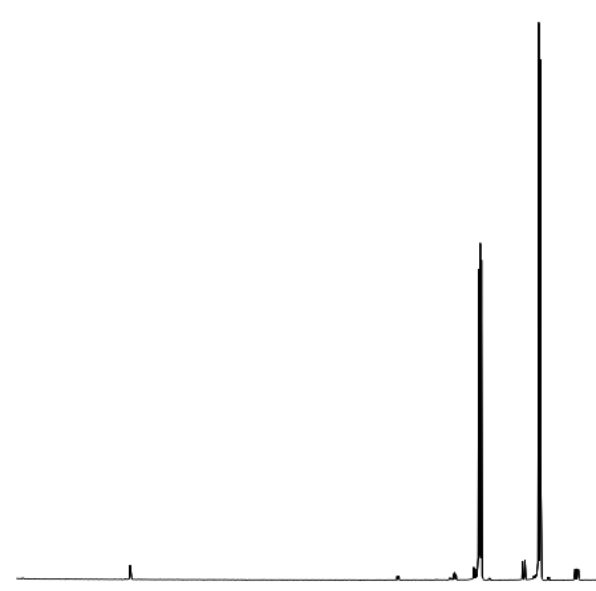

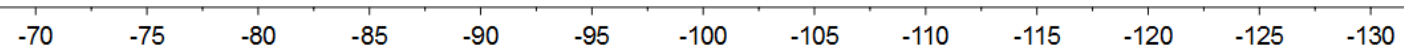



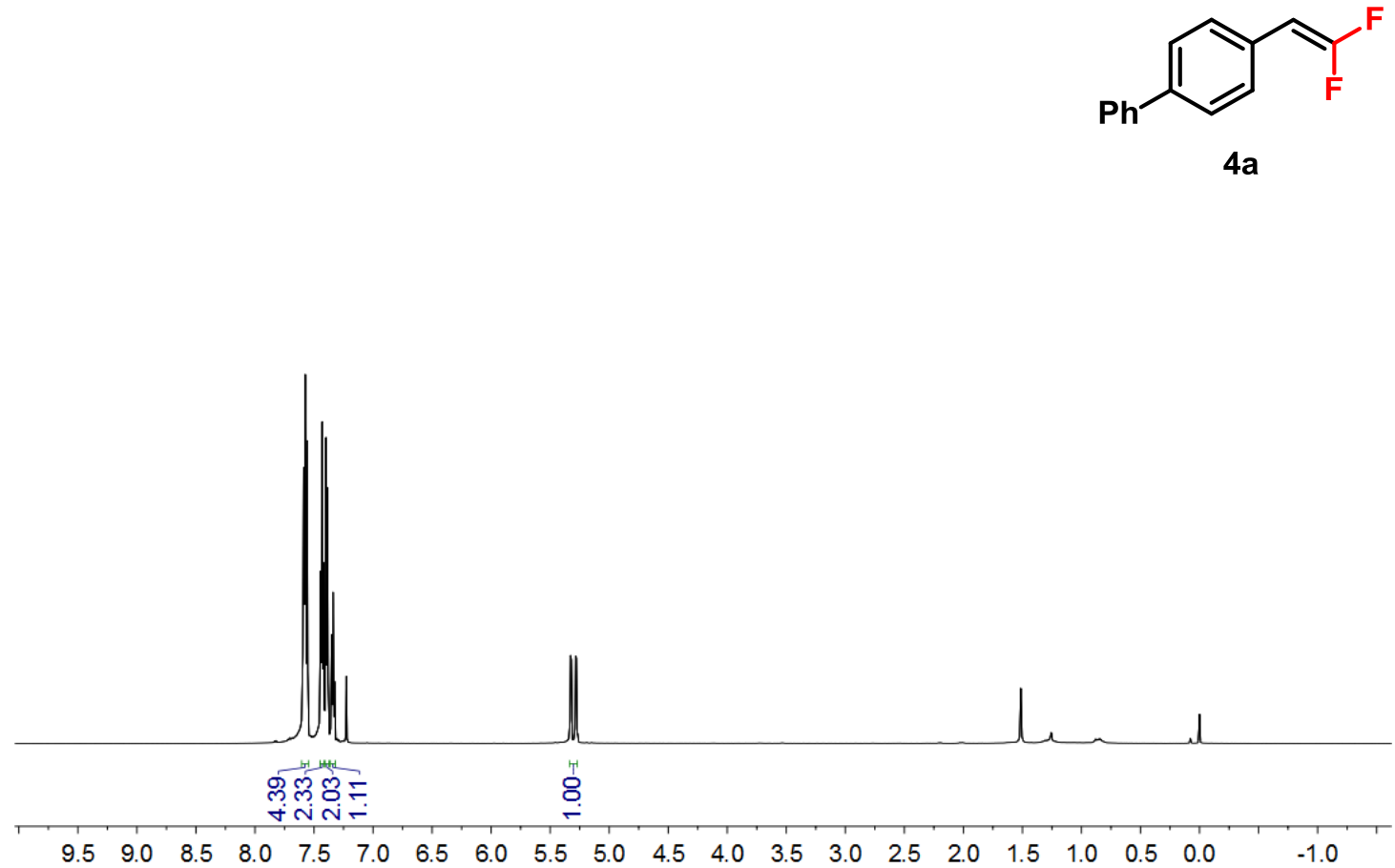

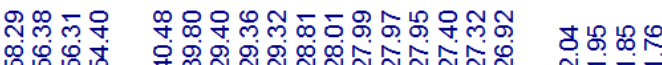

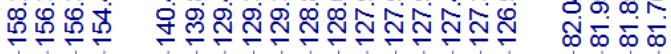<smiles>FC(F)=Cc1ccc(-c2ccccc2)cc1</smiles>

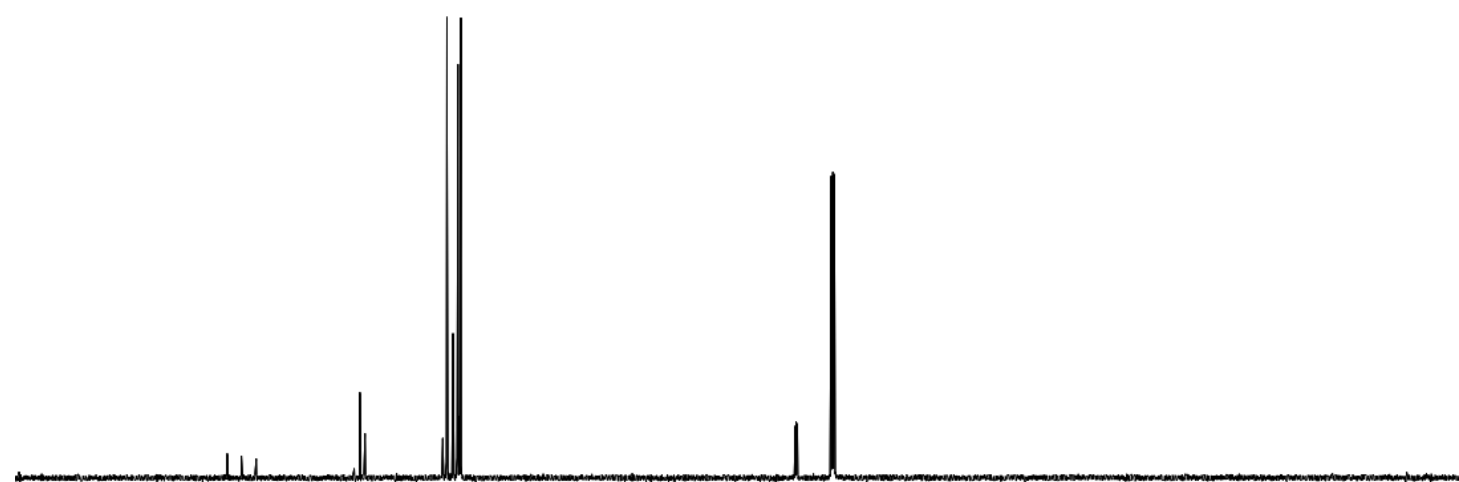

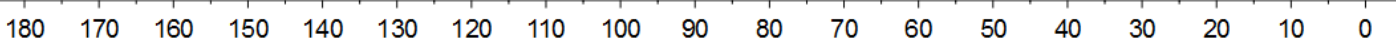




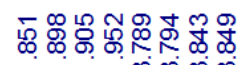

-<smiles>FC(F)=Cc1ccc(-c2ccccc2)cc1</smiles>

4a

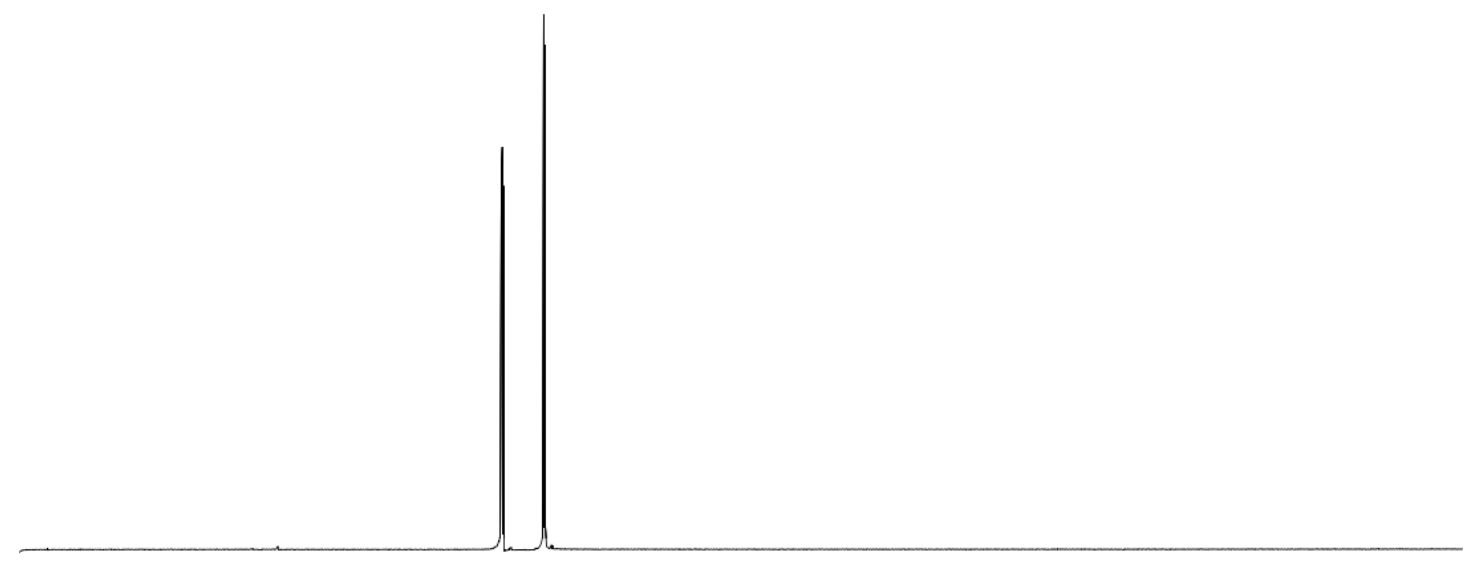

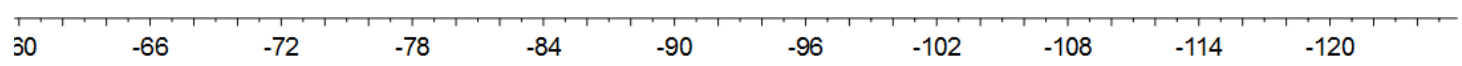

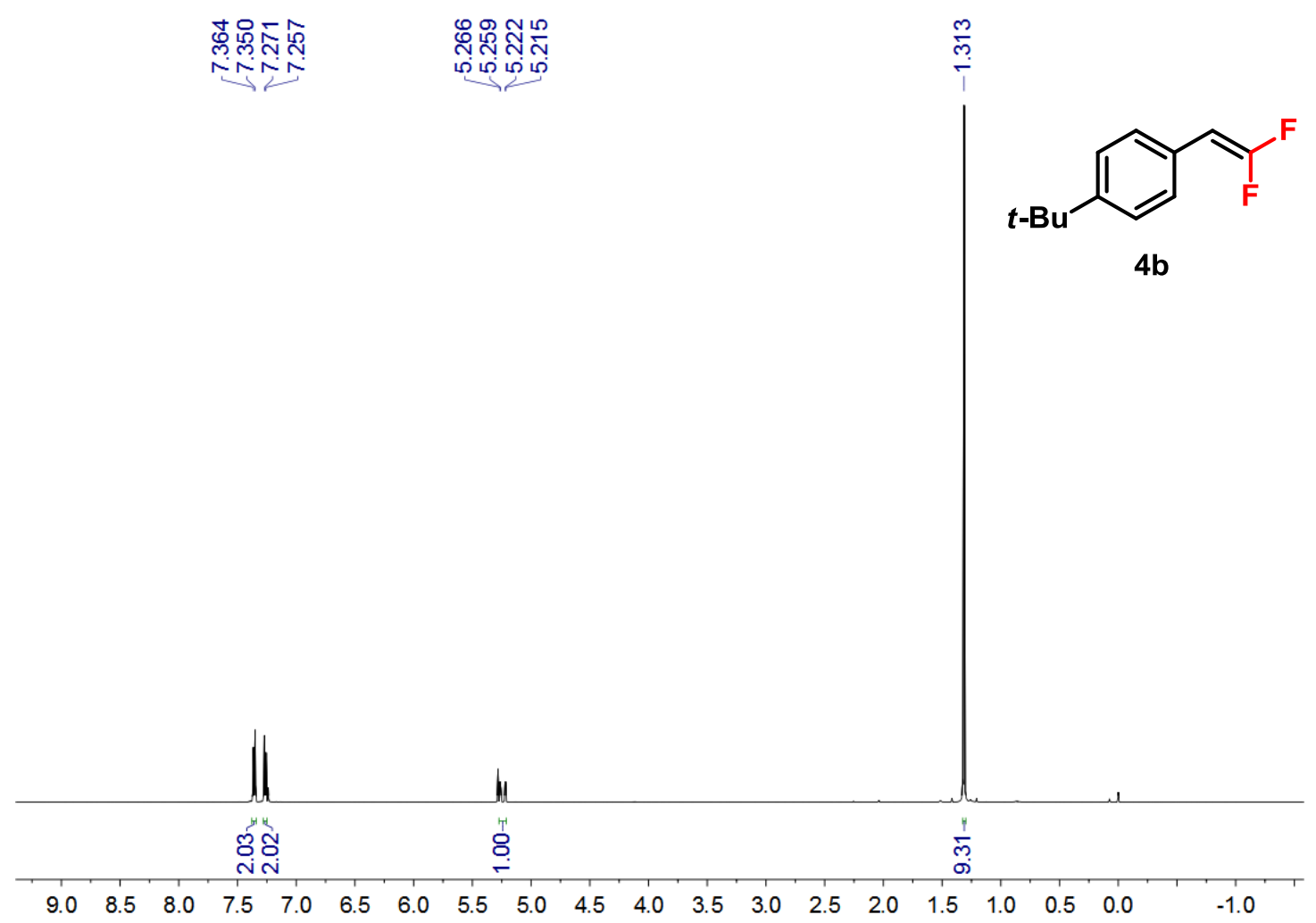




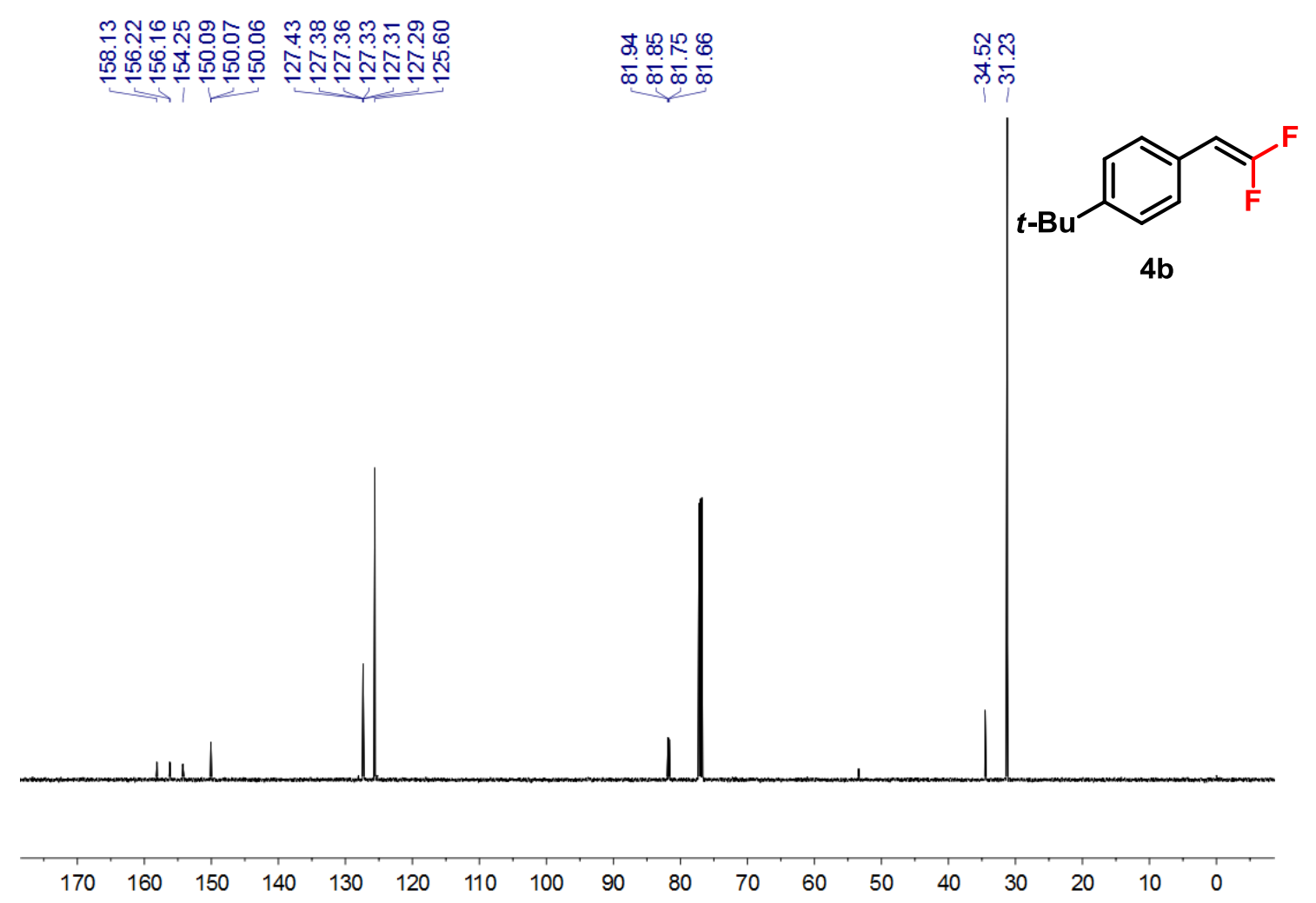

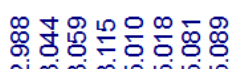

लिळ

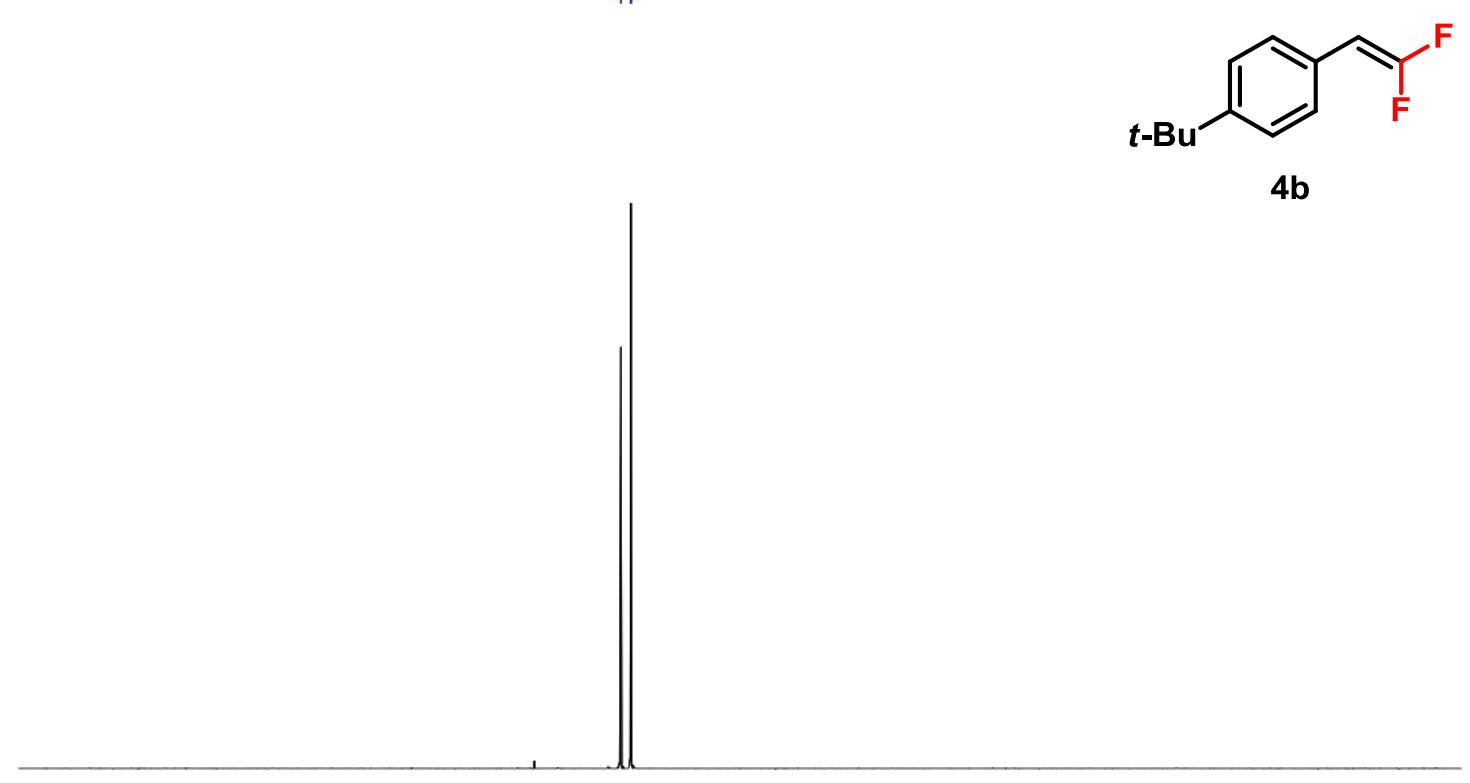

\begin{tabular}{lllllllllll}
\hline & 0 & -20 & -40 & -60 & -80 & -100 & -130 & -160 & -190 & -220
\end{tabular}



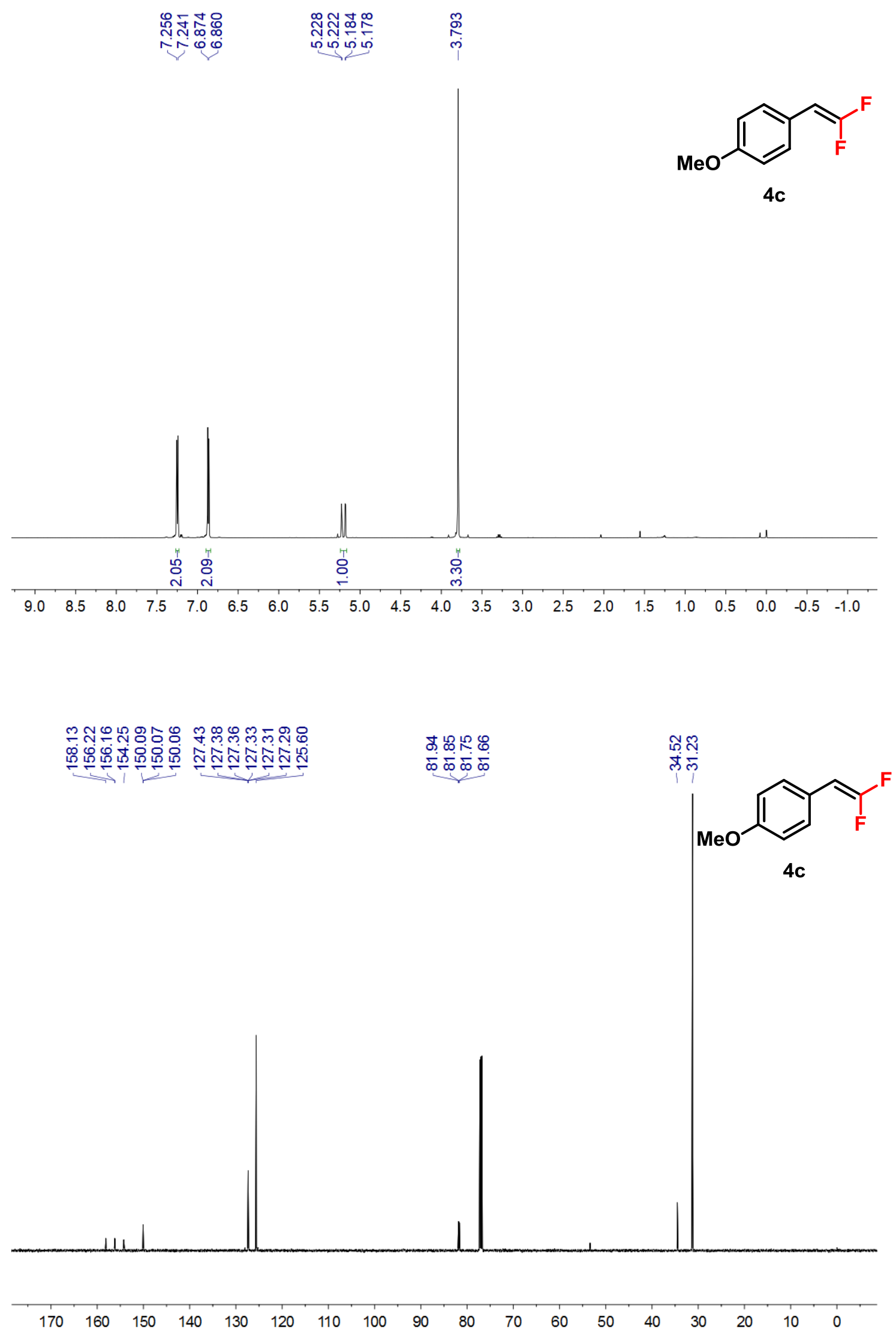

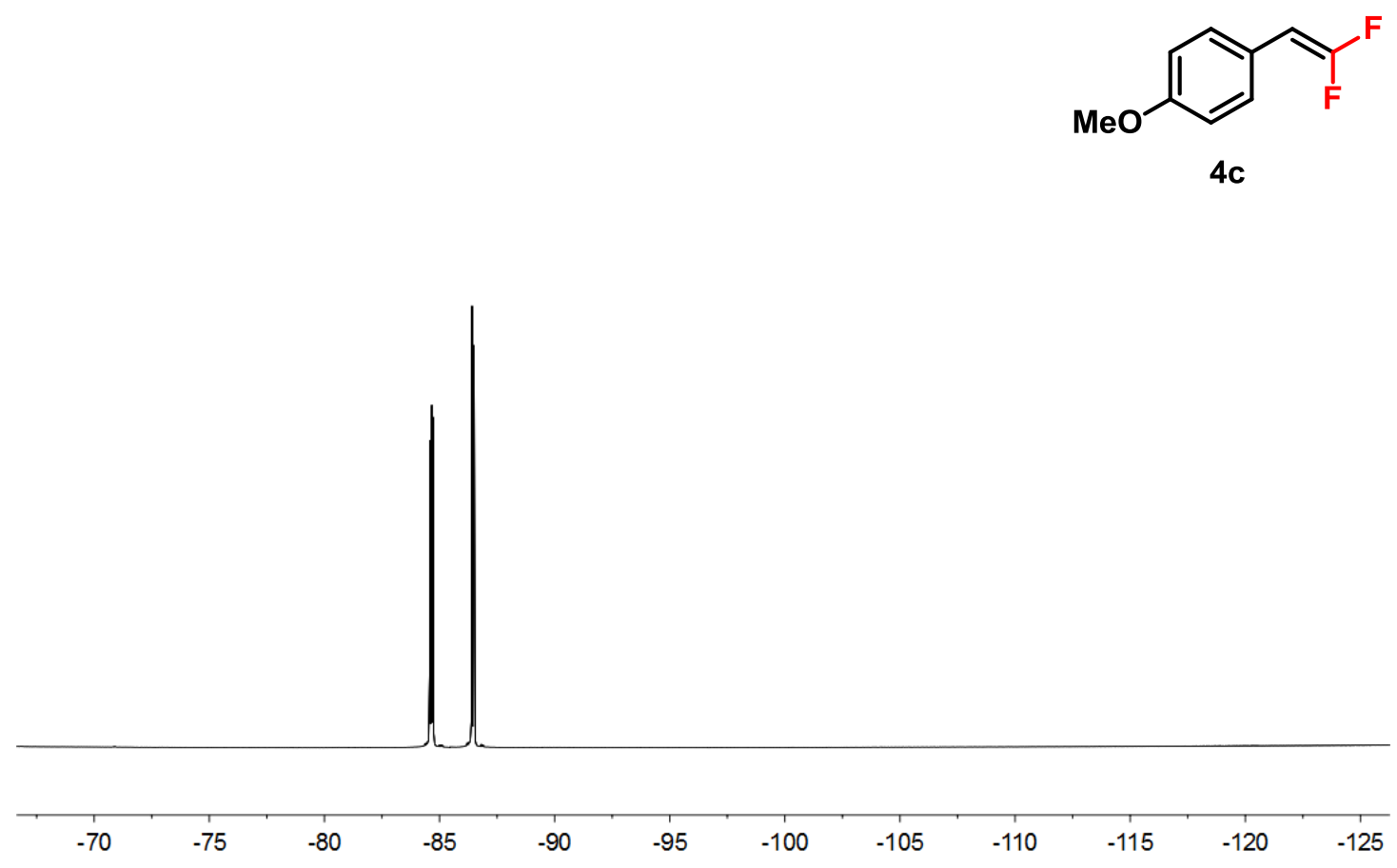

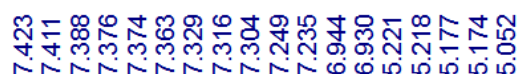

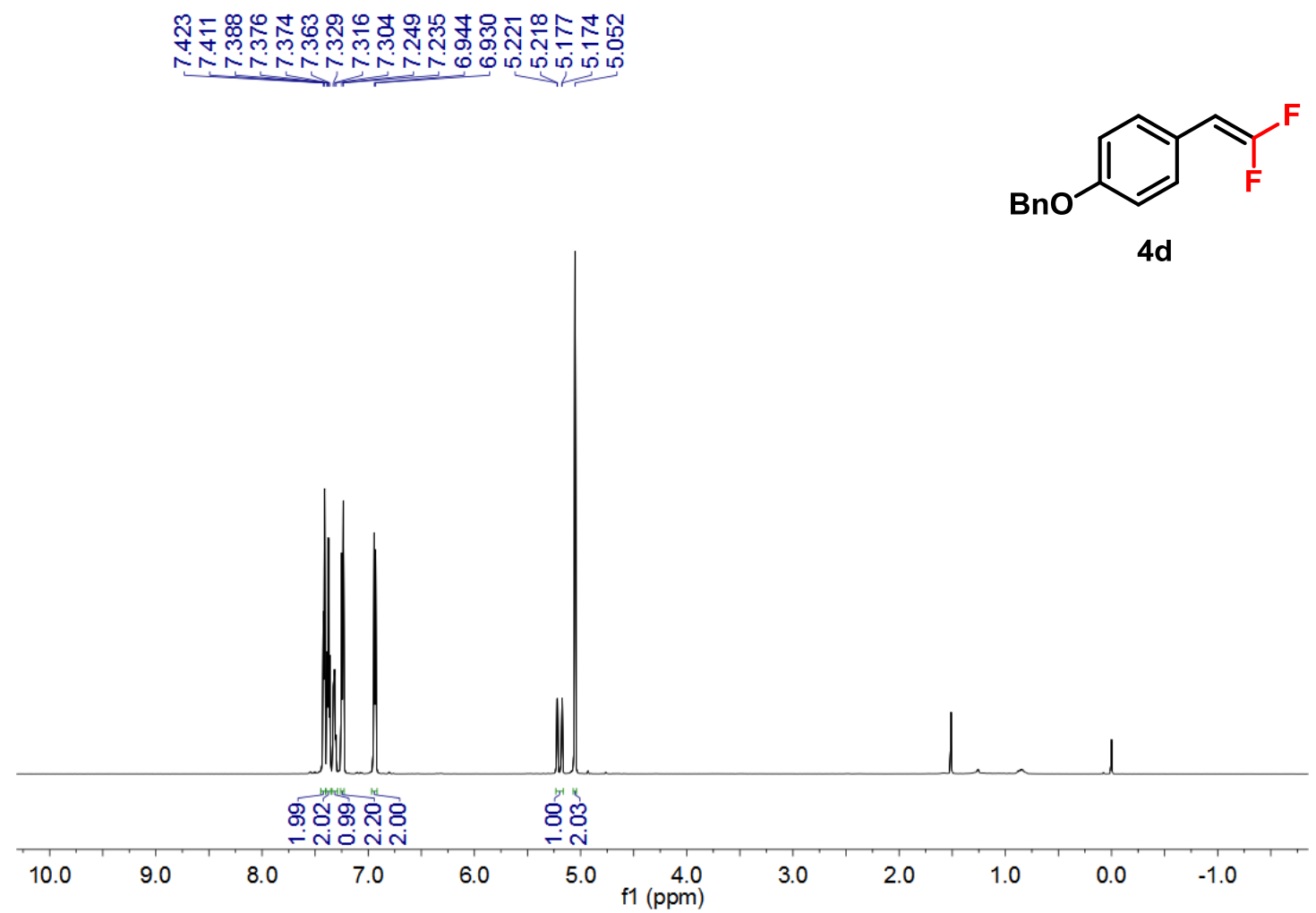




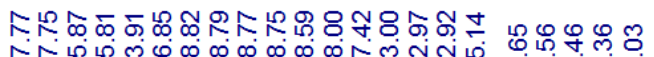

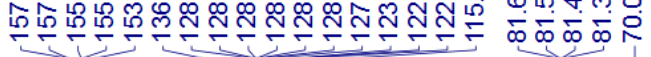

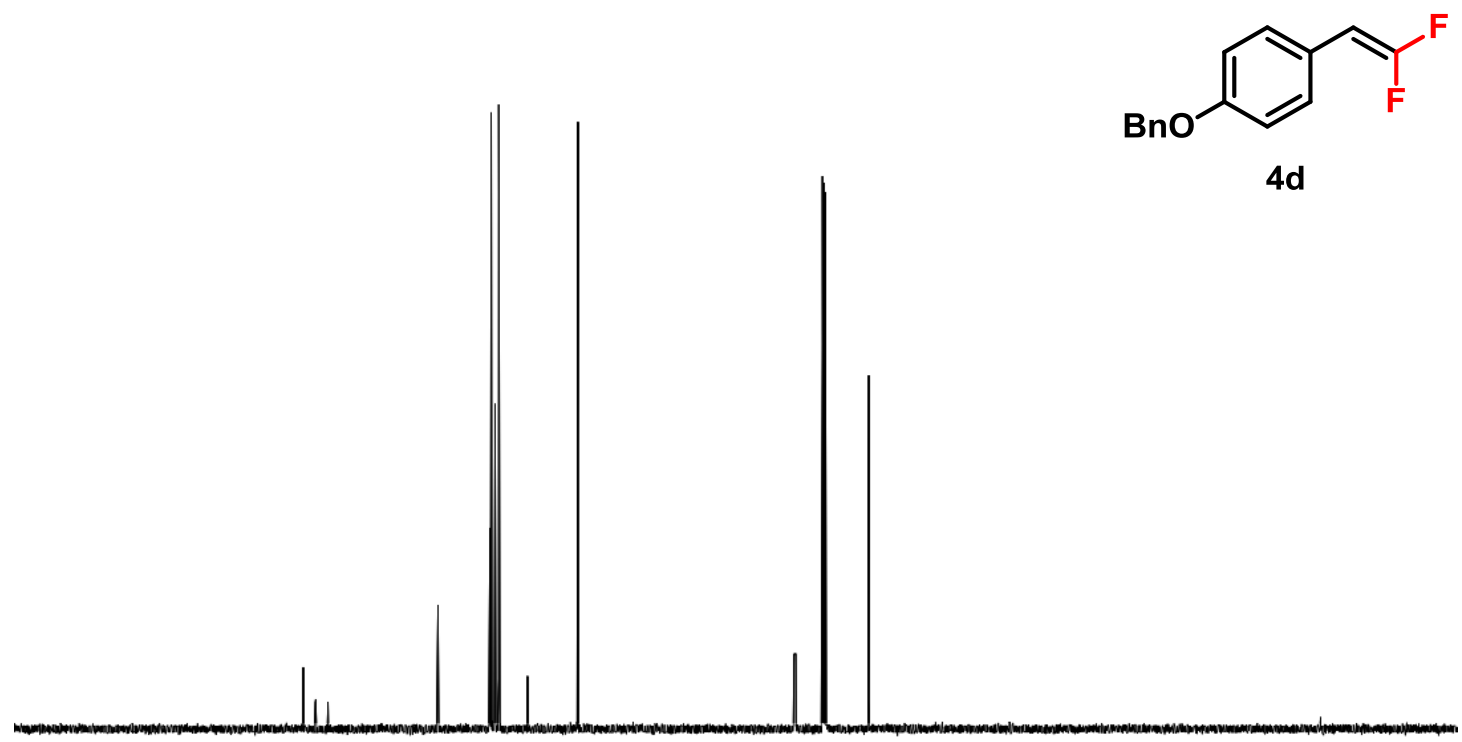

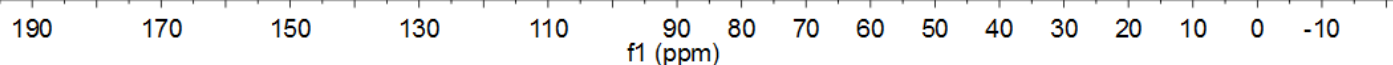

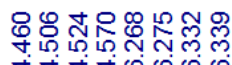

एक<smiles>O=C(Oc1ccc(C=C(F)F)cc1)c1ccccc1</smiles>

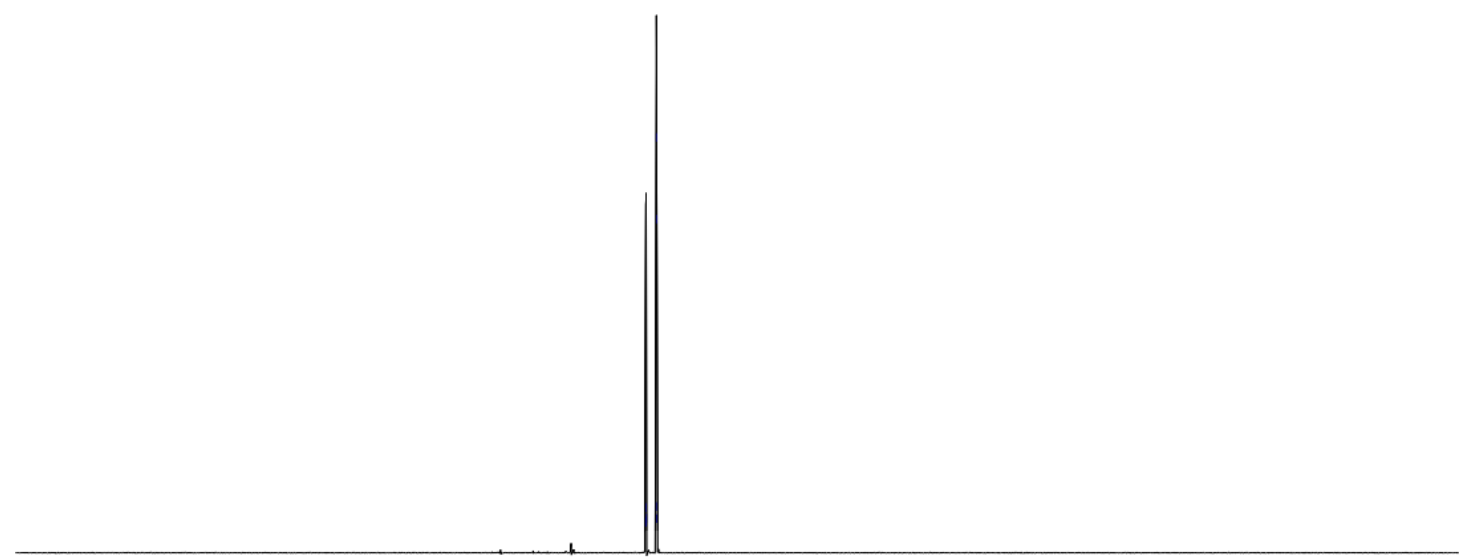

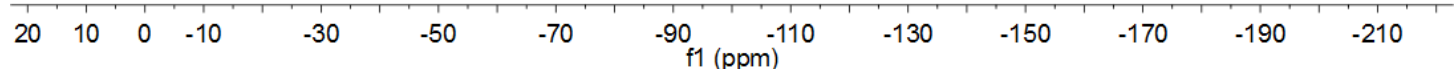




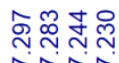

NNN

员专欲

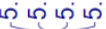
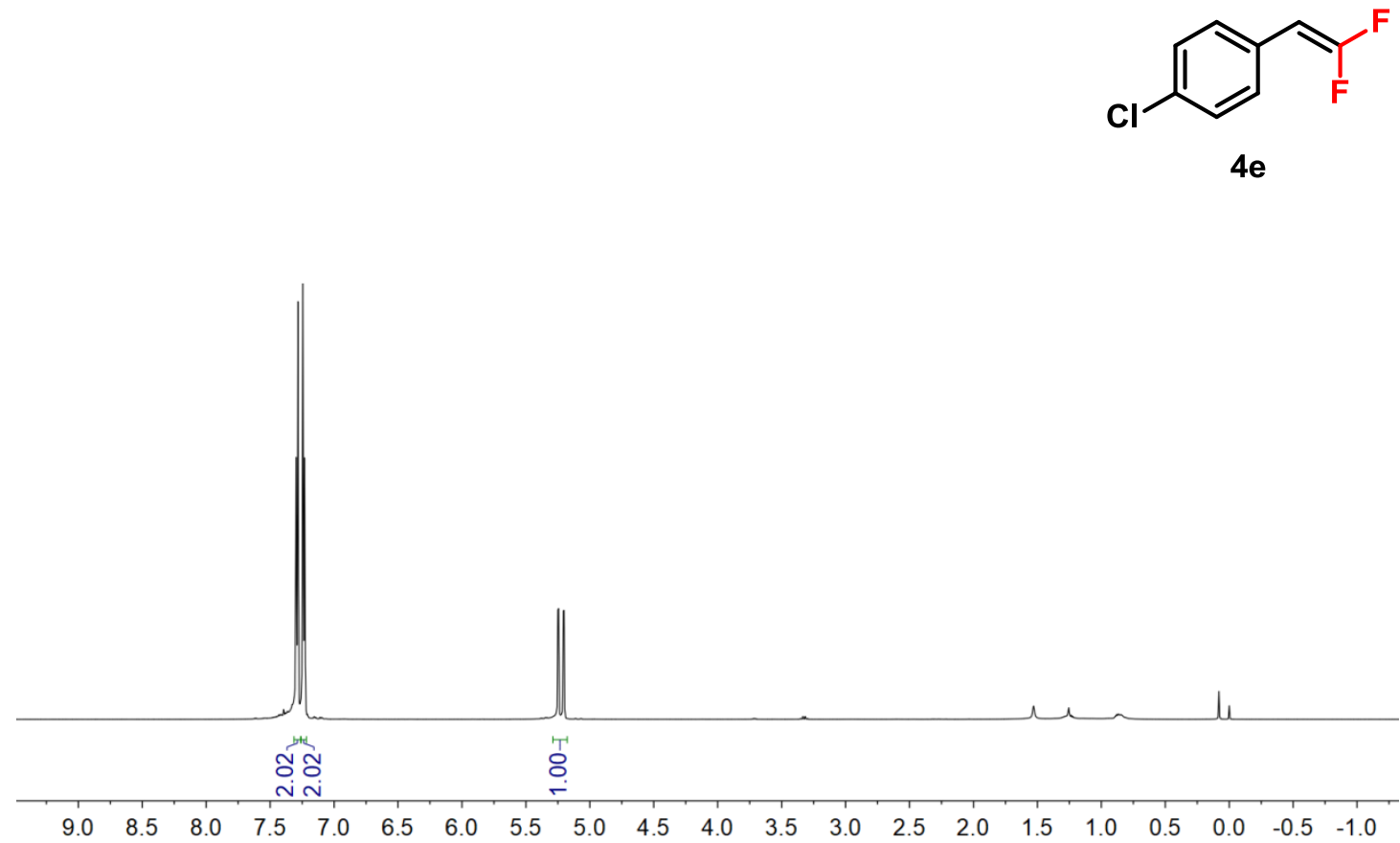

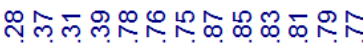

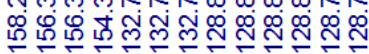

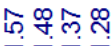

$\bar{\infty} \dot{\infty} \bar{\infty}$

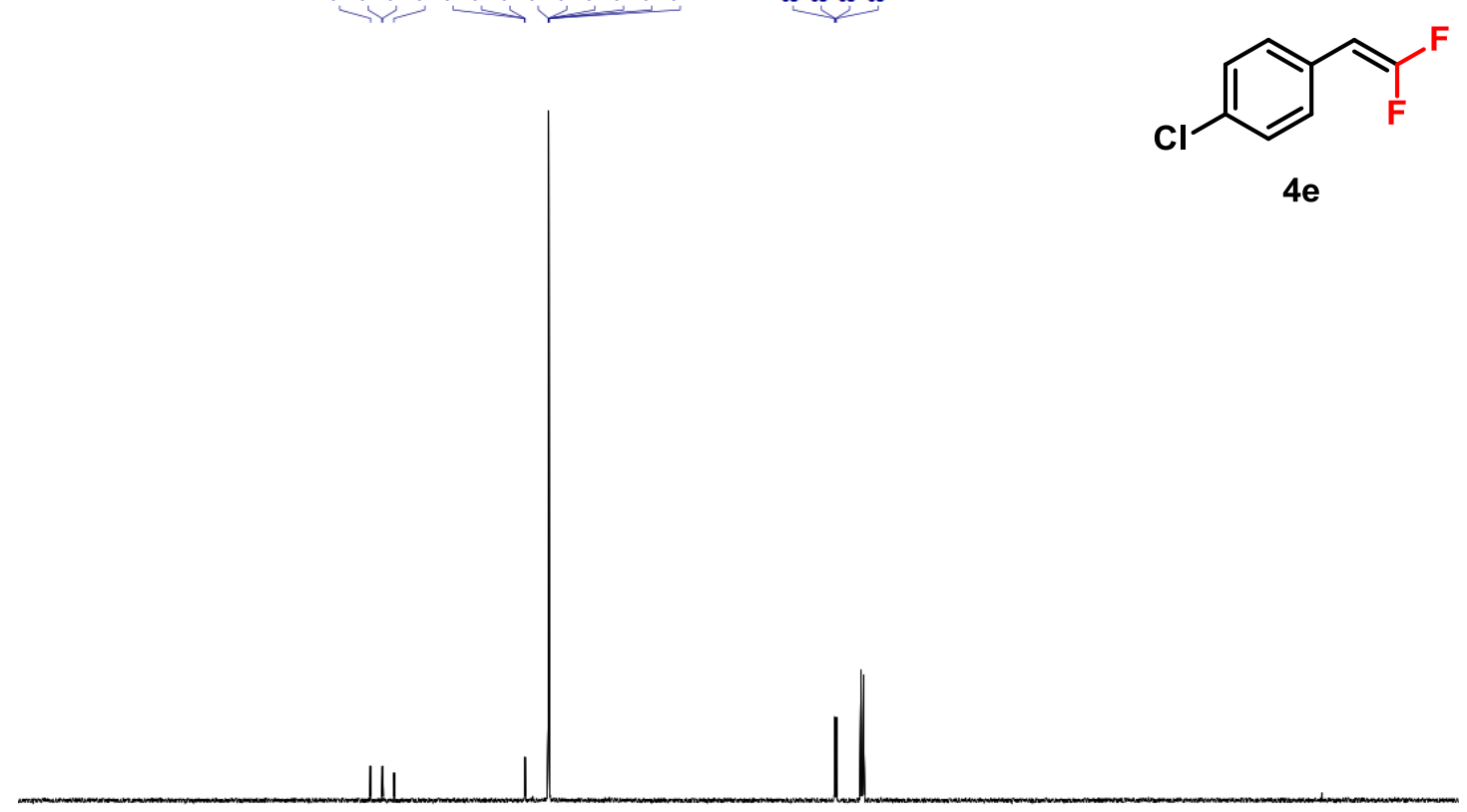

$210 \quad 190$

170 


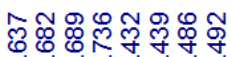

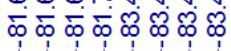<smiles>FC(F)=Cc1ccc(Cl)cc1</smiles>

$4 e$

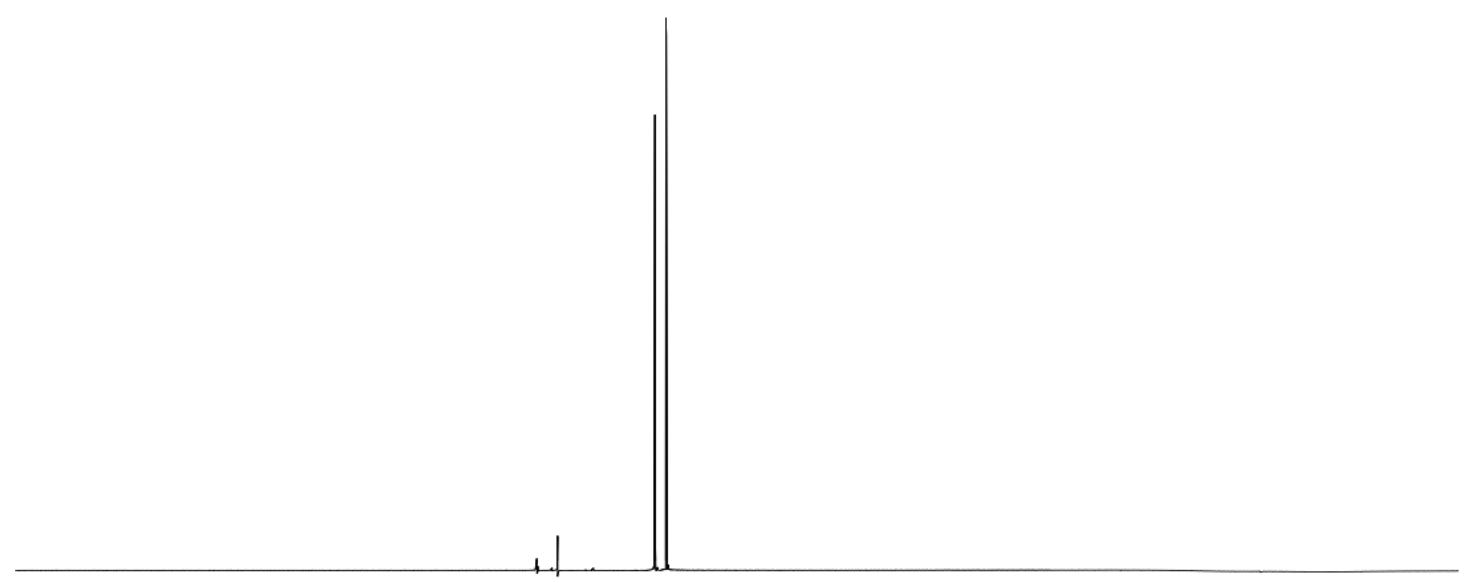

$\begin{array}{lllllllllllllllllll}20 & 10 & 0 & -10 & -20 & -30 & -40 & -50 & -60 & -70 & -80 & -90 & -100 & -120 & -140 & -160 & -180 & -200\end{array}$

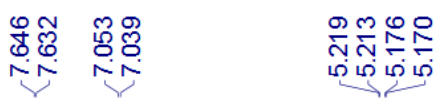

$\overbrace{4 f}^{P}$

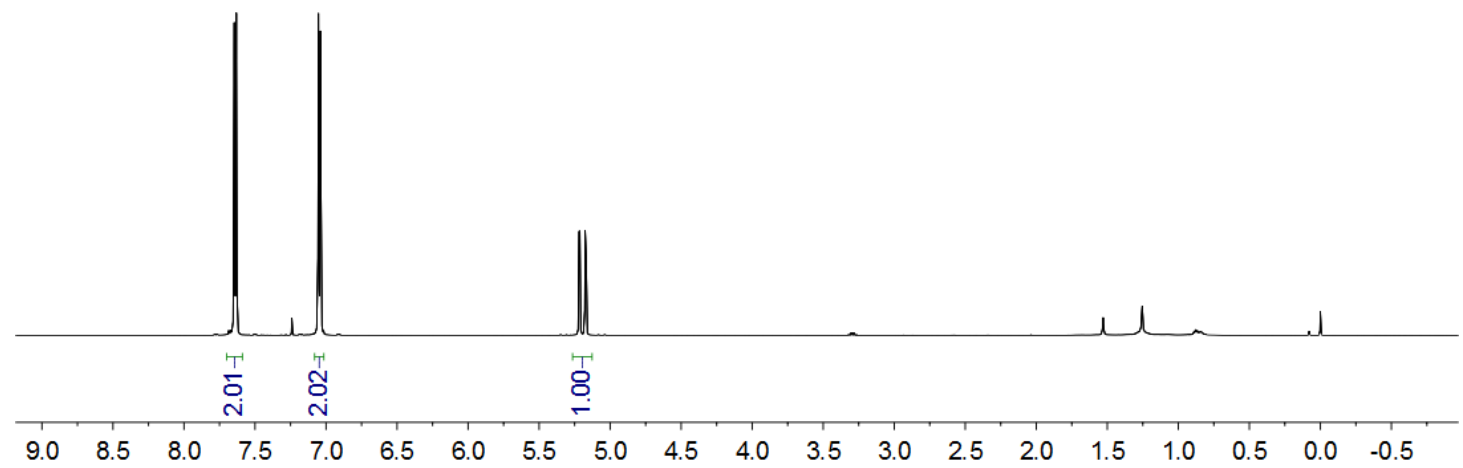



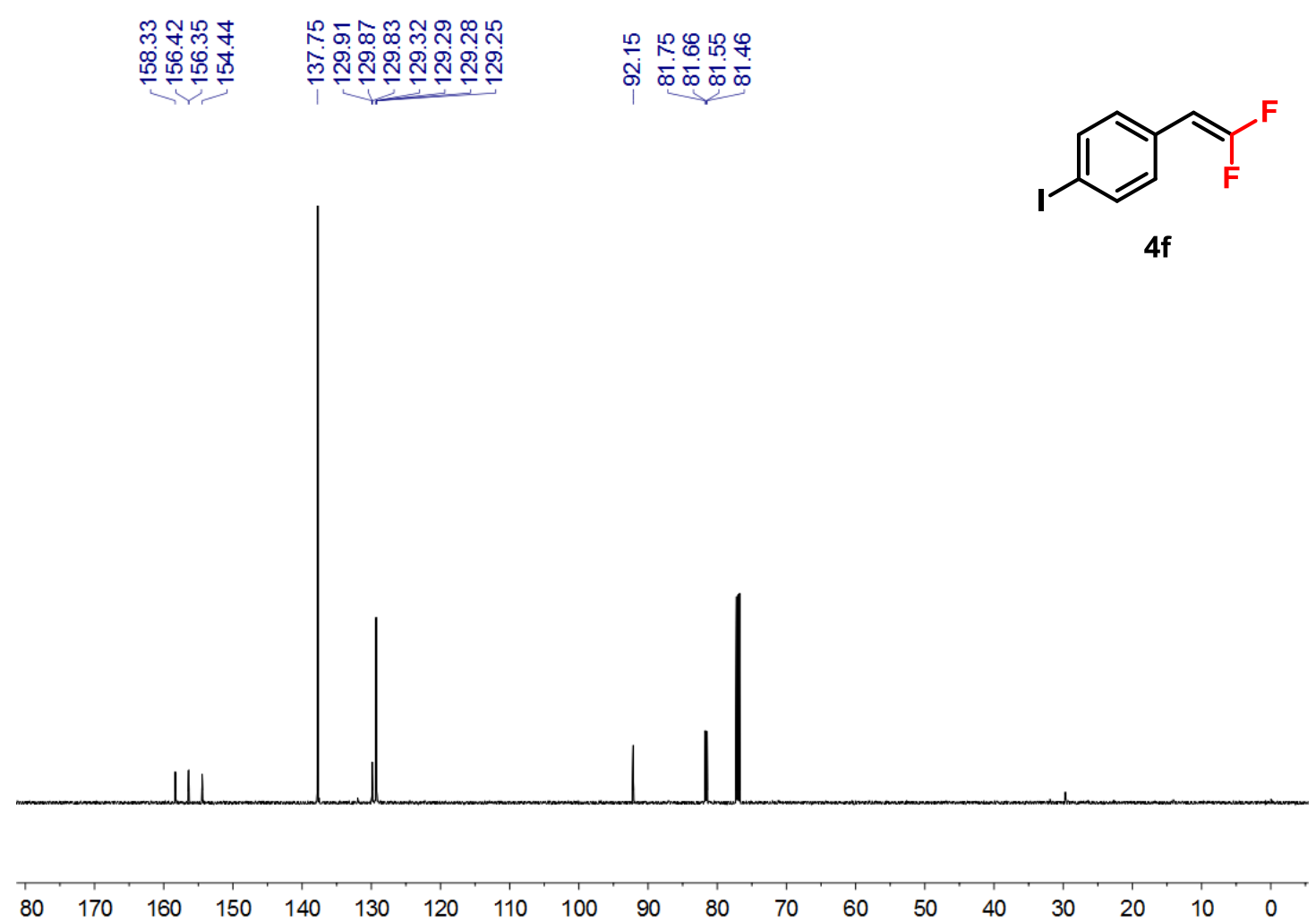

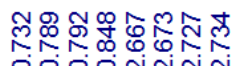

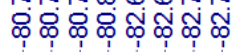
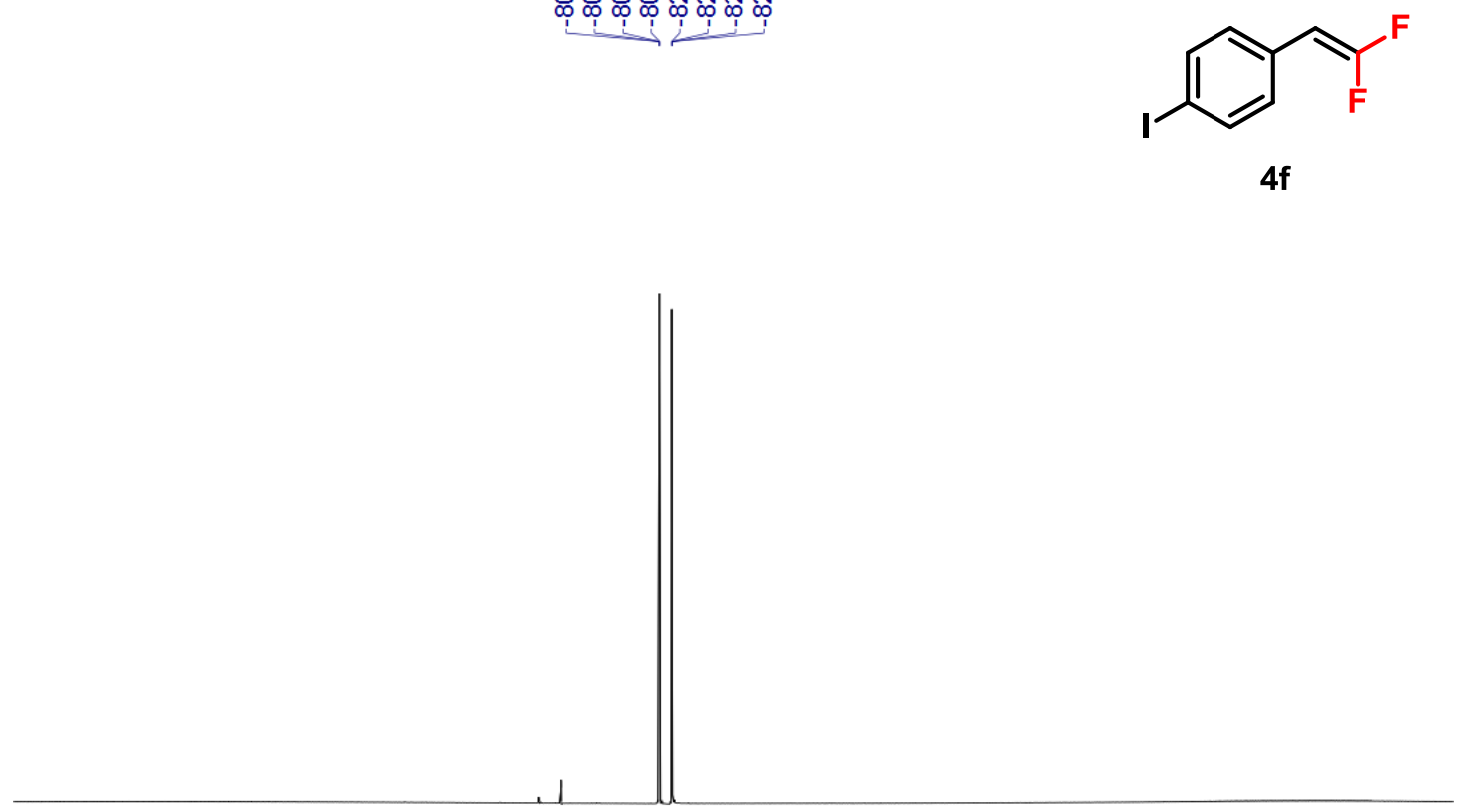

$\begin{array}{lllllllllllllllll}10 & 0 & -10 & -20 & -30 & -40 & -50 & -60 & -70 & -80 & -90 & -110 & -130 & -150 & -170 & -190\end{array}$ 


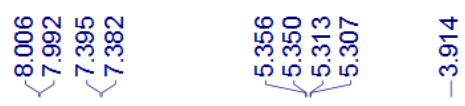

${ }_{\mathrm{MeO}_{2}} \mathrm{C}_{\mathrm{F}}^{\mathrm{F}}$

$4 \mathrm{~g}$

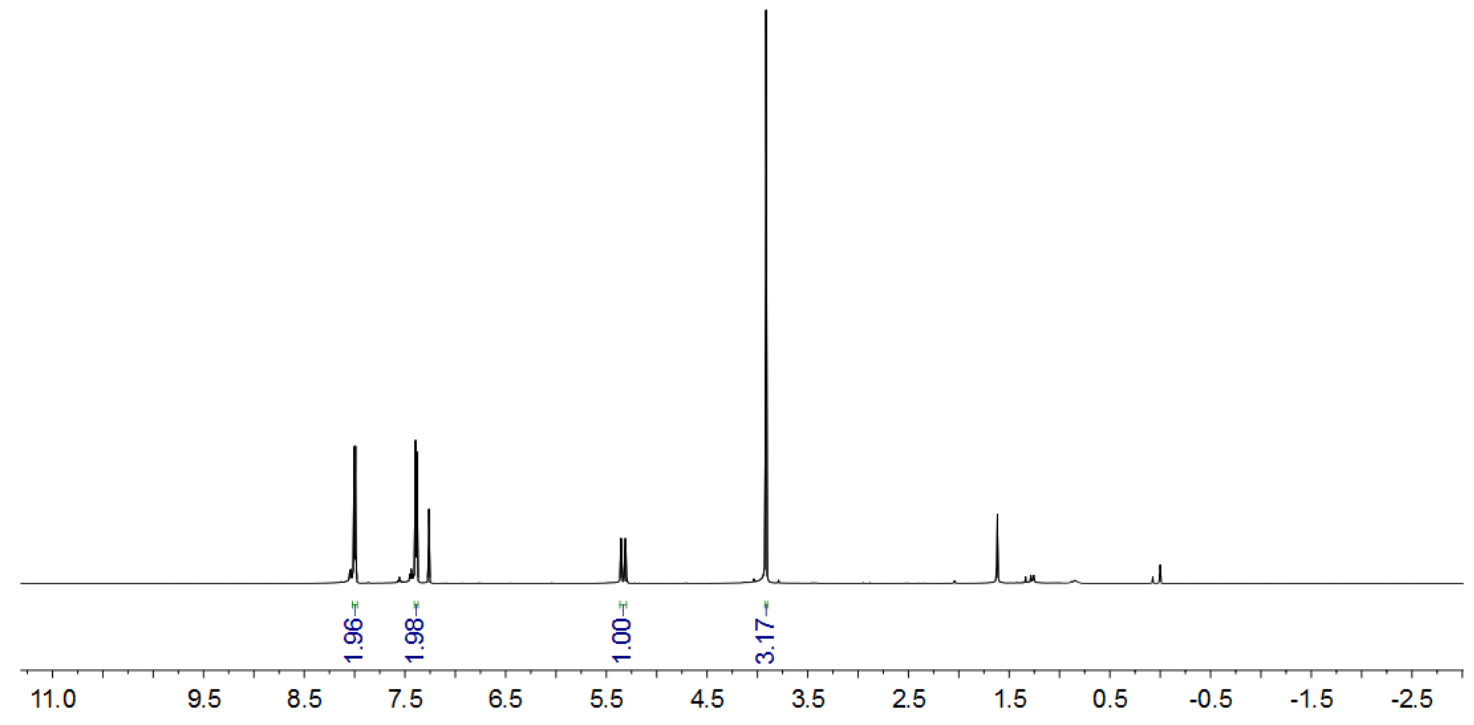

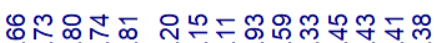
ڤั

$\underbrace{050 \%}$<smiles>COC(=O)c1ccc(C=C(F)F)cc1</smiles>

$4 \mathrm{~g}$

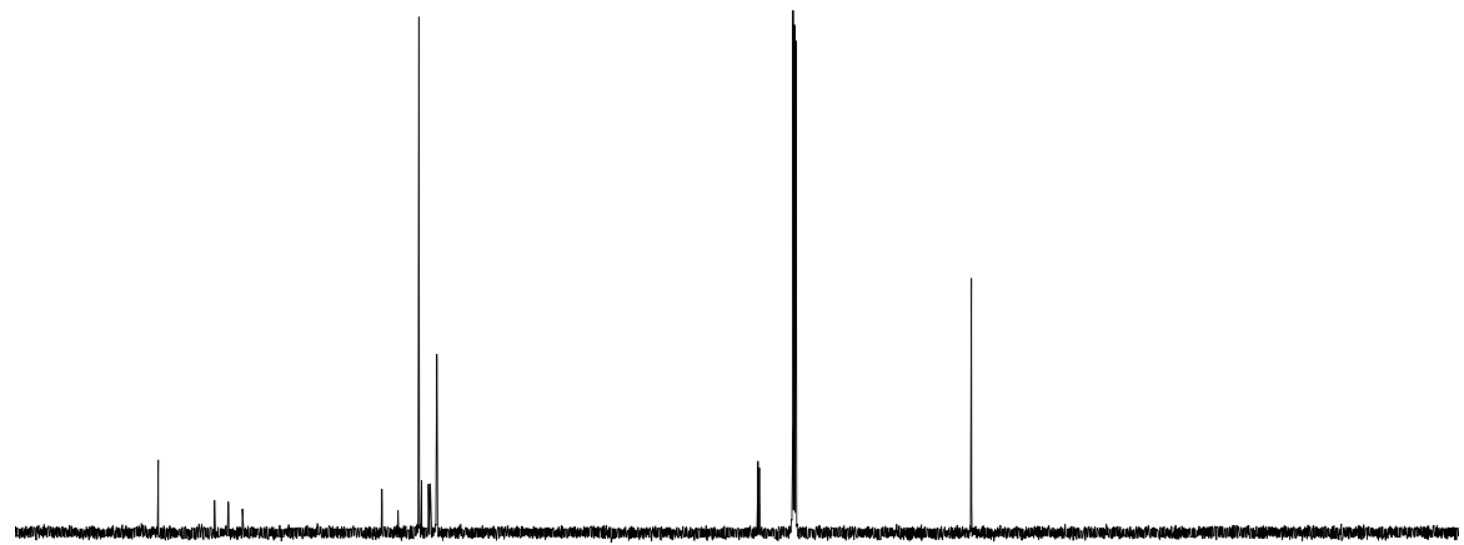

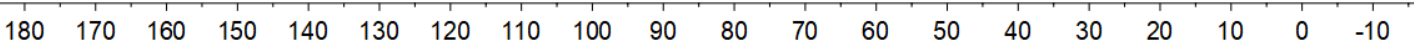




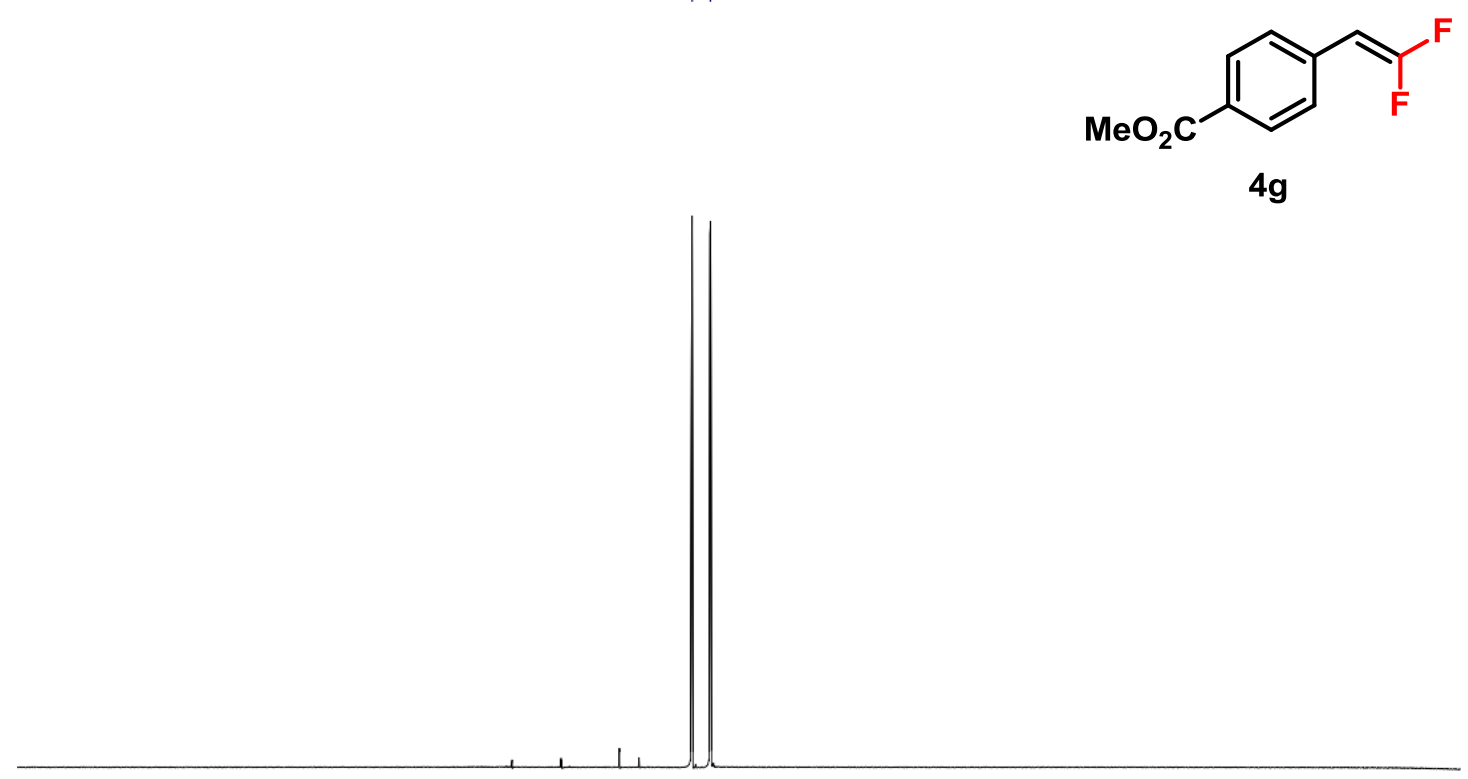

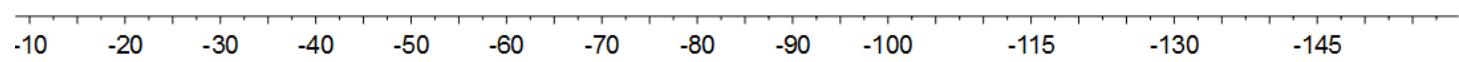

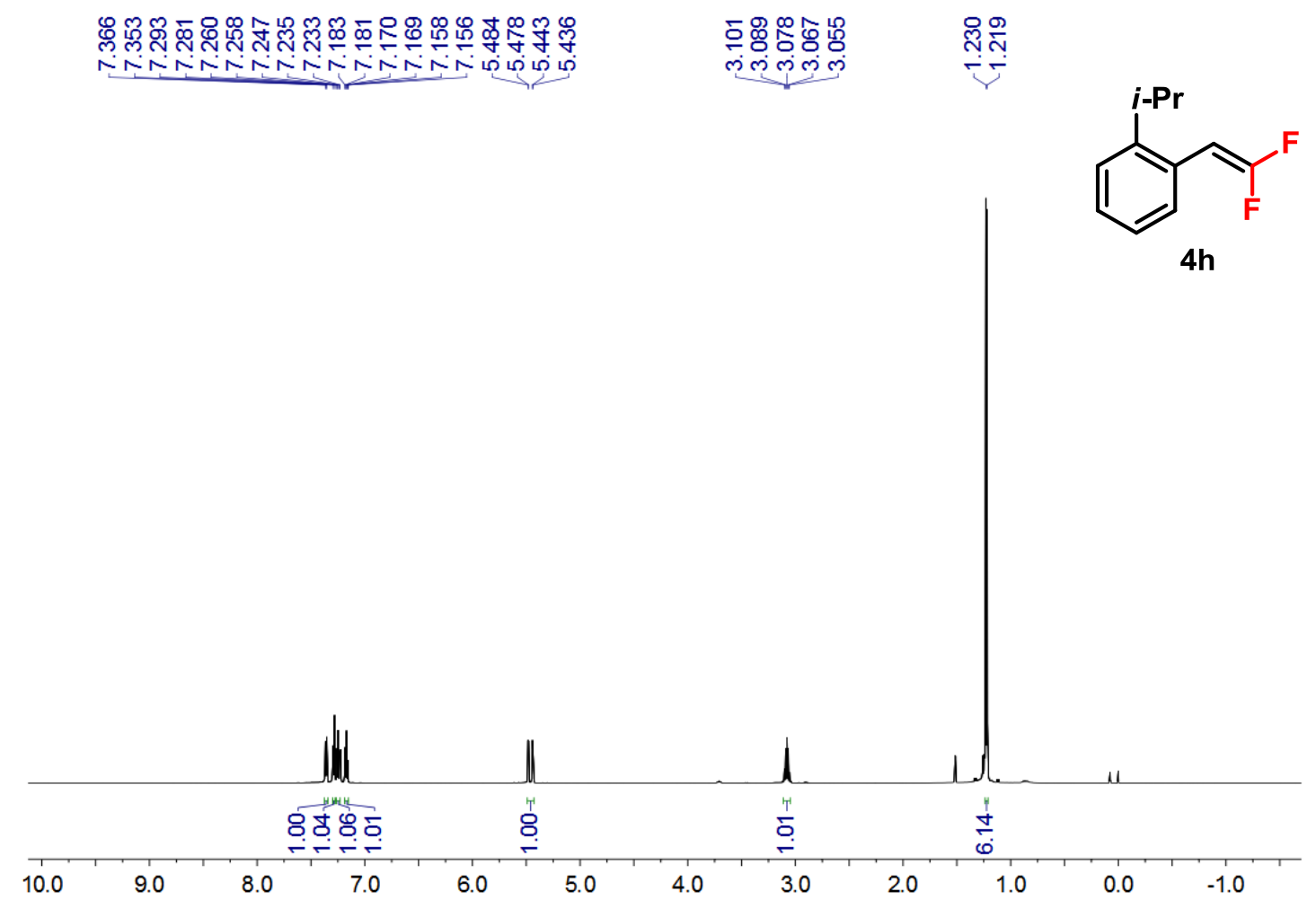




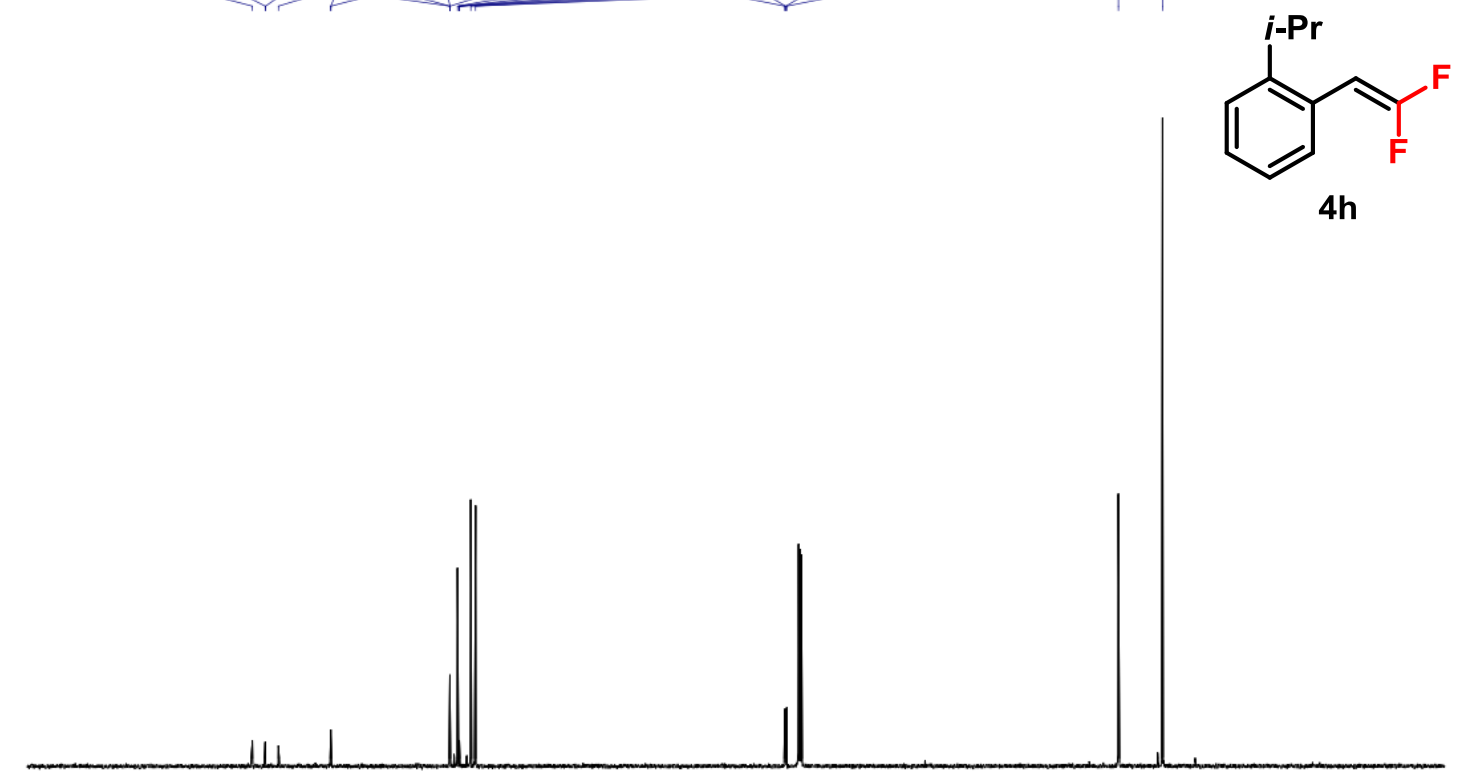

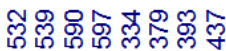

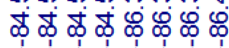
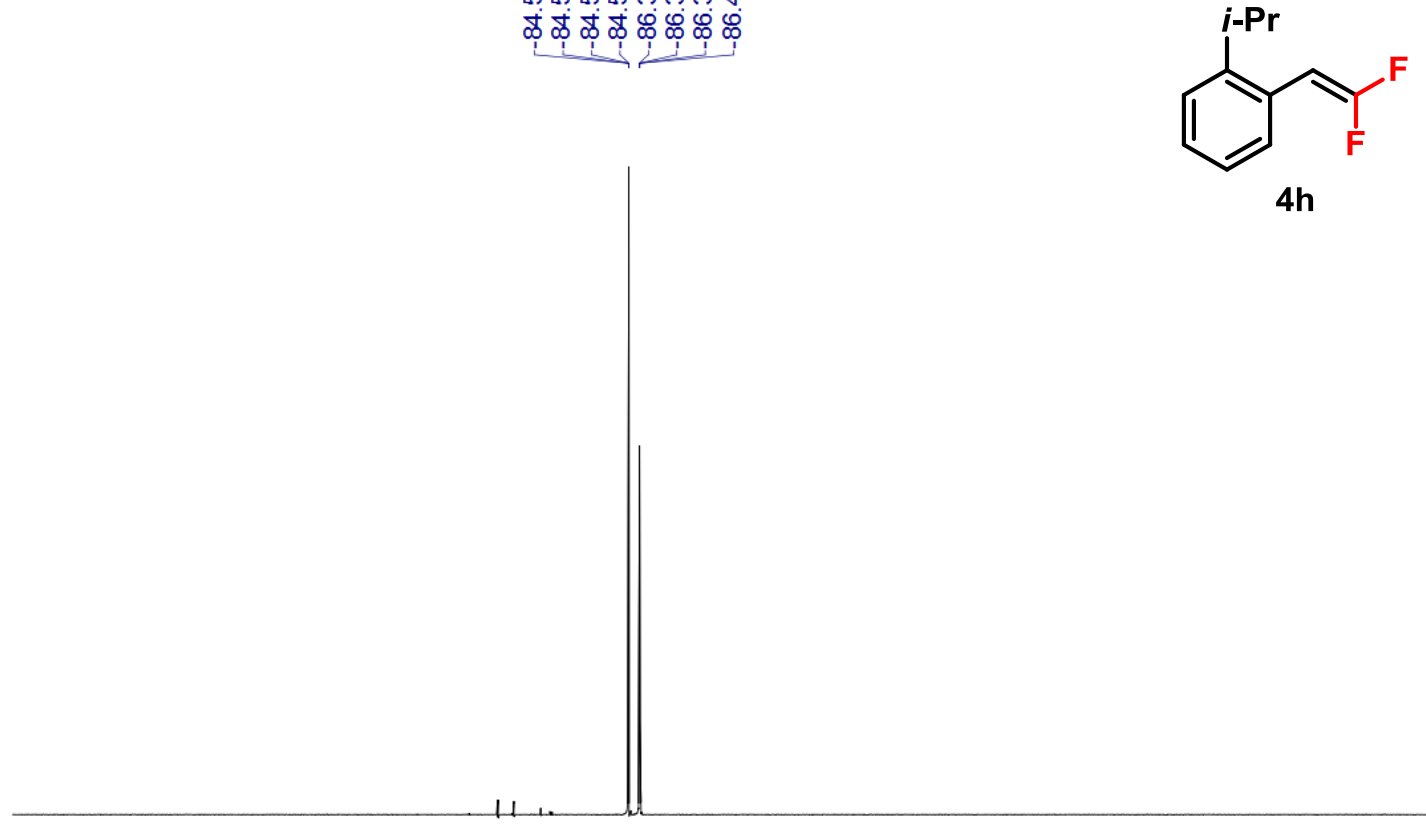

100

$-20$

$-40$

$-80$

$-100$

$-160$

$-180$

$-200$ 


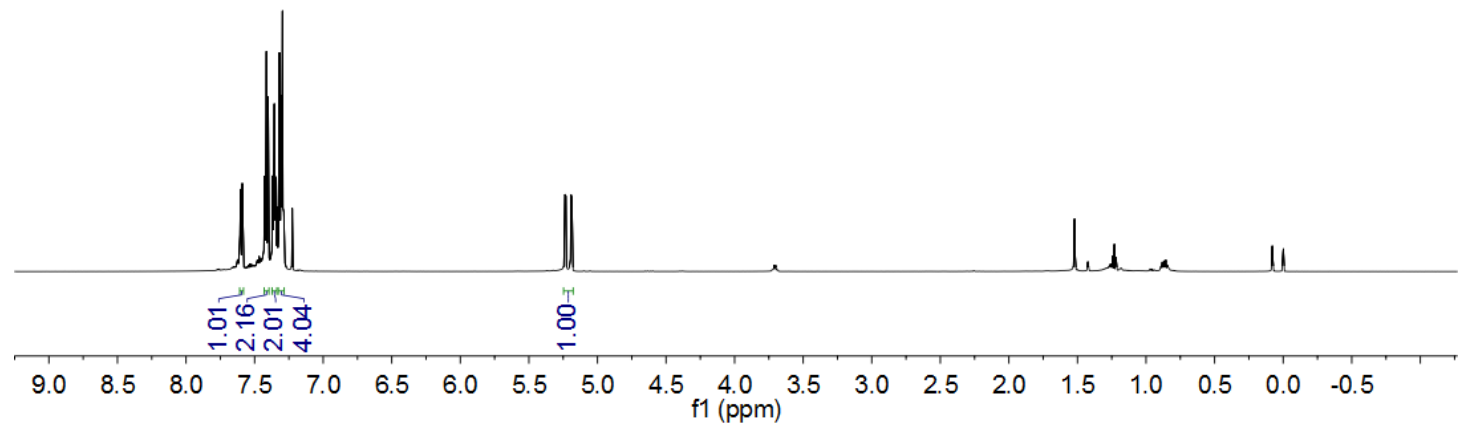

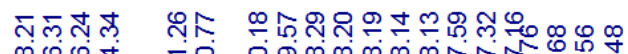

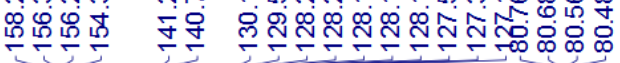

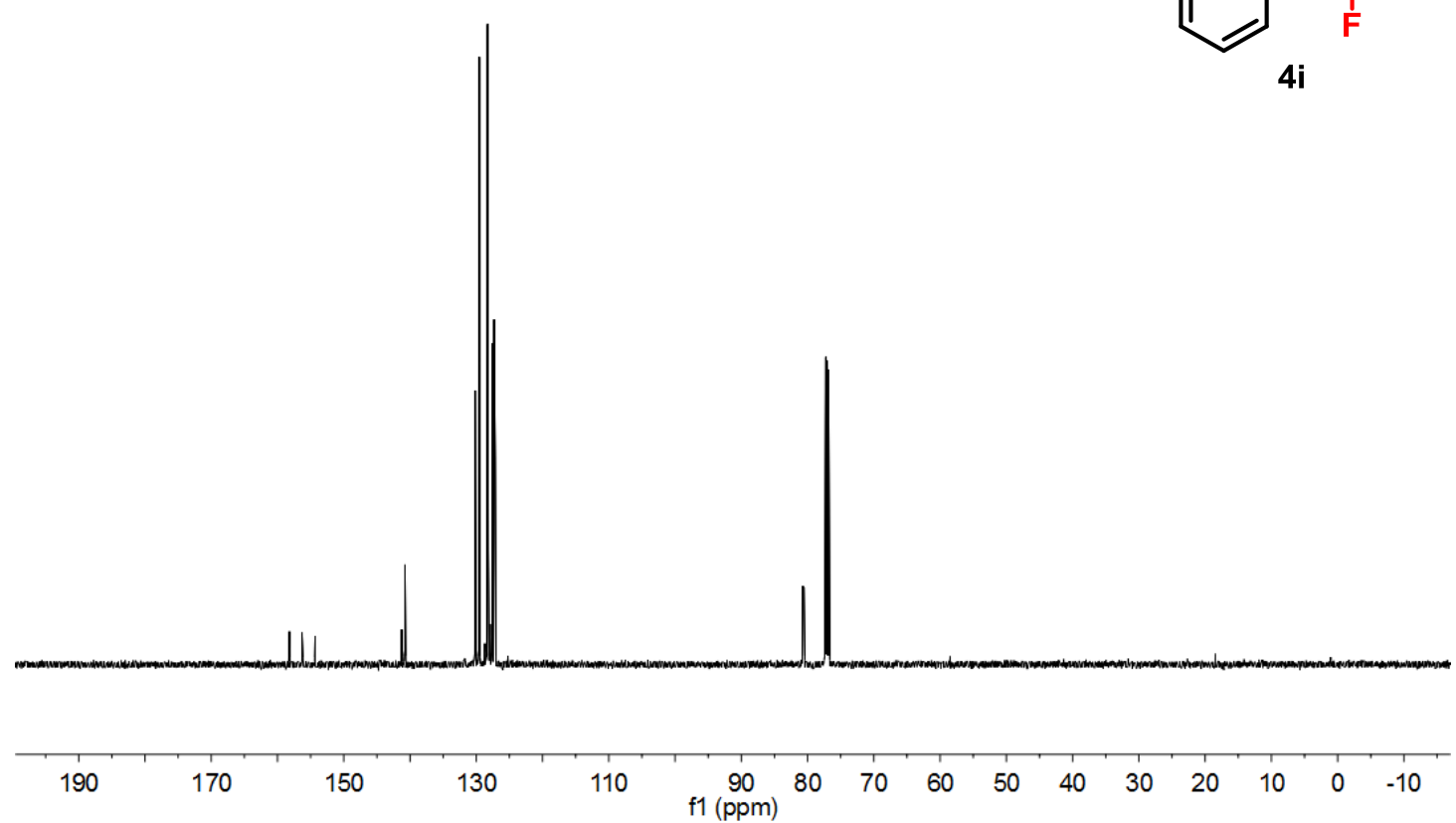<smiles>FC(F)=Cc1ccccc1-c1ccccc1</smiles> 


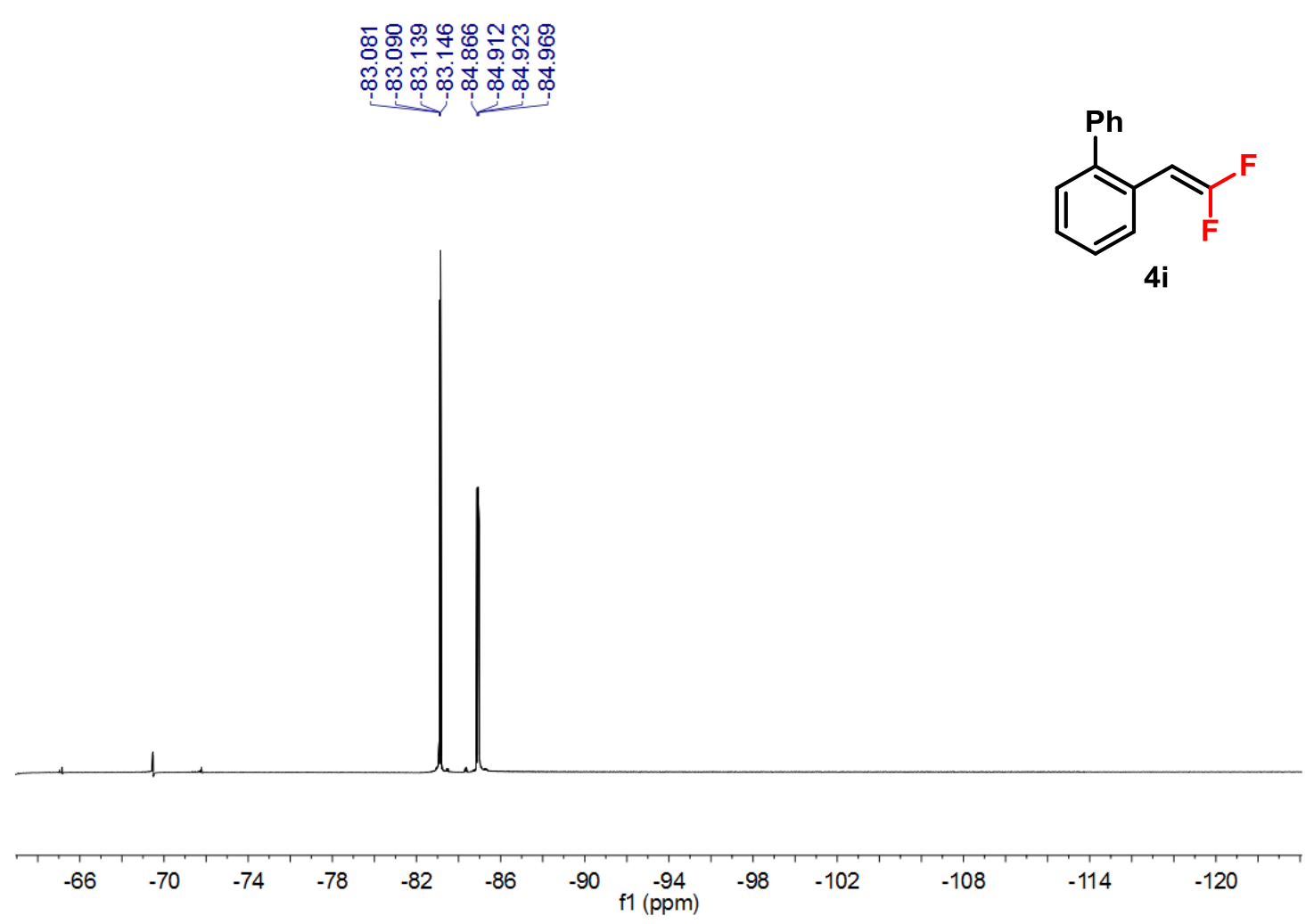

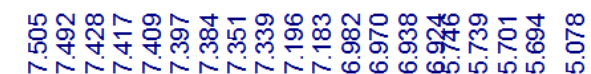

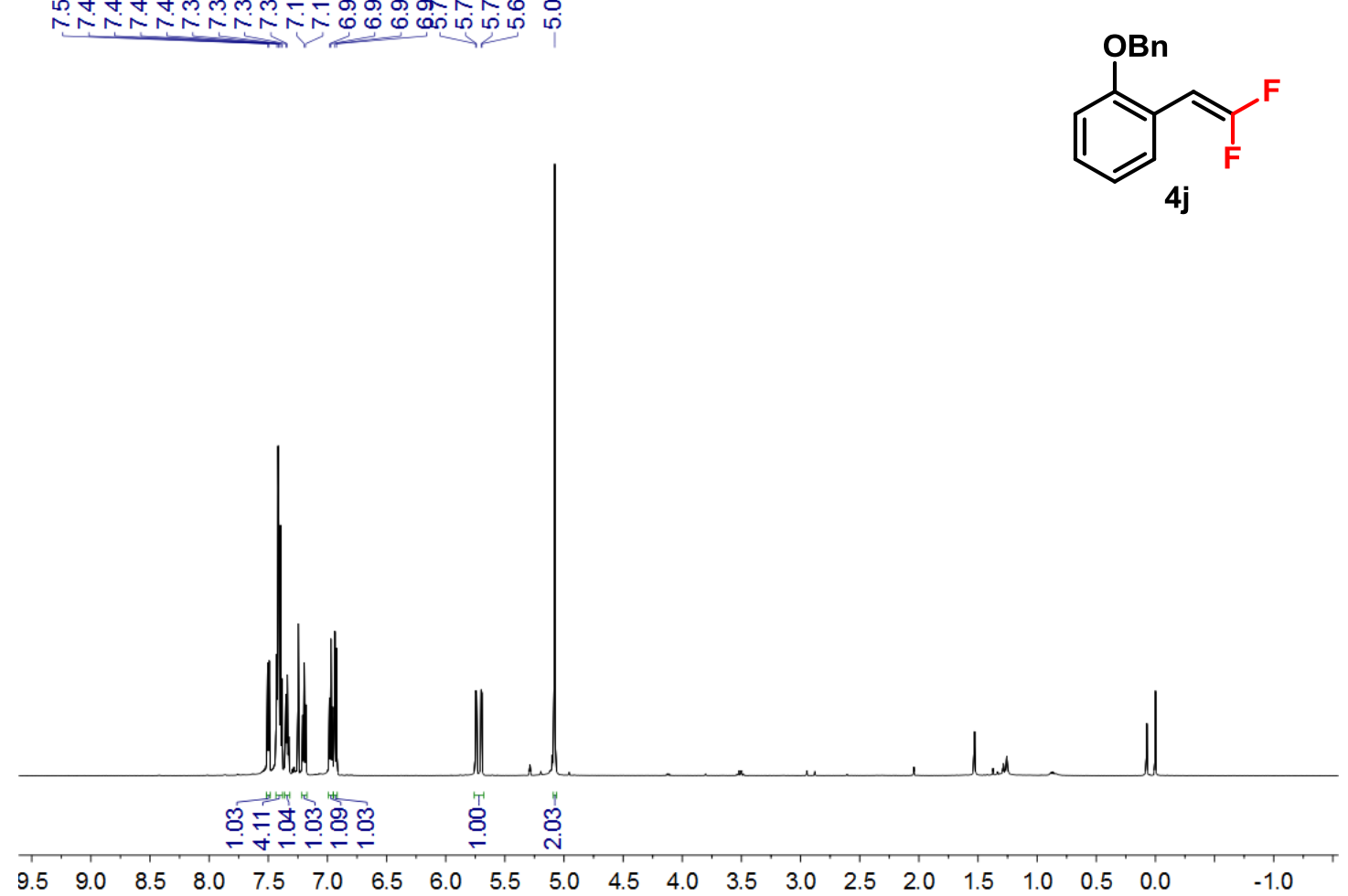




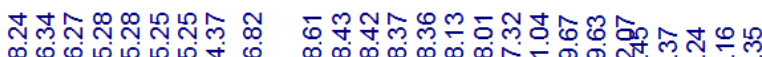

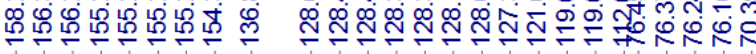

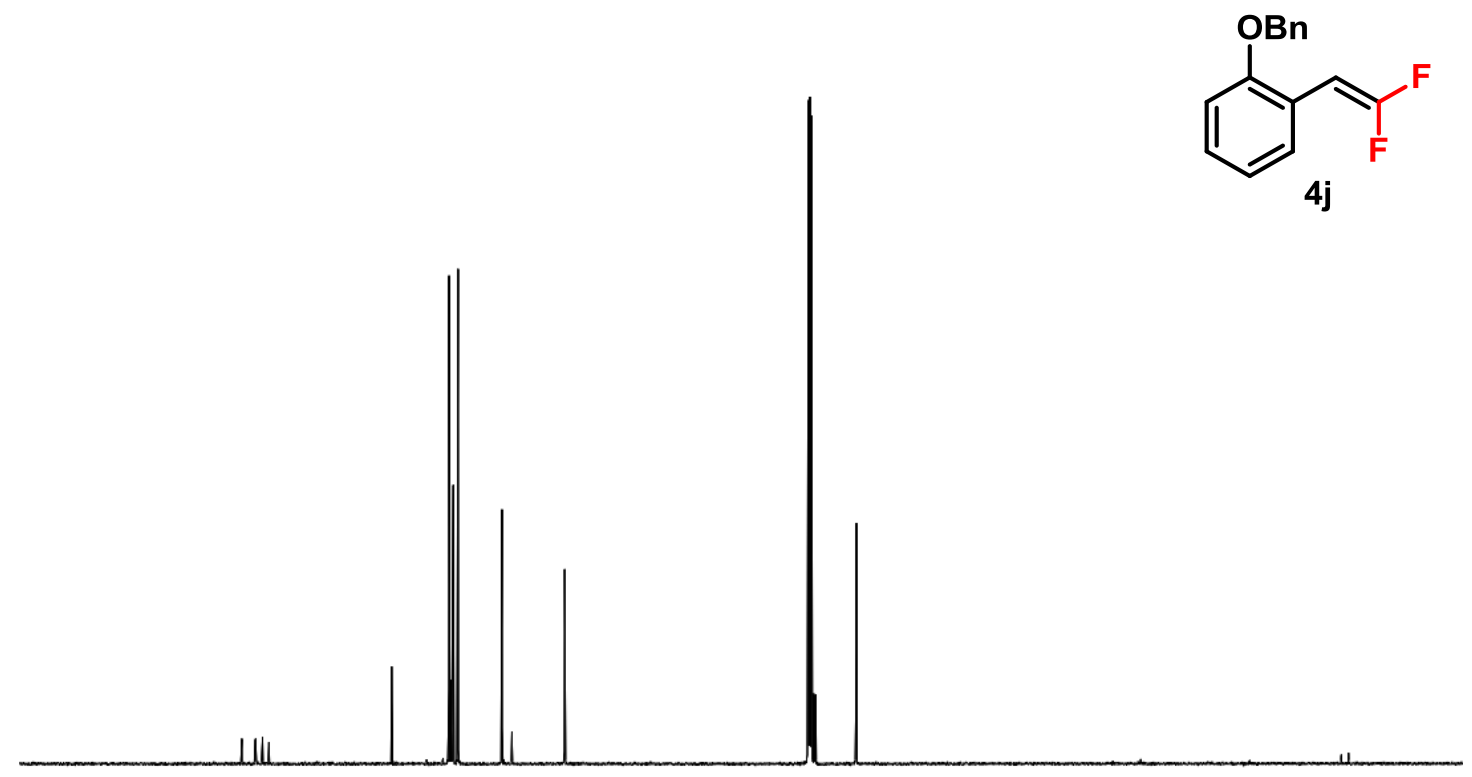

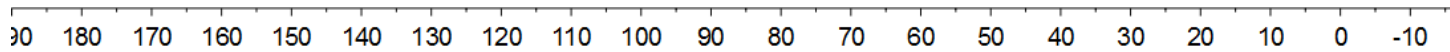

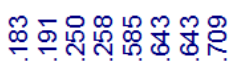

๗ळ
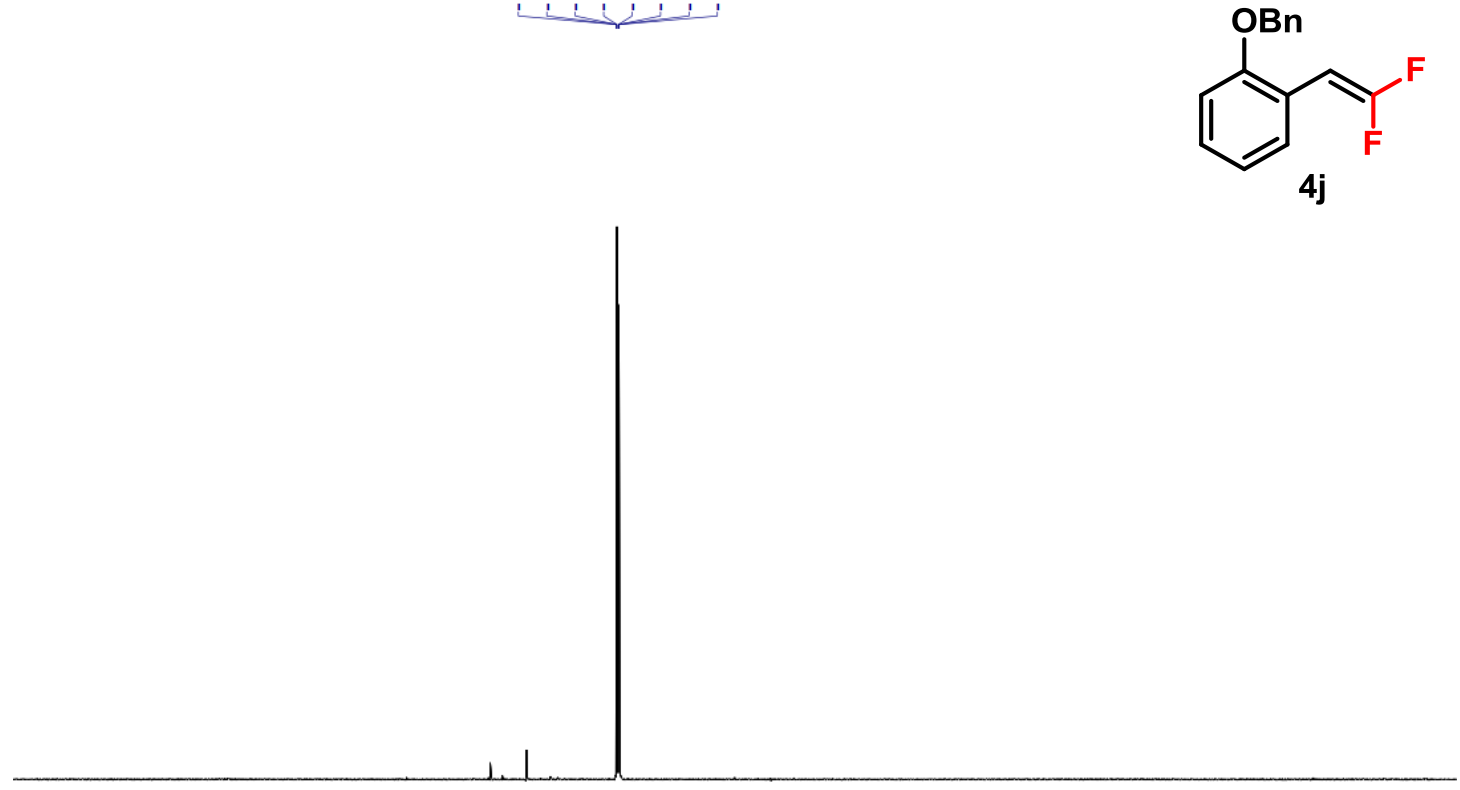

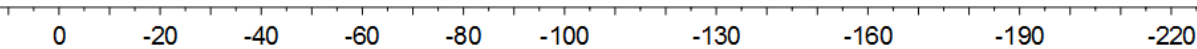



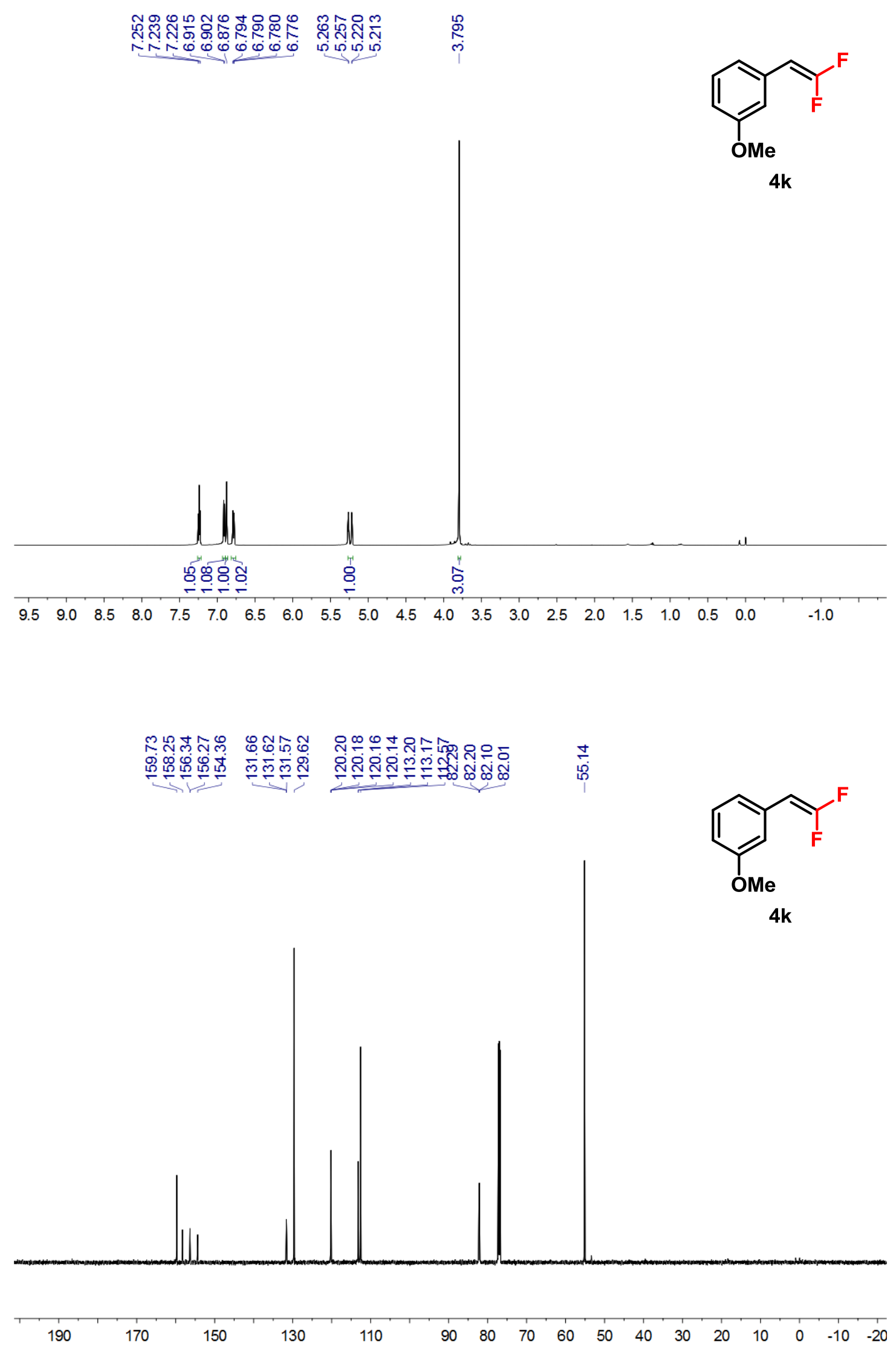


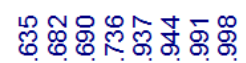

कळ ळळळ ळ
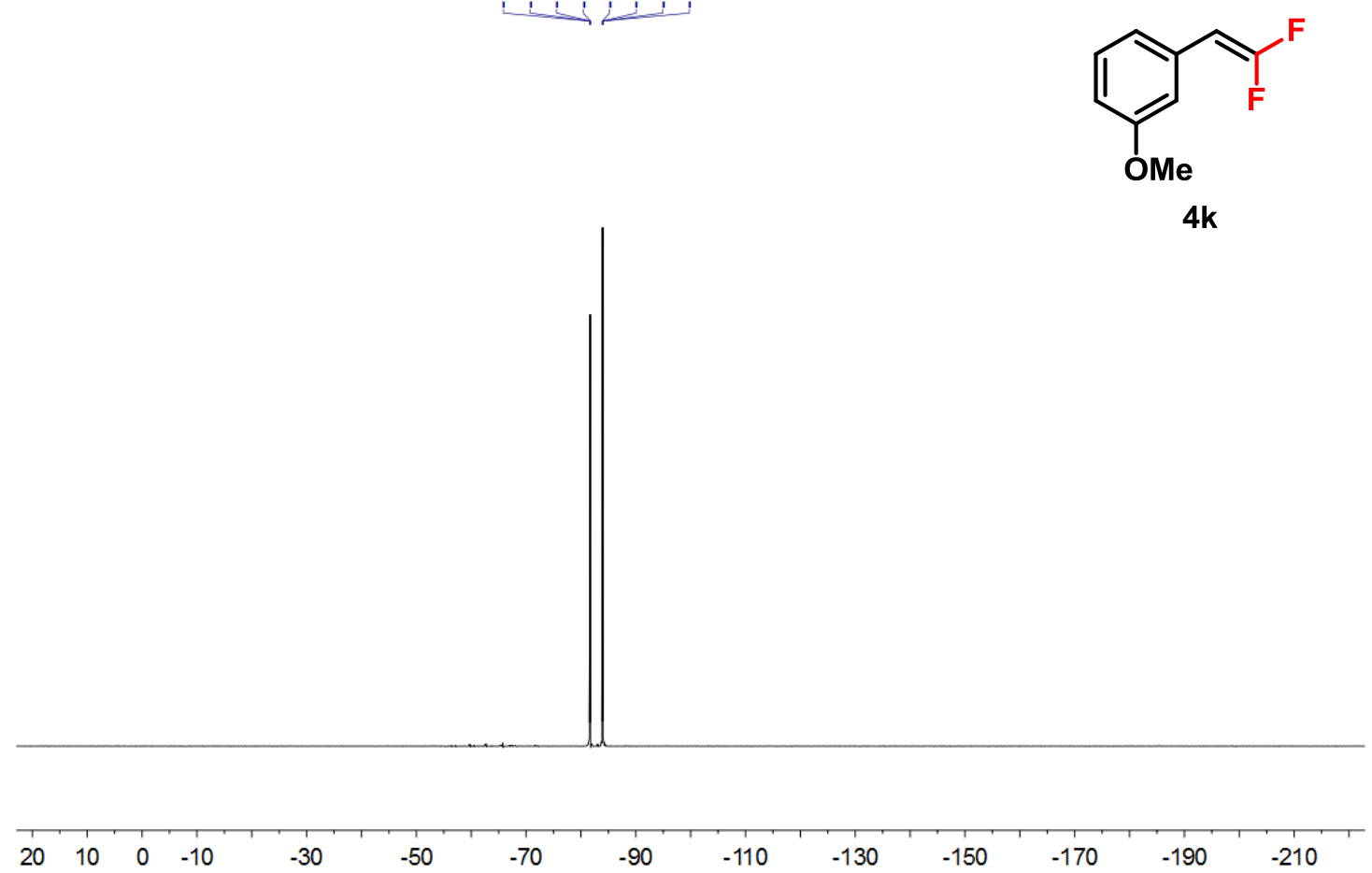

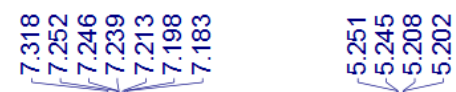

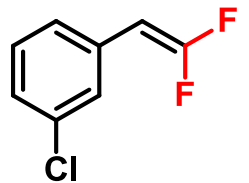

41

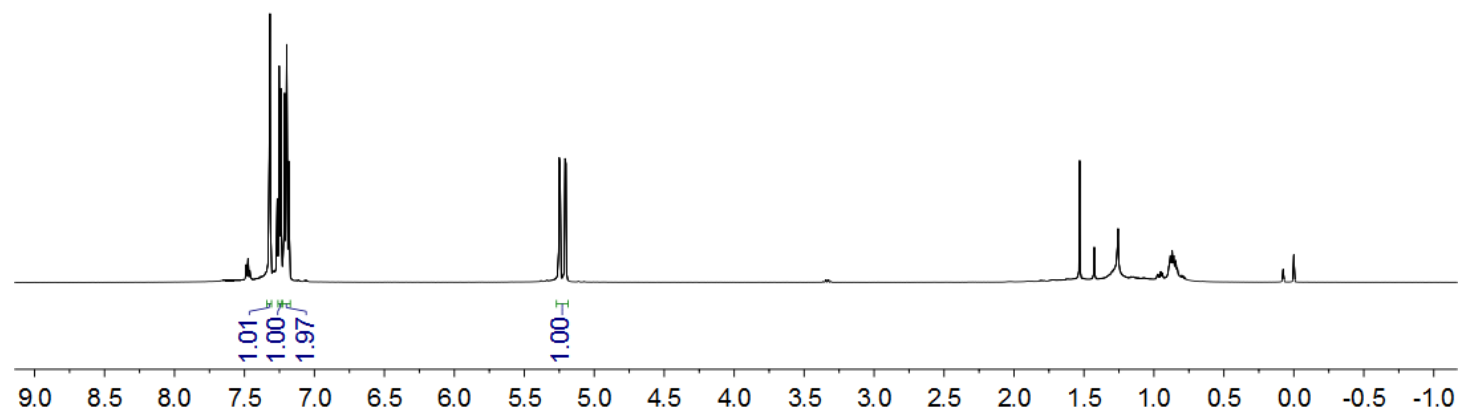




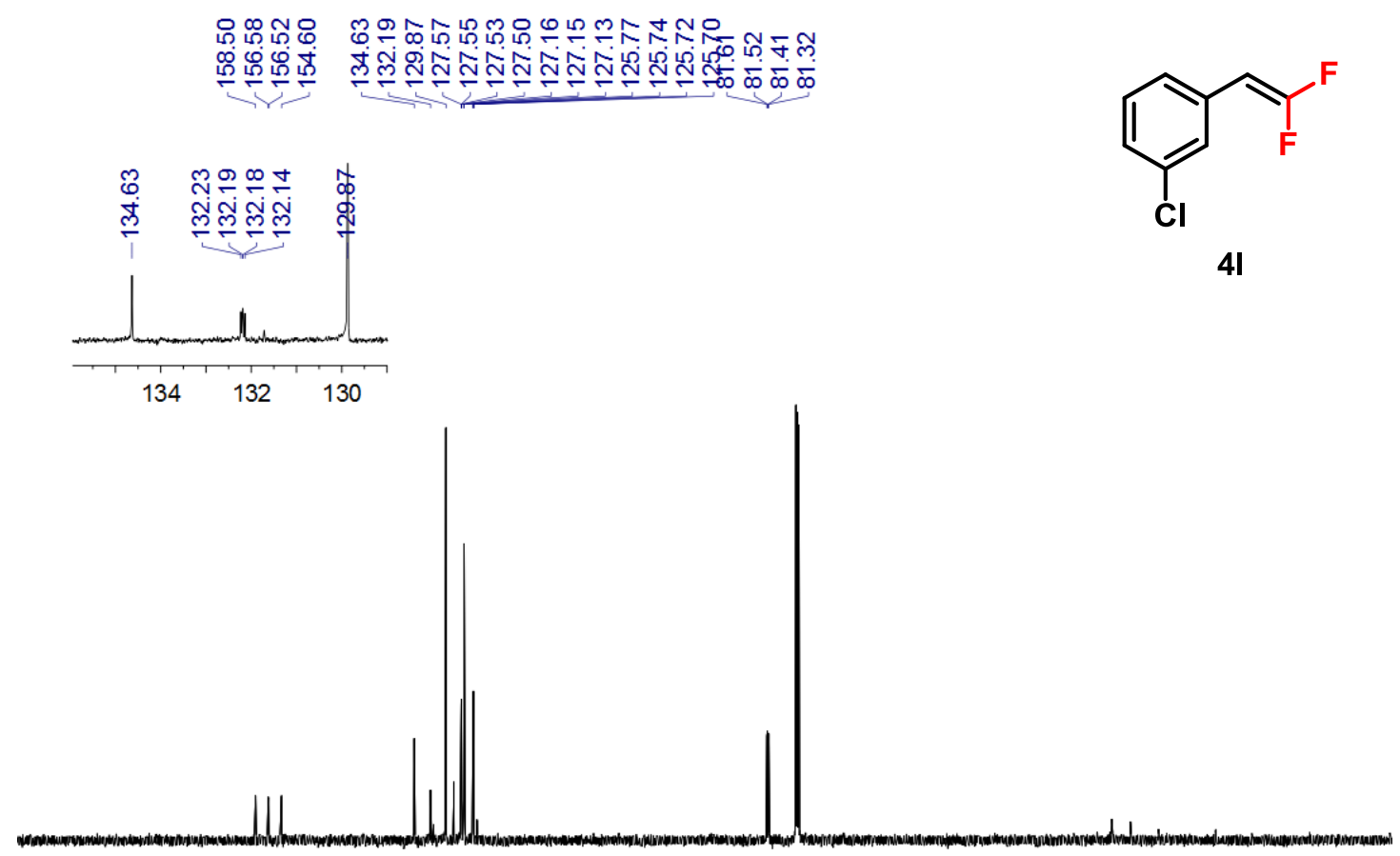

$\begin{array}{lllllllllllllllllllll}190 & 180 & 170 & 160 & 150 & 140 & 130 & 120 & 110 & 100 & 90 & 80 & 70 & 60 & 50 & 40 & 30 & 20 & 10 & 0 & -10\end{array}$

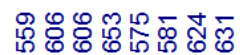

ळి ळ

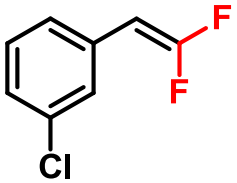

4I

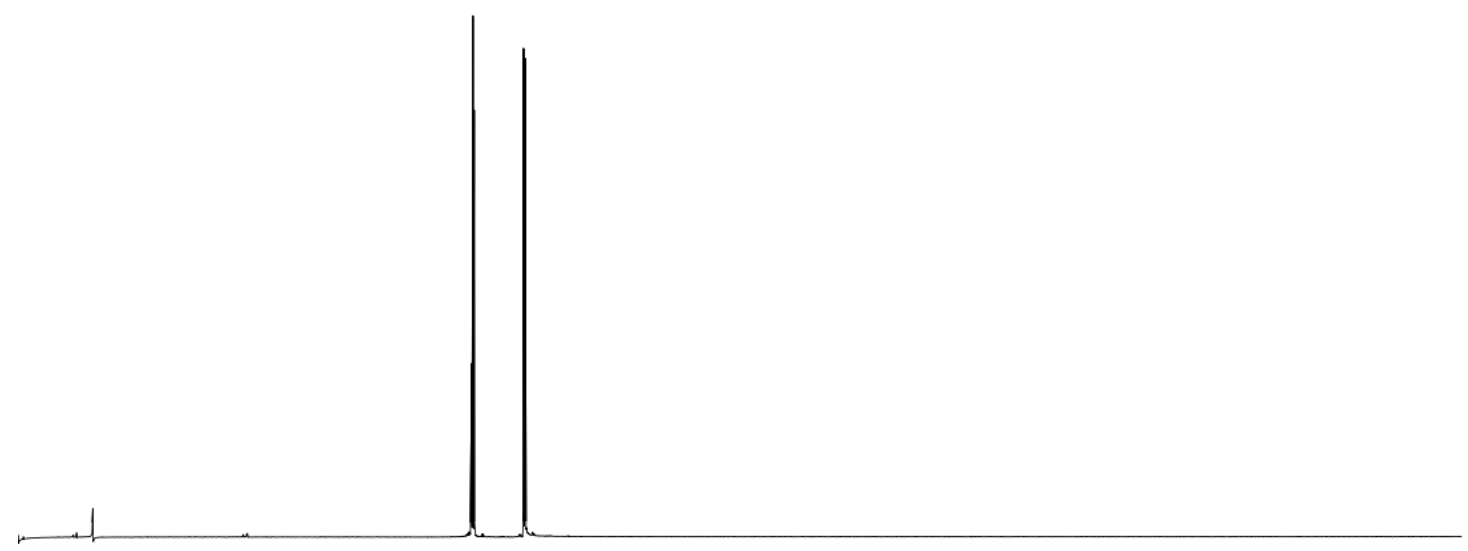

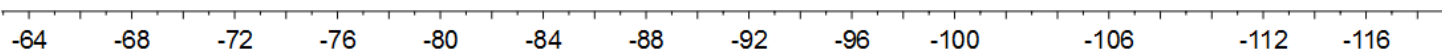



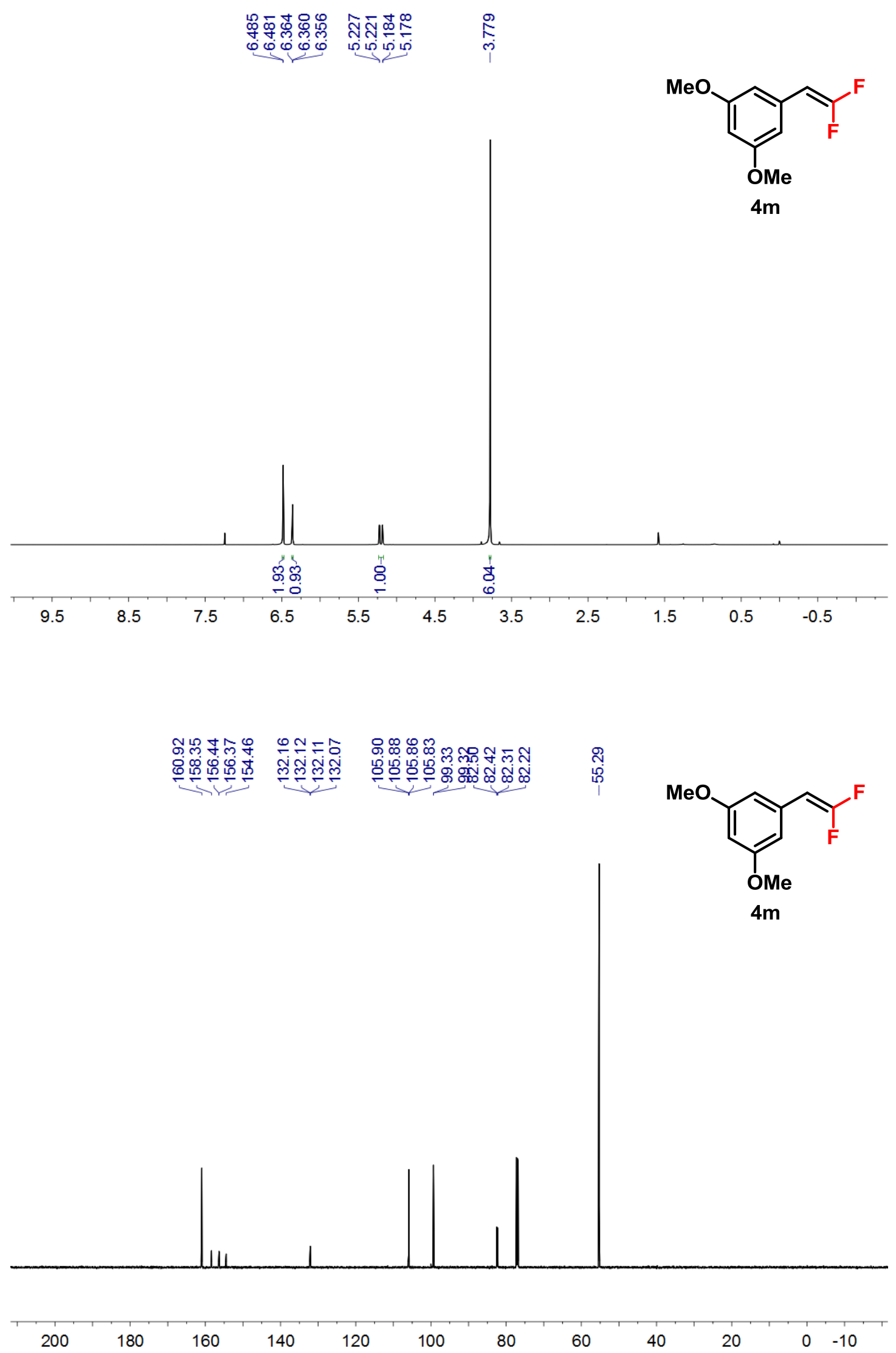

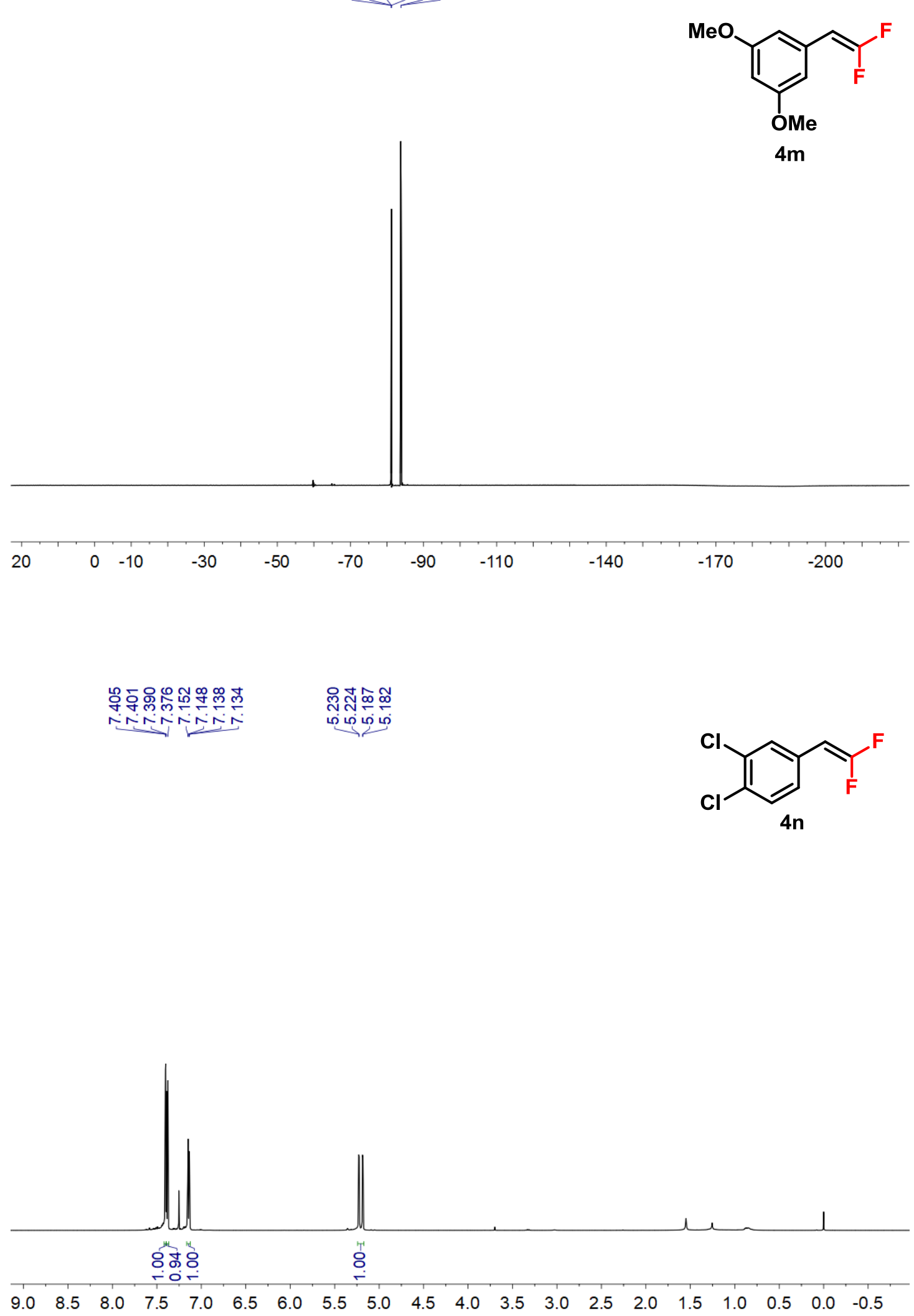
<smiles>FC(F)=Cc1cc(Cl)c(Cl)c(Cl)c1</smiles>

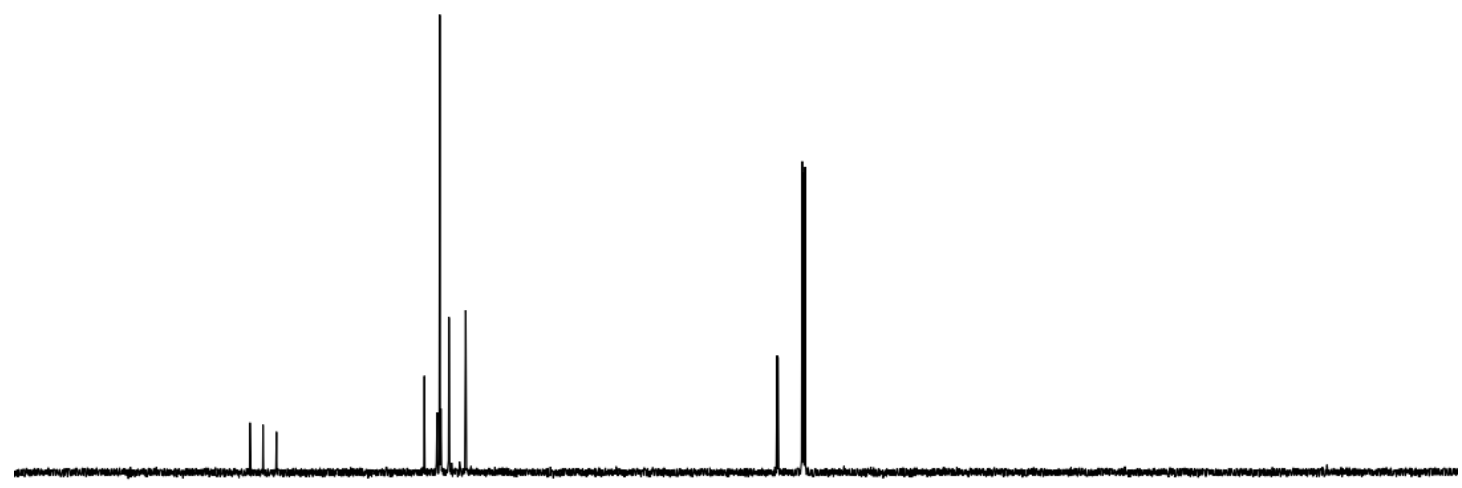

$\begin{array}{lllllllllllllllllllll}190 & 180 & 170 & 160 & 150 & 140 & 130 & 120 & 110 & 100 & 90 & 80 & 70 & 60 & 50 & 40 & 30 & 20 & 10 & 0 & -10\end{array}$

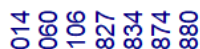

क्षक्ष<smiles>FC(F)=Cc1cc(Cl)c(Cl)c(Cl)c1</smiles>

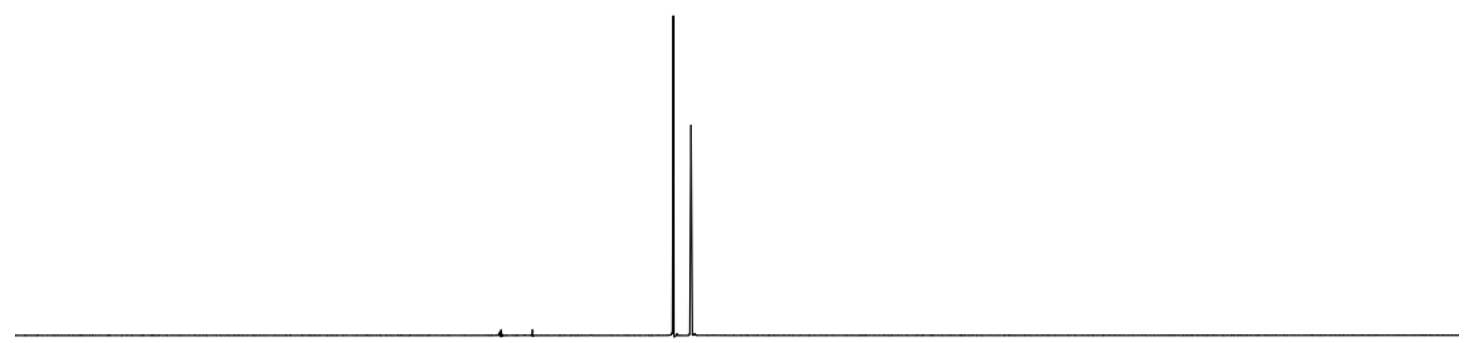




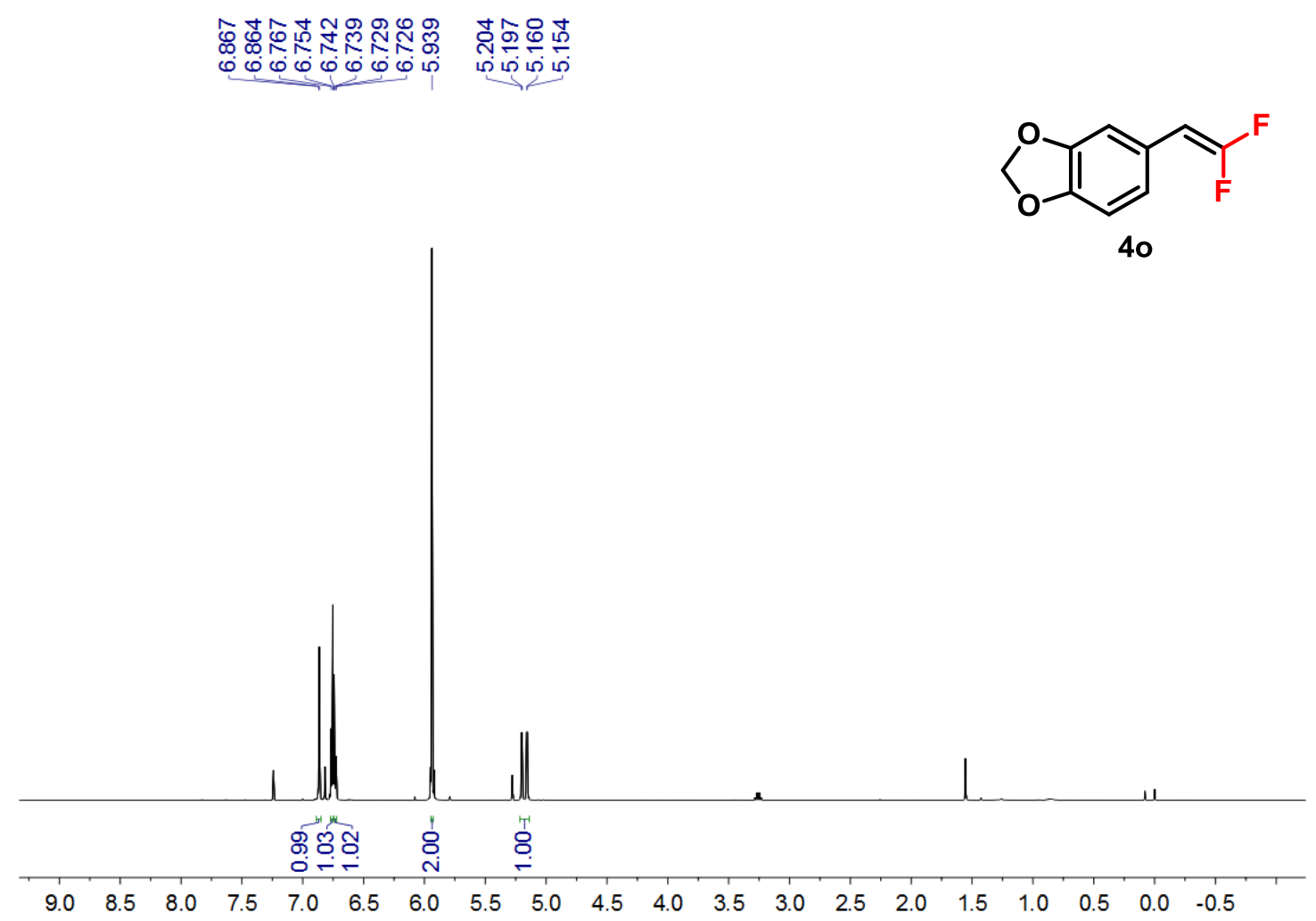

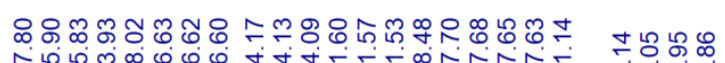

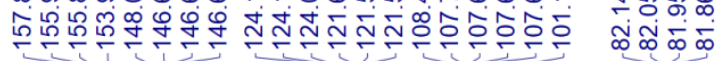

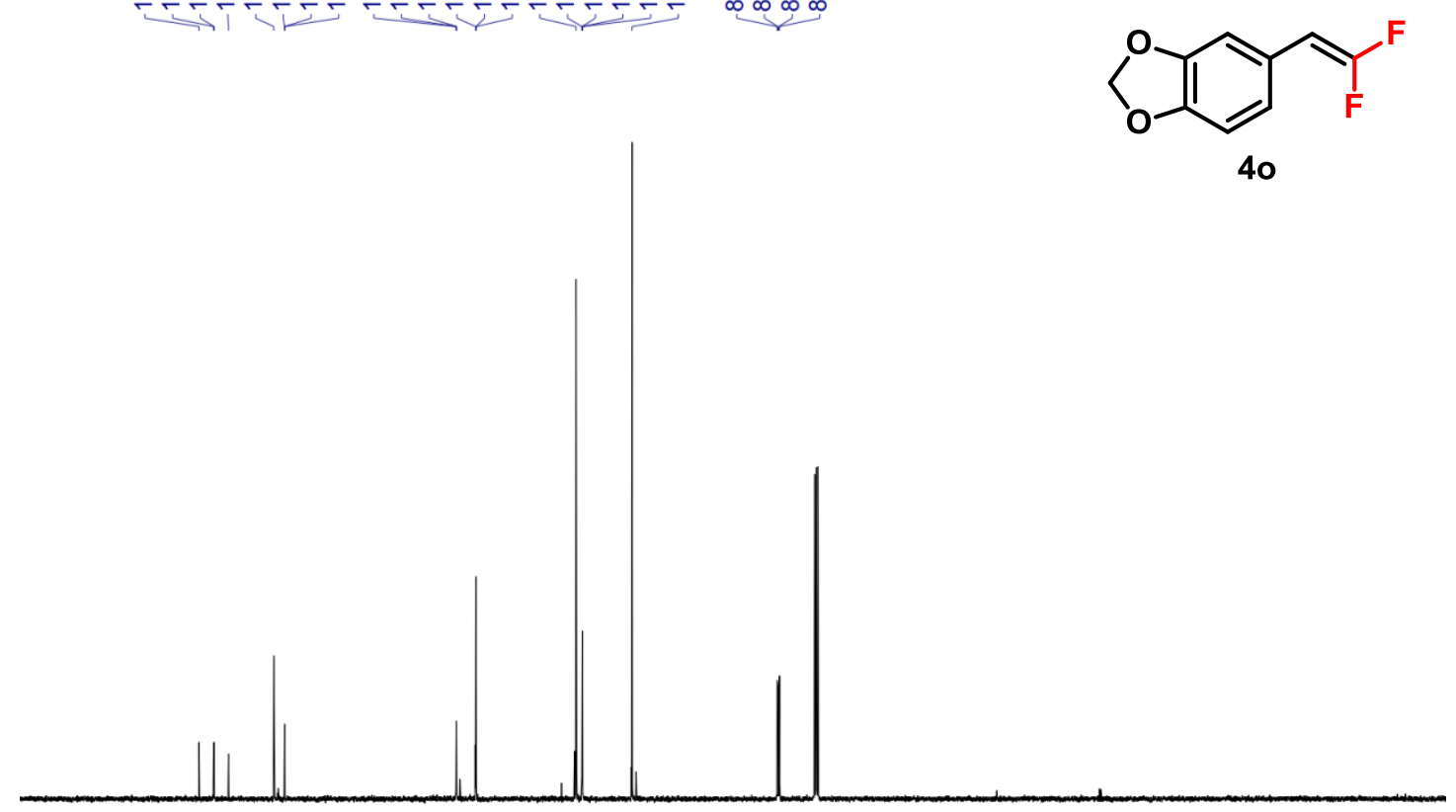

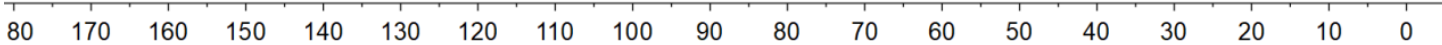




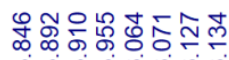

ळळळळळळळ
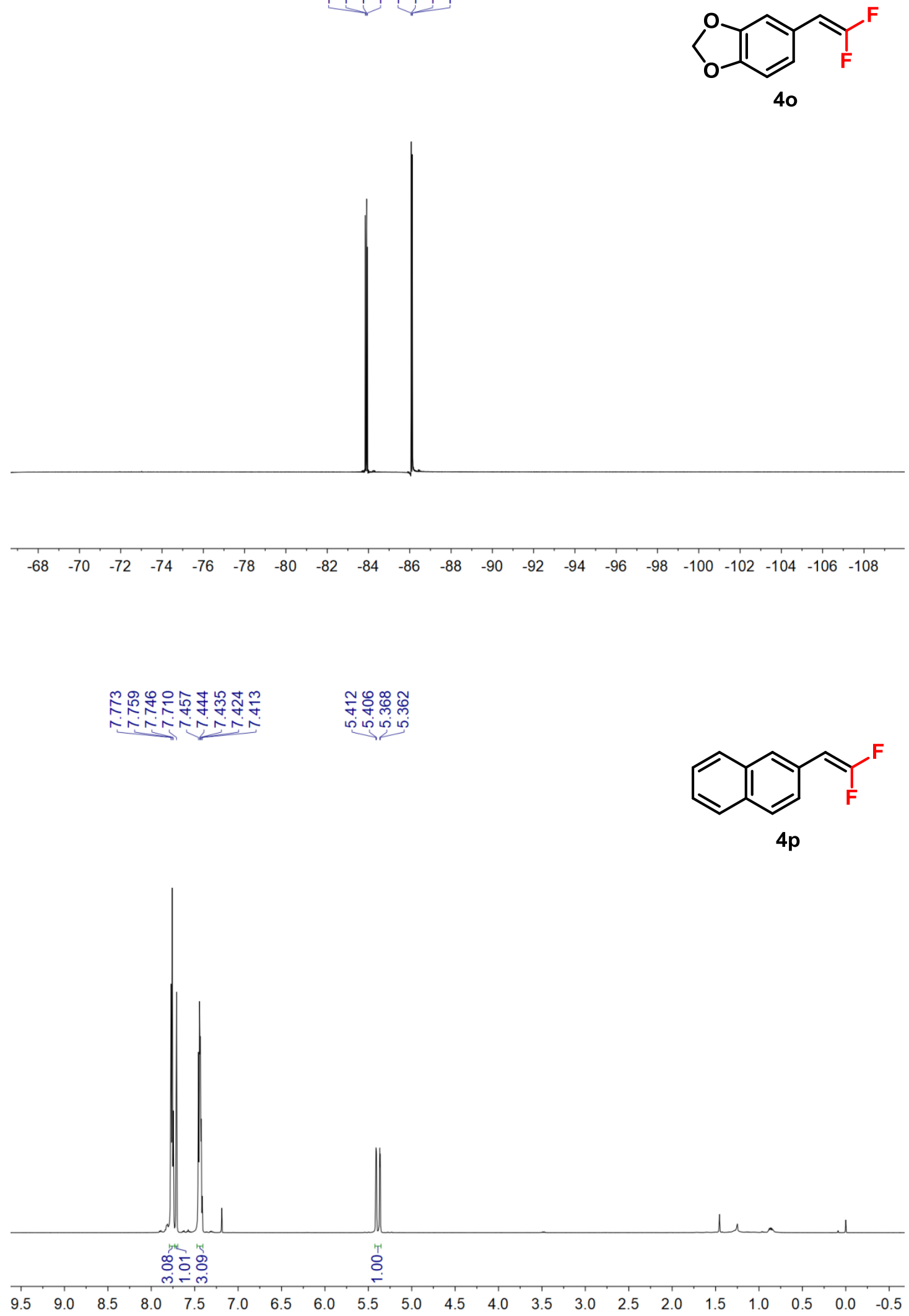

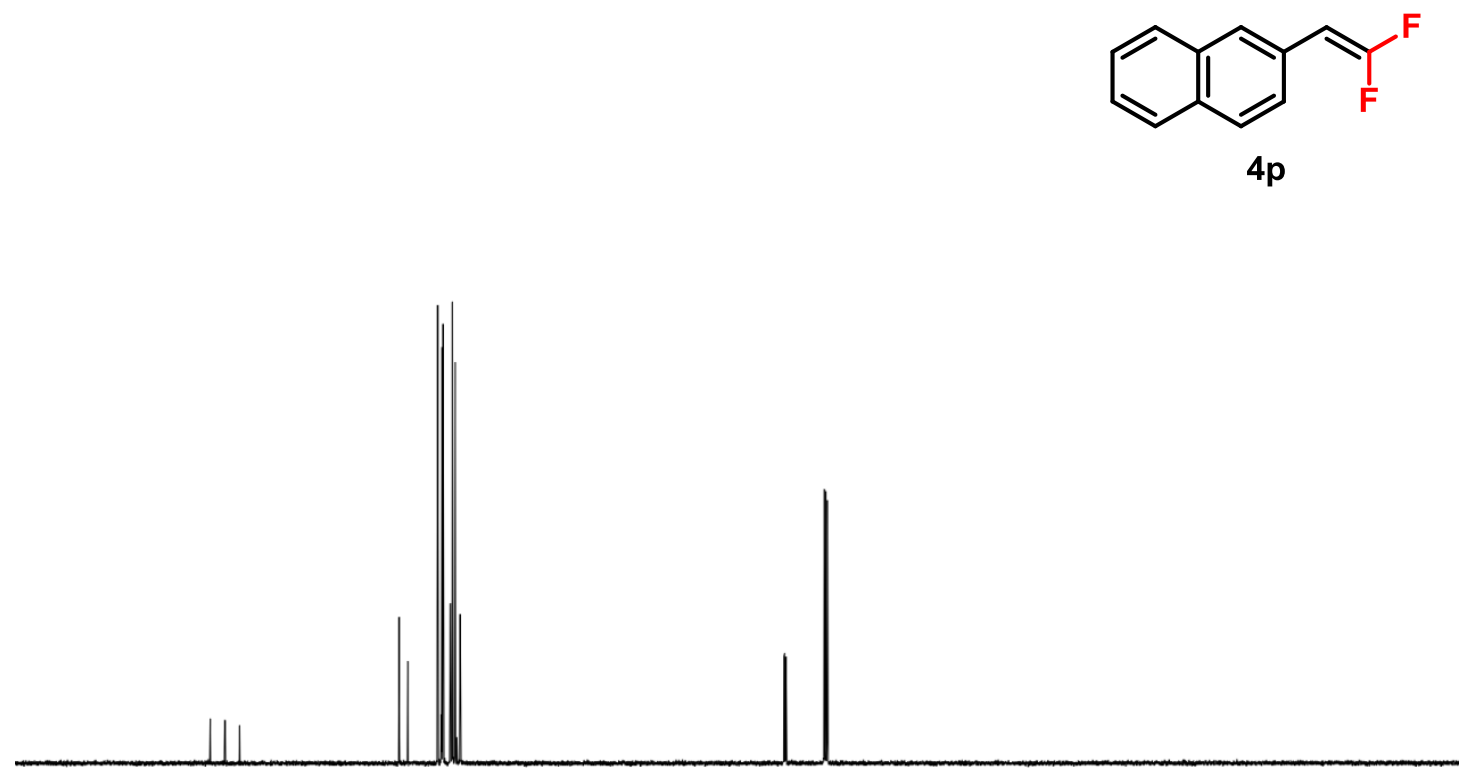

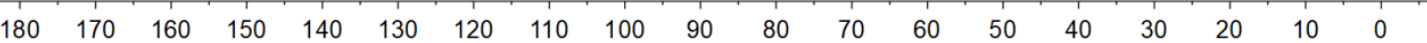

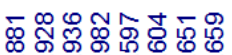

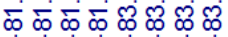
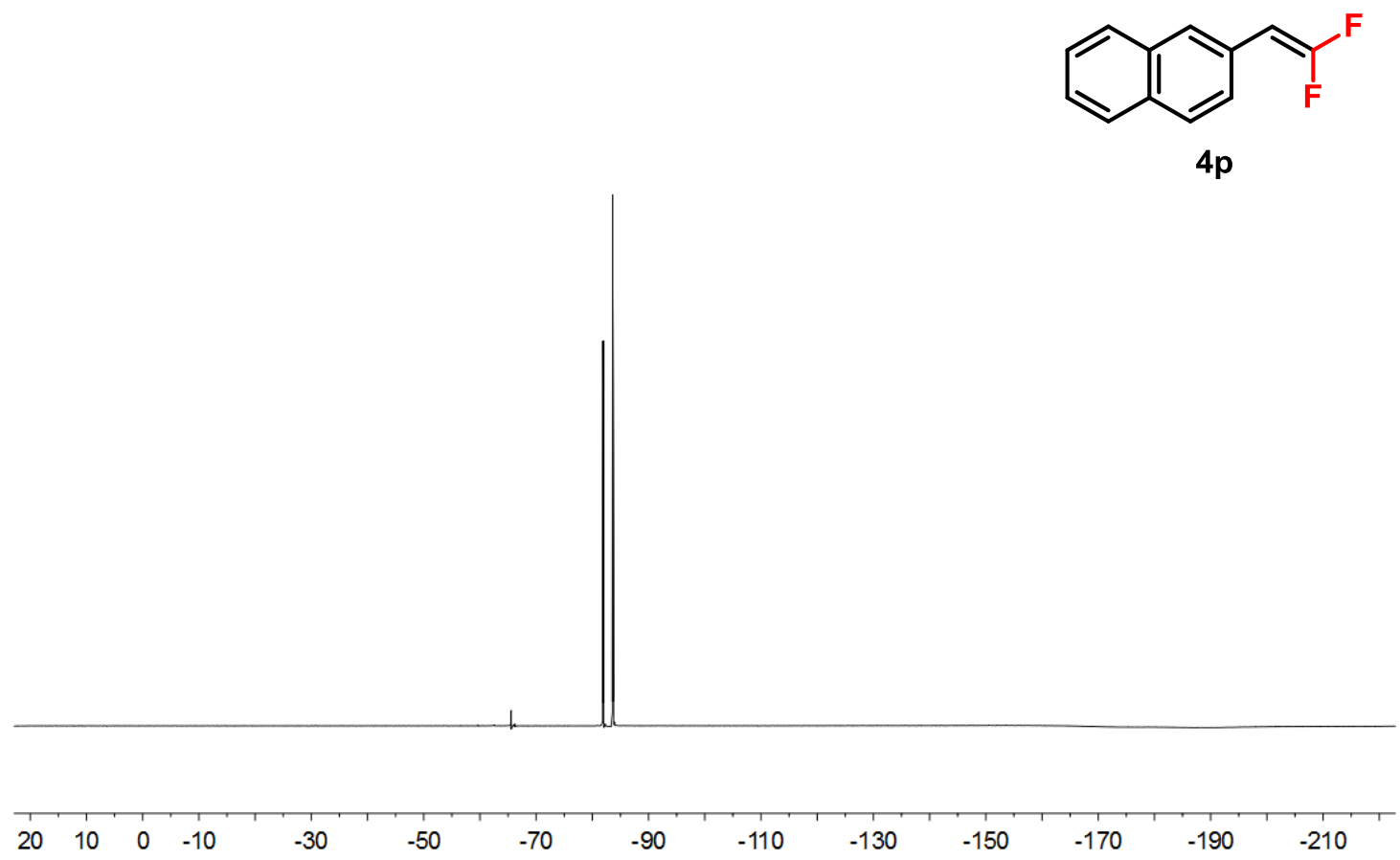

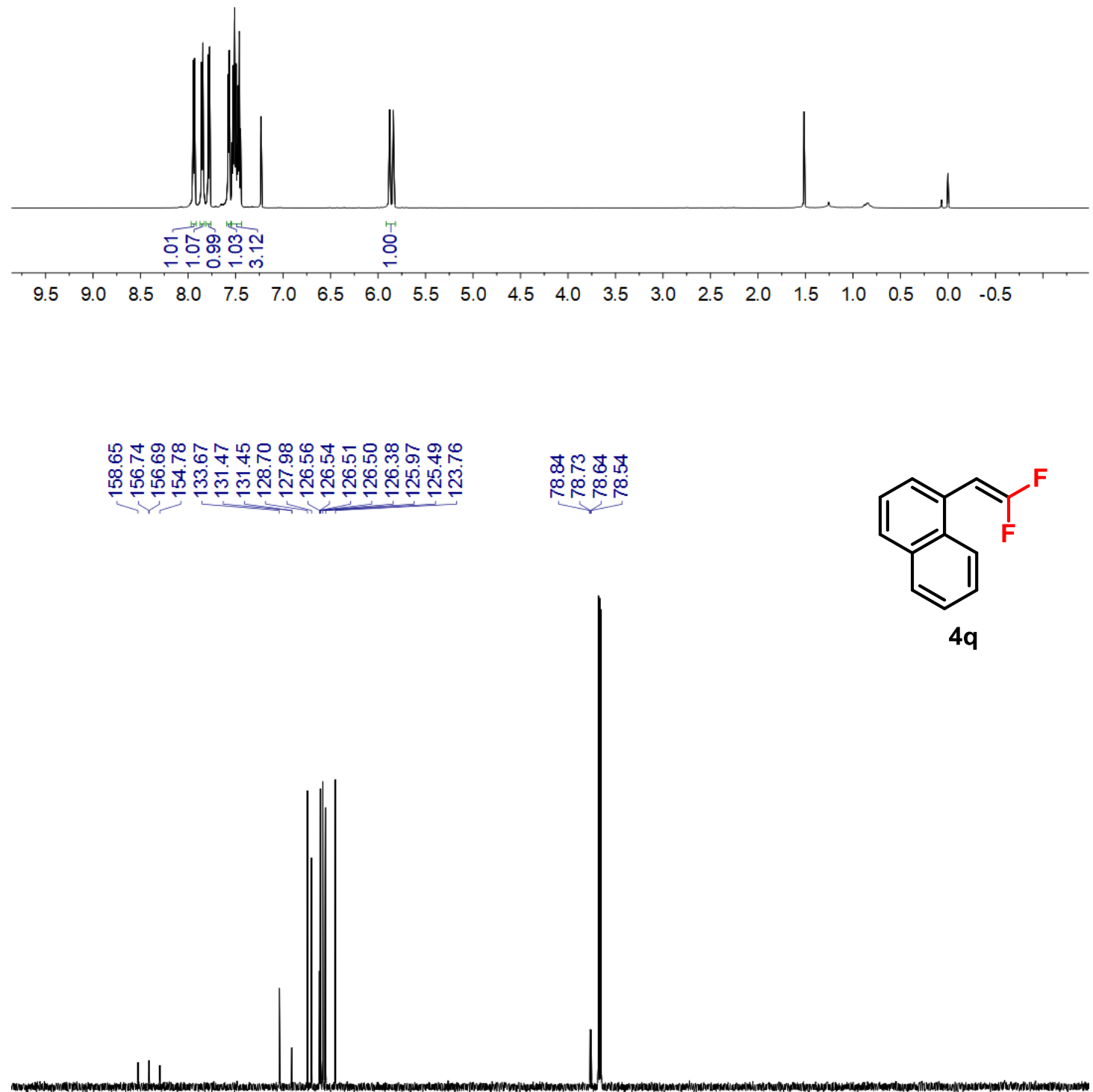

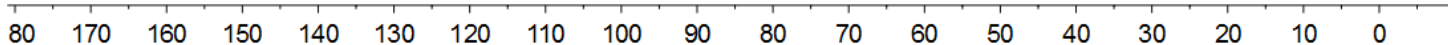


ஸุ

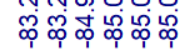
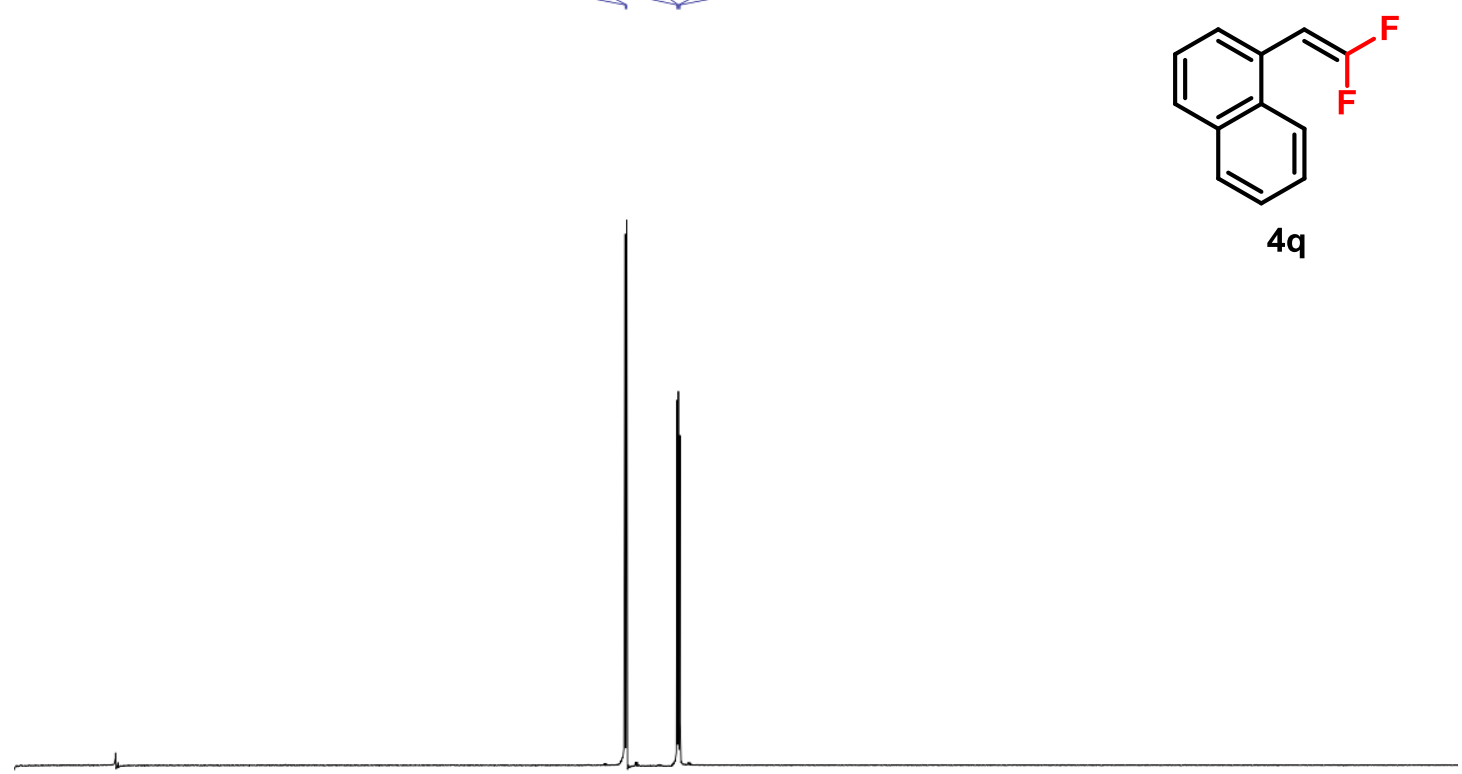

$\begin{array}{llllllllllllllllllllllll}-64 & -66 & -68 & -70 & -72 & -74 & -76 & -78 & -80 & -82 & -84 & -86 & -88 & -90 & -92 & -94 & -96 & -98 & -100 & -102 & -104 & -106 & -108 & -110\end{array}$

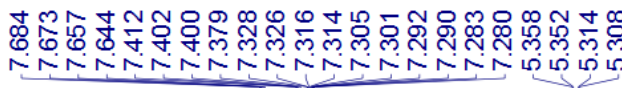

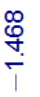
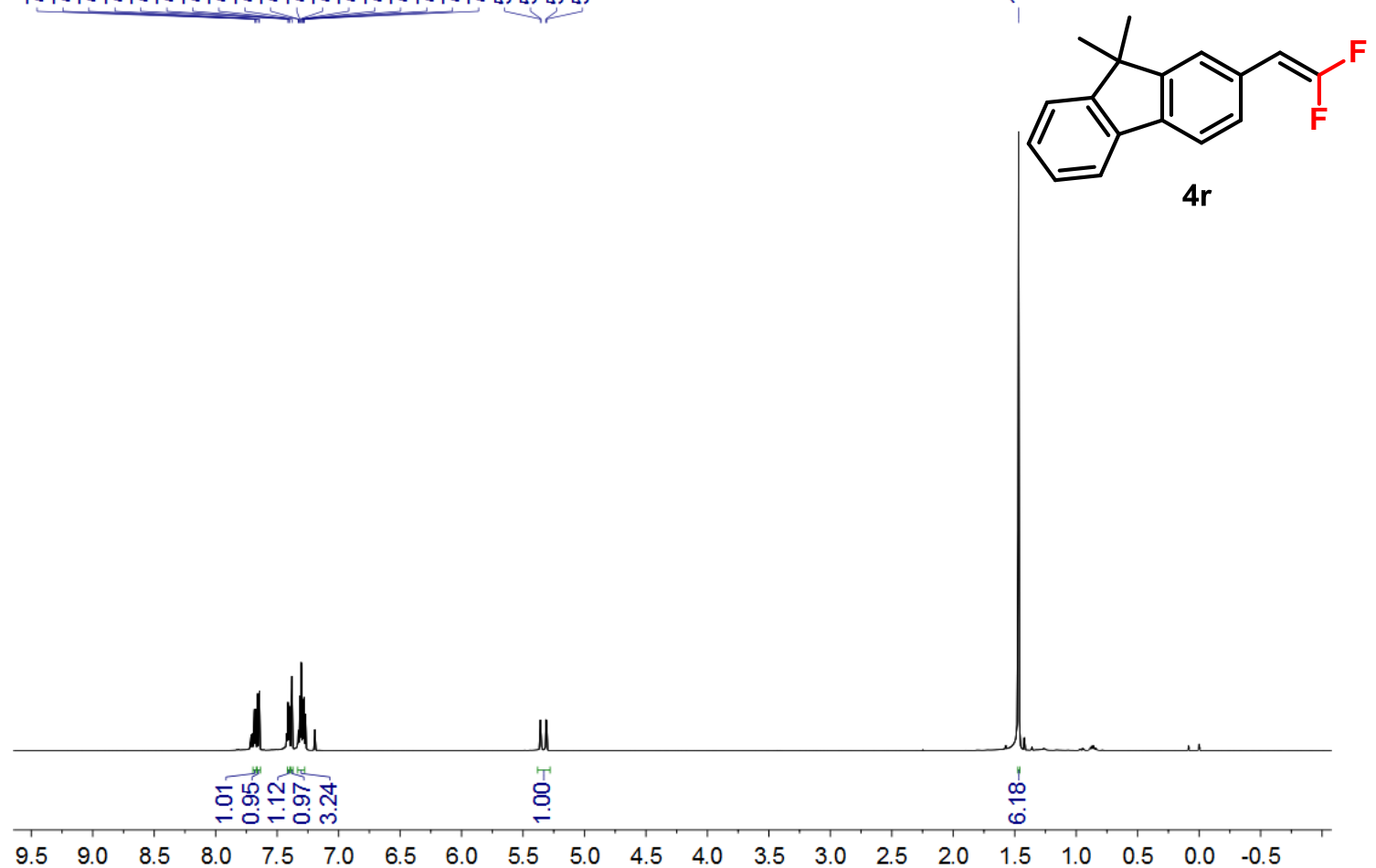

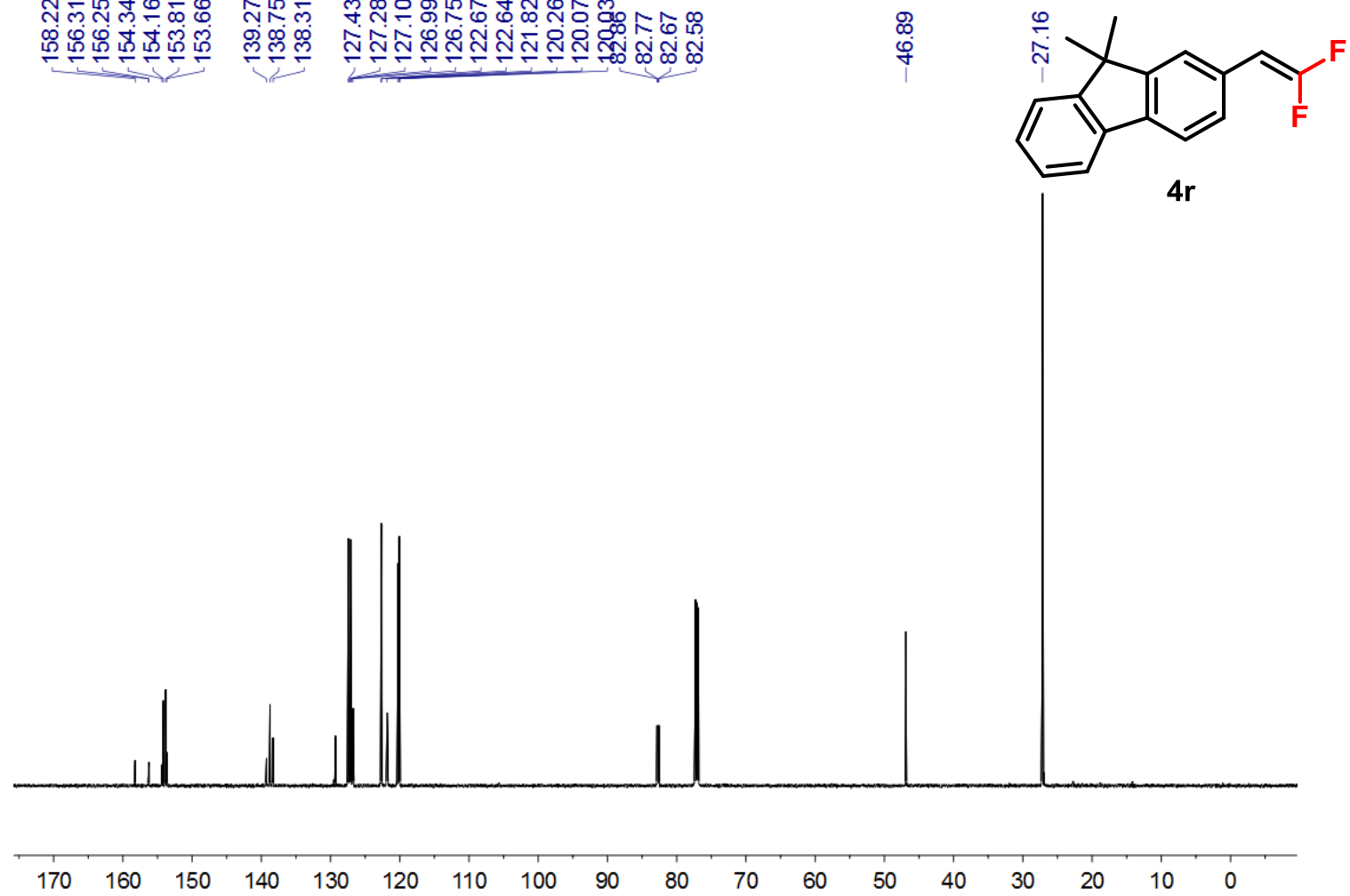

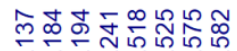

एक्ण

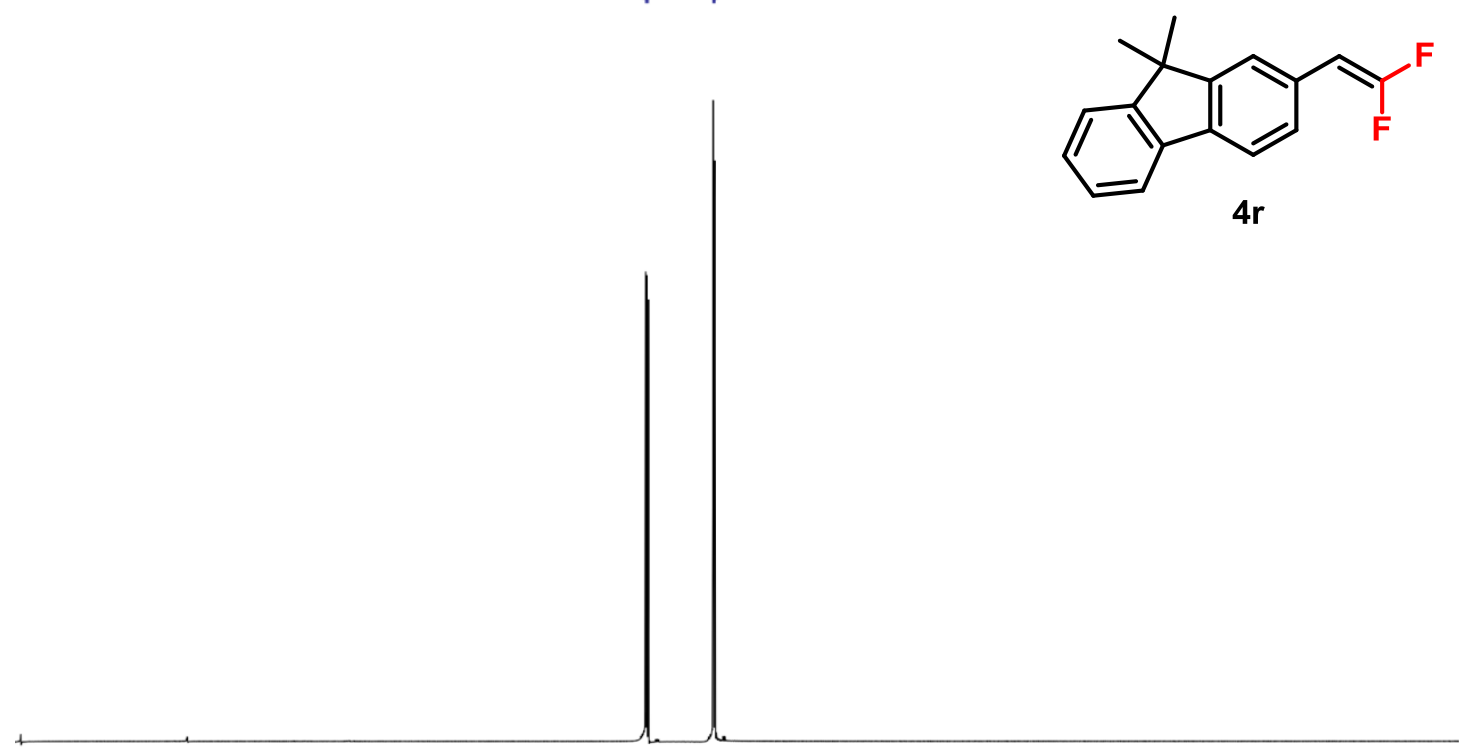

\begin{tabular}{|c|c|c|c|c|c|c|c|c|c|c|}
\hline-62 & -66 & -70 & -74 & -78 & -82 & -86 & -90 & -94 & -98 & -102 \\
\hline
\end{tabular}



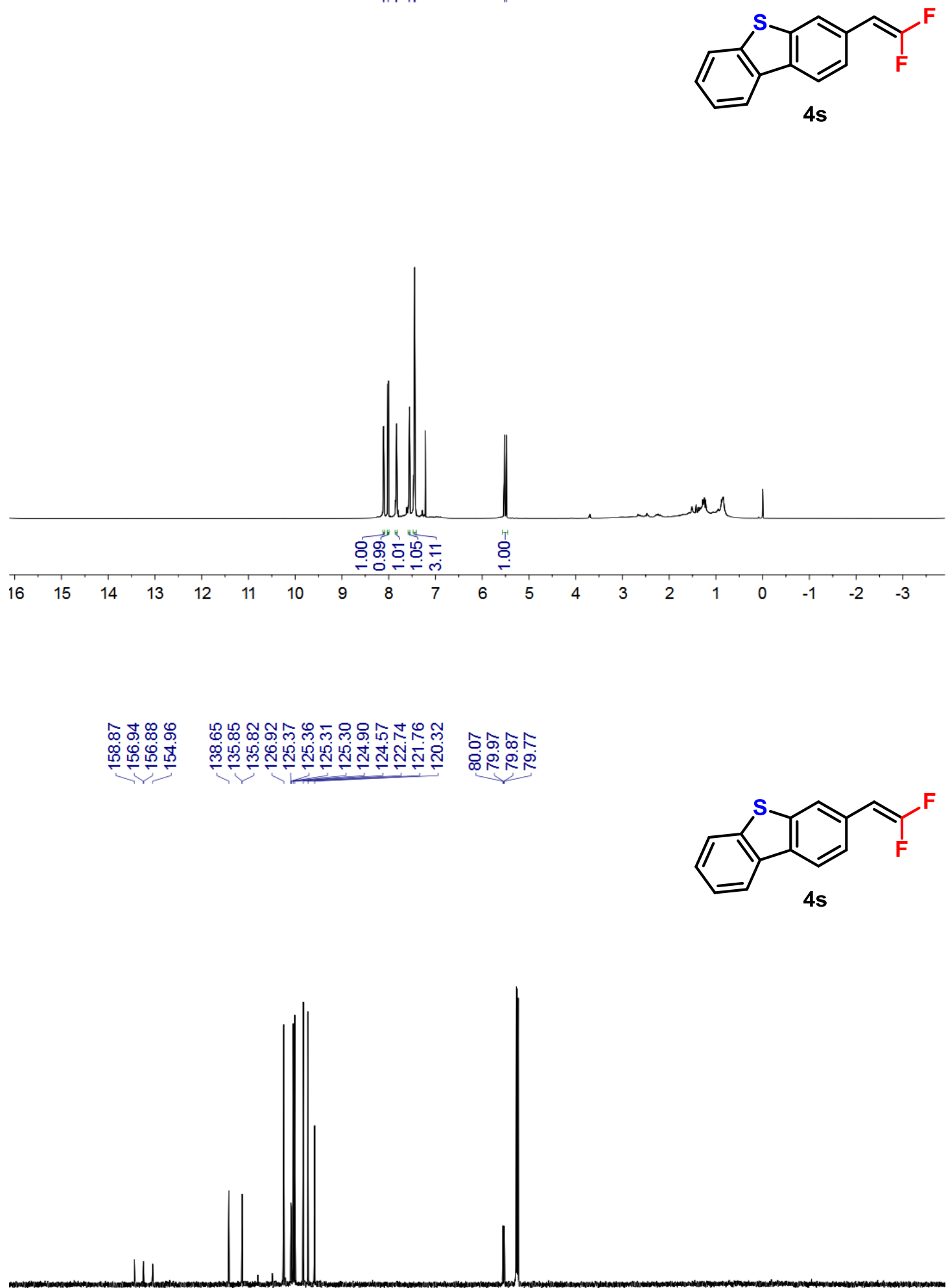

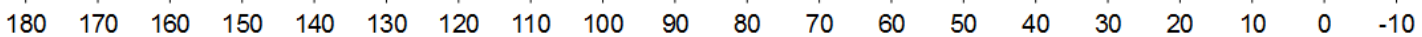




\section{மூ.}

$\infty \infty_{1}^{\infty} \infty \bar{\infty} \infty \bar{\infty}$
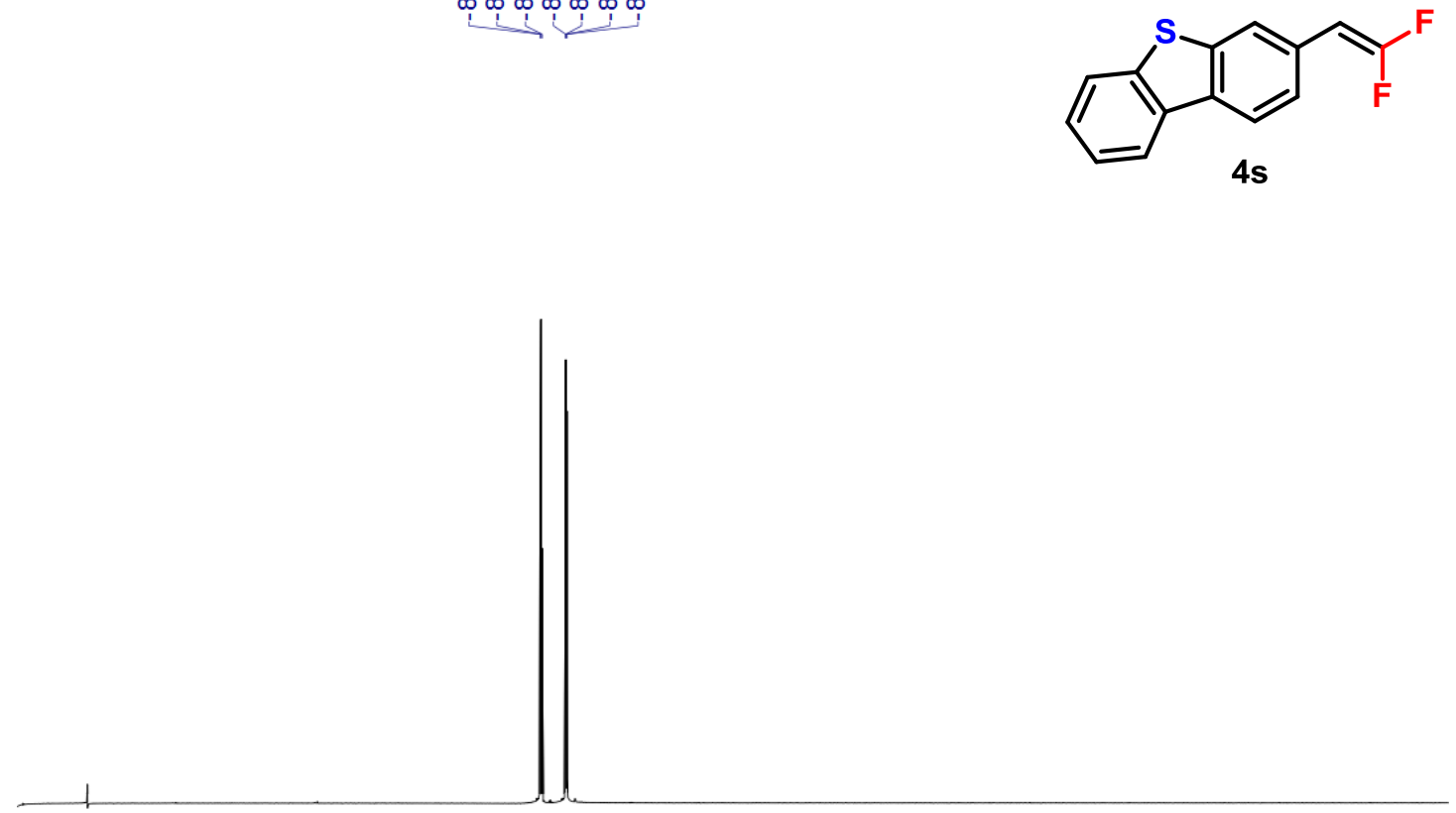

\begin{tabular}{llllllllllllll}
\hline-62 & -66 & -70 & -74 & -78 & -82 & -86 & -90 & -94 & -98 & -104 & -110 & -114
\end{tabular}

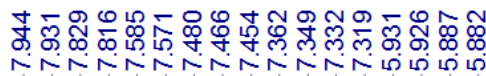
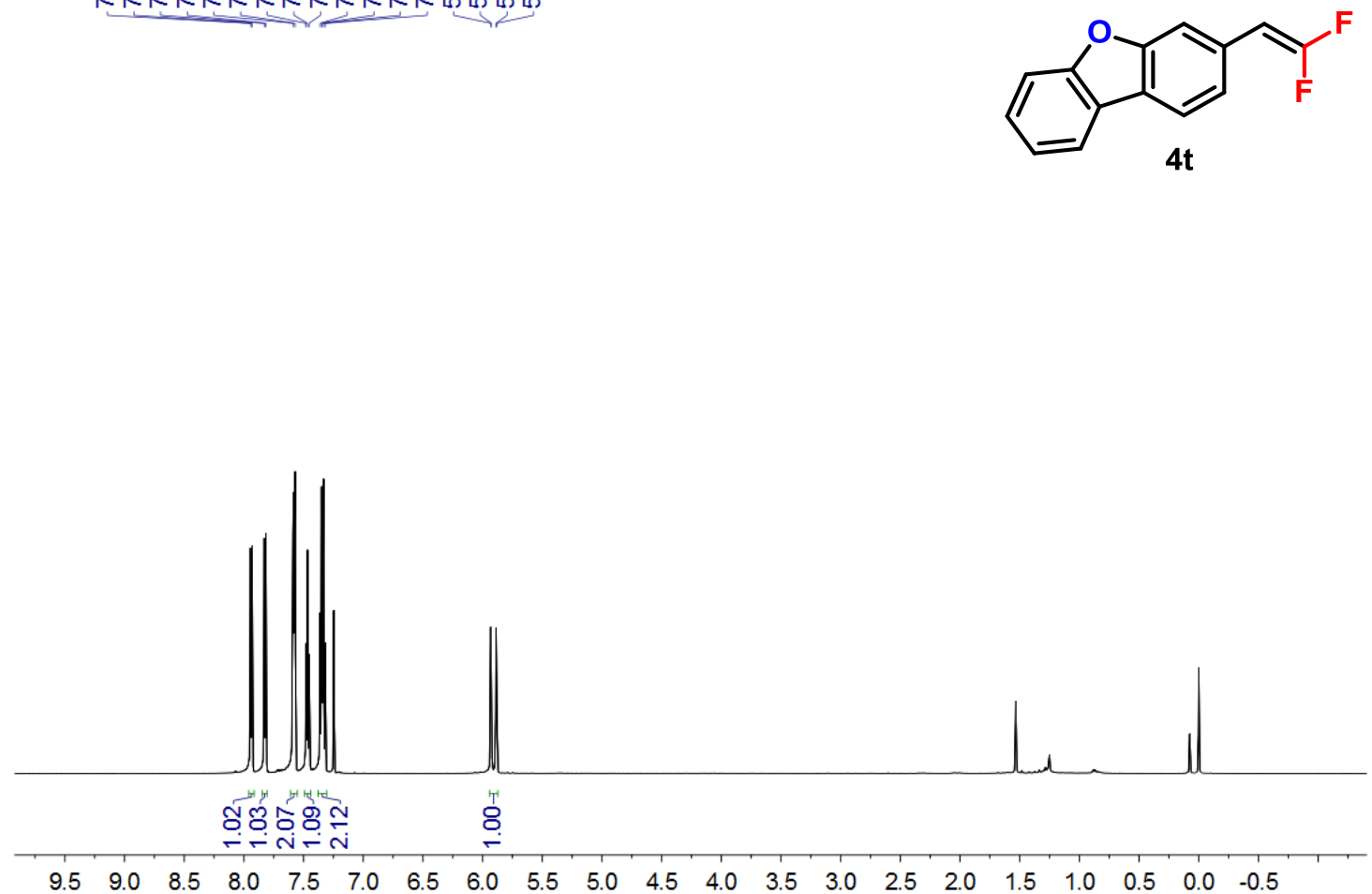


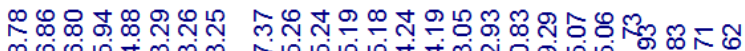

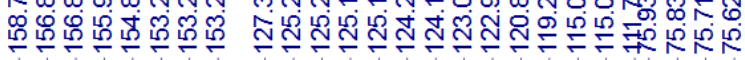
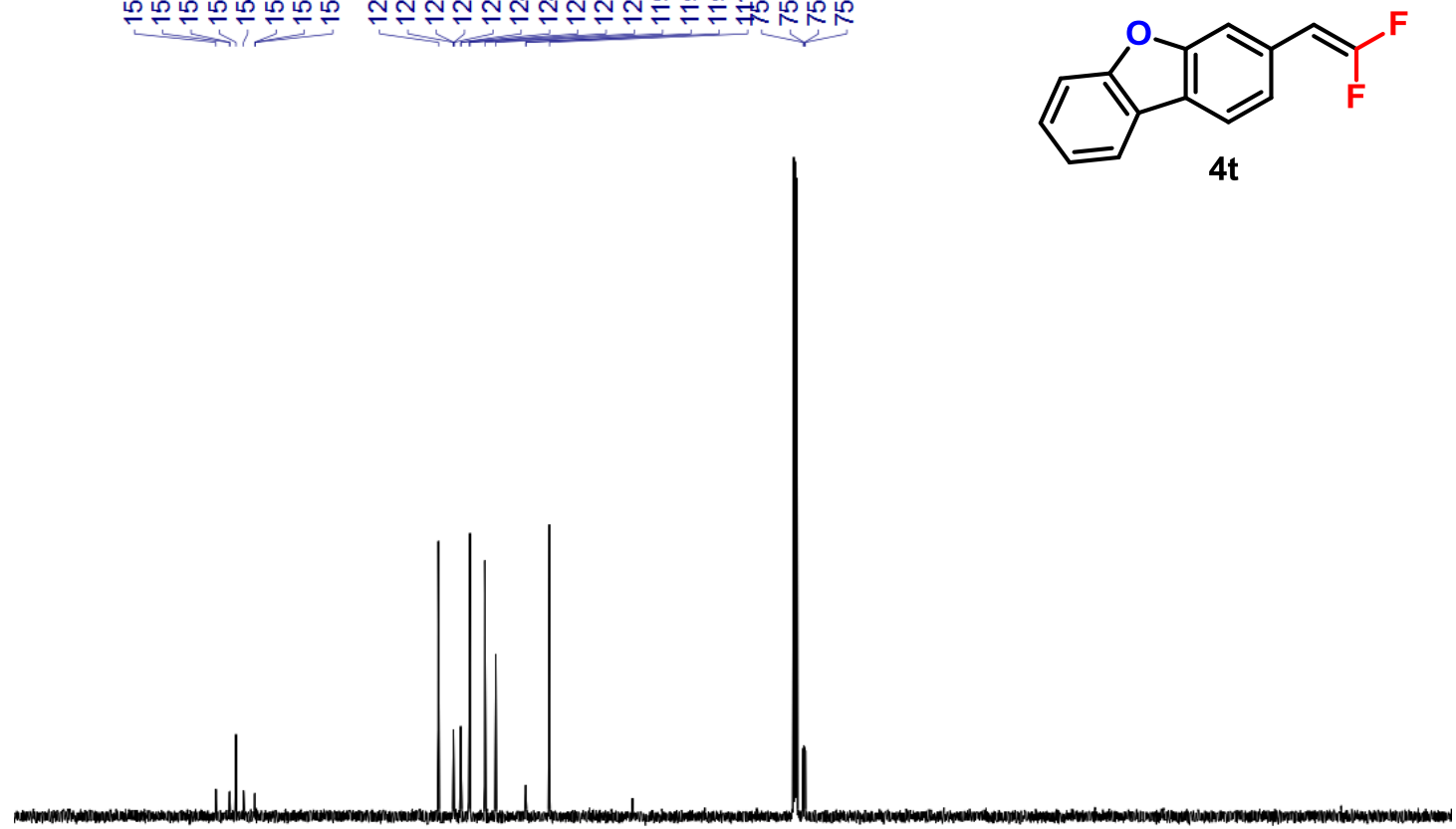

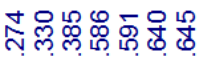

क्ष
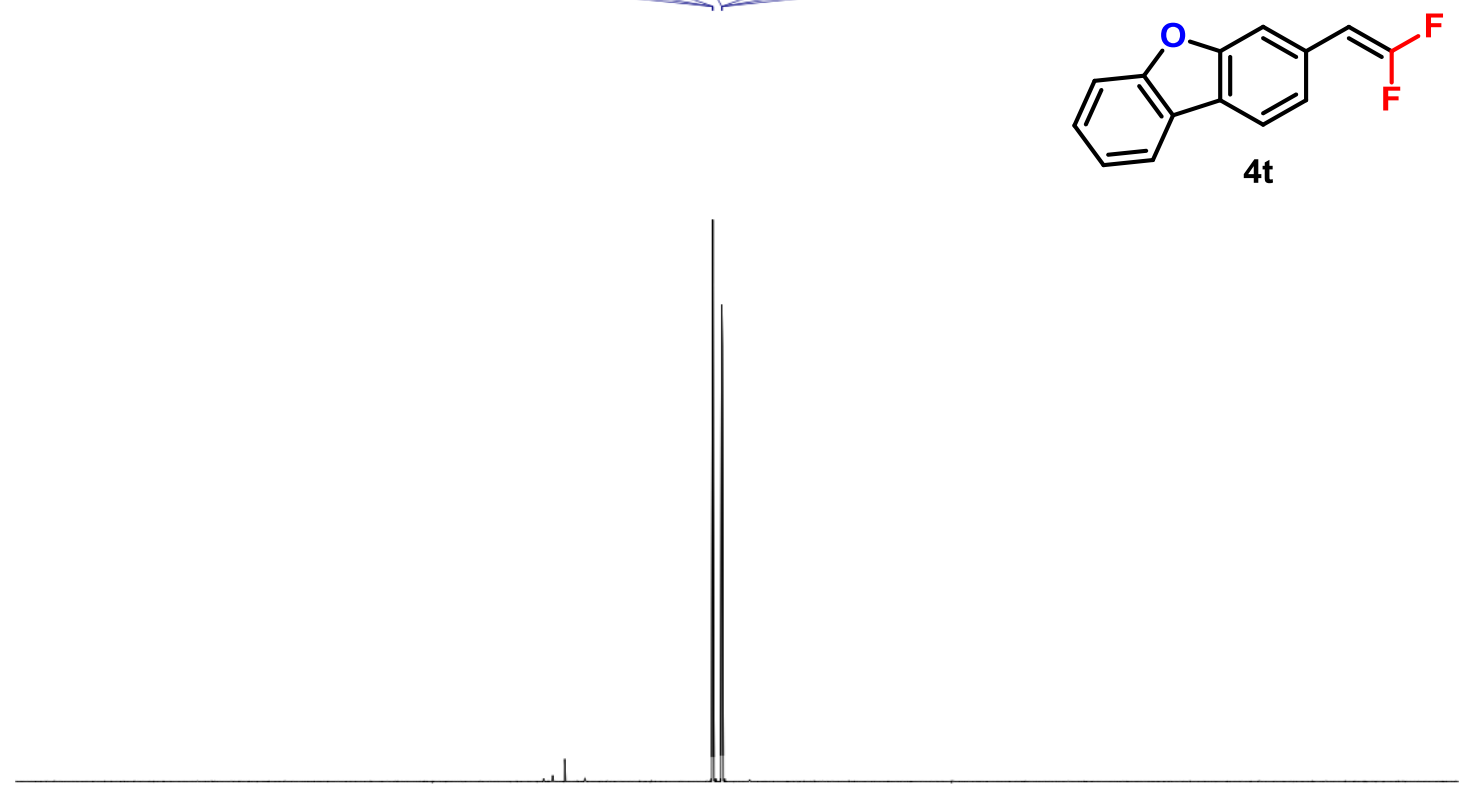

$\begin{array}{llllllllll}10 & 0 & -10 & -20 & -30 & -40 & -50 & -60 & -70 & -80\end{array}$

$-110$

$-130$

$-150$

$-170$ 


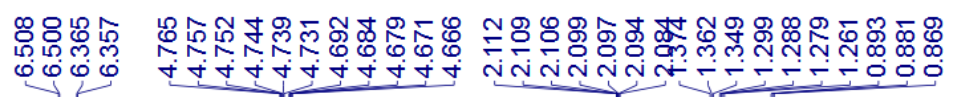

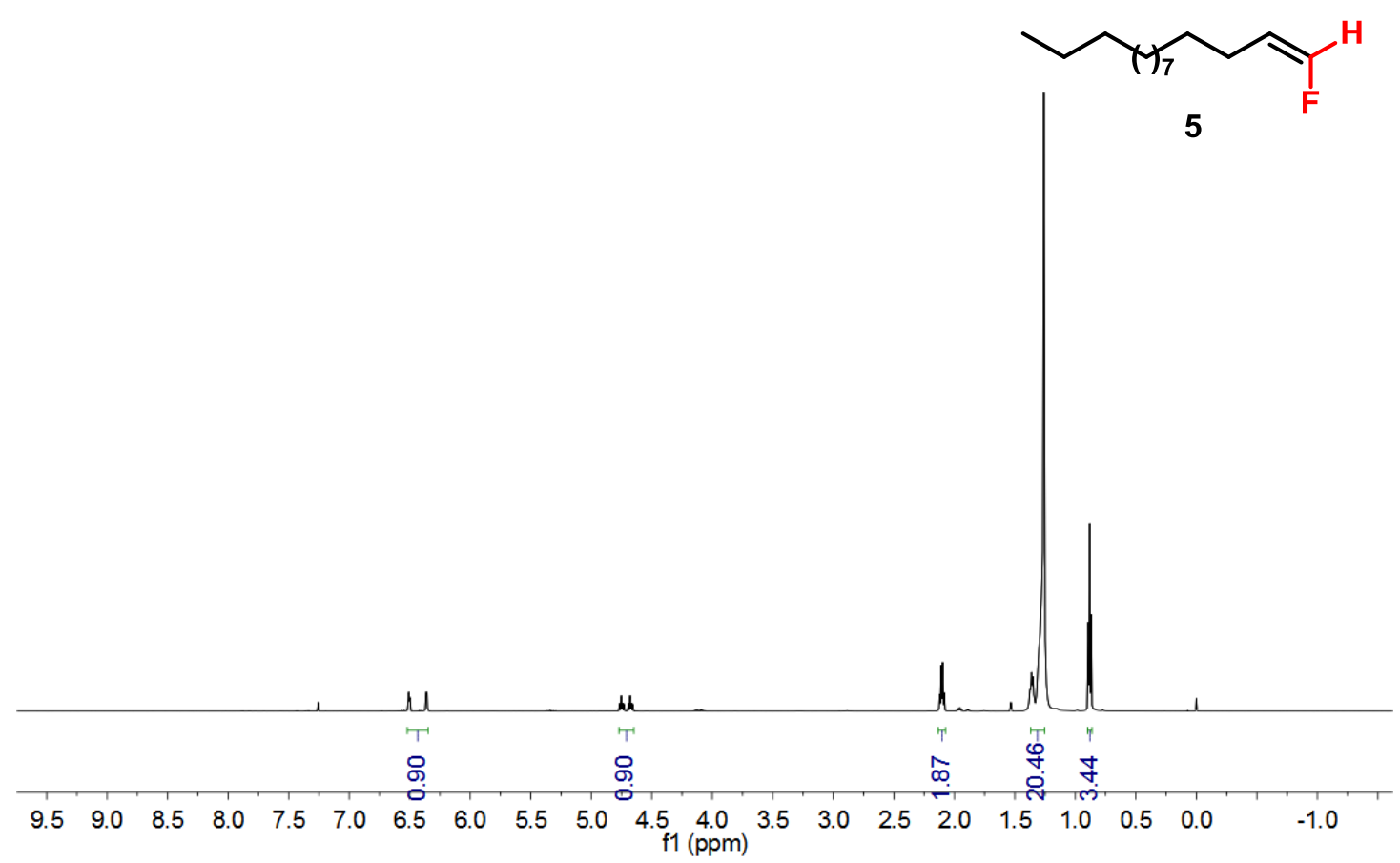

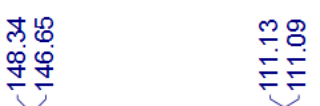

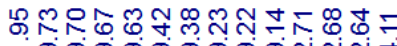

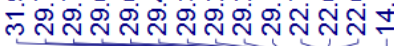
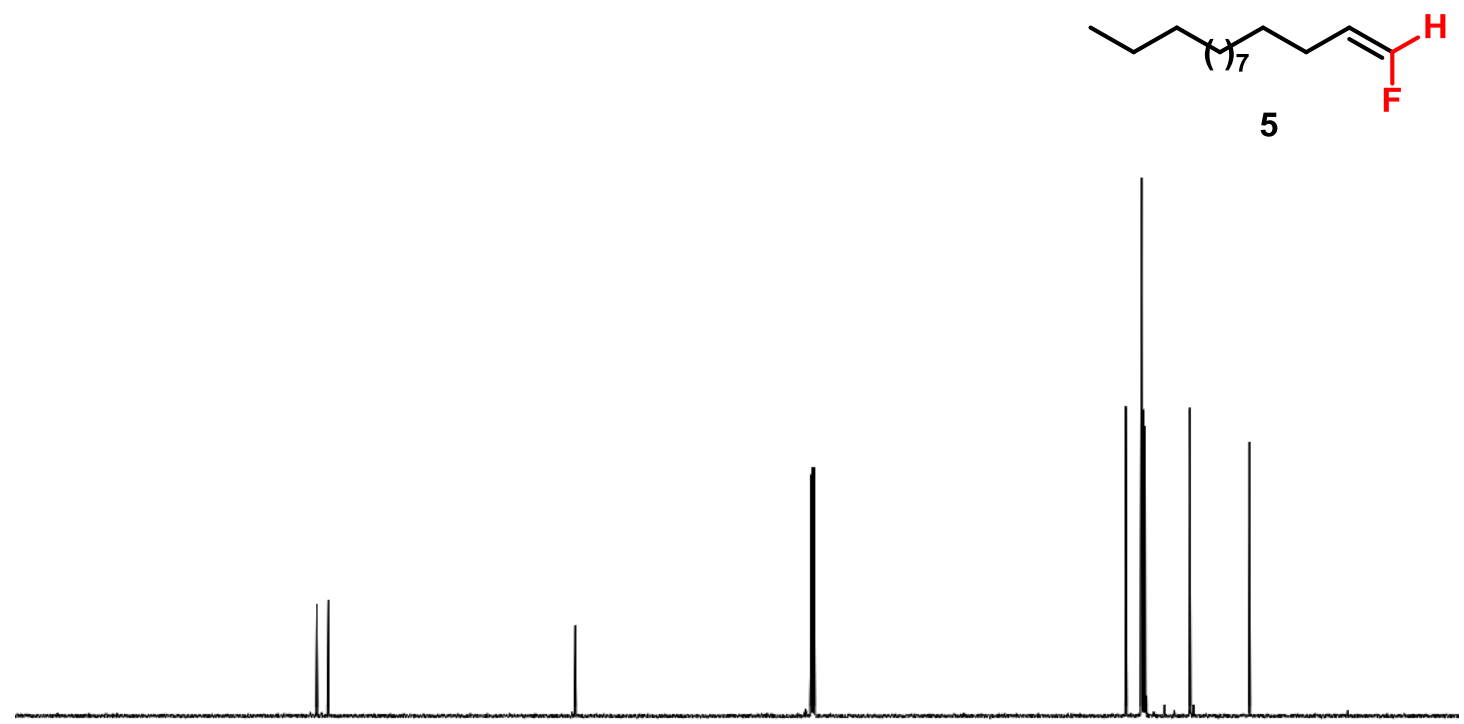

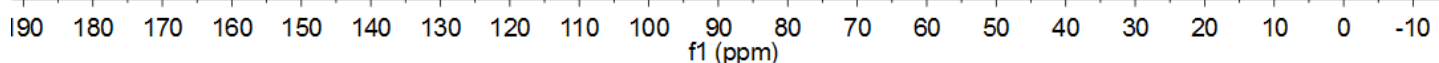


5

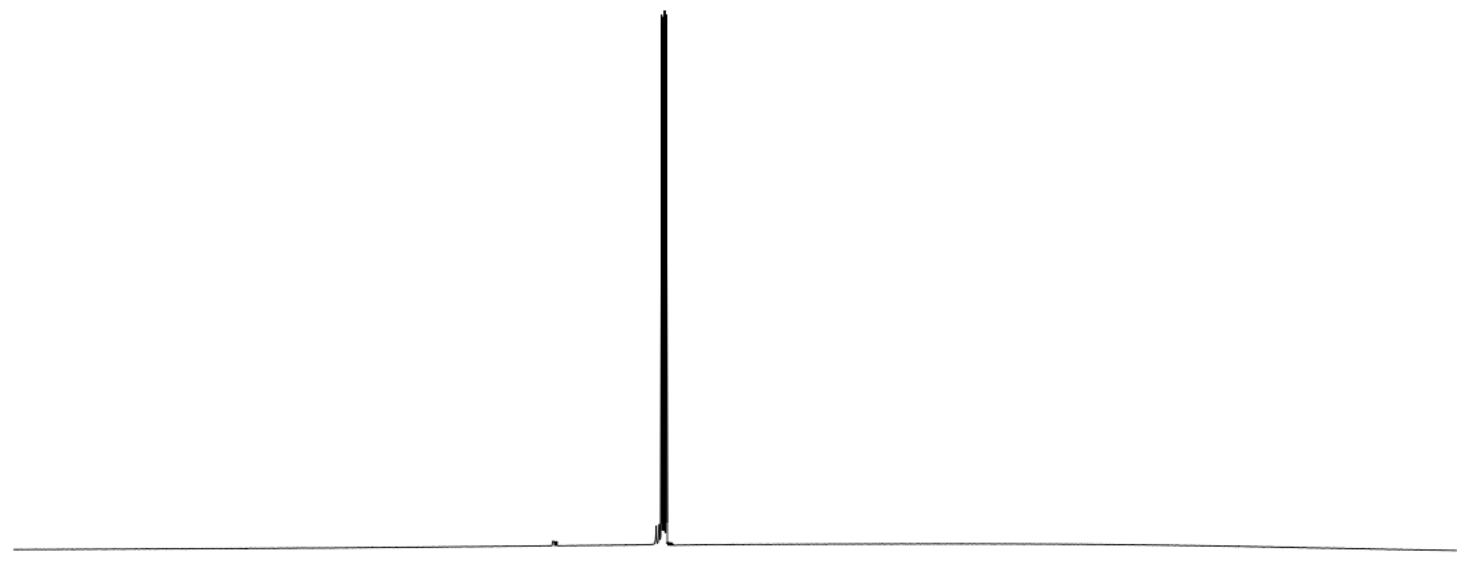

$\begin{array}{lllllllllllllllll}-95 & -100 & -105 & -110 & -115 & -120 & -125 & -130 & -135 & -140 & -145 & -150 & -155 & -160 & -165 & -170 & -175\end{array}$

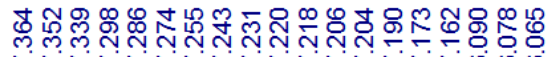

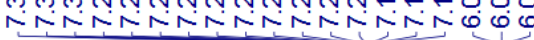

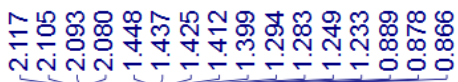
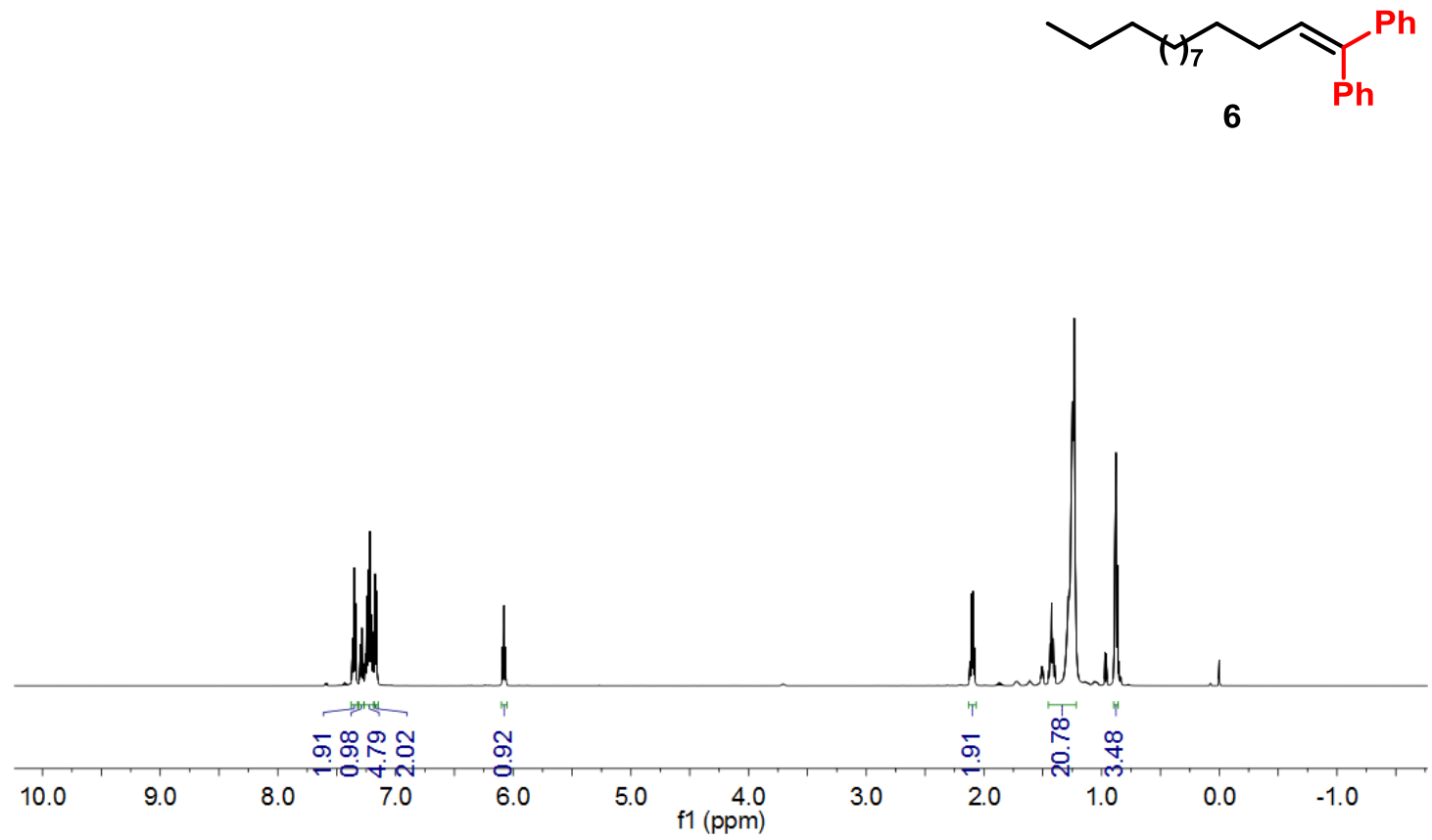

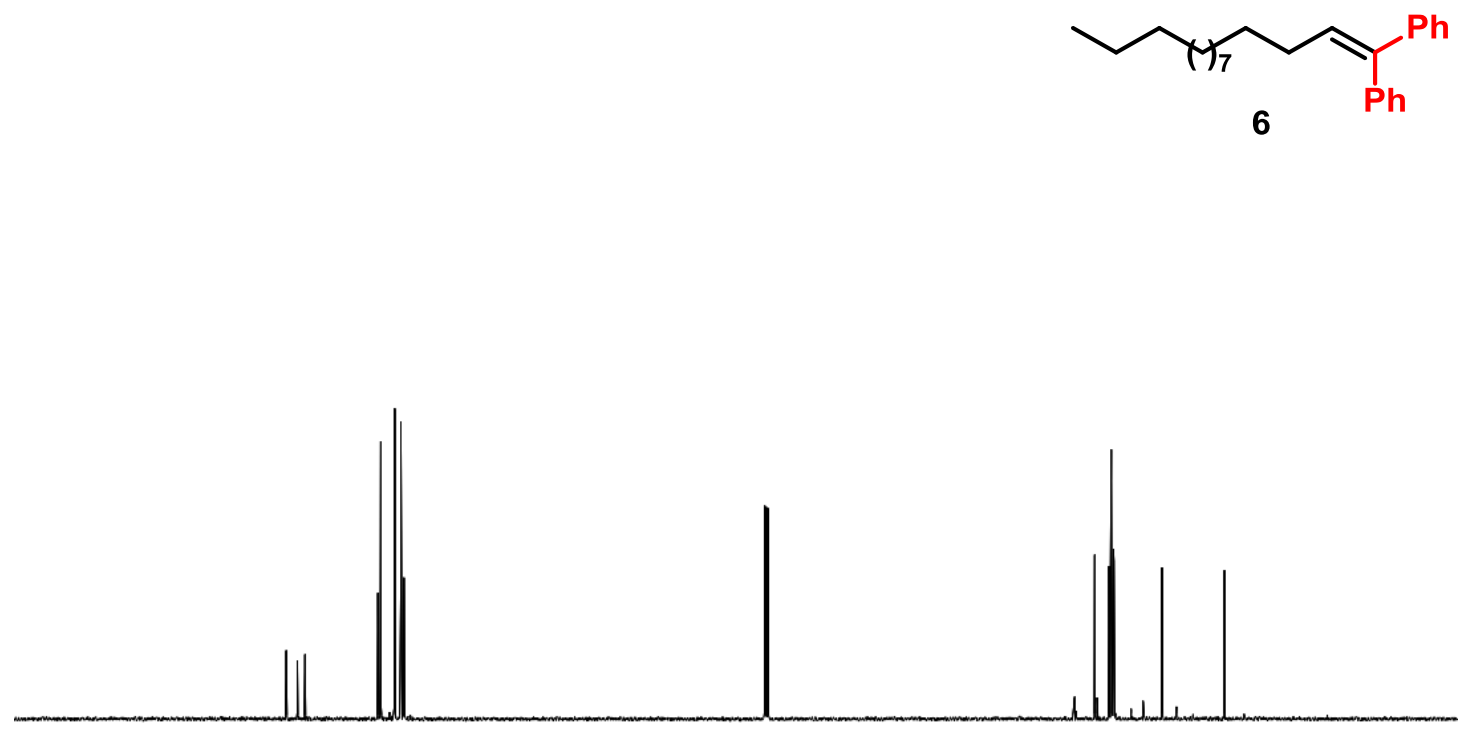

$\begin{array}{llllllllllllllllllll}30 & 170 & 160 & 150 & 140 & 130 & 120 & 110 & 100 & 90 & \begin{array}{c}80 \\ \mathrm{f} 1(\mathrm{ppm})\end{array} & 70 & 60 & 50 & 40 & 30 & 20 & 10 & 0 & -10\end{array}$

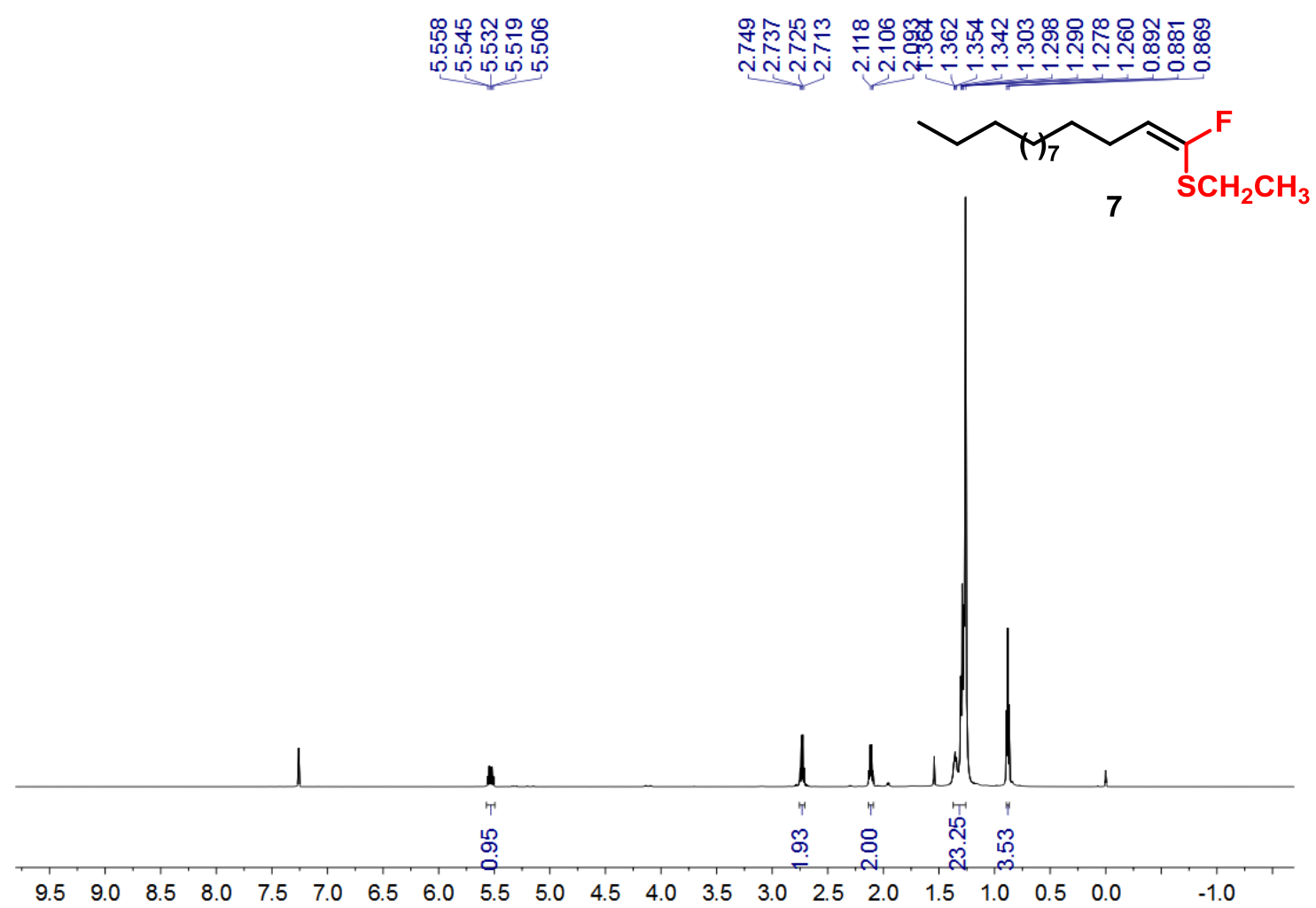



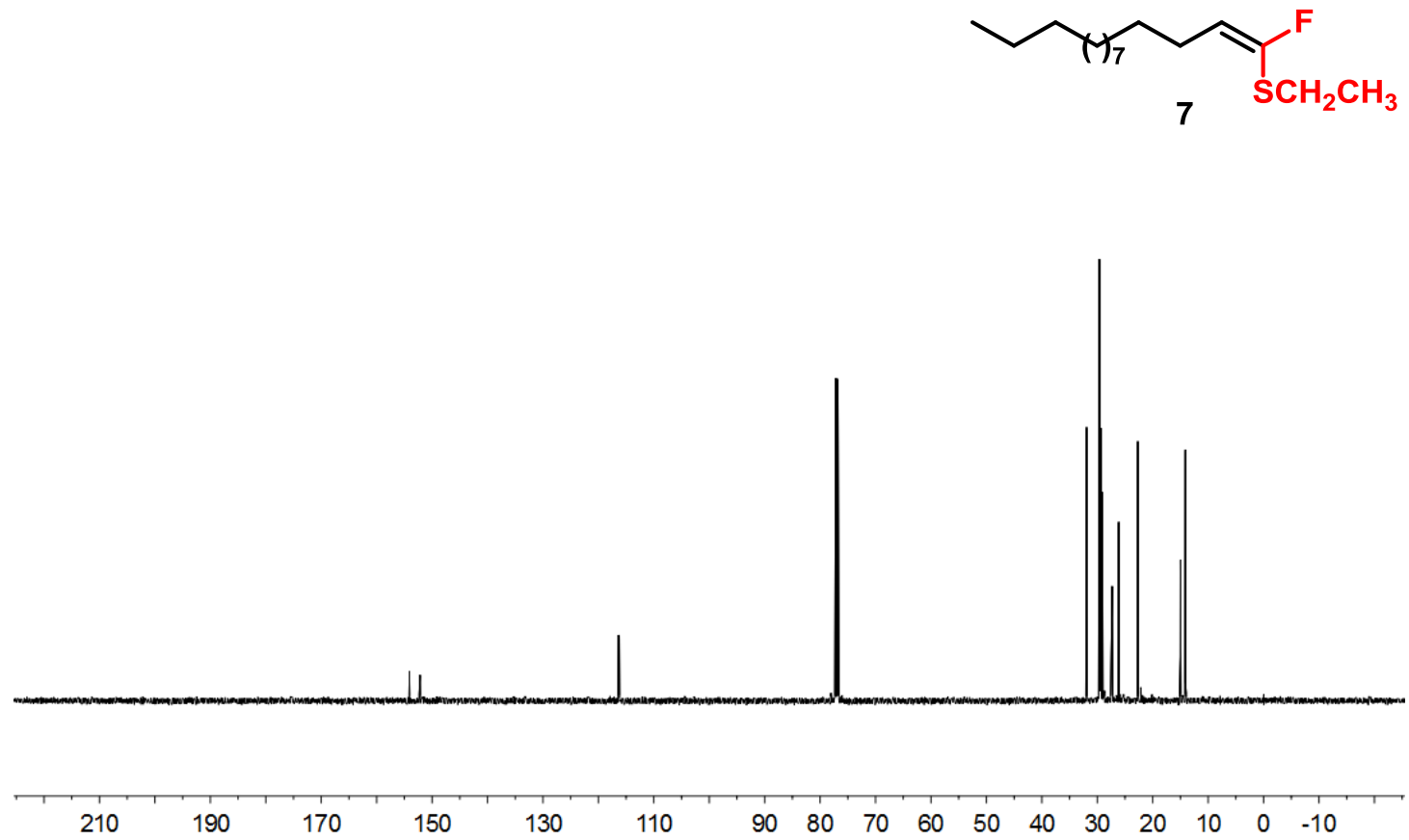

\section{$\infty$
$\infty$
$\infty$
$\infty$
$\infty$
$\infty$
$\infty$
$\infty$}
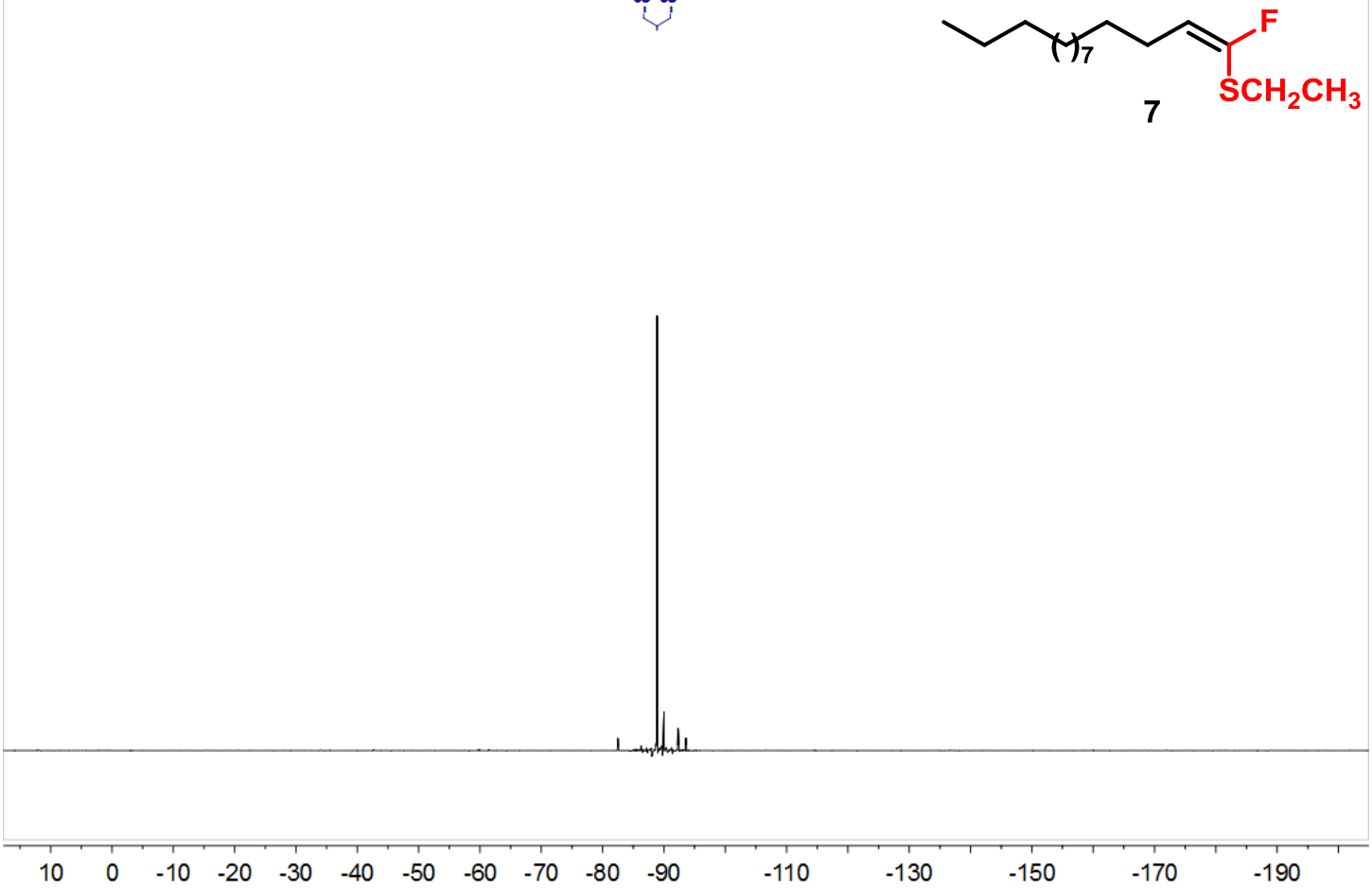


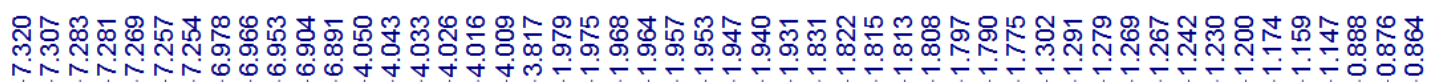
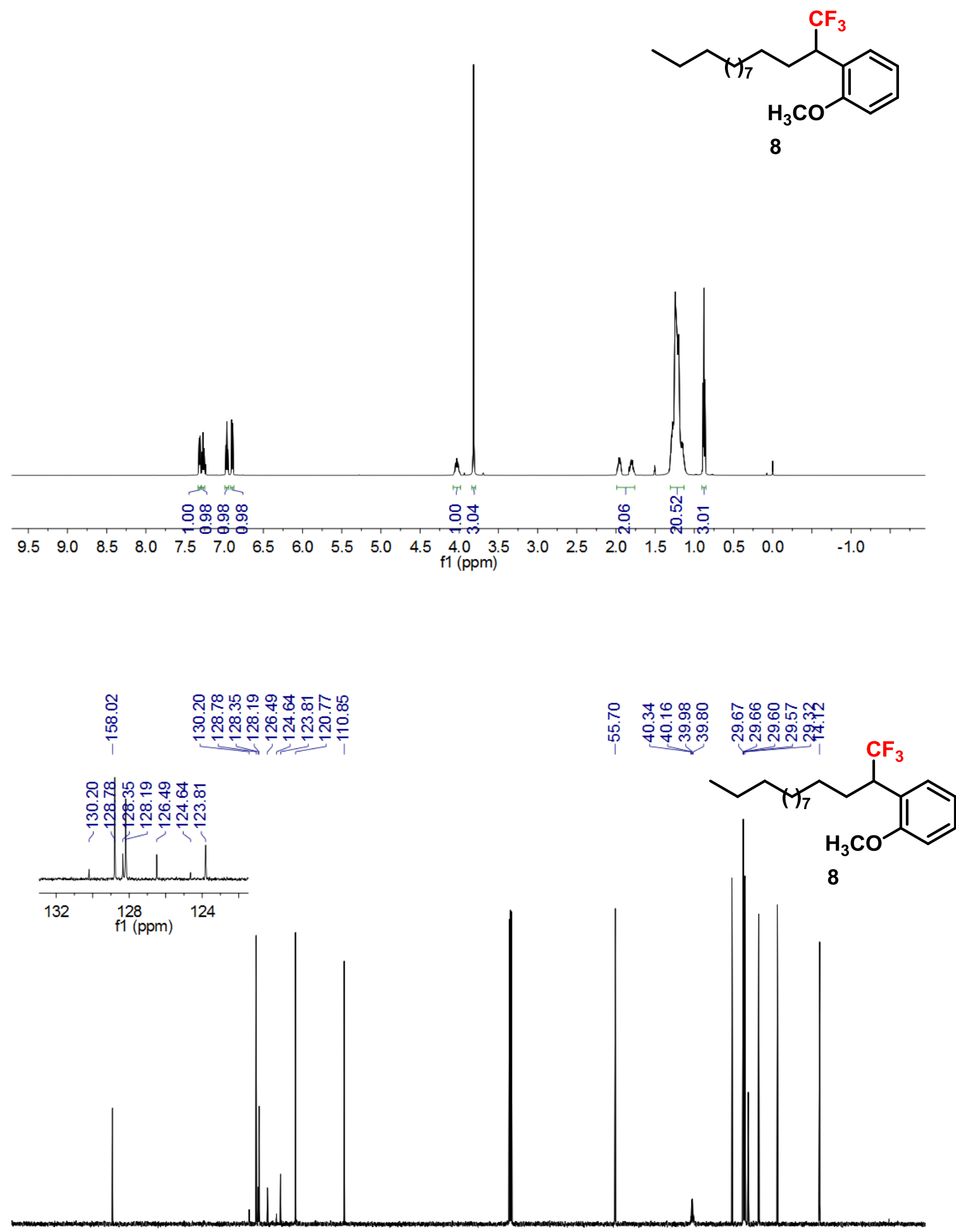

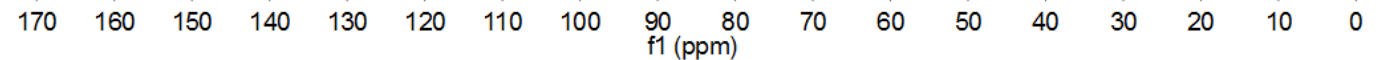




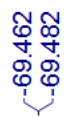

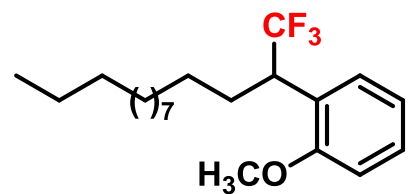

8

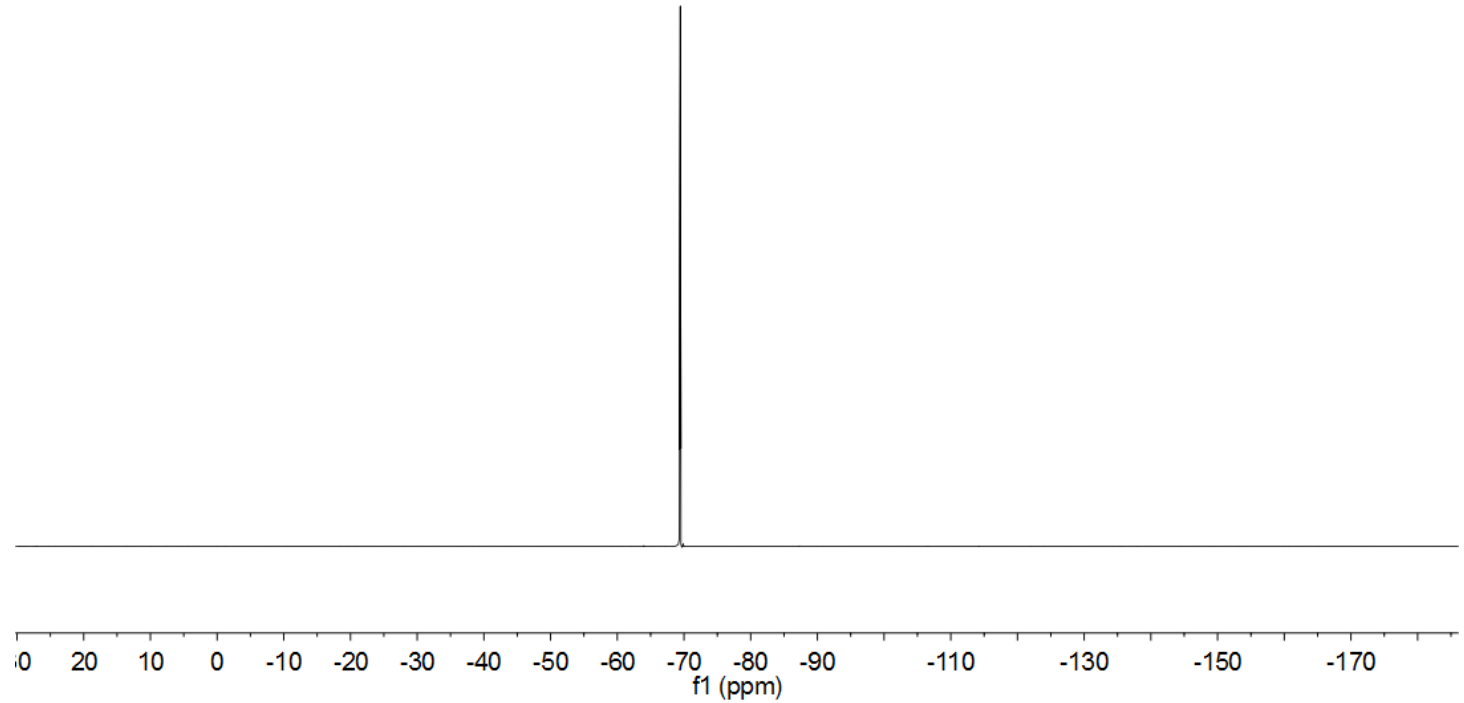

